\title{
Fracture Toughness Data for \\ Brittle Materials
}

R. G. Munro, S. W. Freiman, and T. L. Baker Ceramics Division

Materials Science and Engineering Laboratory

\section{United States Department of Commerce} National Institute of Standards and Technology Gaithersburg, Maryland 20899 



\section{Fracture Toughness Data for \\ Brittle Materials}

R. G. Munro, S. W. Freiman, and T. L. Baker

Ceramics Division

Materials Science and Engineering Laboratory

National Institute of Standards and Technology

Gaithersburg, Maryland 20899

April 1998

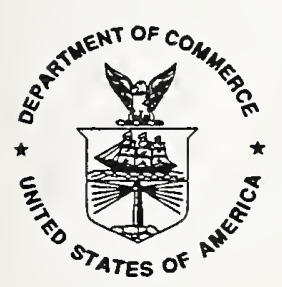

U. S. Department of Commerce

William M. Daley, Secretary

Technology Administration

Gary R. Bachula, Acting Under Secretary for Technology

National Institute of Standards and Technology

Raymond G. Kammer, Director 
Certain commercial equipment, instruments, or materials are identified in this document to specify adequately the experimental procedures and conditions. Such identification does not imply recommendation or endorsement by the National Institute of Standards and Technology, nor does it imply that the materials or equipment identified are necessarily the best available for the purpose.

National Institute of Standards and Technology NISTIR 6153

Natl. Inst. Stand. Technol.

NISTIR 6153

153 printed pages (April 1998) 


\title{
Fracture Toughness Data for Brittle Materials
}

R. G. Munro, S. W. Freiman, and T. L. Baker

Ceramics Division, National Institute of Standards and Technology

Gaithersburg, MD 20899

\begin{abstract}
Fracture toughness data, as represented by the critical stress intensity factor, $K_{\mathrm{Ic}}$, and the fracture energy, $\gamma$, have been compiled from publicly accessible sources for a wide range of brittle materials with an emphasis on structural ceramics and closely related materials. The results are organized according to the material designation and are presented in annotated tables.
\end{abstract}

Key Words

Brittle materials; ceramics; data; database; fracture toughness; structural ceramics

\section{Table of Contents}

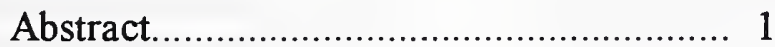

Table of Contents....................................... 1

1. Introduction............................................ 2

2. Organization of the Data........................ 4

3. Notation and Conventions....................... 4

3.1 Measurement Methods...................... 4

3.2 Property Symbols................................ 7

3.3 Units............................................. 7

3.4 Uncertainties................................... 7

4. Index of Materials ................................... 8

5. Tables of Data....................................... 11 


\section{Introduction}

Brittle materials are subject to rapid crack extension upon the application of tensile stresses. The capacity of a material to resist the extension of a crack is called fracture toughness. ${ }^{1,2}$

Analytical descriptions of fracture behavior are commonly based on linear stress analysis. In this approach, the focus is on the stress in the vicinity of a crack tip where the curved boundaries lead to an enhanced stress state. A parameter, $K$, called the stress intensity factor, is introduced as a measure of the enhanced value of the stress. The symbol $K_{\mathrm{I}}$, called the Mode I stress intensity factor, is used for the particular case of tensile crack extension. The critical value of the stress intensity factor, $K_{\mathrm{Ic}}$, commonly called the fracture toughness, is defined as the value at which bond rupture occurs at the crack tip. Linear stress analysis for tensile cracks leads to the relation

$$
K_{I c}^{2}=\Gamma E^{\prime}=2 \gamma E^{\prime}
$$

where $E^{\prime}=E$ for plane stress and $E^{\prime}=E /\left(1-v^{2}\right)$ for plane strain; $E$ is the elastic modulus; $v$ is Poisson's ratio; $\Gamma$ is the mechanical energy release rate for fracture; and $2 c_{0}$ is the initial crack size. The quantity, $\gamma=\Gamma / 2$, is often called the fracture energy and is reported more commonly than $\Gamma$. Values of $K_{\mathrm{Ic}}$ and $\gamma$ are given in the present compiliation.

Several procedures for estimating fracture toughness involve measuring the fracture strength, $\sigma_{f}$ of a cracked specimen and then evaluating the fracture toughness in the form

$$
K_{I c}=Y \sigma_{f} c_{0}^{1 / 2}
$$

where $Y$ is a dimensionless numeric factor that depends on the test configuration and the shape of the initial crack. ${ }^{3}$ Numerous experimental designs have been developed in attempts to minimize the uncertainties in the value of $K_{\mathrm{Ic}}$ arising from the geometric uncertainties of the test configuration and the initial crack.

The present report presents a compilation of fracture toughness $\left(K_{\mathrm{Ic}}\right)$ and fracture energy $(\gamma)$ values for a wide range of brittle materials determined by a variety of test methods. The results are obtained primarily from publicly accessible published sources; in all cases, the sources of the data and the methods used in the measurements are cited explicitly. The measurement methods are described in Section 3.1.

Overviews of the data are provided by Fig. 1, which shows a distribution of all the fracture toughness values reported in the compilation, and Fig. 2, which shows a distribution of the fracture energy values. It can be seen that most of the toughness values are less than $8 \mathrm{MPa} \cdot \mathrm{m}^{1 / 2}$. The smallest reported value is $0.16 \mathrm{MPa} \cdot \mathrm{m}^{1 / 2}$ for cracks parallel to a cleavage plane of a sodium $\beta$-alumina single crystal. ${ }^{4}$ Normal to that plane, the toughness for this crystal is 2.0 $\mathrm{MPa} \cdot \mathrm{m}^{1 / 2}$. The unusual toughness values greater than $10 \mathrm{MPa} \cdot \mathrm{m}^{1 / 2}$ were reported for various stabilized tetragonal zirconia polycrystals. ${ }^{5,6}$ 


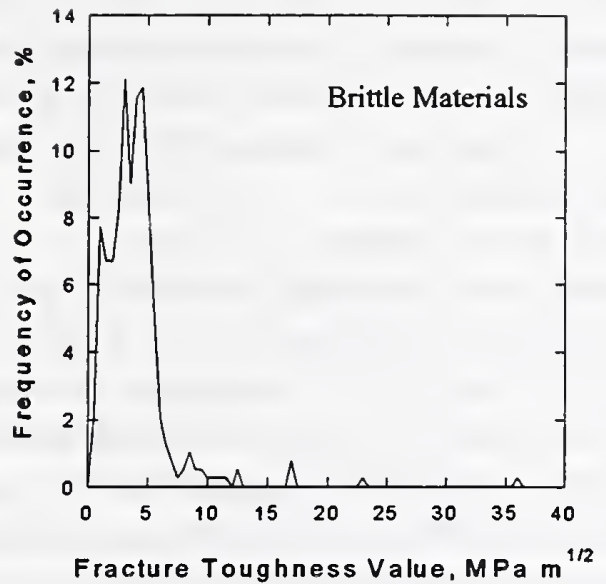

Figure 1: Distribution of Fracture Toughness Values

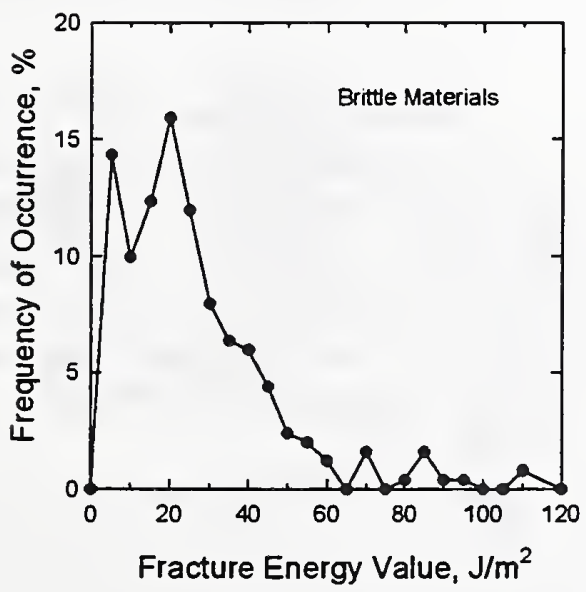

Figure 2: Distribution of Fracture Energy Values
References for the Introduction

1. A. A. Griffith, "Phenomena of Rupture and Flow in Solids," Philosophical Transactions of the Royal Society of London, Vol. 221, pp. 163-198 (1921).

2. G. R. Irwin and P. C. Paris, "Fundamental Aspects of Crack Growth and Fracture" in Fracture, An Advanced Treatise, Vol. III, edited by H. Liebowitz, Academic Press, New York (1971), pp. 1-46.

3. J. B. Wachtman, Mechanical Properties of Ceramics, John Wiley \& Sons, Inc., New York (1996).

4. D. C. Hitchcock and L. C. De Jonghe, "Fracture Toughness Anistropy of Sodium Beta-Alumina," Journal of the American Ceramic Society, Vol. 66, No. 11, pp. C-204 C-205 (1983).

5. J. Wang, W.M. Rainforth, T. Wadsworth, and R. Stevens, "The Effects of Notch Width on the SENB Toughness for Oxide Ceramics," Journal of the European Ceramic Society, Vol. 10, pp. 21-31 (1992).

6. K. Tsukuma and M. Shimada, "Strength, Fracture Toughness and Vickers Hardness of $\mathrm{CeO}_{2}$-Stabilized Tetragonal $\mathrm{ZrO}_{2}$ Polycrystals (Ce-TZP)," Journal of Materials Science, Vol. 20, pp. 1178-1184 (1985); and K. Tsukuma, "Mechanical Properties and Thermal Stability of $\mathrm{CeO}_{2}$-Containing Tetragonal Zirconia Polycrystals," American Ceramic Society Bulletin, Vol. 65, pp. 1386-1389 (1986). 


\section{Organization of the Data}

Results are grouped by the material designation (a chemical formula, a common name, or a commercial name). A list of designations is given in section 4. Each material designation is presented separately. Therefore, each table begins with a brief description of the material, starting with the material designation. The references for the data are given in each case after the material is defined. Each table ends with an annotated summary of the numeric data which are presented in a fixed format. In all cases, the column headers clearly define the content.

\section{Notation and Conventions}

A small number of conventions, abbreviations, and special symbols are used in this report so that the space allocated for comments within the data tables can be used efficiently. For example, abbreviations and acronyms are used to indicate the measurement methods used in determining the property values.

\subsection{Measurement Methods}

$\mathrm{AMDCB}=$ Applied Moment Double Cantilever Beam

$\mathrm{CF}=$ Controlled Flaw

$\mathrm{CNB}=$ Chevron Notch Beam

$\mathrm{DCB}=$ Double Cantilever Beam

DT $=$ Double Torsion

$\mathrm{HI}=$ Hertzian Indentation

ICS $=$ Indentation Crack Size

IS = Indentation Strength

NDC $=$ Notched Diametral Compression

$\mathrm{SCF}=$ Surface Crack in Flexure

SENB $=$ Single-Edge Notched-Beam
SEPB $=$ Single-Edge Precracked Beam $\mathrm{SR}=$ Short Rod

TDCB $=$ Tapered Double Cantilever Beam WOF $=$ Work of Fracture

Extensive discussions of measurement methods and their relative merits are given by S. W. Freiman, American Ceramic Society Bulletin, Vol. 67, pp. 392-402 (1988); S. W. Freiman in Fracture Mechanics of Ceramics, Vol. 6, edited by R. C. Bradt et al., Plenum, New York (1983), pp. 27-45; and J. B. Wachtman, Mechanical Properties of Ceramics, John Wiley \& Sons, Inc., New York (1996), chapter six. A summary of the methods noted in the present compilation is given here, along with additional references, as needed.

Many of the test methods involve a notched specimen. To unify the summaries of those methods, let us assume that the specimen, Fig. 3, is a parallellapiped whose pairs of opposite faces may be referred to as (top and bottom), (front and back), and (left side and

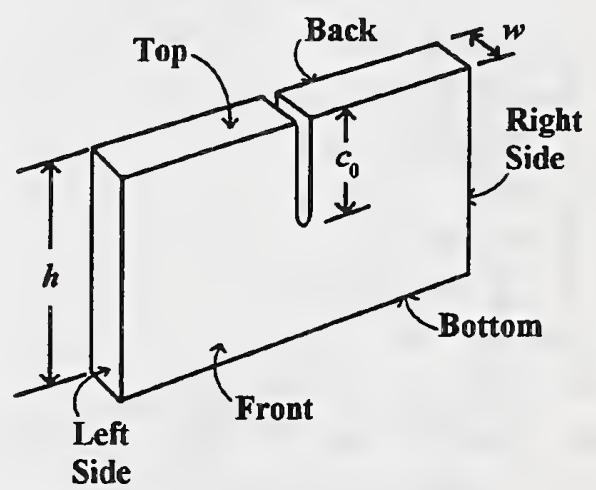

Figure 3: Schematic of a notched specimen 
right side). The notch can then be assumed to be cut into the top surface towards the bottom surface, parallel to the sides, and extending completely across the specimen from the front surface to the back surface. The depth of the cut, $c_{0}$, is less than the distance, $h$, from the top surface to the bottom surface.

\subsection{1 $\mathrm{AMDCB}=$ Applied Moment Double Cantilever Beam}

This method is also known as the constant moment double cantilever beam method. The AMDCB method is similar to the DCB method, but the applied load is replaced by an applied moment, $M$, applied to the top surface. If a load $P$ is applied at a distance $s$ from a fulcrum, then a moment $M=P s$ is generated. A moment, $M$, is applied to each arm of the notched specimen. Then, $K_{\mathrm{Ic}}=$ $M /(I d)^{1 / 2}$, where $I$ is the moment of inertia of a single arm and $d$ is the distance from the front surface to the back surface. The applied stress intensity factor is independent of the initial crack length.

\subsection{2 $\mathrm{CF}=$ Controlled Flaw}

The name "controlled flaw" has been superseded by the name "surface crack in flexure" although the older name still enjoys some usage. The controlled surface flaw and controlled microflaw methods are equivalent also. See SCF for further discussion.

\subsubsection{CNB = Chevron Notch Beam}

The notch is cut in the shape of a chevron, and the specimen is subjected to either threepoint or four-point bending. In three-point bending, the central load point is on the bottom surface under the notch, and the latteral load points are on the top surface; the loads are normal to the top and bottom surfaces. In four-point bending, the central load point on the bottom surface is replaced by a pair of load points, one on each side of the notch.

\subsubsection{DCB = Double Cantilever Beam}

The specimen is notched, and the load is applied normal to the crack plane on each arm of the specimen at the top surface of the specimen.

\subsubsection{DT = Double Torsion}

In the DT method, a notched specimen is loaded at four points. Two of the loads are applied normal to the front surface at the top of the specimen, one load point next to each side of the notch. The other two loads are applied normal to the back surface at the top of the specimen, one load point near to each side of the specimen. Evaluation of $K_{\mathrm{Ic}}$ requires the additional knowledge of the value of Poisson's ratio for the specimen.

\subsection{6 $\mathrm{HI}=$ Hertzian Indentation}

[P. D. Warren, Determining the Fracture Toughness of Brittle Materials by Hertzian Indentation, Journal of the European Ceramic Society, Vol. 15, pp. 201-207 (1995)]

A hard sphere is pressed into the surface of the specimen to generate ring cracks initiating at the edge of the contact zone. Fracture toughness is related to the radius of the ring cracks. Additional knowledge of the elastic modulus and Poisson's ratio is required.

\subsubsection{ICS = Indentation Crack Size} In the ICS method, a diamond indentor is pressed into the surface of the specimen under a known load. The indentation produces radial cracks extending from the 
vertices of the impression, and the resulting crack length can be related to the fracture toughness of the specimen. Additional knowledge of the ratio, $E / H$, of the elastic modulus, $E$, and the hardness, $H$, is required for the evaluation of $K_{\mathrm{Ic}}$.

\subsubsection{IS = Indentation Strength}

The IS method involves two parts. First, an indentation is made in the specimen as in the ICS method. Then, the strength of the indented specimen is measured in four-point bending. The measured strength and the additional knowledge of the ratio, $E / H$, of the elastic modulus, $E$, and the hardness, $H$, are required for the evaluation of $K_{\mathrm{Ic}}$. This method has the advantage that the crack size does not have to be measured.

\subsubsection{NDC $=$ Notched Diametral Compression}

[D. K. Shetty et al., J. Am. Ceram. Soc., Vol. 68, pp. c-325 - c-327 (1985)]

A specimen in the shape of a disk is used in the diametral compression test. The load is applied along a diameter of the disk. The maximum tensile stress develops transverse to the load axis. For the NDC, notches are machined through the thickness of the disk, one notch at each end of the load axis. The diametral configuration has the advantage of simple specimen manufacture. Additionally, values for the elastic modulus and Poisson's ratio are required for the evaluation of $K_{\mathrm{Ic}}$.

\subsubsection{SCF $=$ Surface Crack in Flexure}

[C. A. Tracy and G. D. Quinn, Fracture Toughness by the Surface Crack in Flexure (SCF) Method, Ceramic Engineering and Science Proceedings, Vol. 15, pp. 837-845 (1994)]

In the SCF method, an indentation is made in the surface of the specimen, as in the IS method, to create an initial crack (called a precrack). The surface is then lapped to remove the residual surface impression leaving only the precrack in the surface. The strength of the specimen is then determined in four-point bending. The size of the precrack is determined by post-test fractography. $K_{\mathrm{Ic}}$ is evaluated from the measured strength, the size of the precrack, and a geometric shape factor.

\subsubsection{SENB = Single-Edge Notched-Beam} A straight notch is cut by means of a saw blade or a wire, and the specimen is subjected to either three-point or four-point bending. In three-point bending, the central load point is on the bottom surface under the notch, and the latteral load points are on the top surface; the loads are normal to the top and bottom surfaces. In four-point bending, the central load point on the bottom surface is replaced by a pair of load points, one on each side of the notch. SENB results may overestimate $K_{\mathrm{Ic}}$ and may have lower reproducibility than other methods because the notch tip is not sharp.

\subsubsection{SEPB $=$ Single-Edge Precracked Beam}

SEPB is a variation on the SENB test. Prior to testing, the specimen is stressed so that the notch is extended in the form of a sharp crack. Additional post-test fractography is required to assess the initial crack size.

\subsubsection{SR = Short Rod}

[L. M. Barker, Engineering Fracture Mechanics, Vol. 9, pp. 361-369 (1977)] The SR method is a variation on the DCB method. The SR specimen is a rod with a diameter that is typically two-thirds the size of the length. A V-shaped notch is cut into 
one end of the rod. A pretest load creates an initial crack in the specimen in the region of the vertex of the V-shaped notch. The precrack is stable if the crack length is less than a critical length, $a_{\mathrm{c}}$. A distinctive feature of this test is that $a_{\mathrm{c}}$ is essentially independent of the material being tested if the size of the plastic zone in the crack region is small compared to the dimensions of the specimen. Once $a_{\mathrm{c}}$ is determined for a given configuration, the fracture toughness is determined by the peak load at fracture.

\subsubsection{TDCB = Tapered Double Cantilever Beam \\ [C. St. John, The Brittle-to-Ductile} Transition in Pre-Cleaved Silicon Single Crystals, Philosophical Magazine, Vol. 32, pp. 1193-1212 (1975).]

The TDCB method is similar to the DCB method except that the sides of the specimen are tapered such that the distance between the sides at the top surface is less than the distance at the bottom surface. The tapered configuration produces a higher stress intensity for lower applied stress than in the DCB configuration; the result is a reduction of plasticity in regions away from the crack tip.

\subsubsection{WOF $=$ Work of Fracture}

[H. G. Tattersall and G. Tappin, Journal of Materials Science, Vol. 1, pp. 296-301 (1966).]

The WOF method is similar to the CNB method except that the load $v$ s. displacement curve is measured and integrated to determine the work done in fracture. However, since this procedure determines the total work of fracture, there is an open question of how this value is related to $\gamma$. WOF values are included in this compilation for completeness.

\subsection{Property Symbols}

The following symbols are used in the comments portion of the data tables.

$K_{I c}=$ Fracture toughness

$E=$ Elastic modulus (Young's modulus)

$H=$ Hardness (Vickers, unless noted)

$v=$ Poisson's ratio

$T c=$ Superconducting critical temperature

\subsection{Units}

SI units, unit symbols, and unit prefixes are used exclusively. For a comprehensive discussion of the use of SI units, see "Guide for the Use of the International System of Units (SI)," by B. N. Taylor, NIST Special Publication 811.

\subsection{Uncertainties}

No attempt has been made to assign uncertainties to the individual values obtained from the literature. A survey of data in the NIST Structural Ceramics Database [Standard Reference Data Program, NIST, Gaithersburg, Maryland 20899] indicates that relative combined standard uncertainties in the range of $5 \%$ to $15 \%$ are not unusual for fracture toughness measurements. Exceptional cases having reported uncertainties as low as $1 \%$ or as high as $30 \%$ can be found, but such cases are very unusual. For a comprehensive discussion on estimates of uncertainty, see "Guidelines for Evaluating and Expressing the Uncertainty of NIST Measurement Results," by B. N. Taylor and C. E. Kuyatt, NIST Technical Note 1297. 


\section{Index of Materials}

Material Designation

ADP [ammonium dihydrogen phosphate] AlN.

$\mathrm{Al}_{2} \mathrm{O}_{3}$

generic

beta.

sapphire

AD-85

AD-90

AD-94

$\mathrm{AD}-96$

$\mathrm{AD}-995$

AD-999

AlSiMag 614

GMB-352

GMB-395

Lucalox

Lucalox-HS.

McDonel 598

Monofrax A

Monofrax M

Vistal.

XA16.

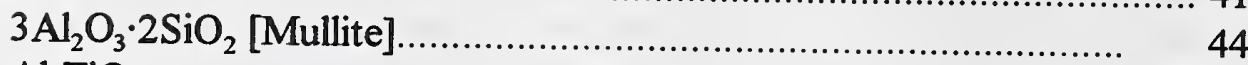

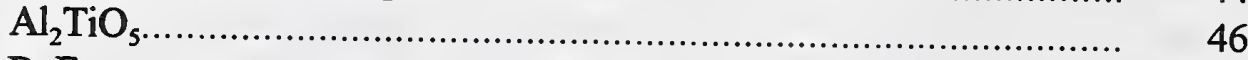

$\mathrm{BaF}_{2}$

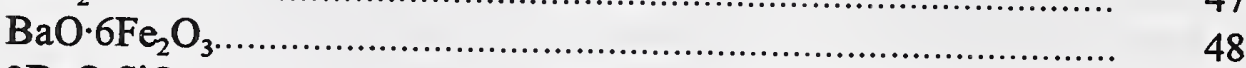

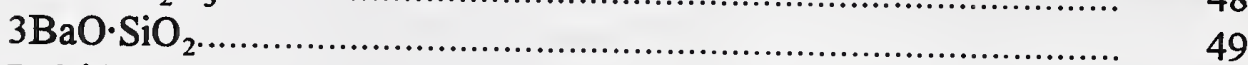

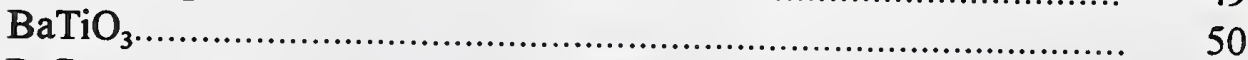

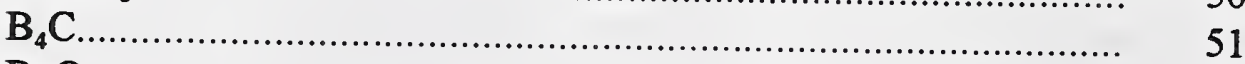

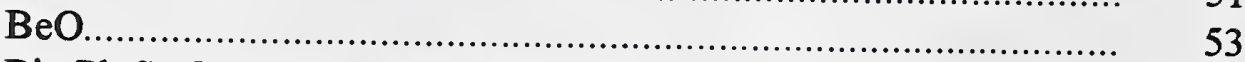

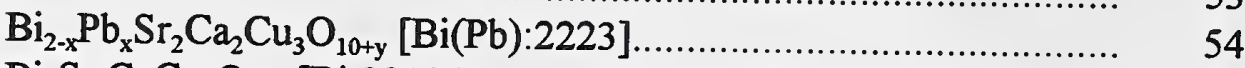

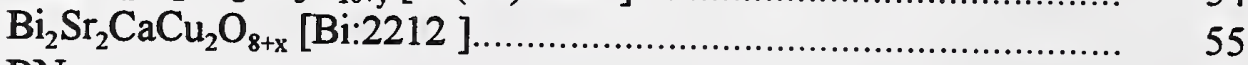

$\mathrm{BN}$

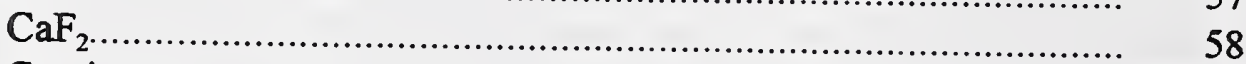

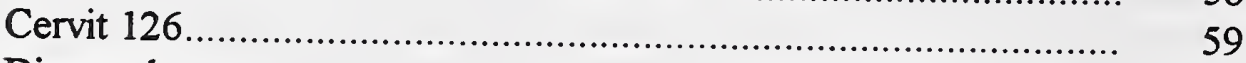

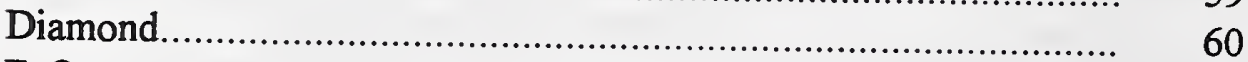

$\mathrm{FeO}$.

$\mathrm{Fe}_{2} \mathrm{O}_{3}$

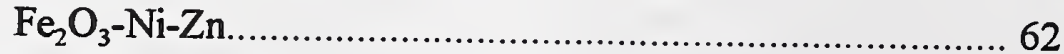

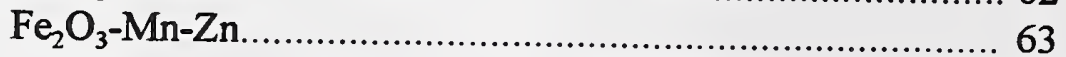

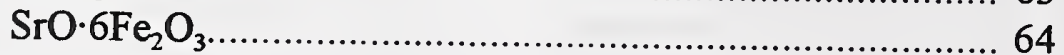




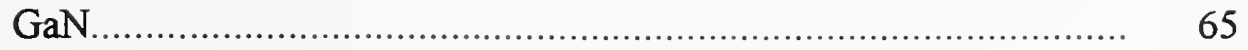

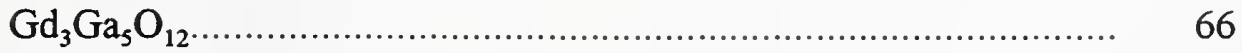

Graphite

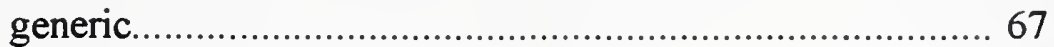

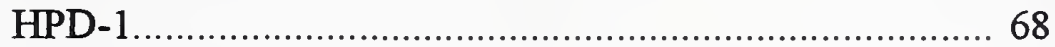

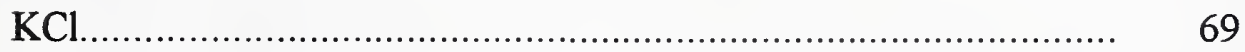

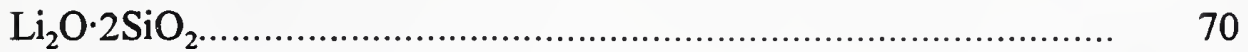

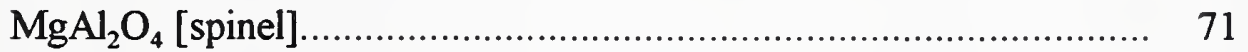

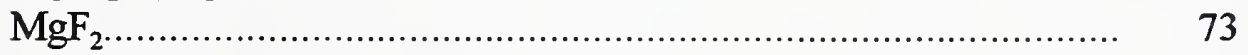

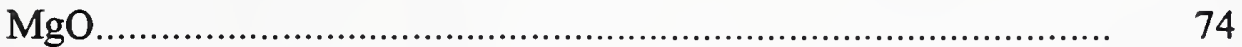

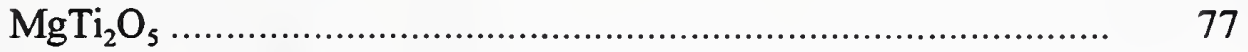

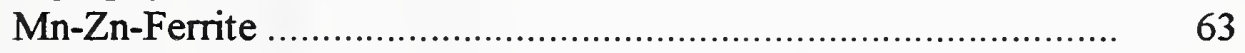

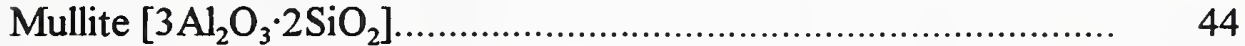

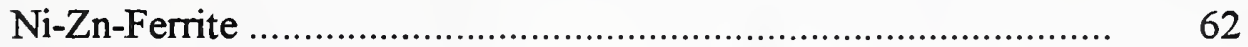

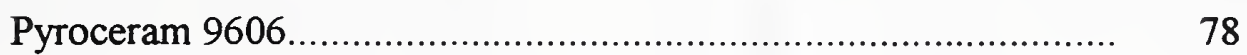

PZT [lead zirconate titanate] ................................................ 80

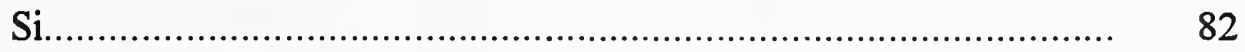

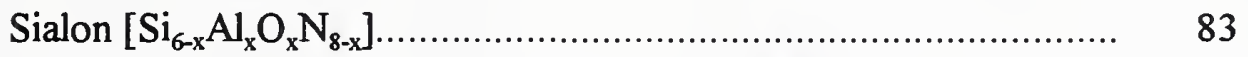

$\mathrm{SiC}$

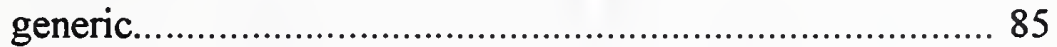

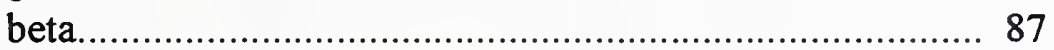

CVD

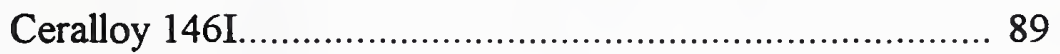

Hexoloy SA........................................................ 90

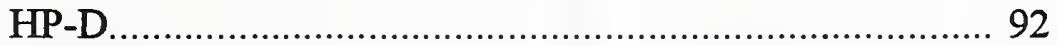

KT

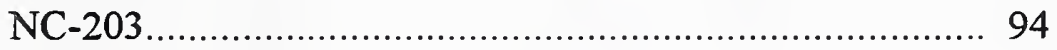

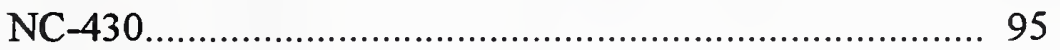

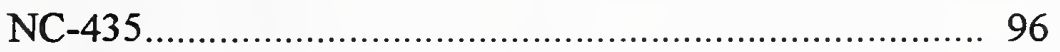

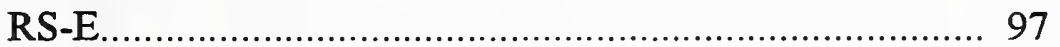

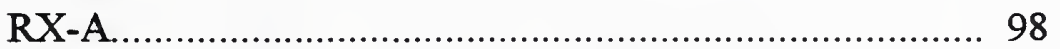

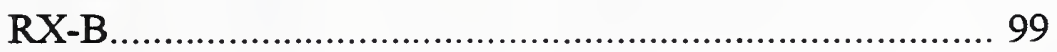

RX-C

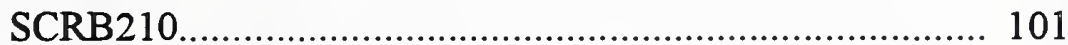

$\mathrm{Si}_{3} \mathrm{~N}_{4}$

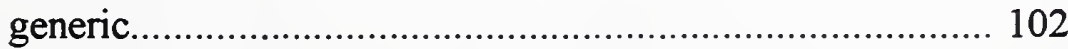

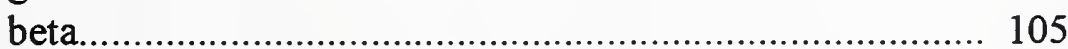

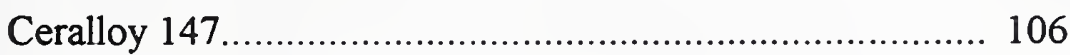

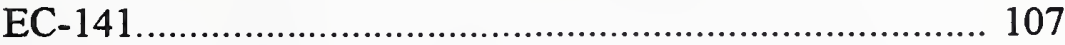

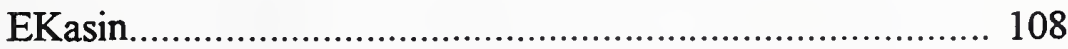

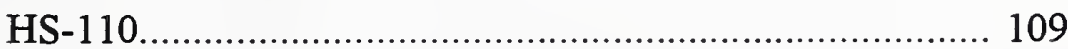

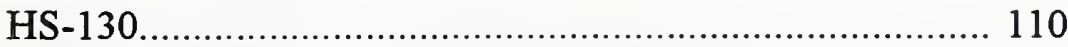

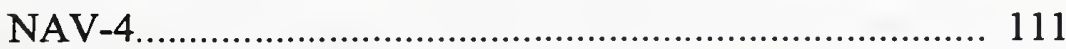




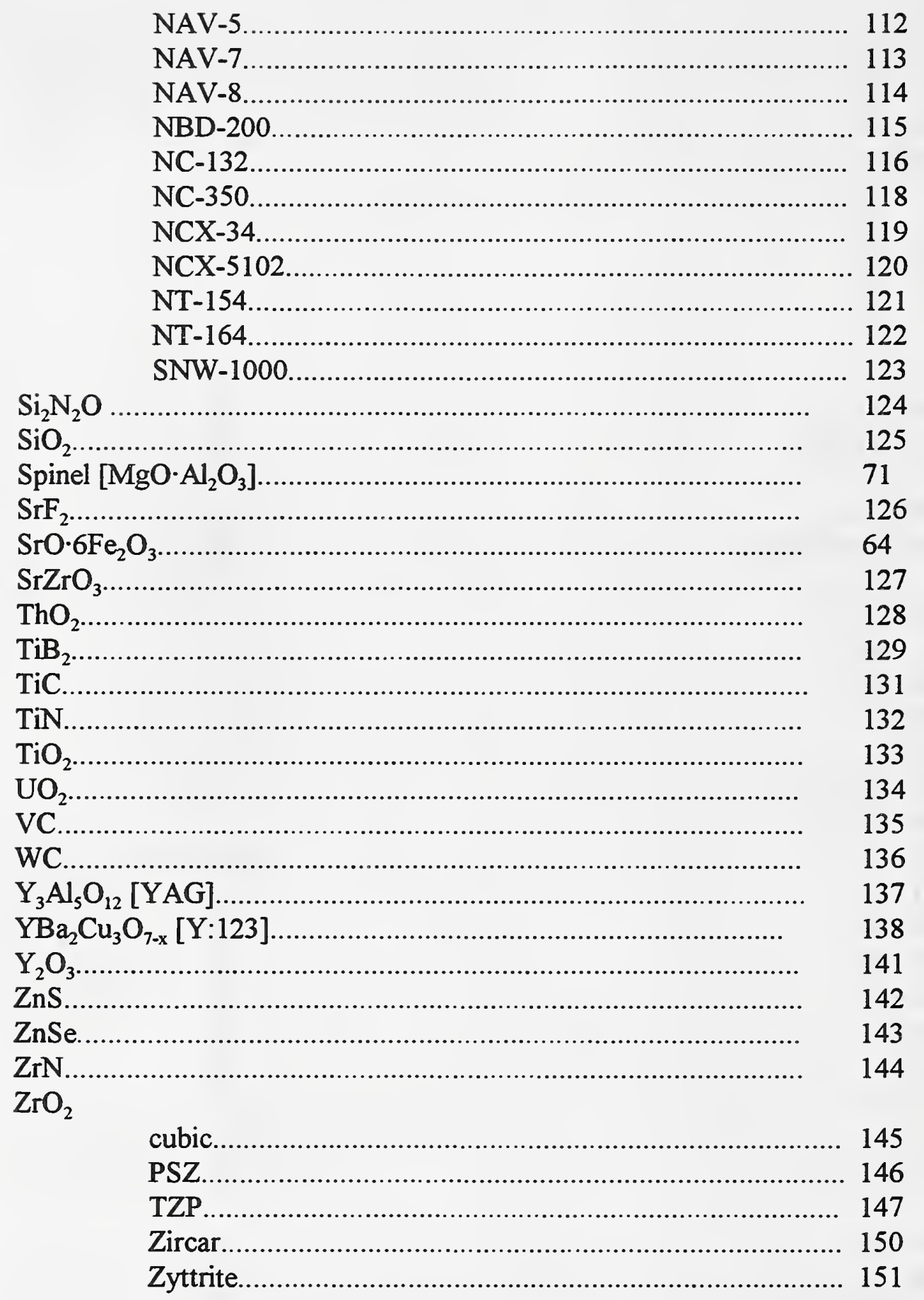

\section{Tables of Data}

Data for all of the materials listed in the preceding section are given on the following pages. 


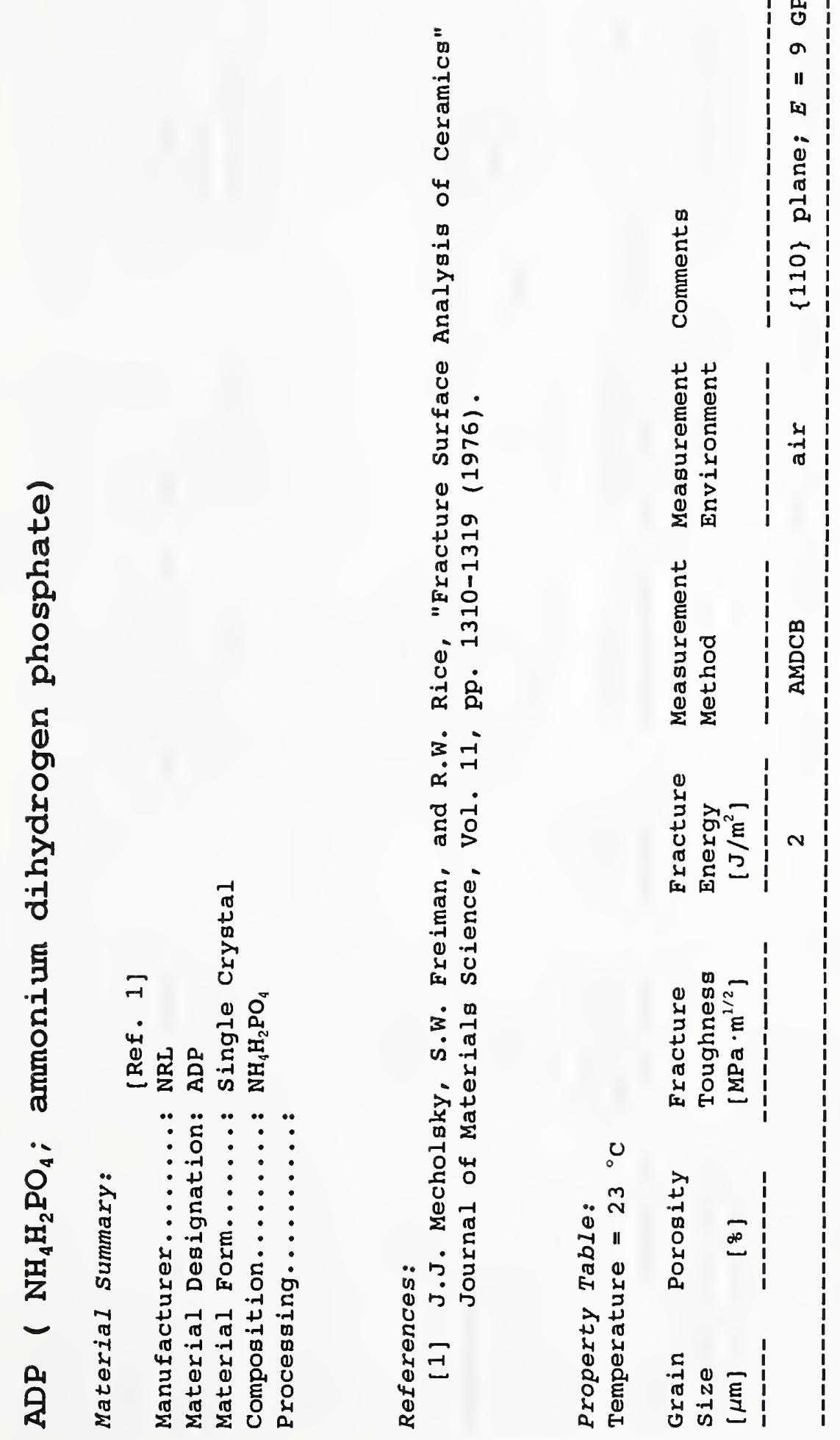




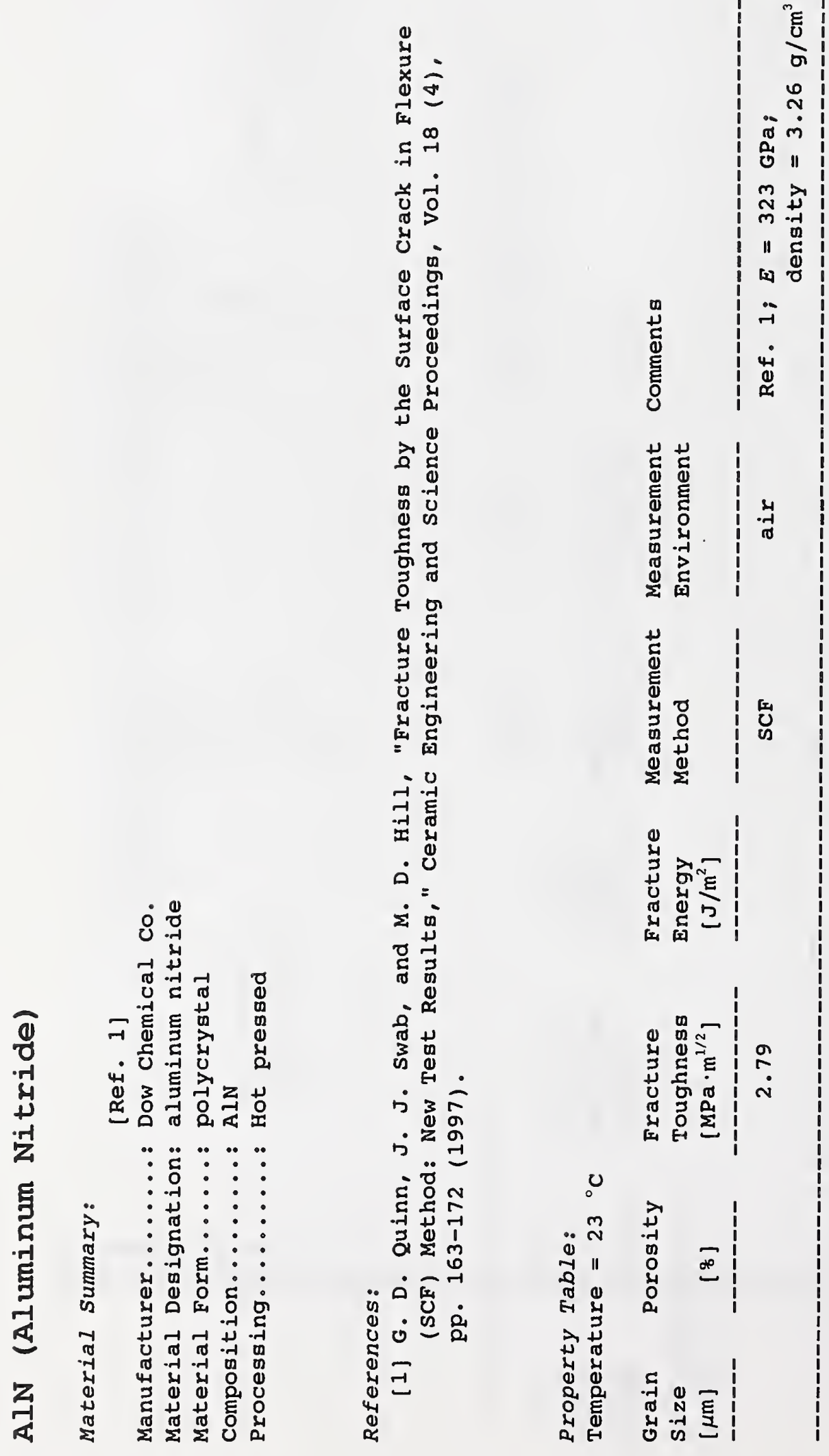




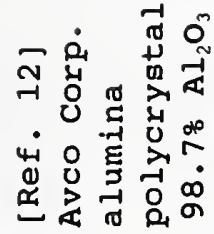

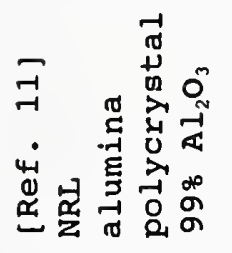

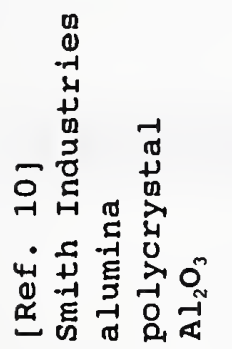

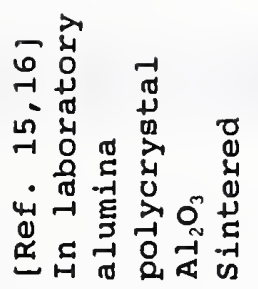

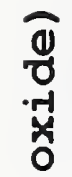

ต

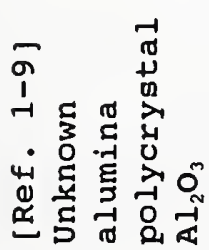$$
\text { : }
$$

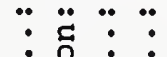

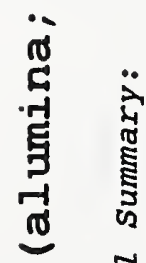

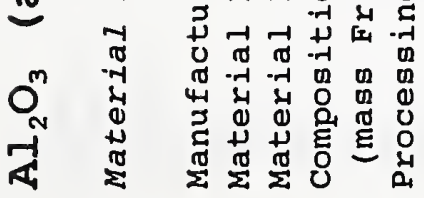
告

$\because . \bullet$

$\ddot{0} \ddot{0}: \ddot{:}:$

$:-11::$

$\vdots$ 苛 $\vdots \vdots$

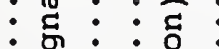

-न ह :

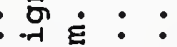

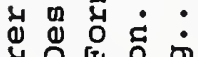

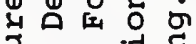

गै-

0 西

世

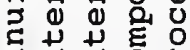

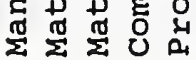

क $\dot{0}=\stackrel{-10}{0}$

مै = हो

() है

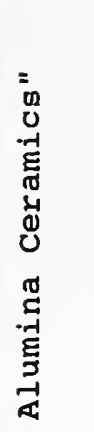

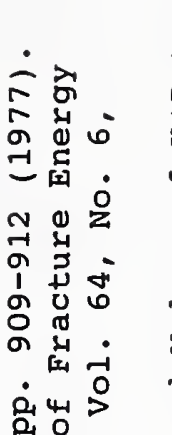

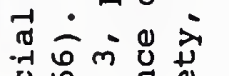

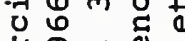

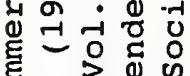

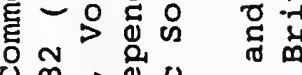

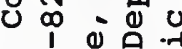

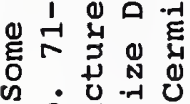

罂

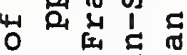

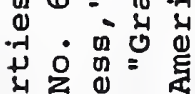

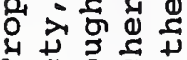

क.

$\Rightarrow$ - $\begin{gathered}-1 \\ 0\end{gathered}$

ठำ

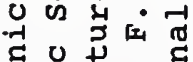

龸

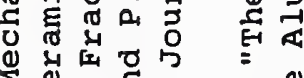

on

点出

嵌

禹

4

वृ क्ष

造

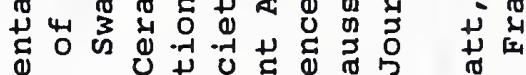

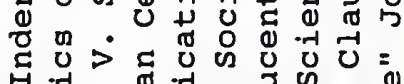

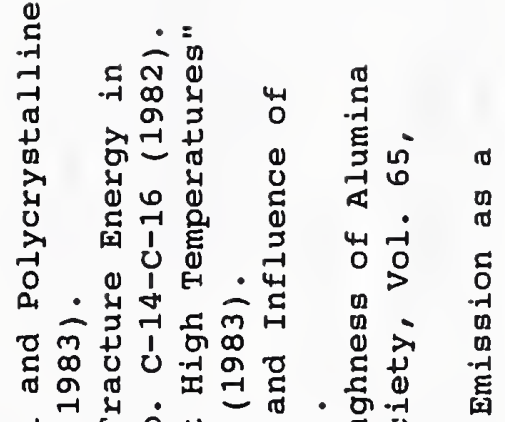

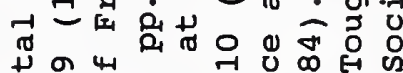

只只

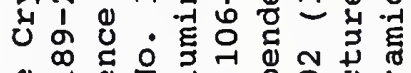

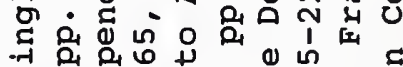

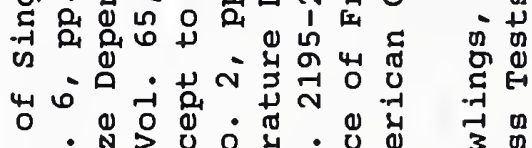

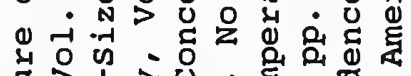

उै।

का का

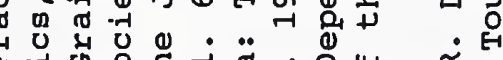

E气 O

$\dot{4}$

至

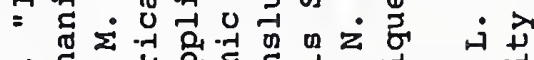

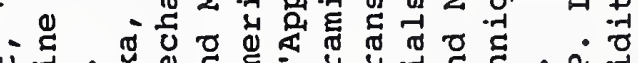

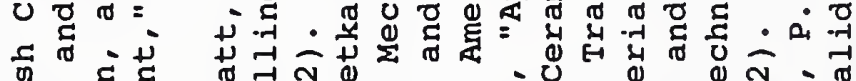

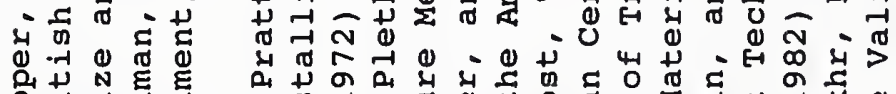

Q

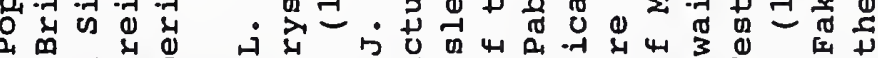

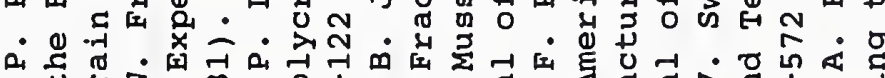

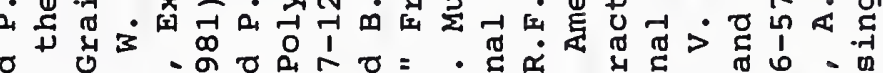

范出

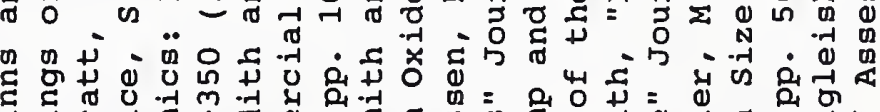

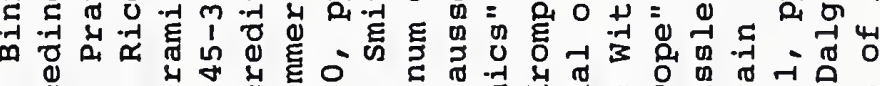

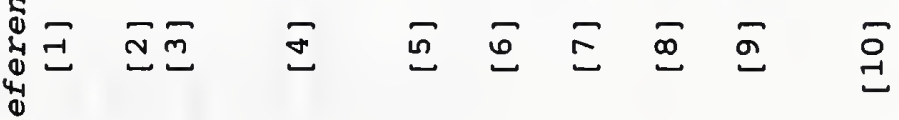

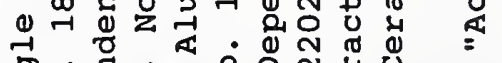

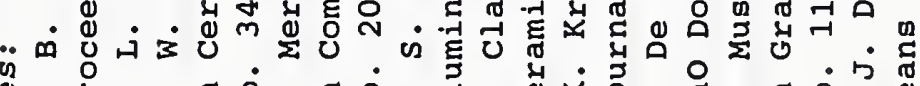

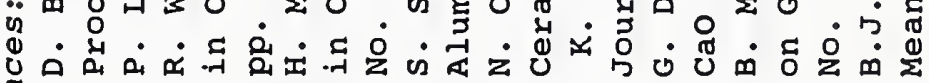



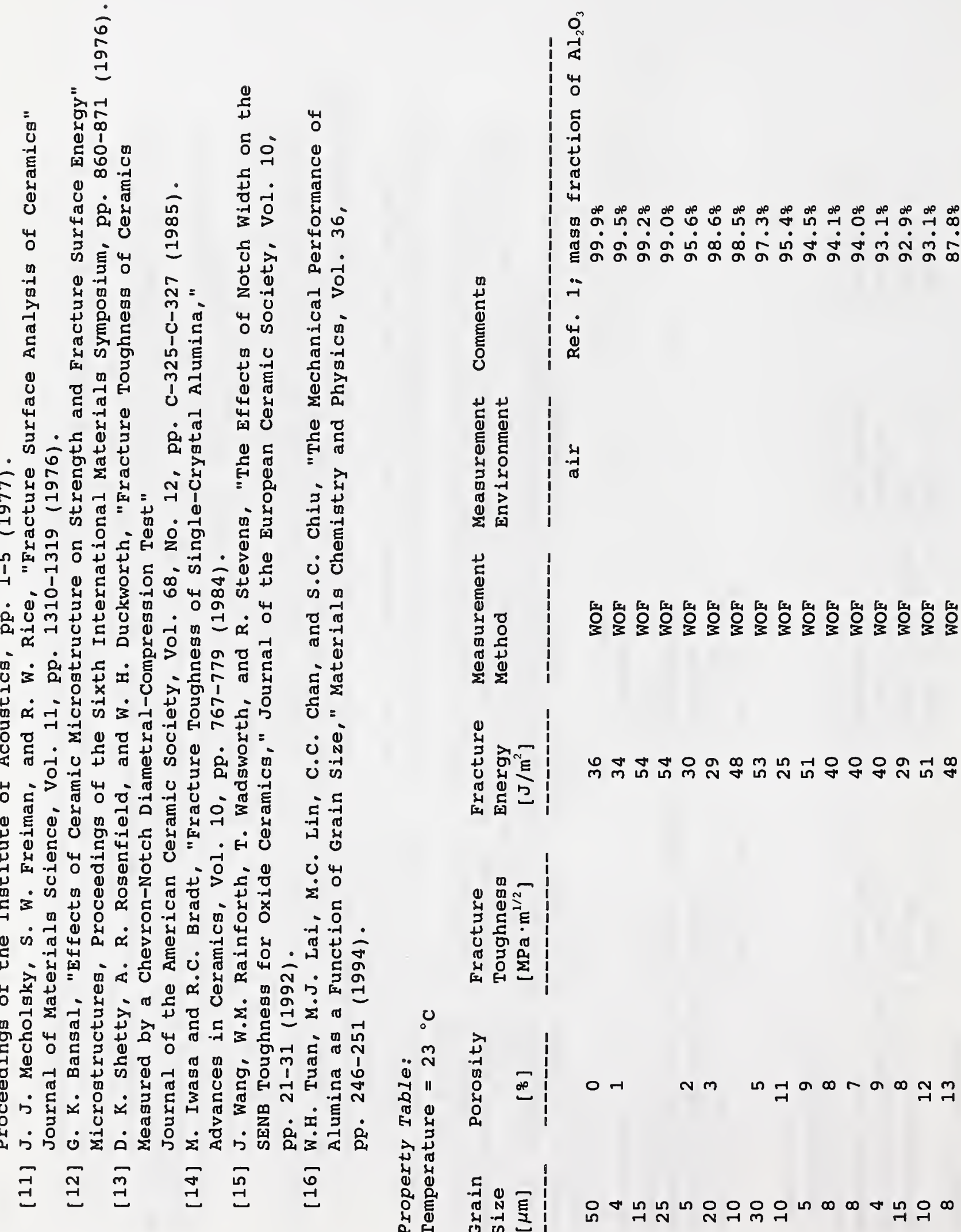

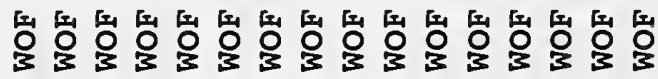

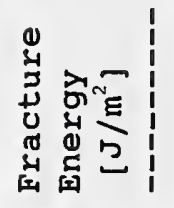

m
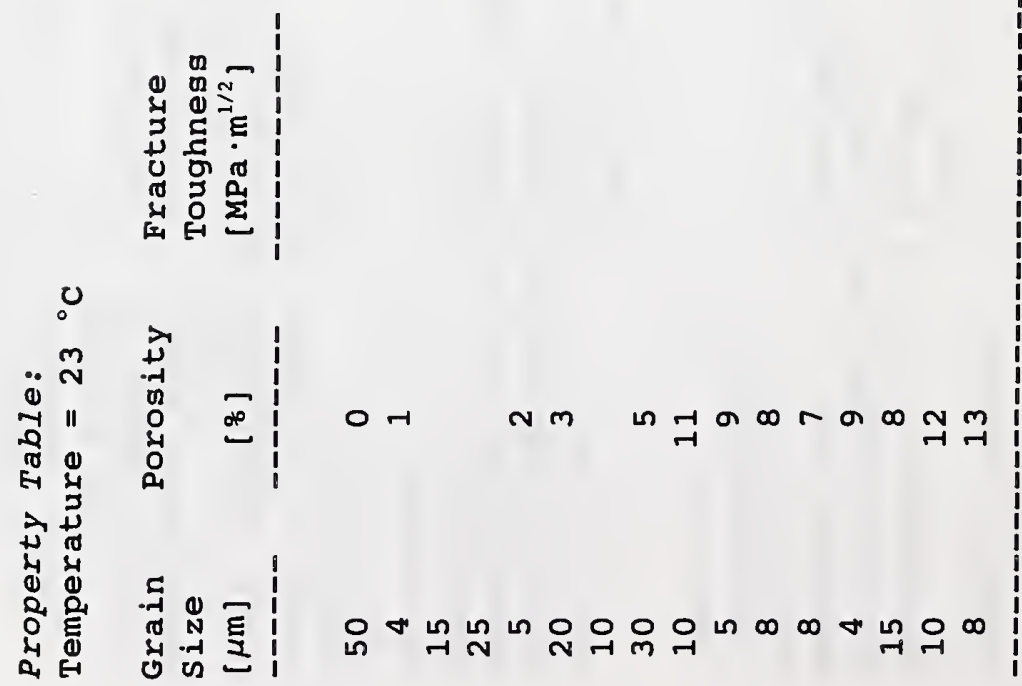

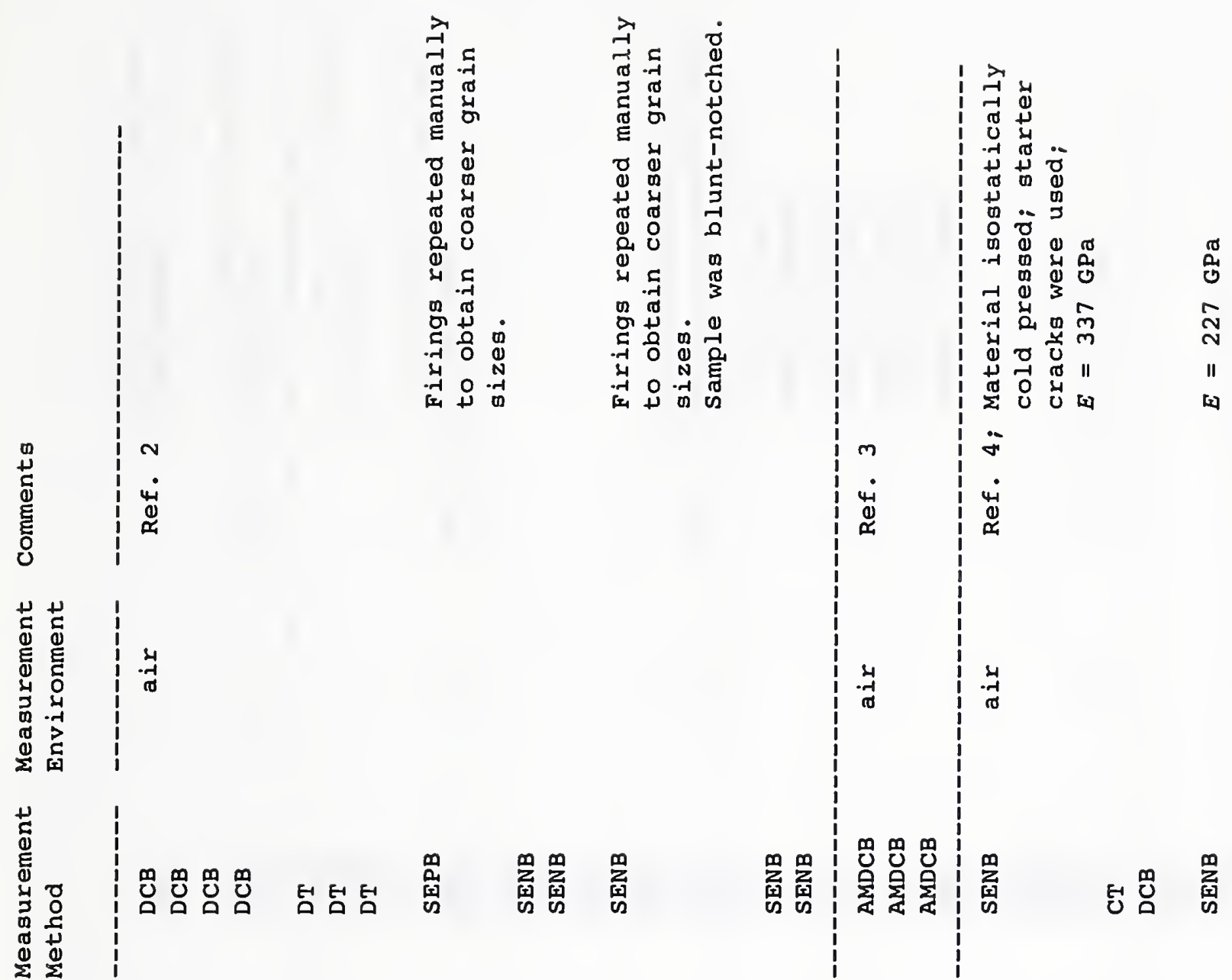

岁

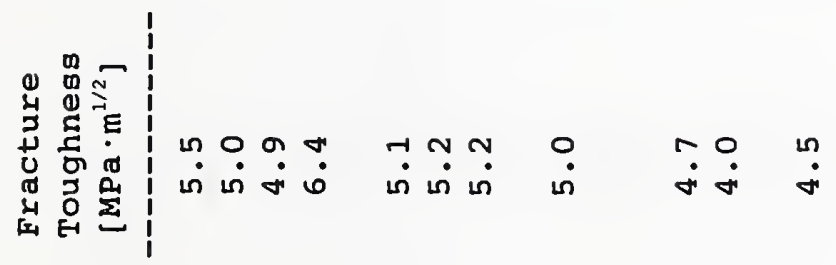

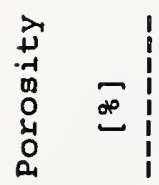

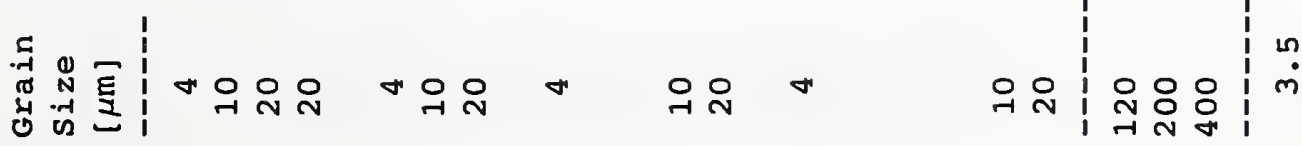

둥우 $\stackrel{m}{\sim} \underset{m}{\sim}$

$\dot{1} \dot{\theta} \dot{m} \dot{m}$

a

$\stackrel{0}{0}$ 


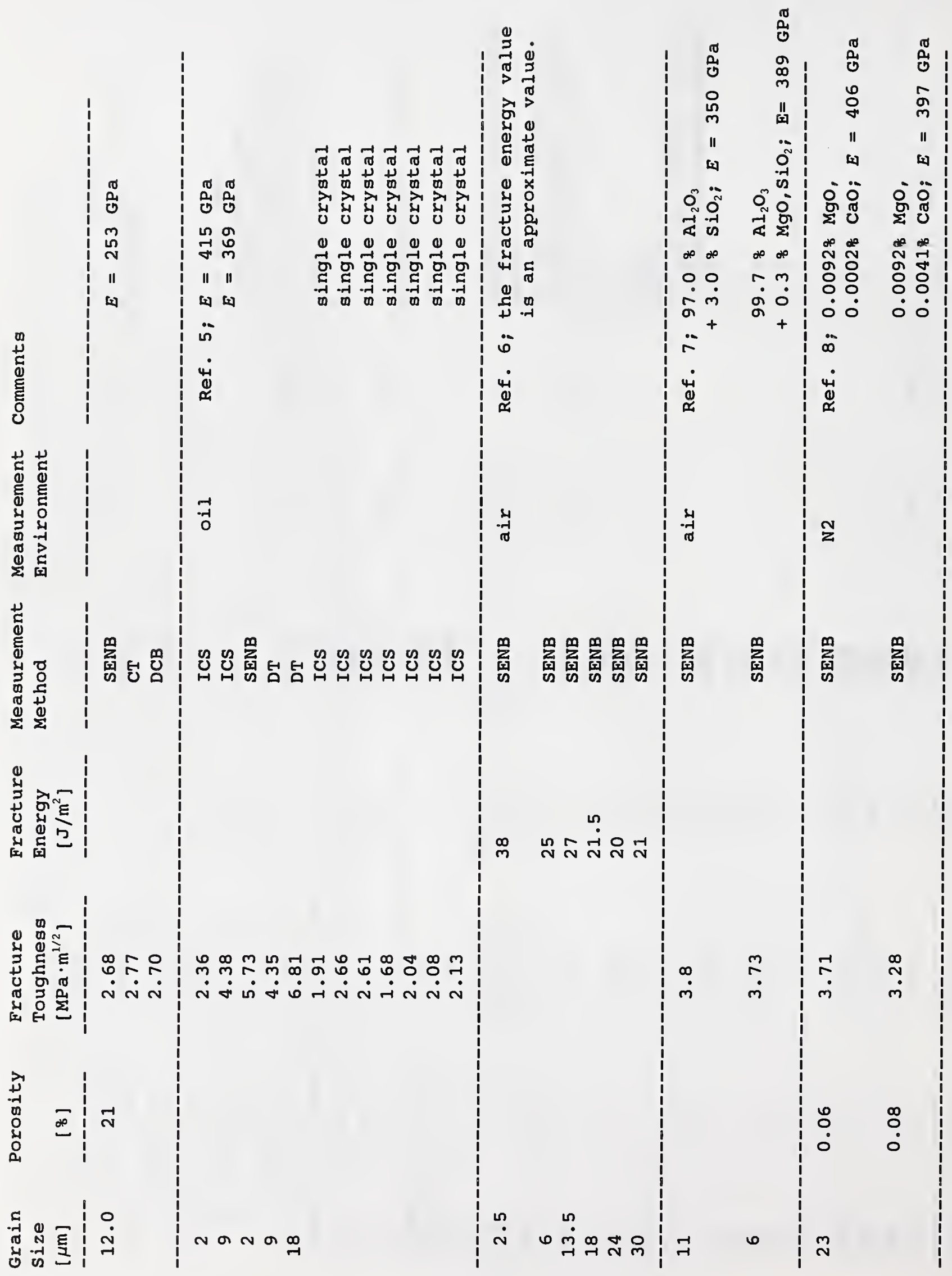



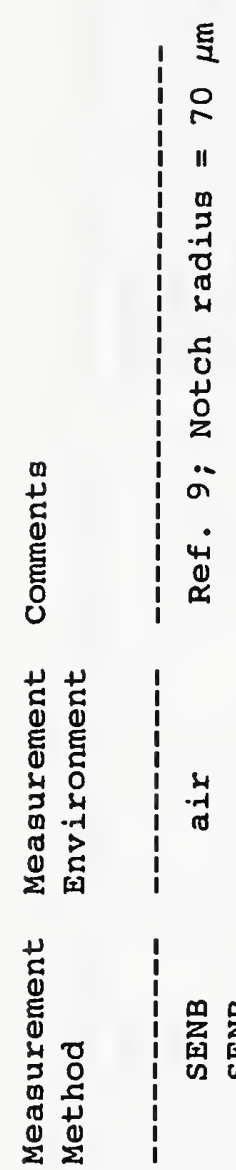

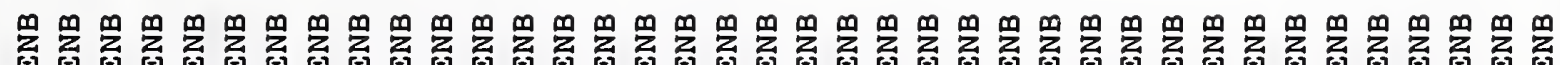

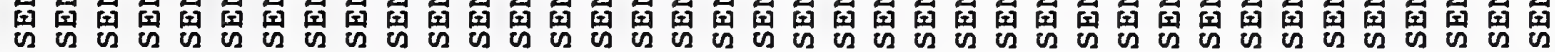
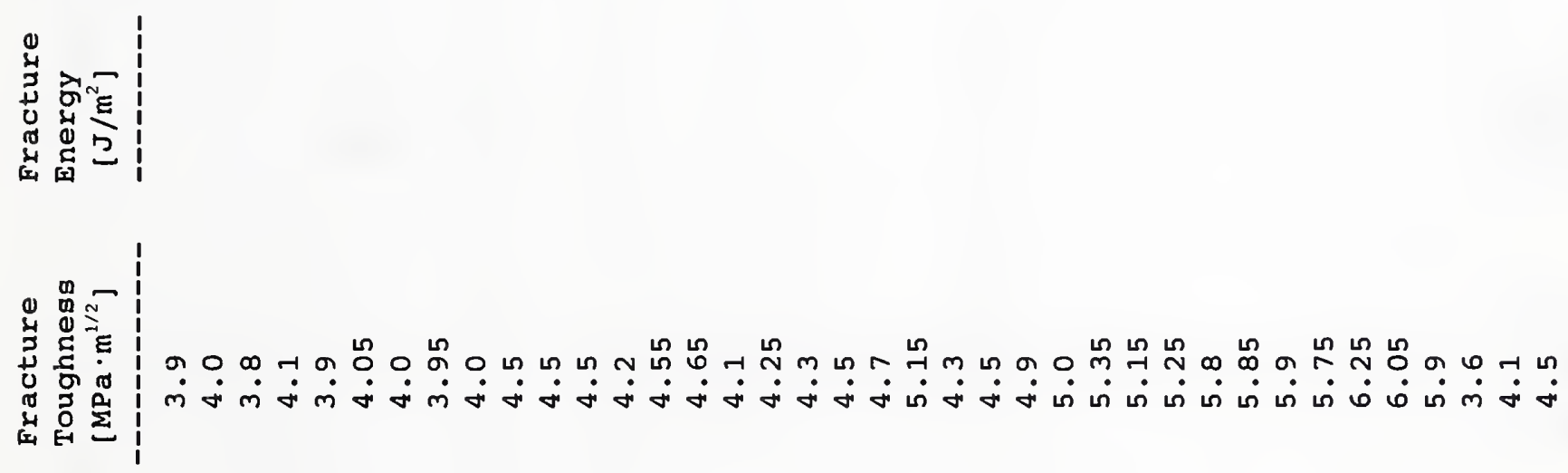

+1
+1
0
0
0
0
0

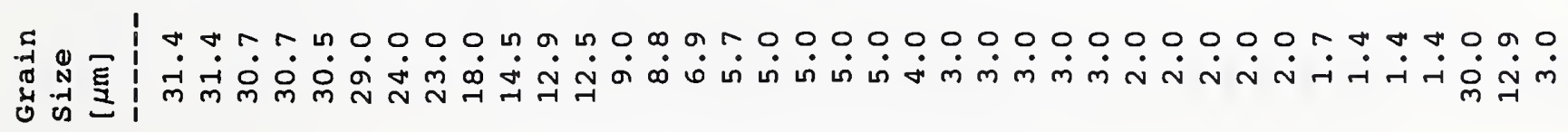




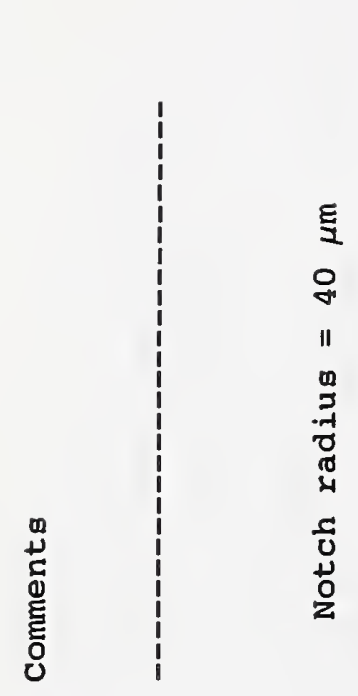

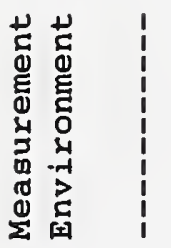

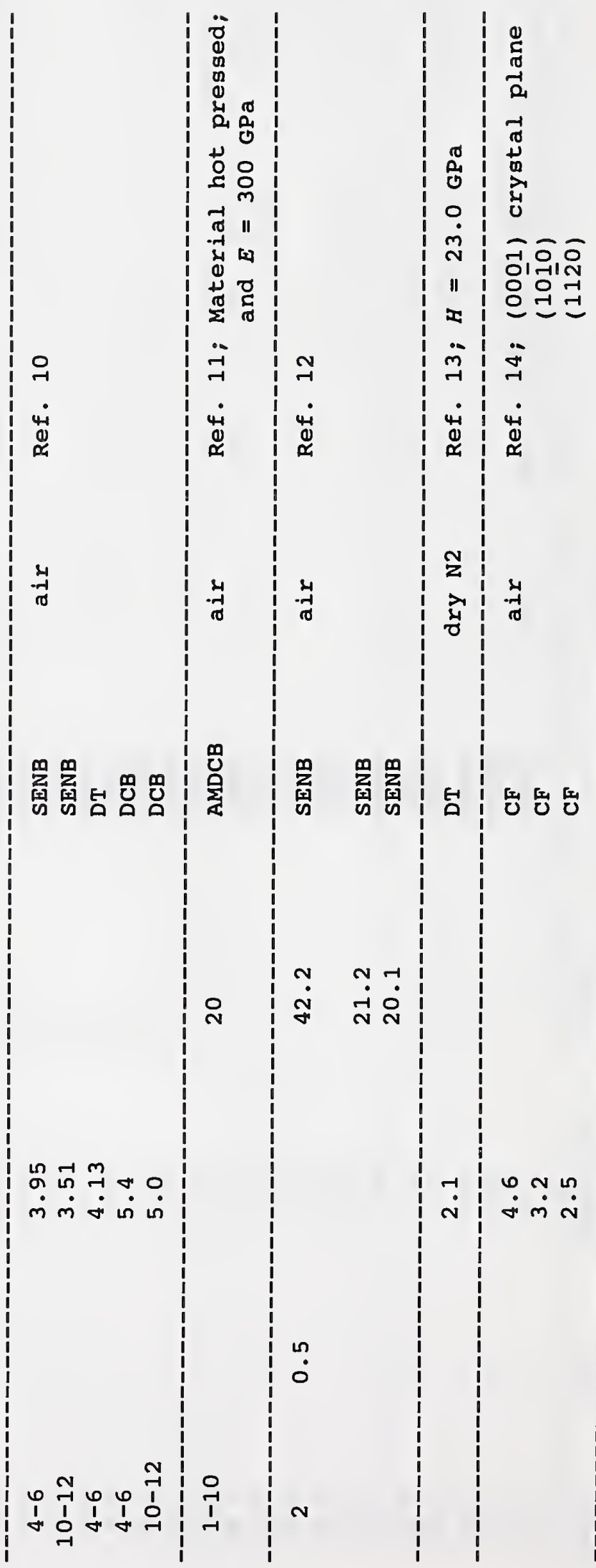

$\stackrel{\infty}{1}$
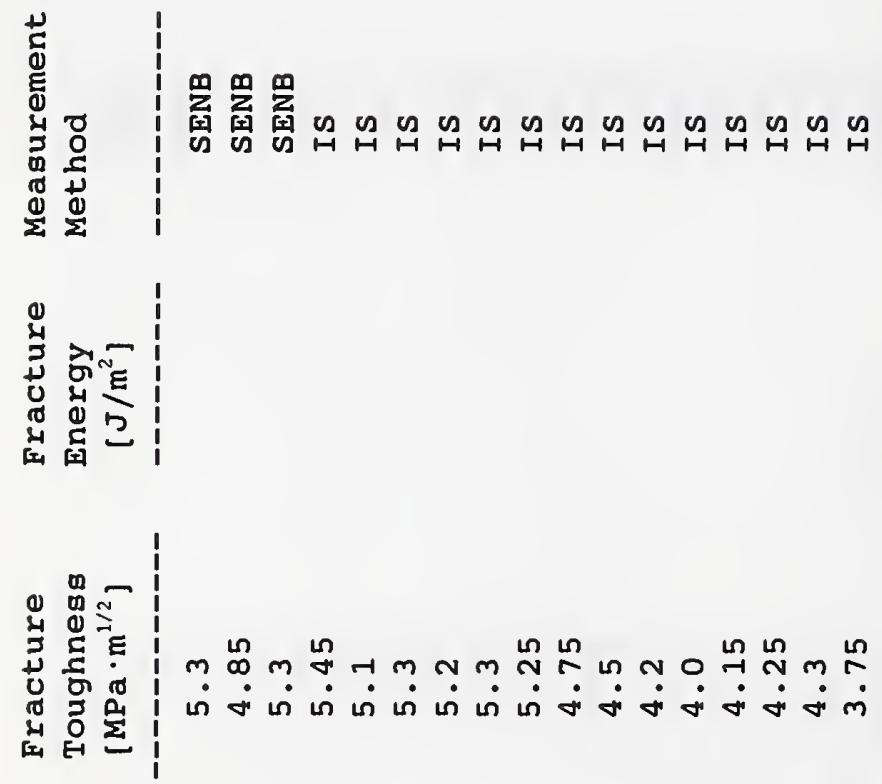

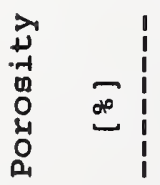

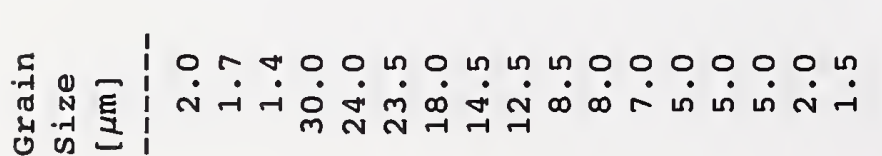




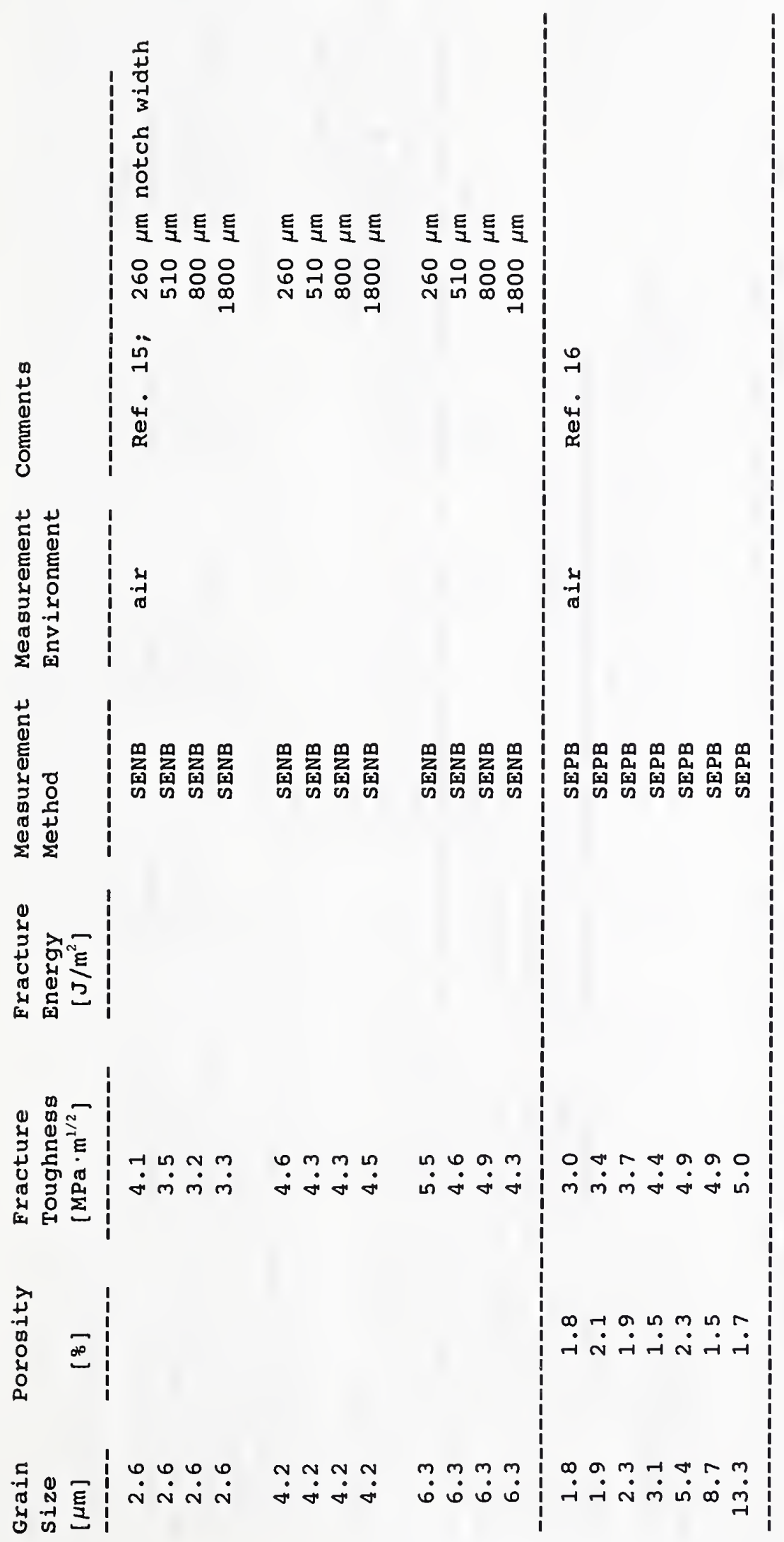




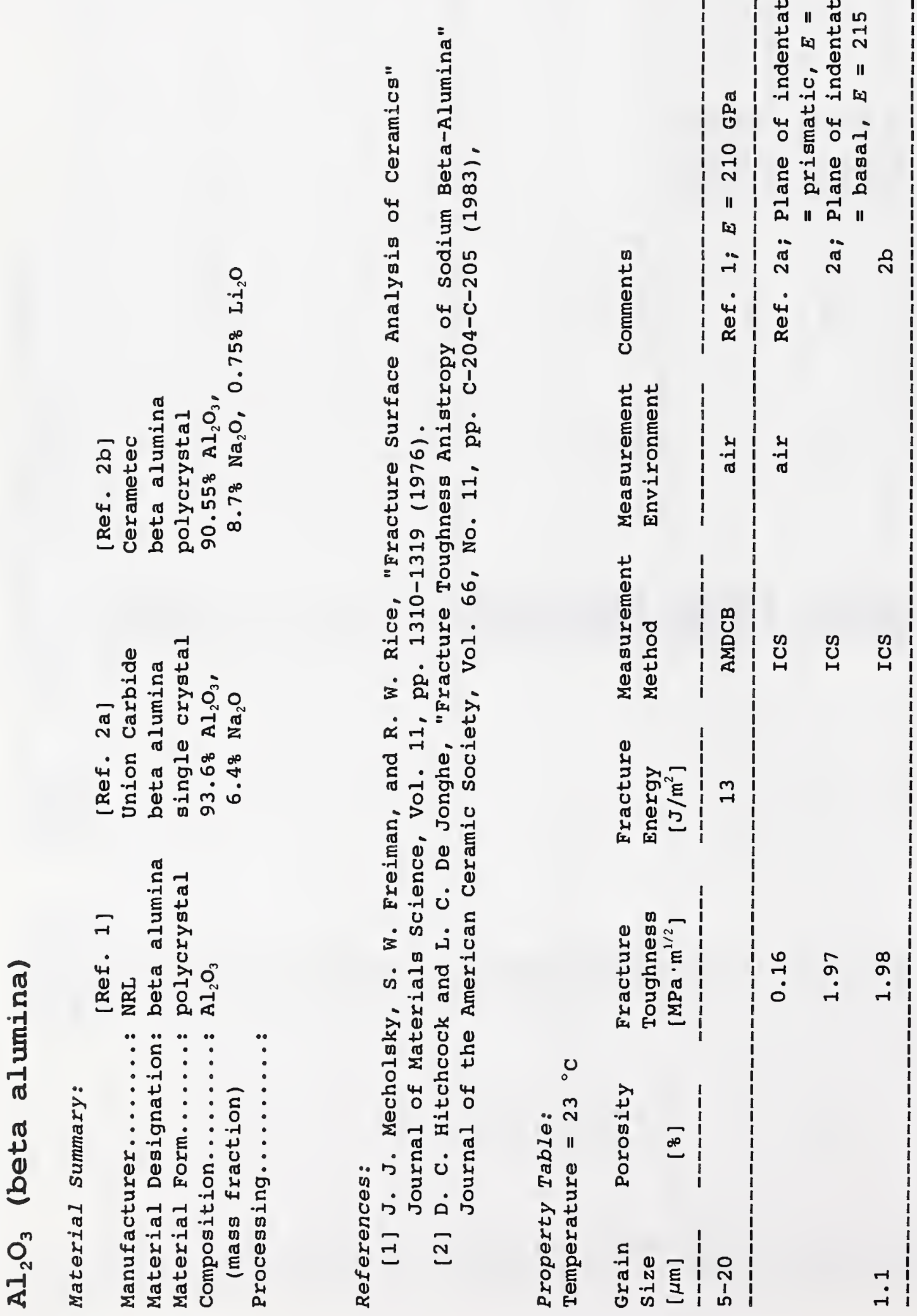



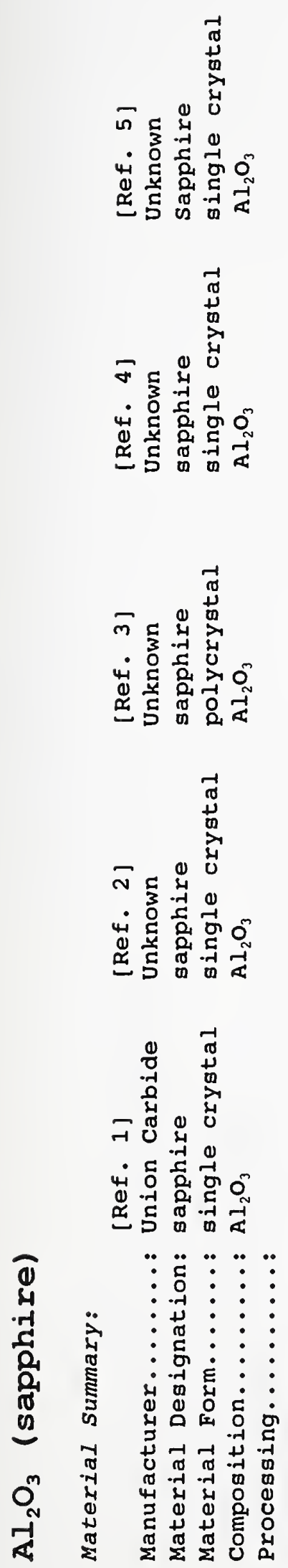
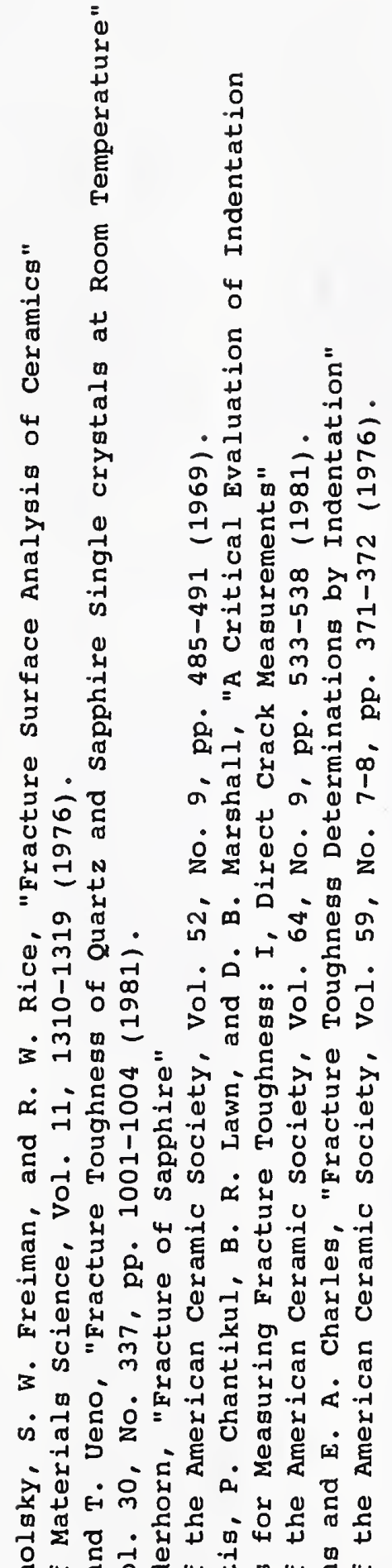

过

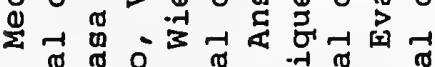

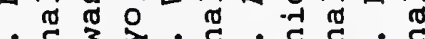
i) 出 U

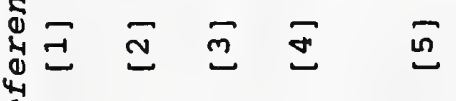




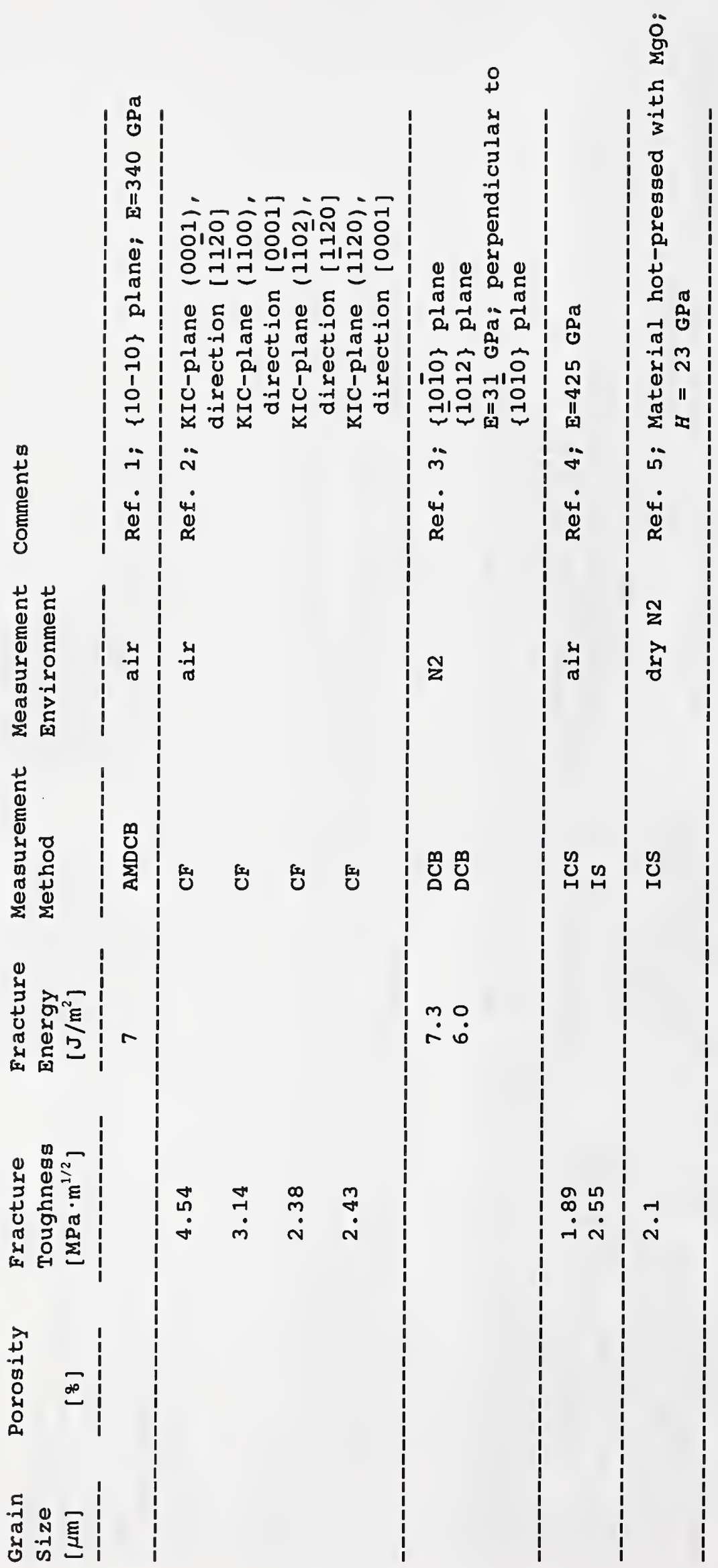




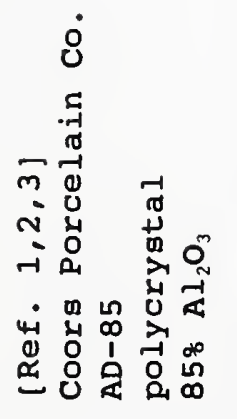

品
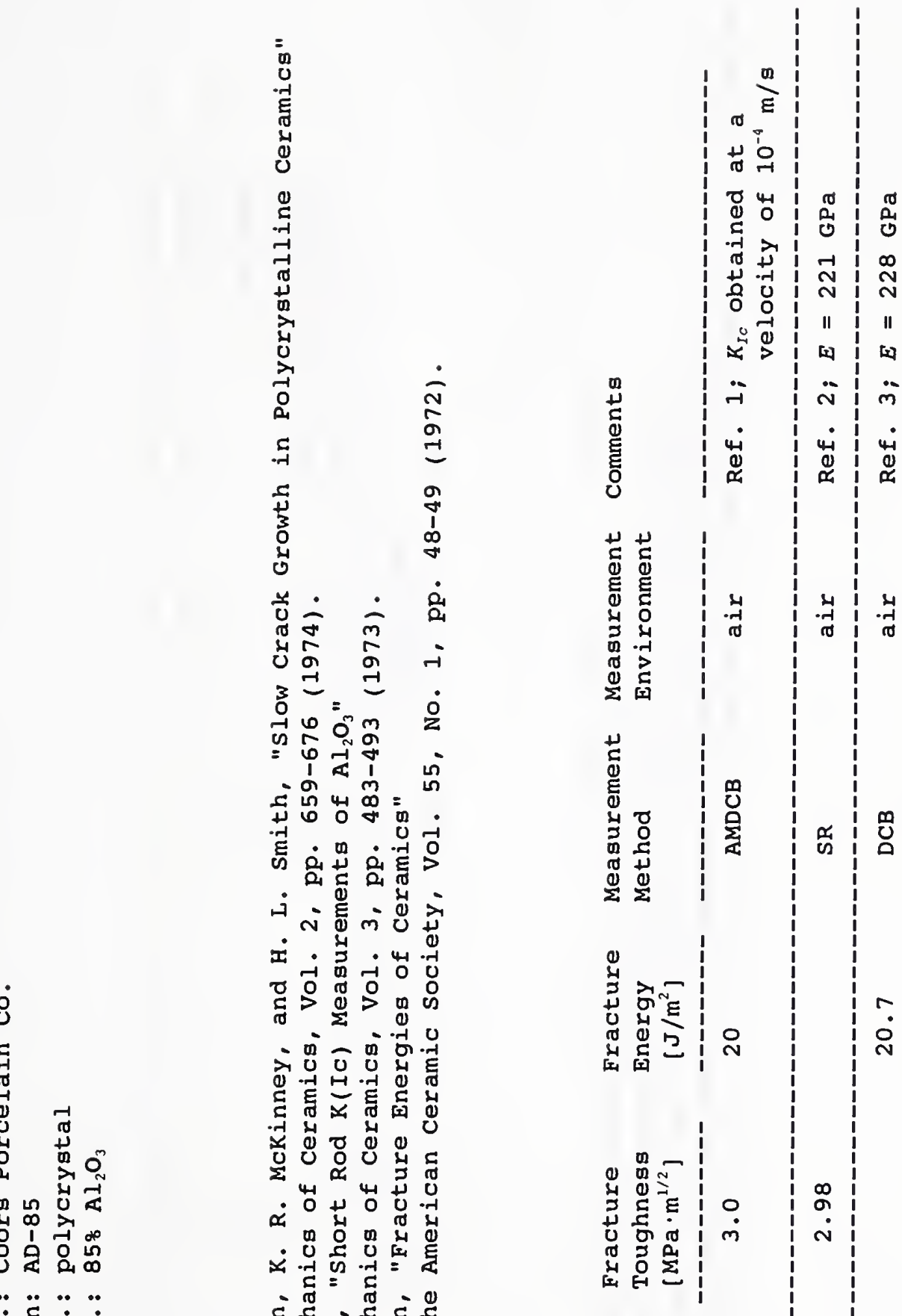

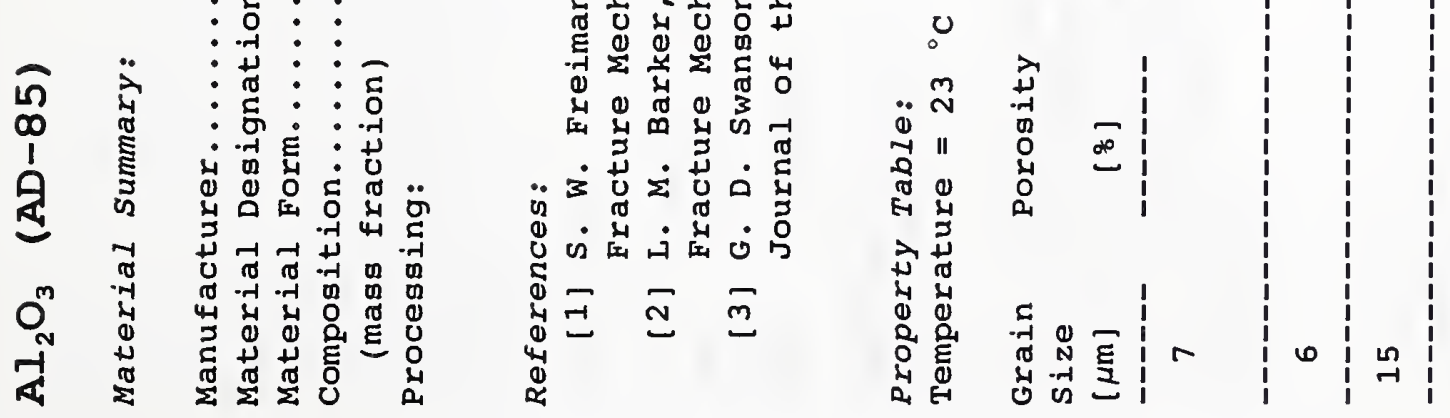

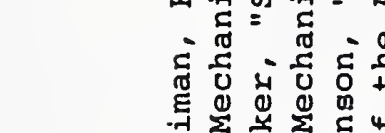




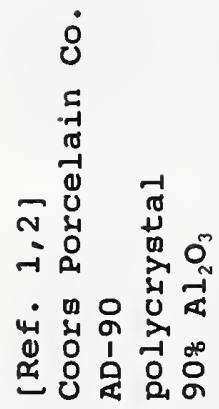

.

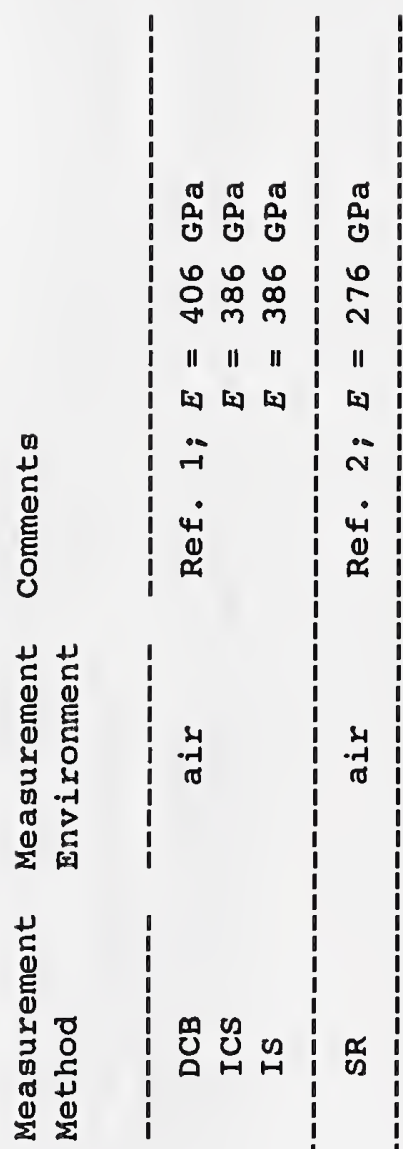

- 空

ई

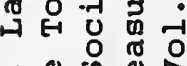

- o क क

4 मू

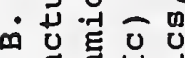

-

न1

至

范

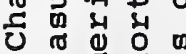

- 运芯的

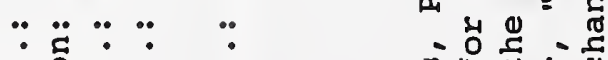

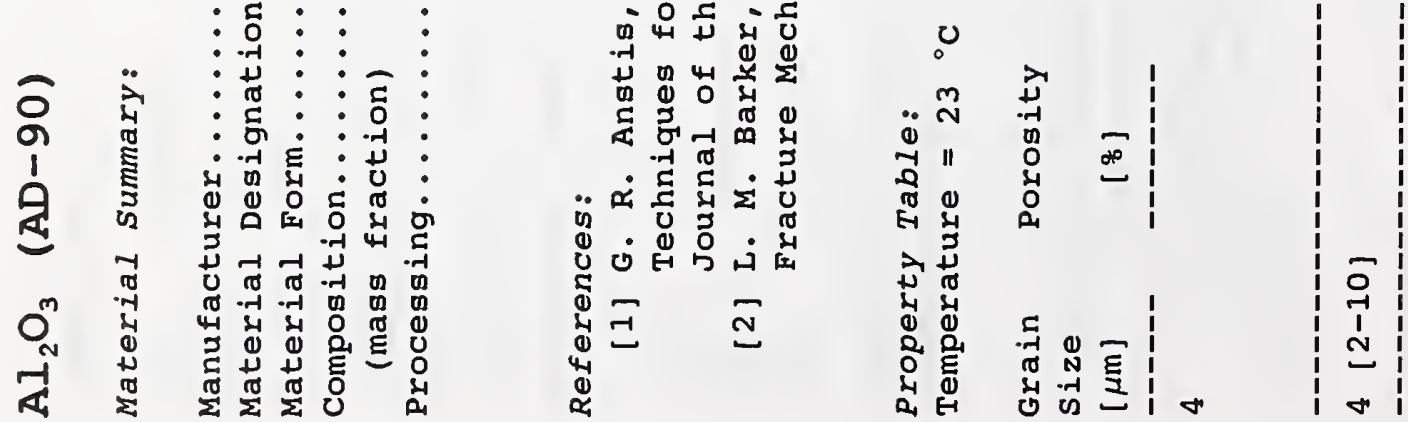
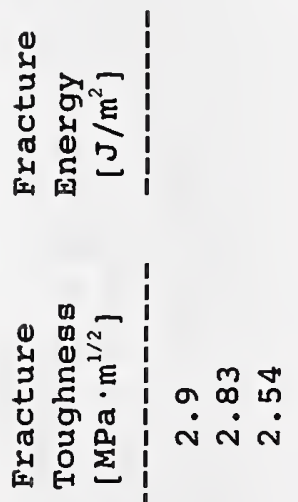

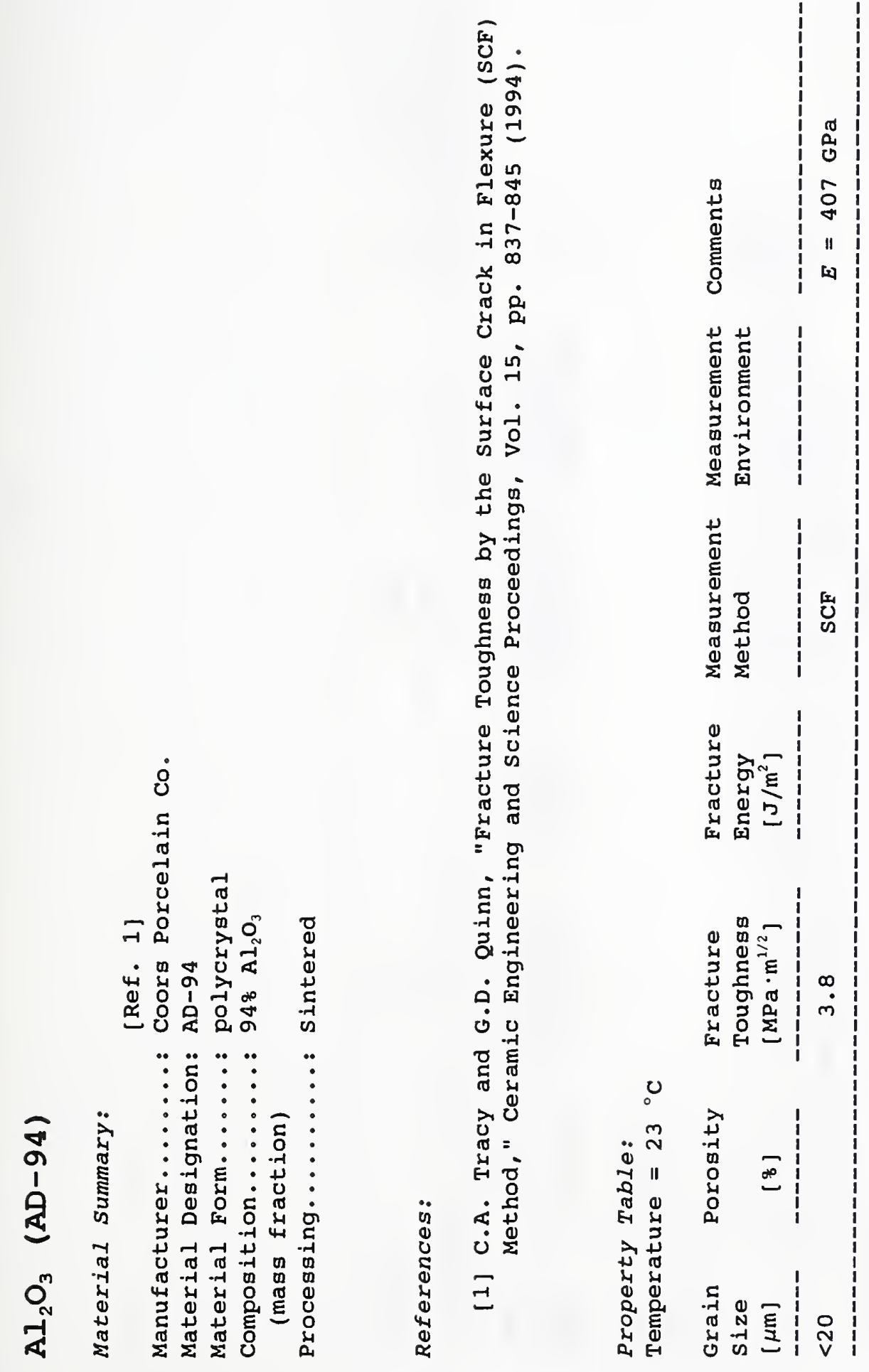

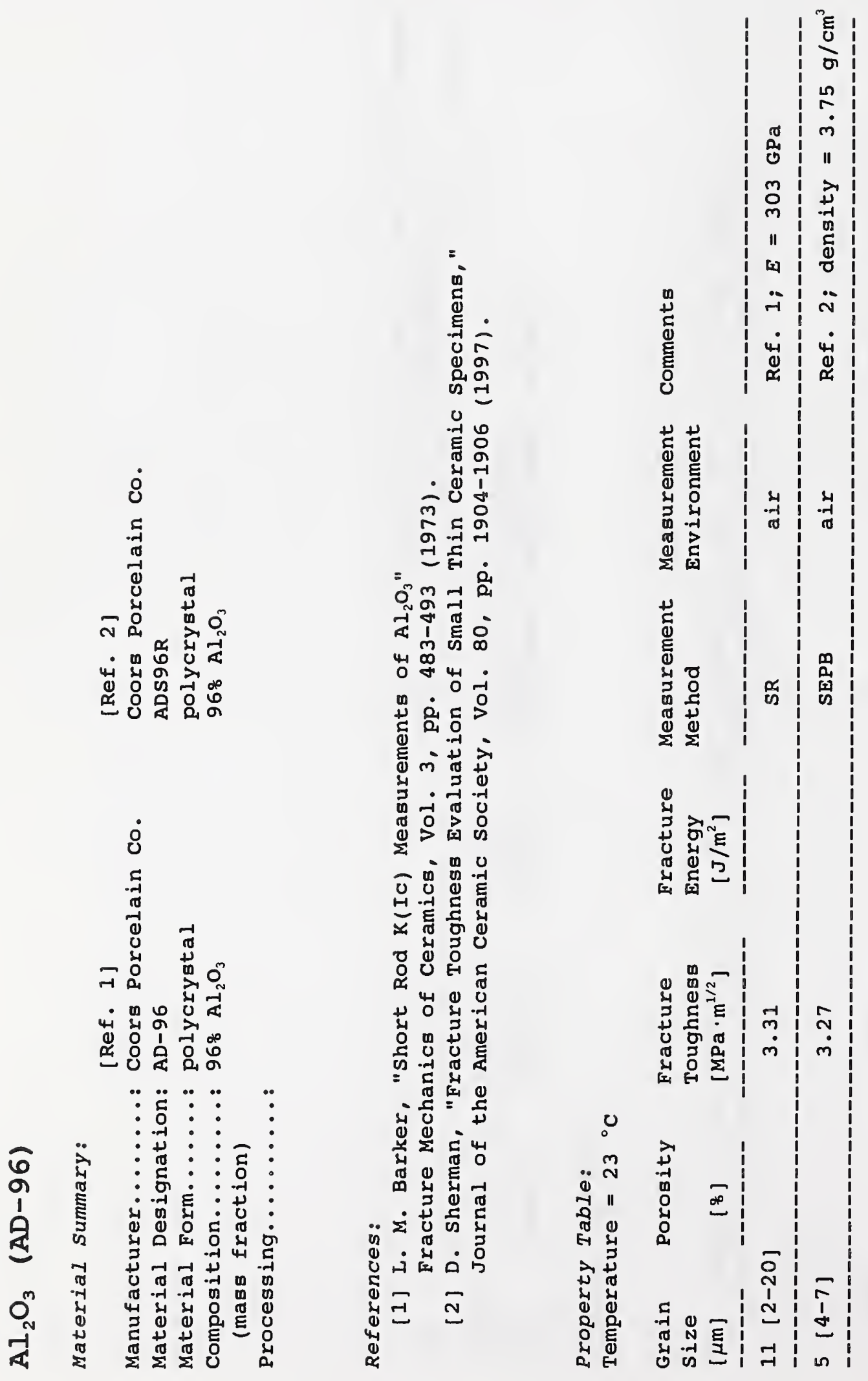

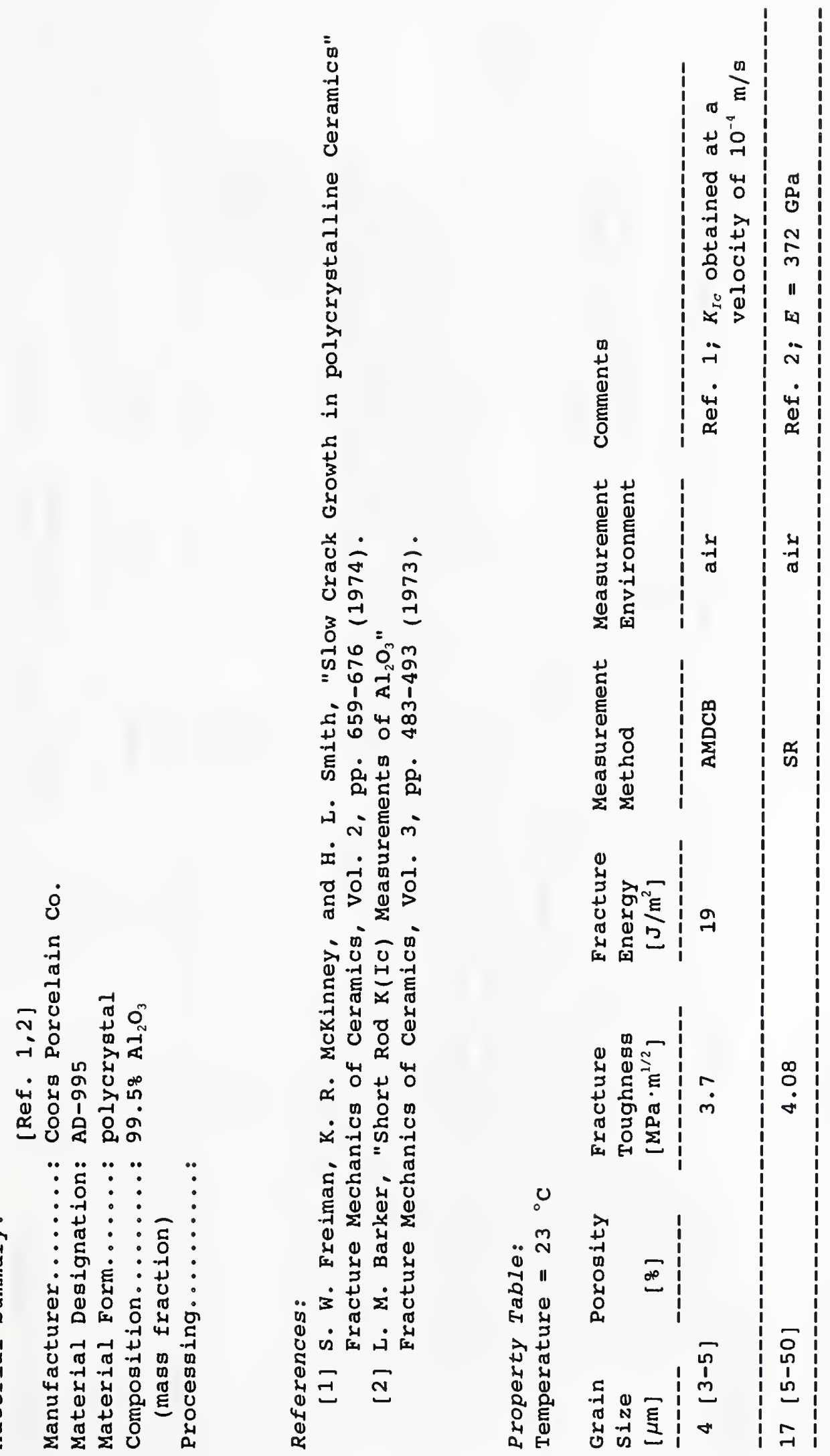

$\stackrel{1}{1}$ 

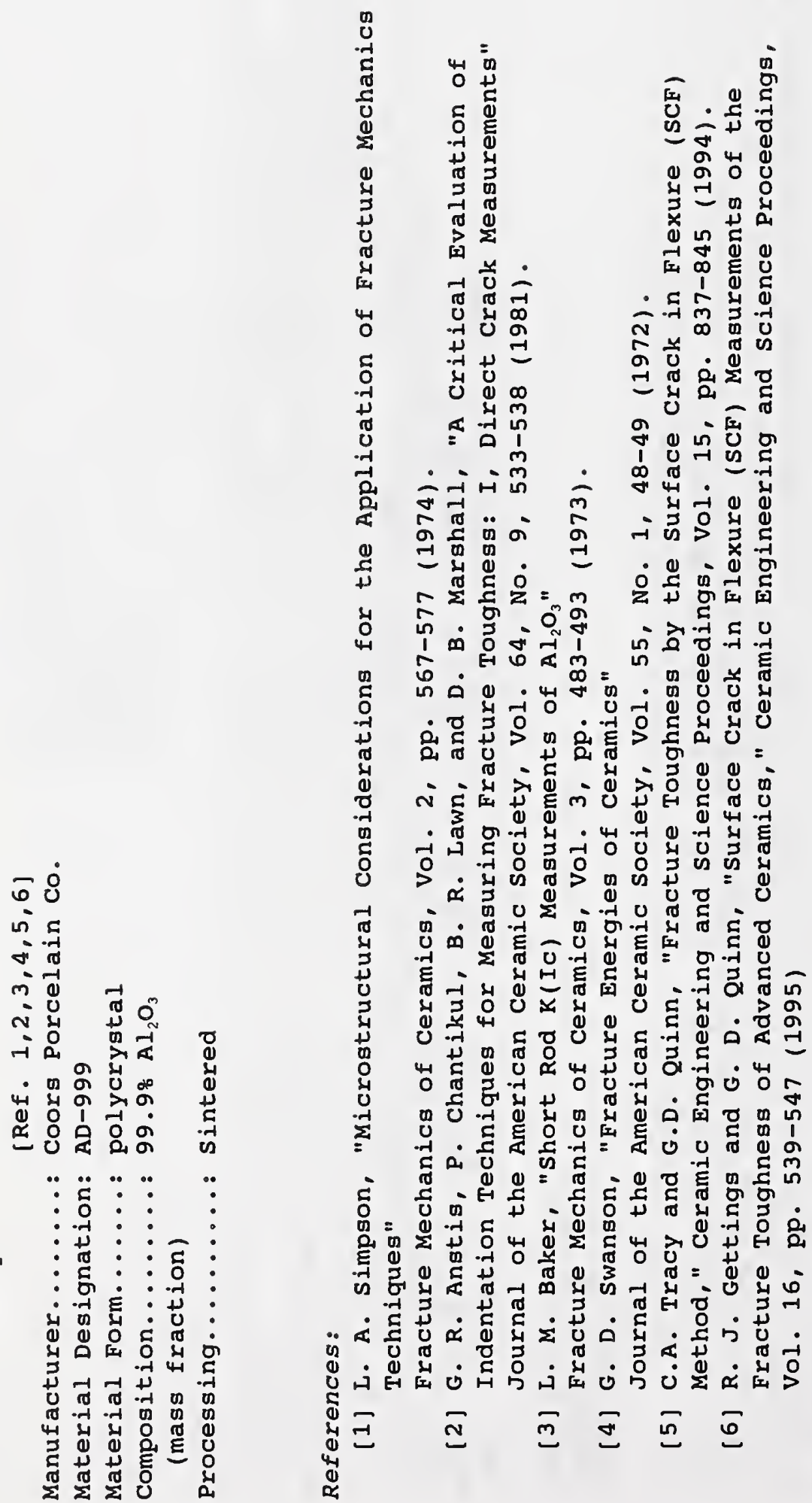


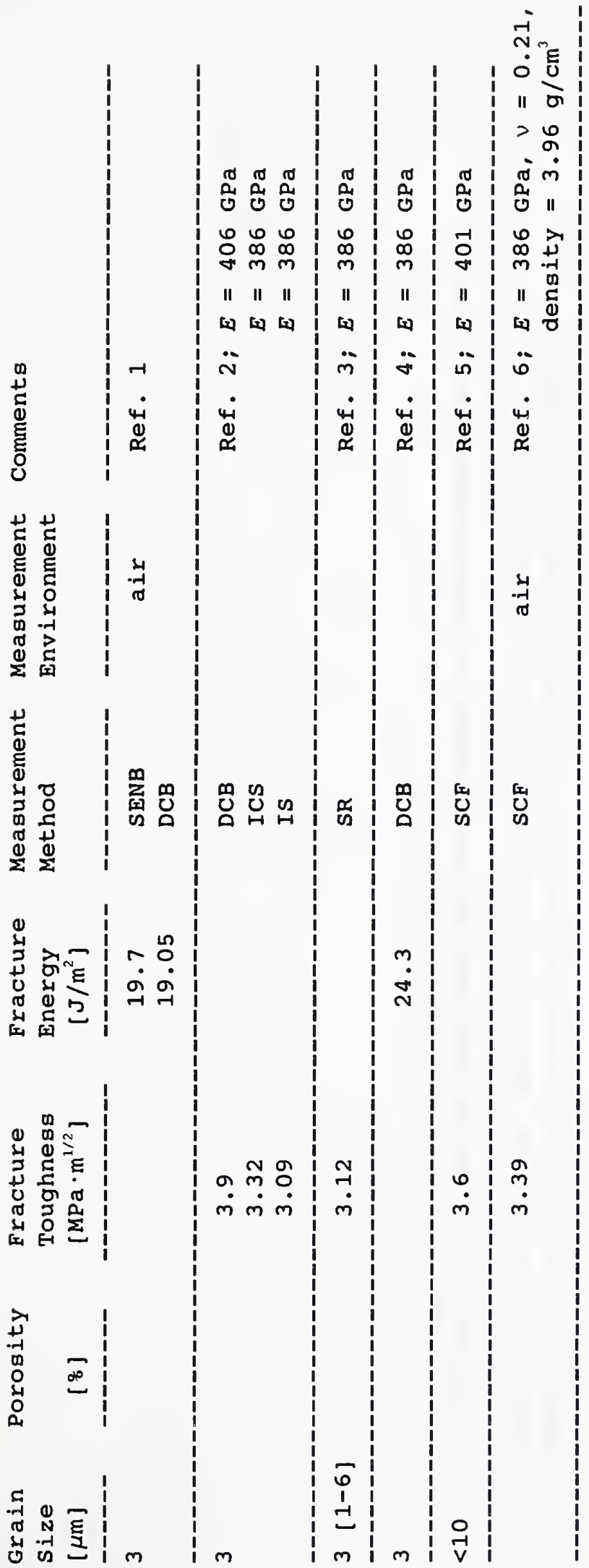




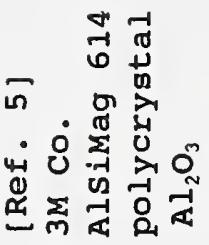
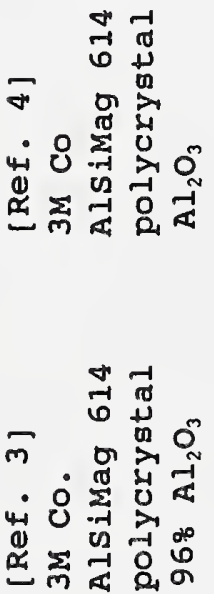

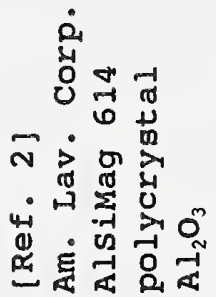

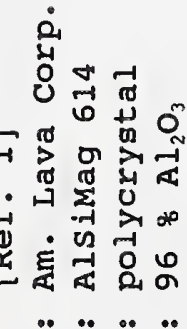

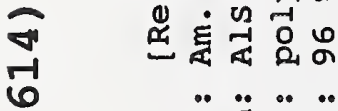

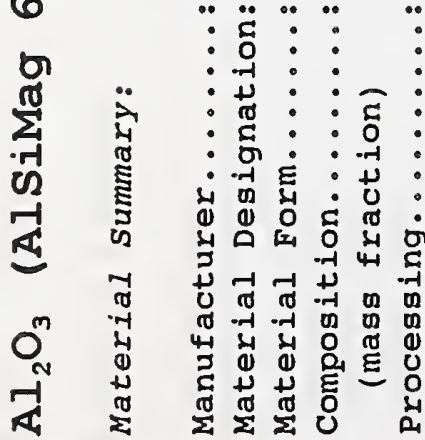

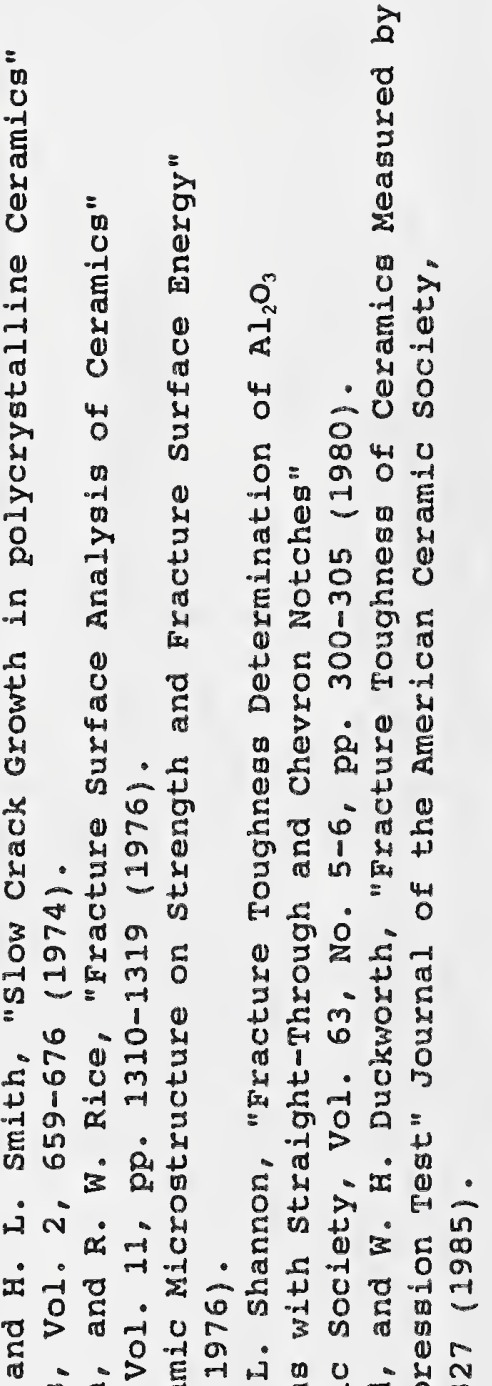

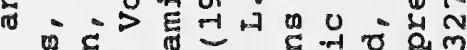
خ ह

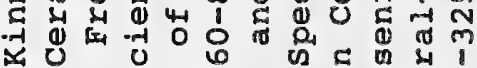

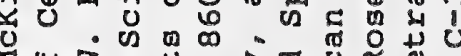

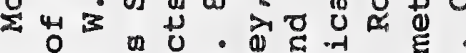

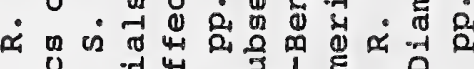
年

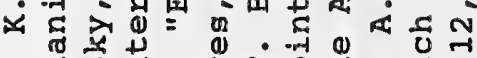

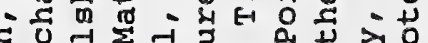
द्व $0 \begin{aligned} & 0 \\ & 0\end{aligned}$

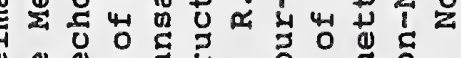

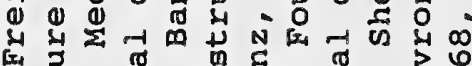
[ iิ $\dot{0} 0$ 出

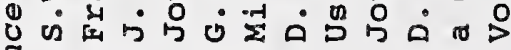
离 


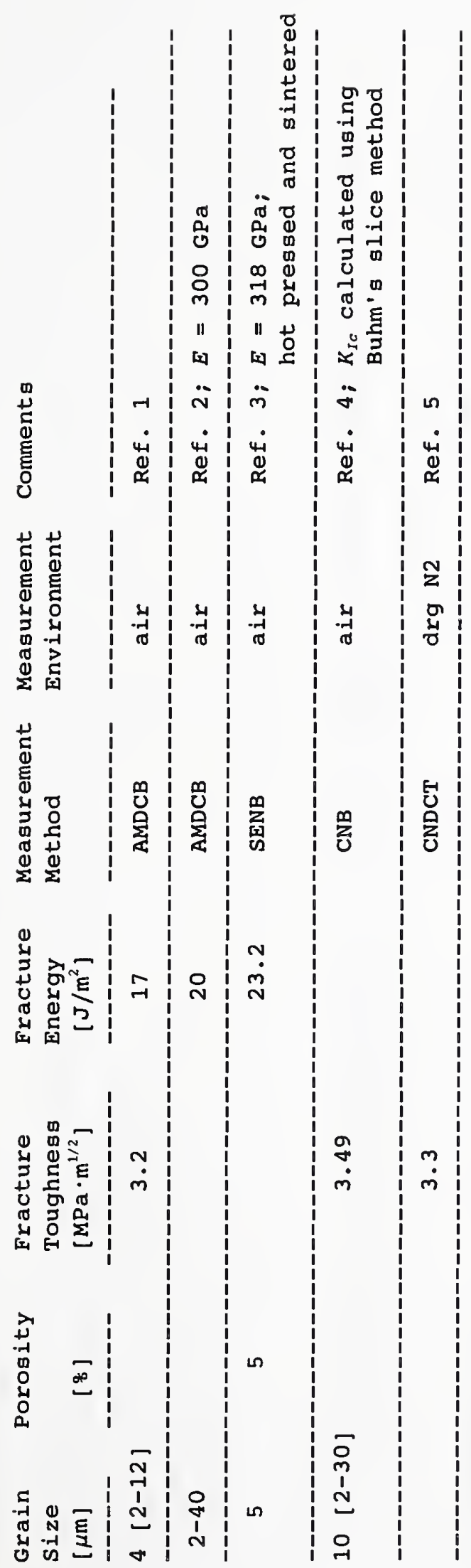




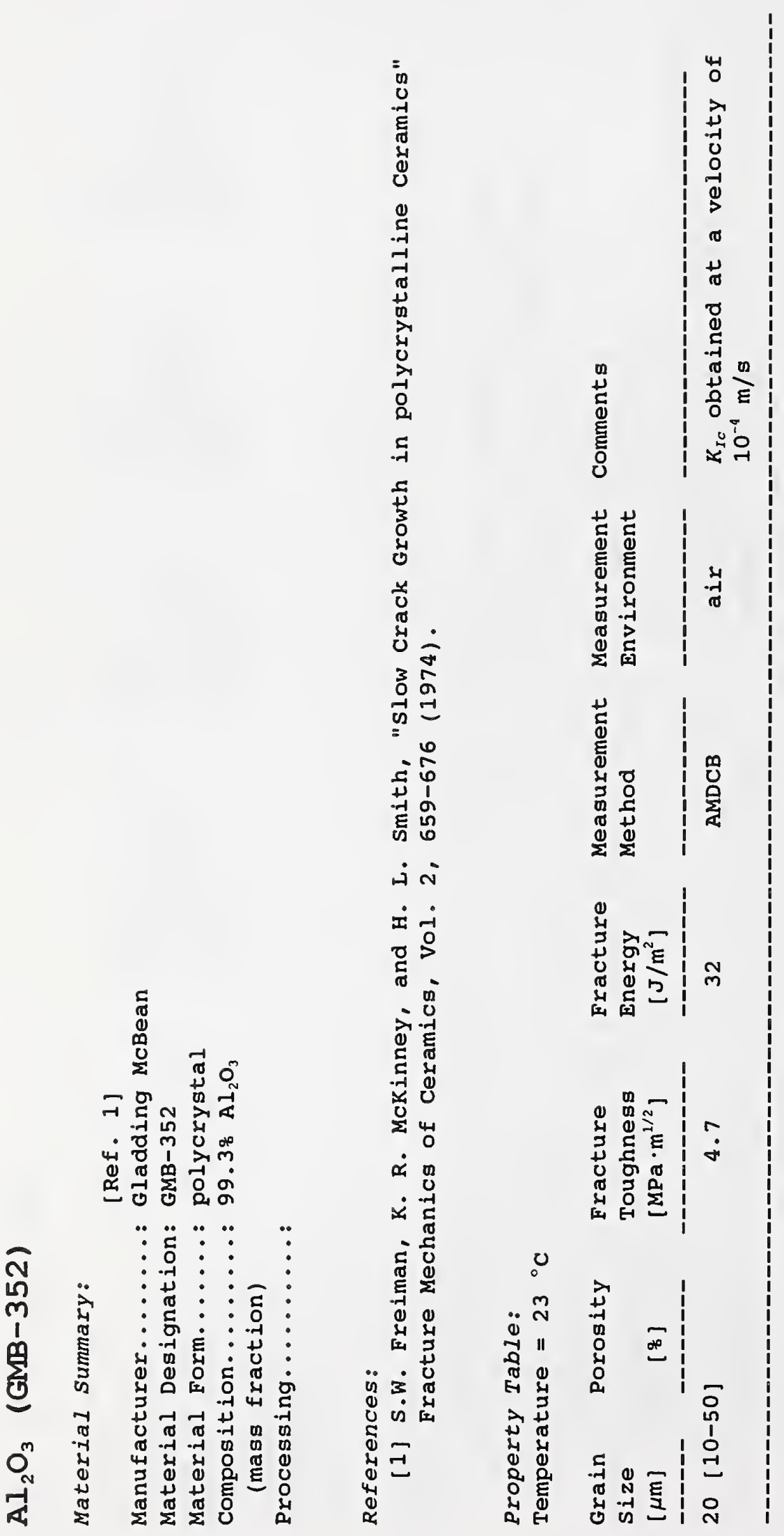




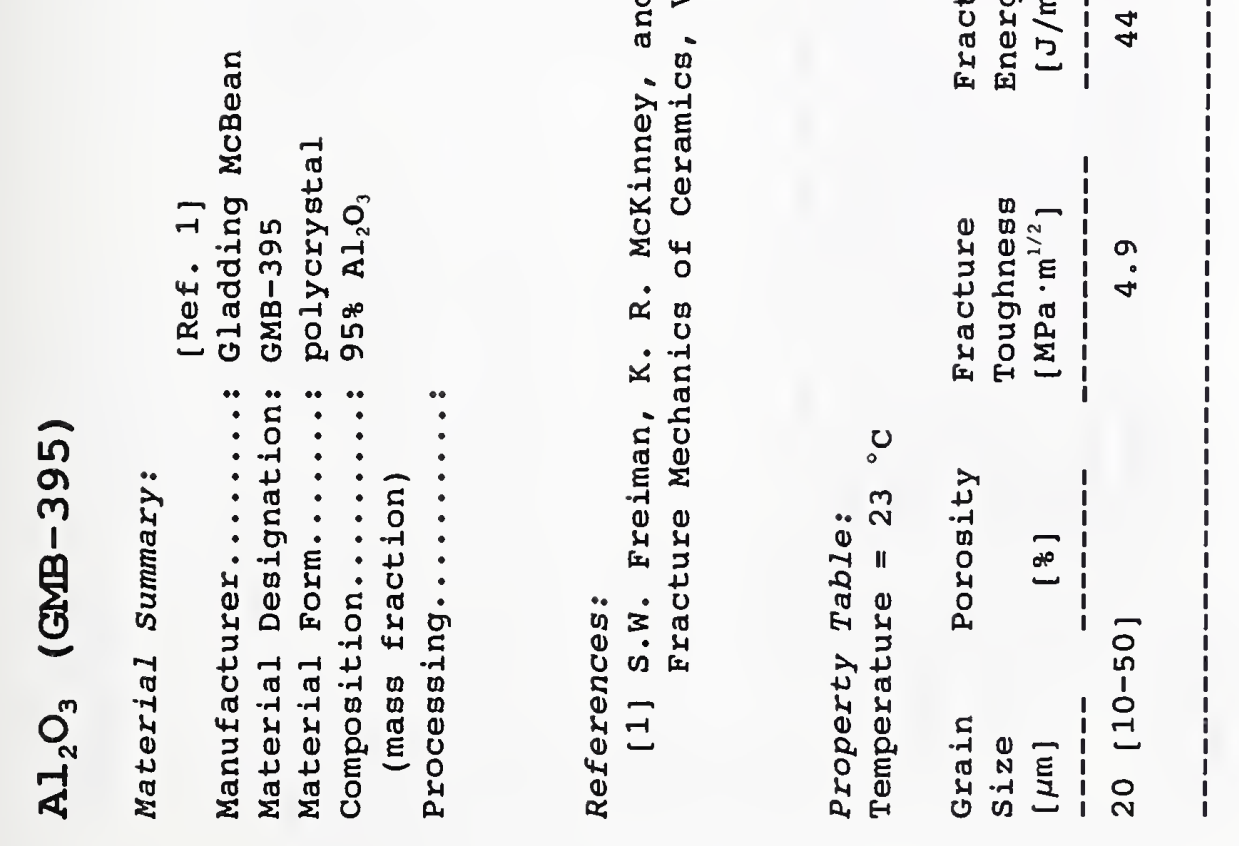



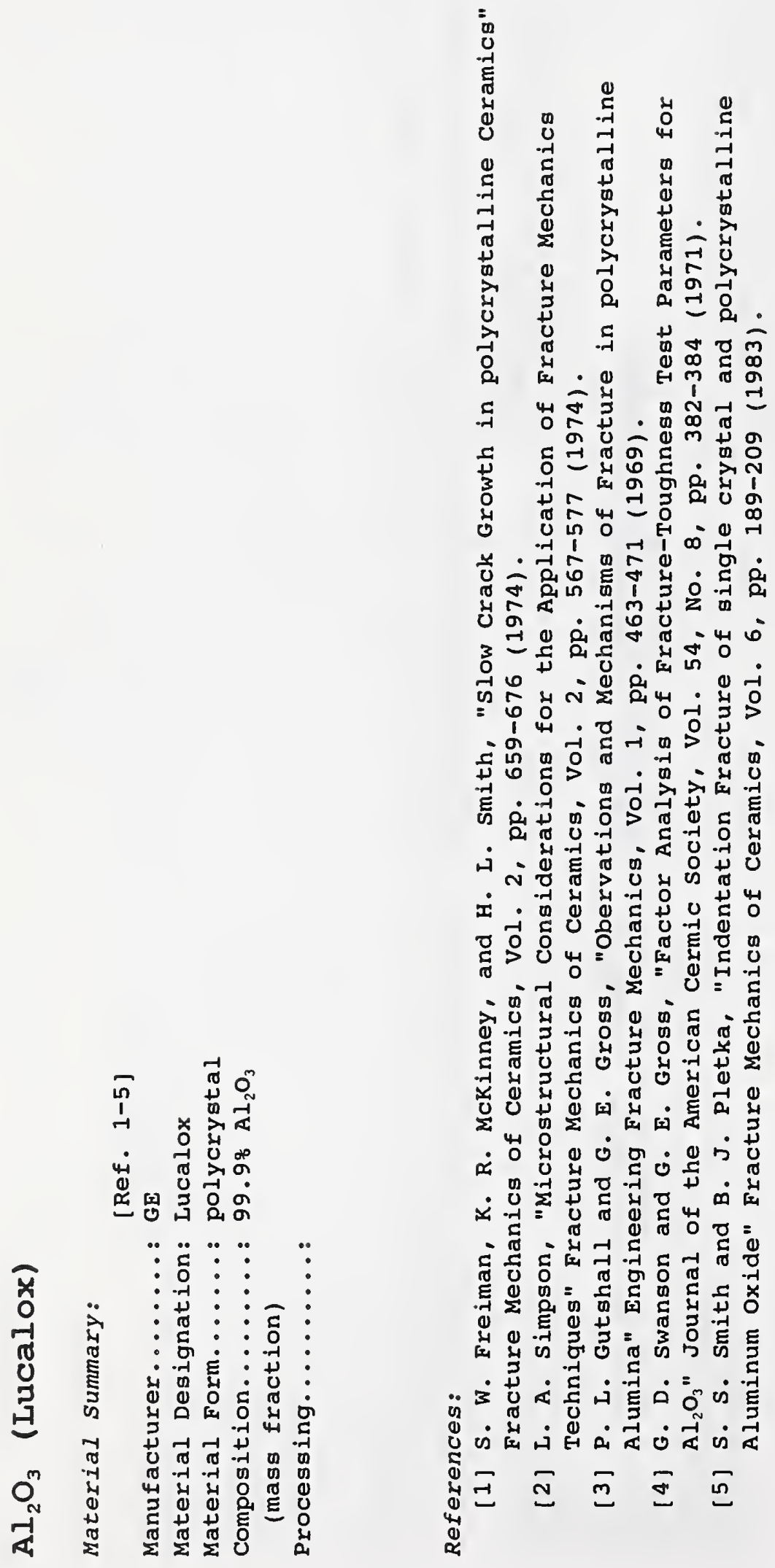


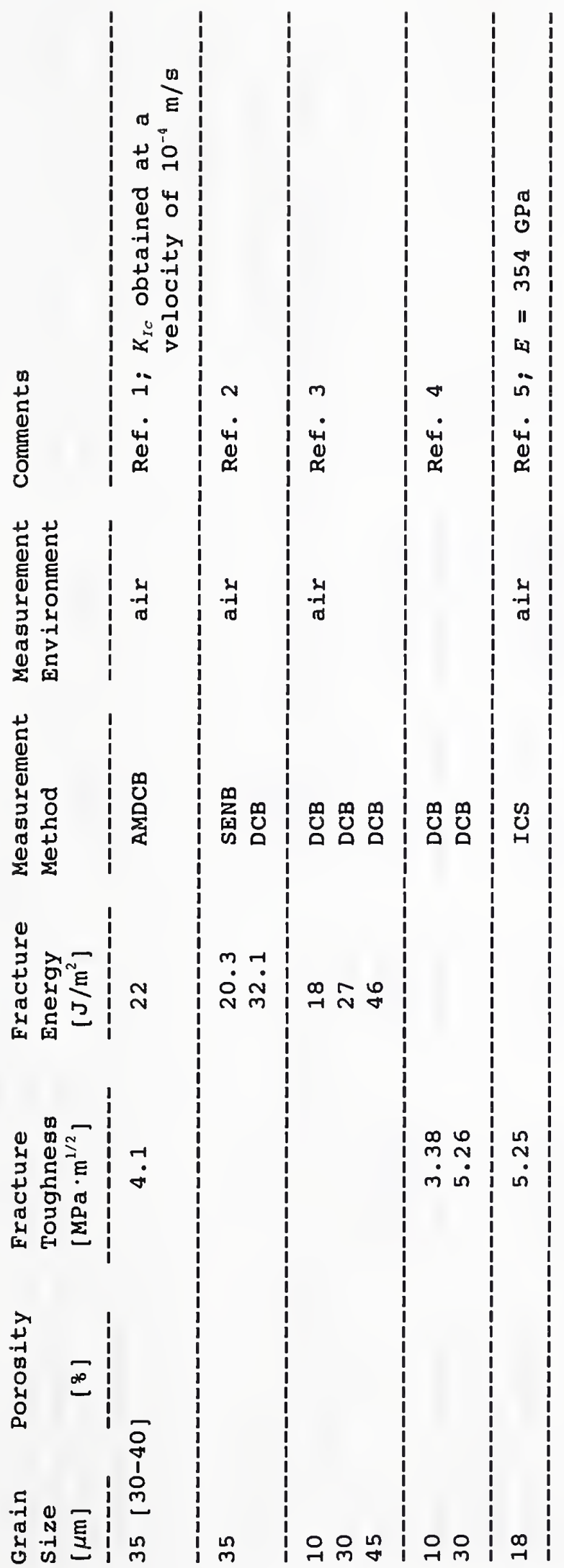




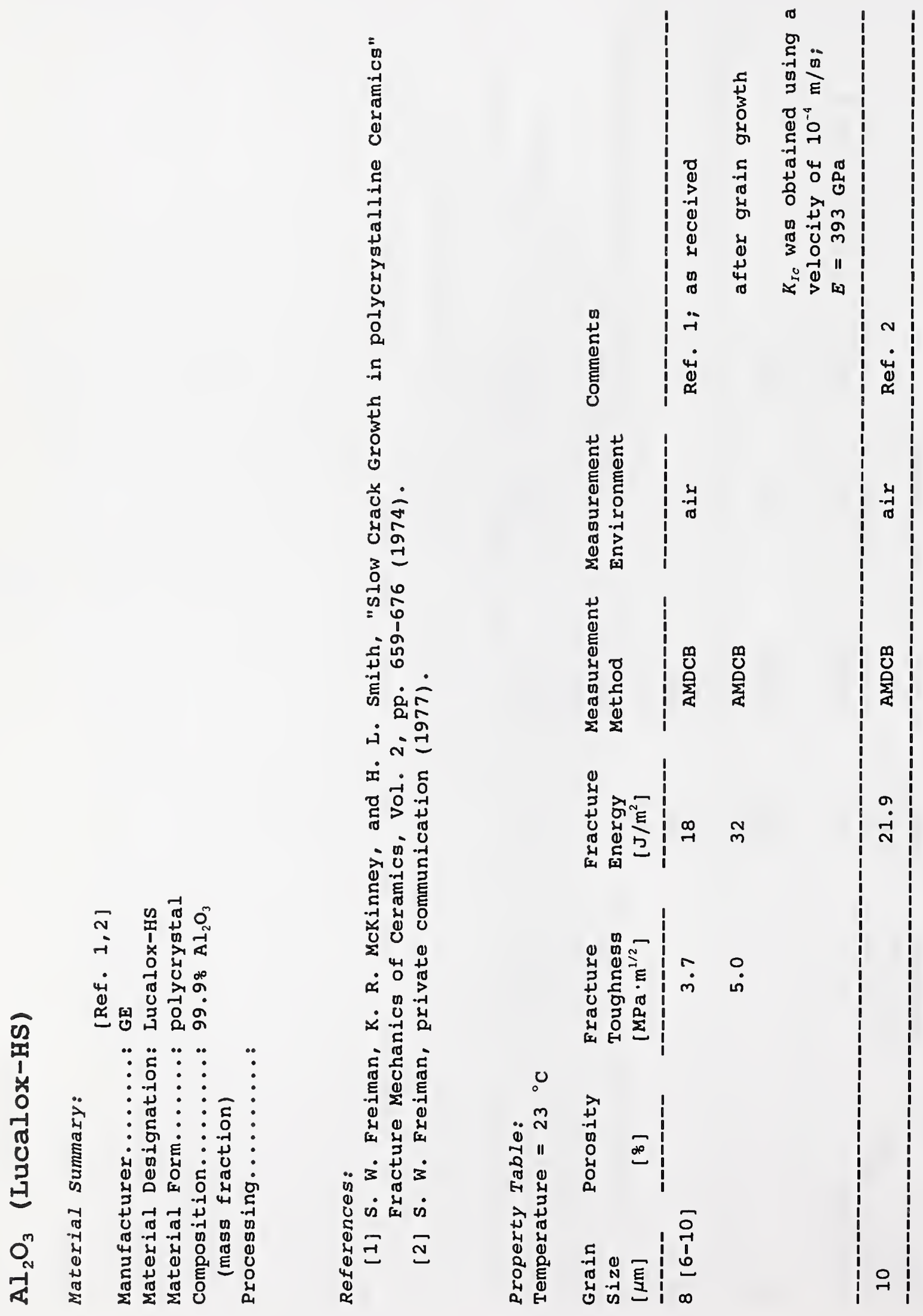




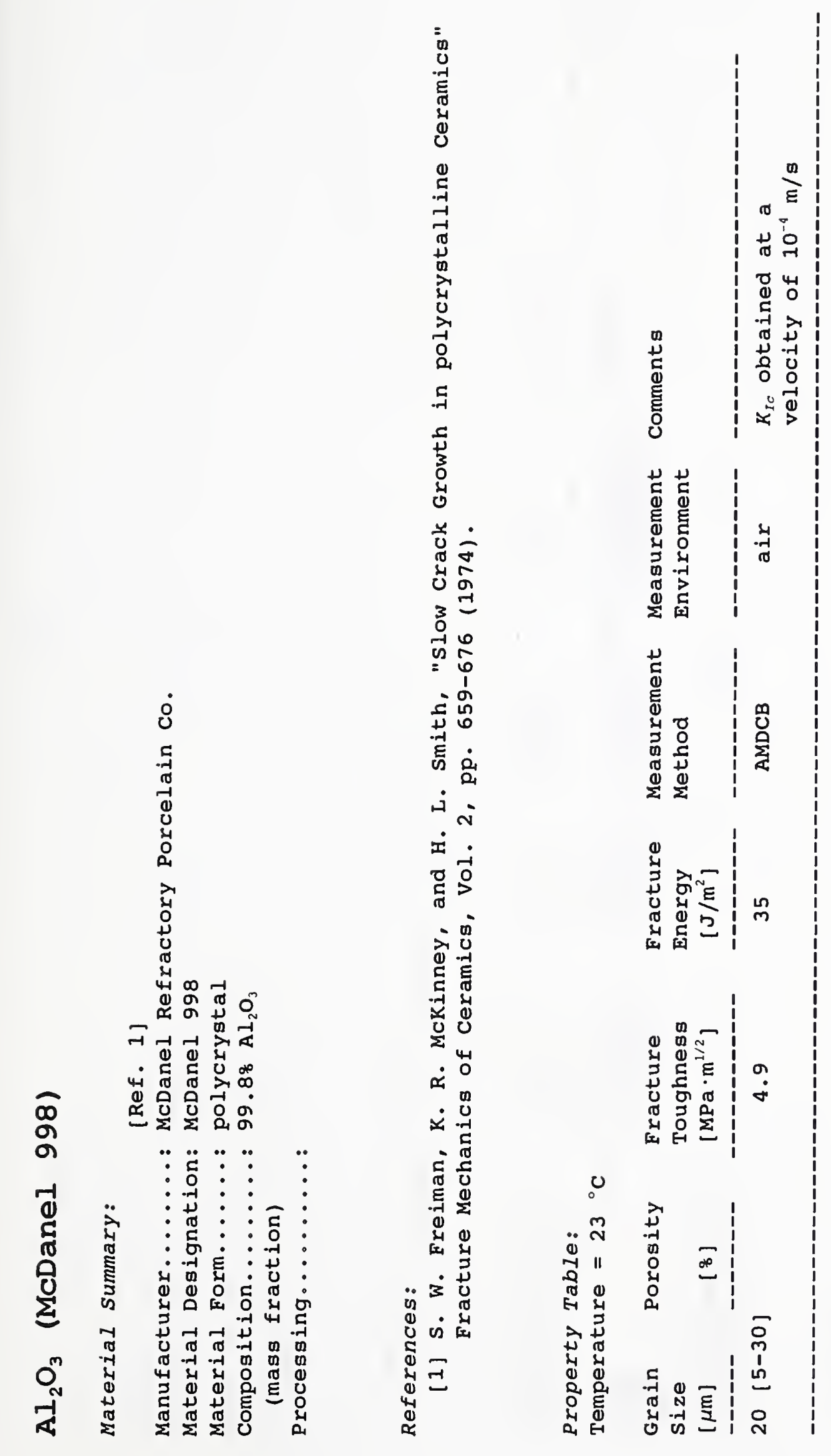



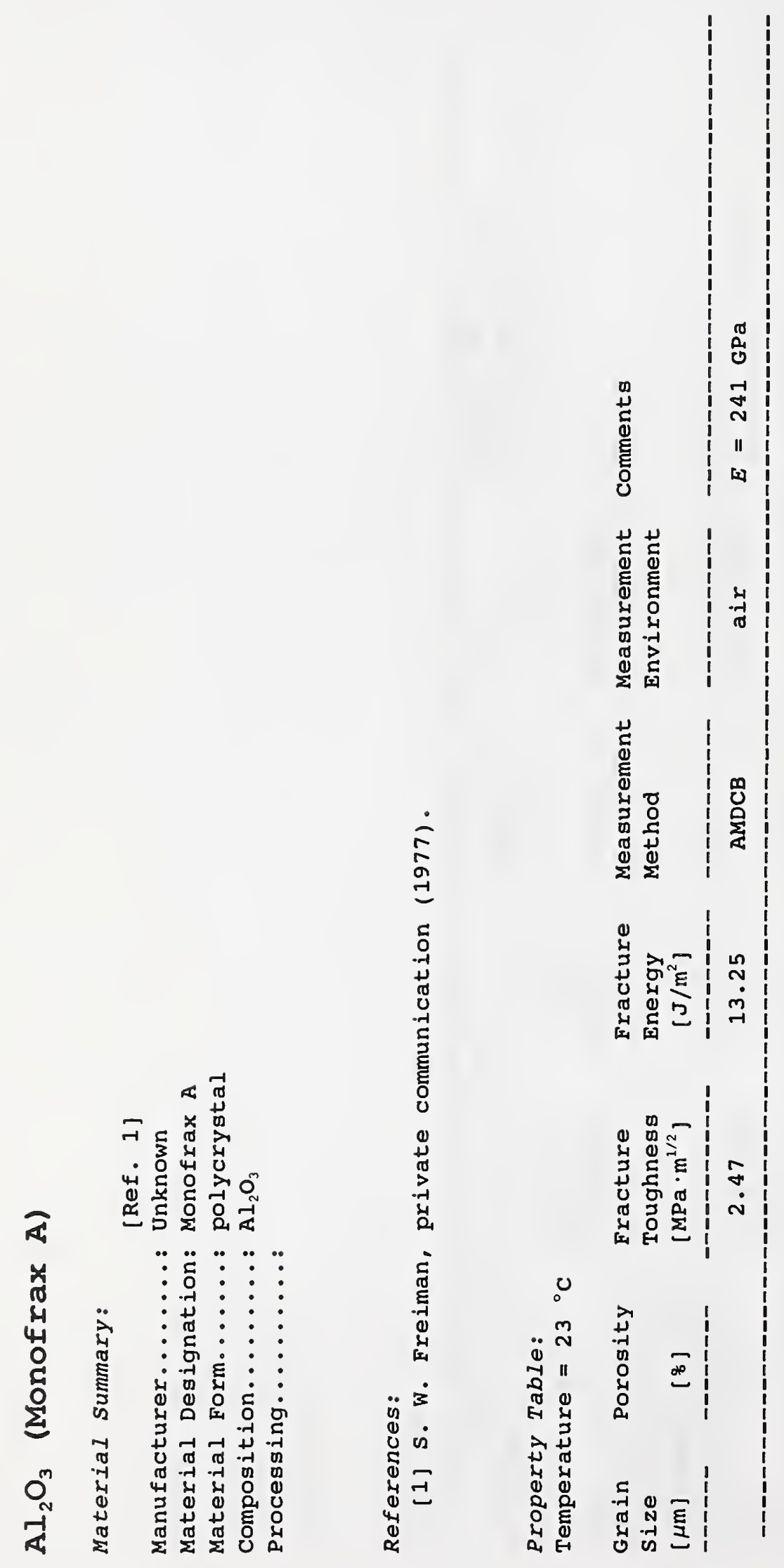


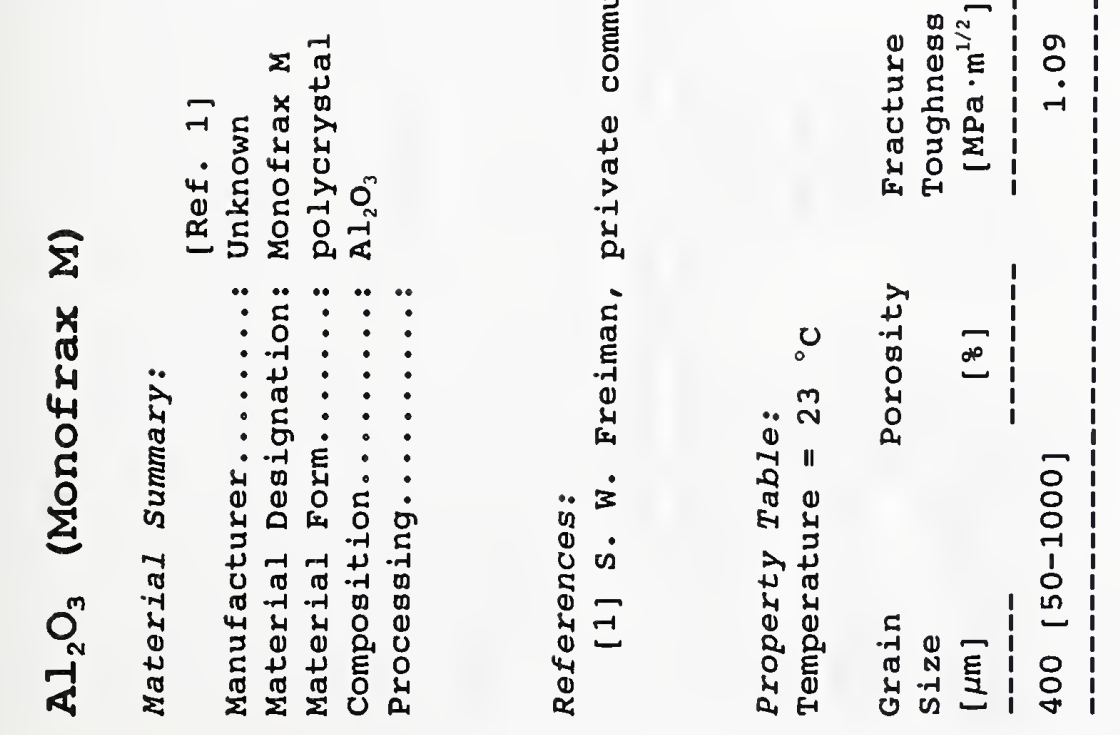



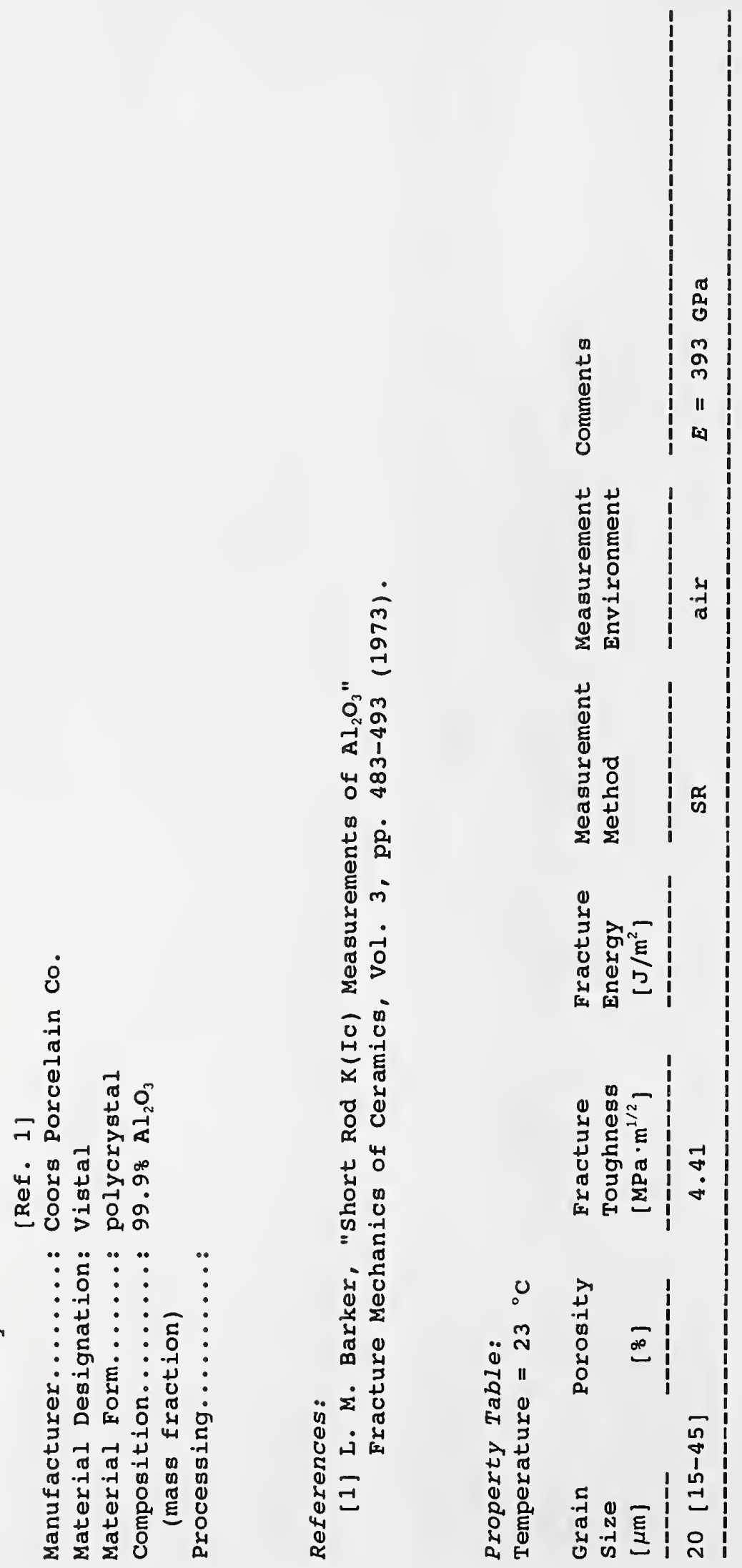


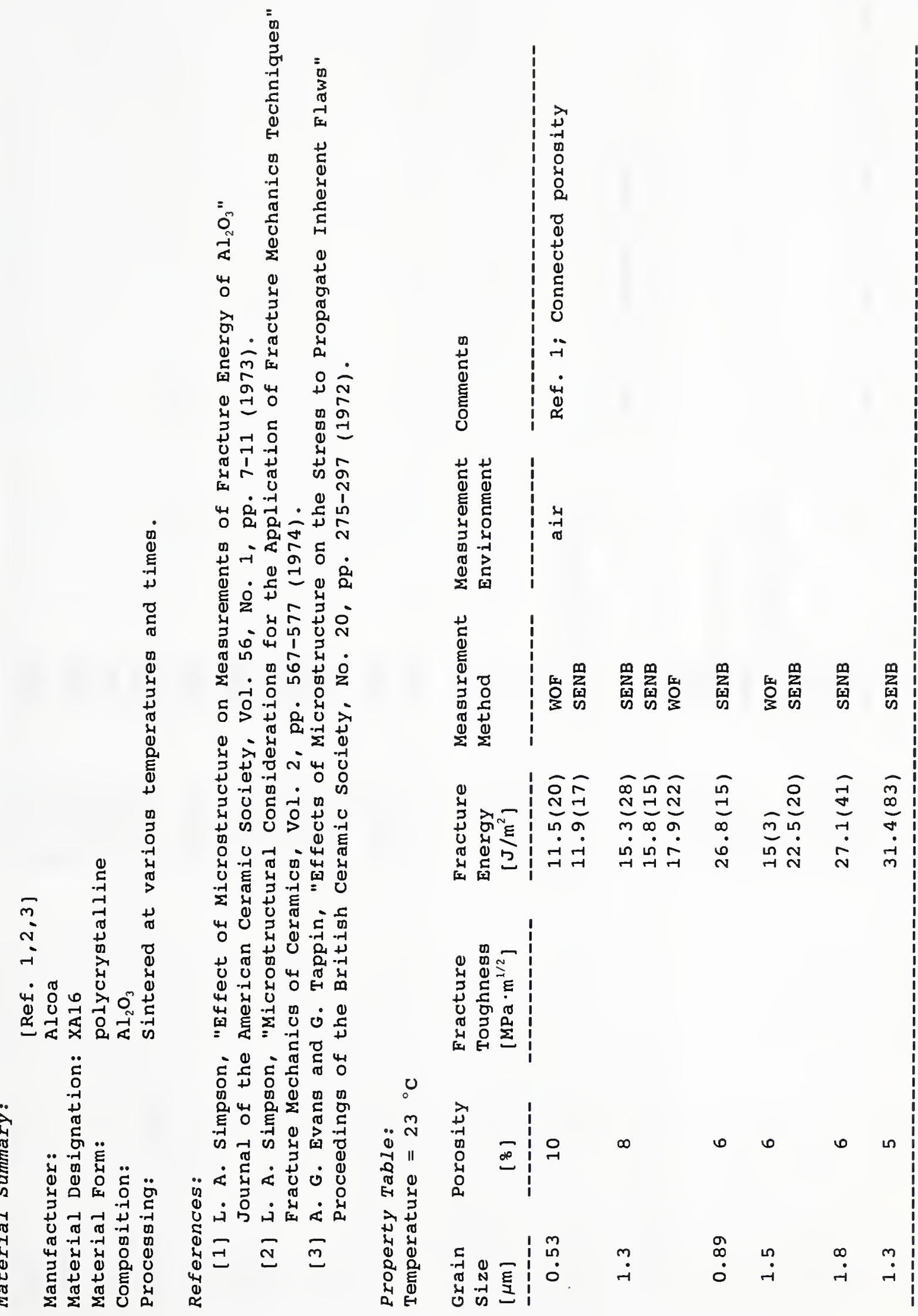



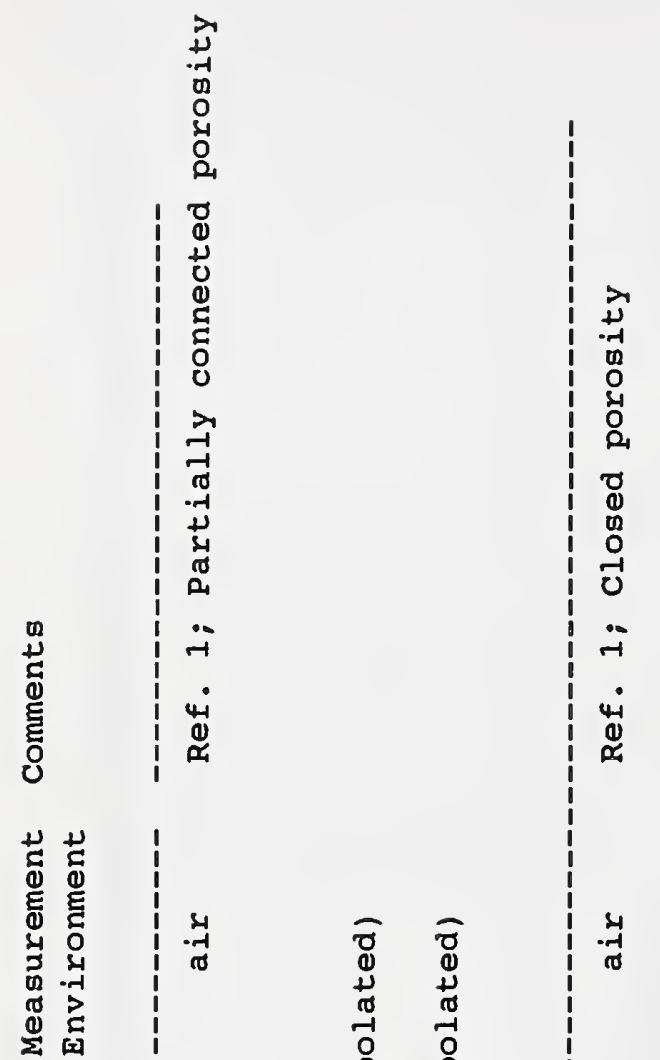

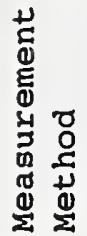
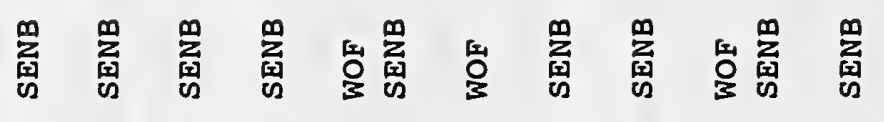

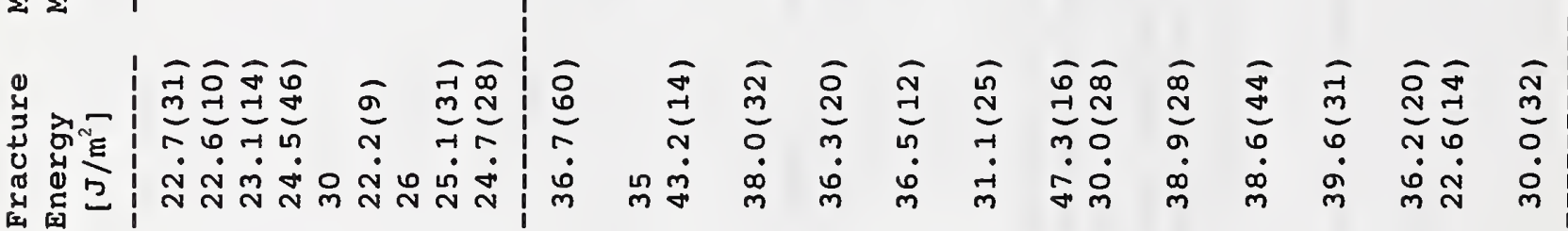

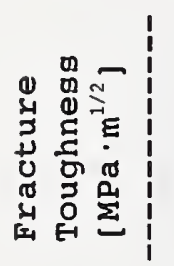

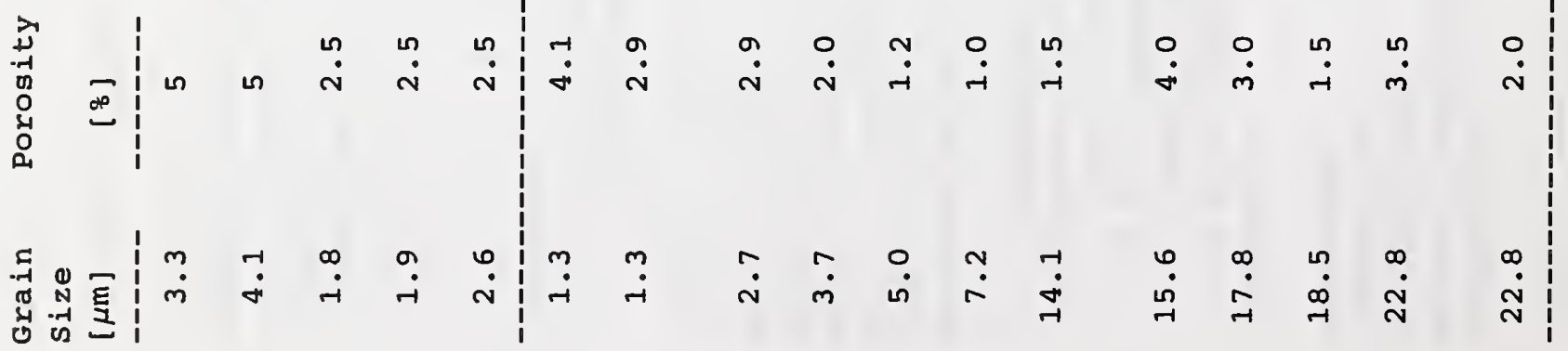




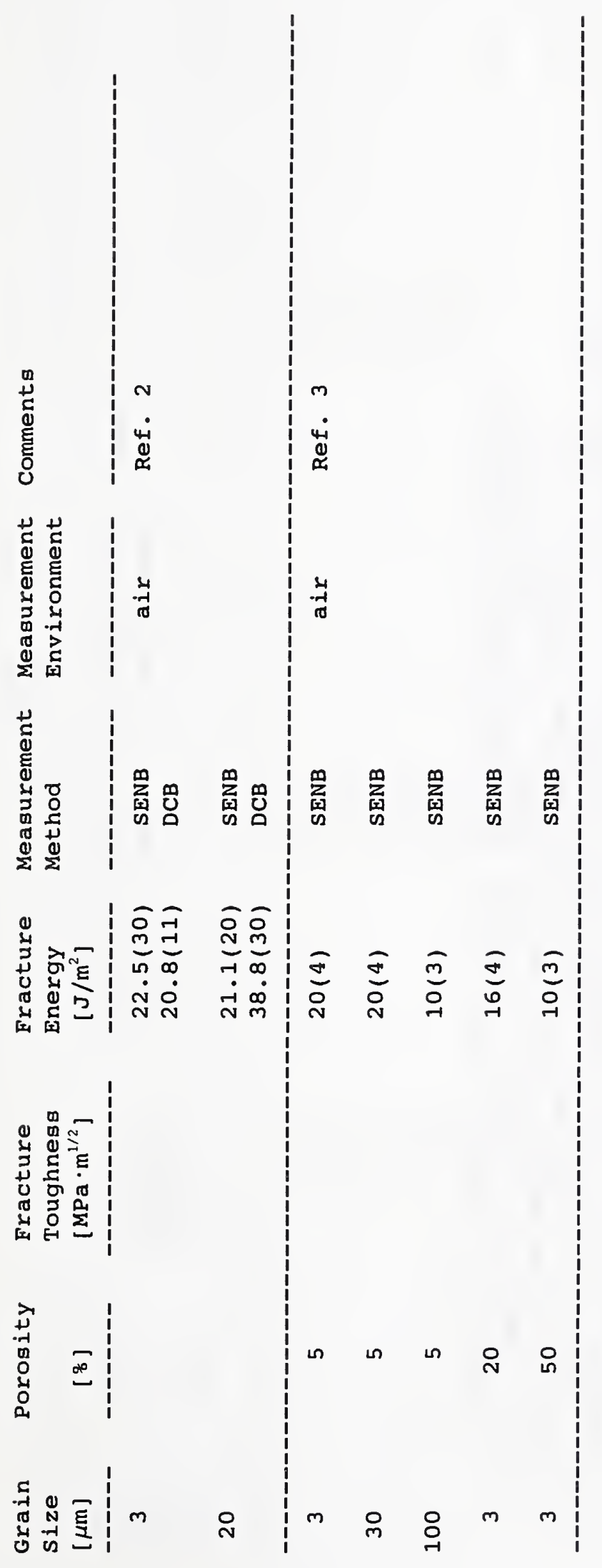



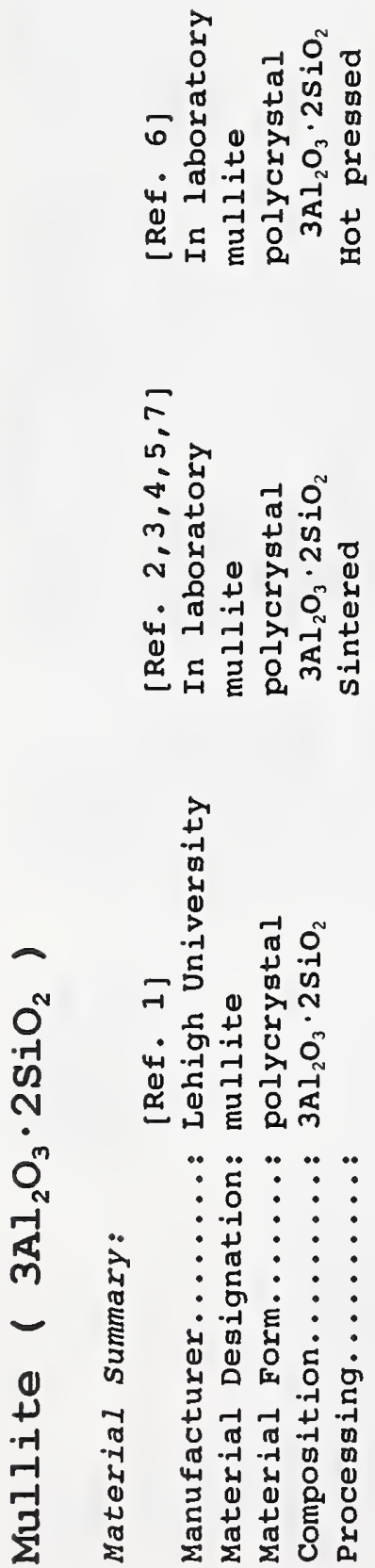

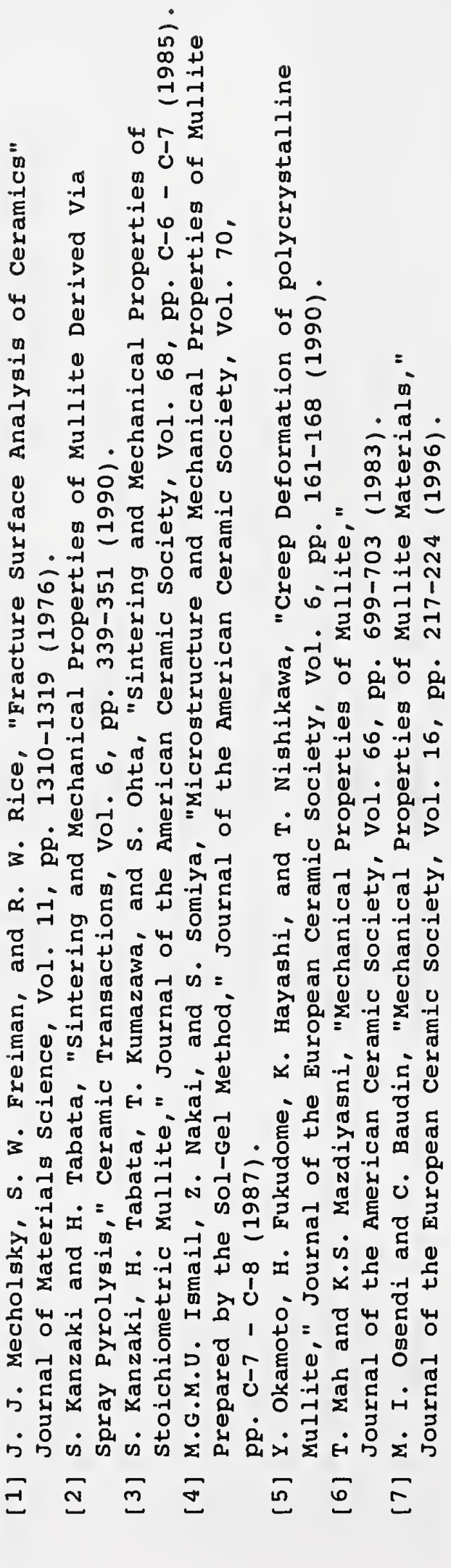




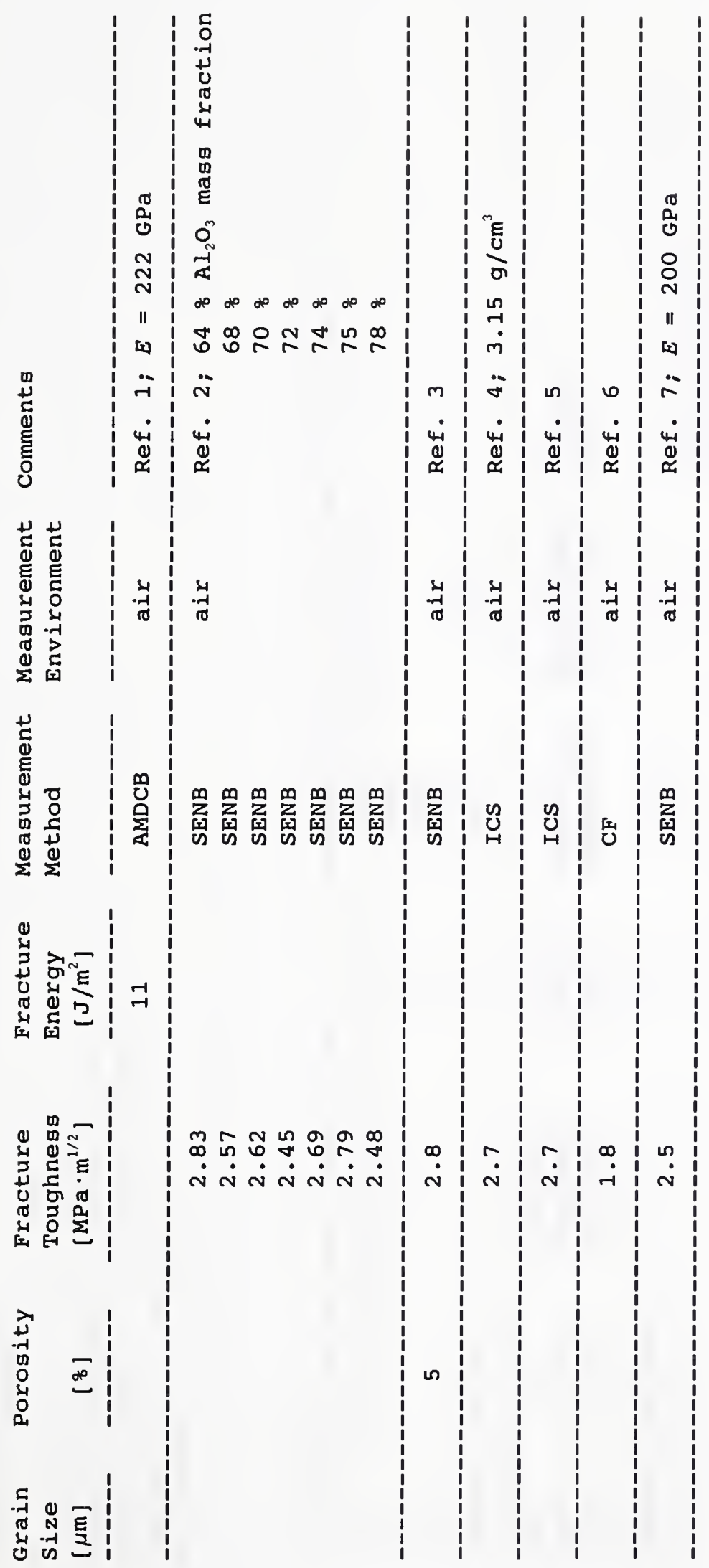




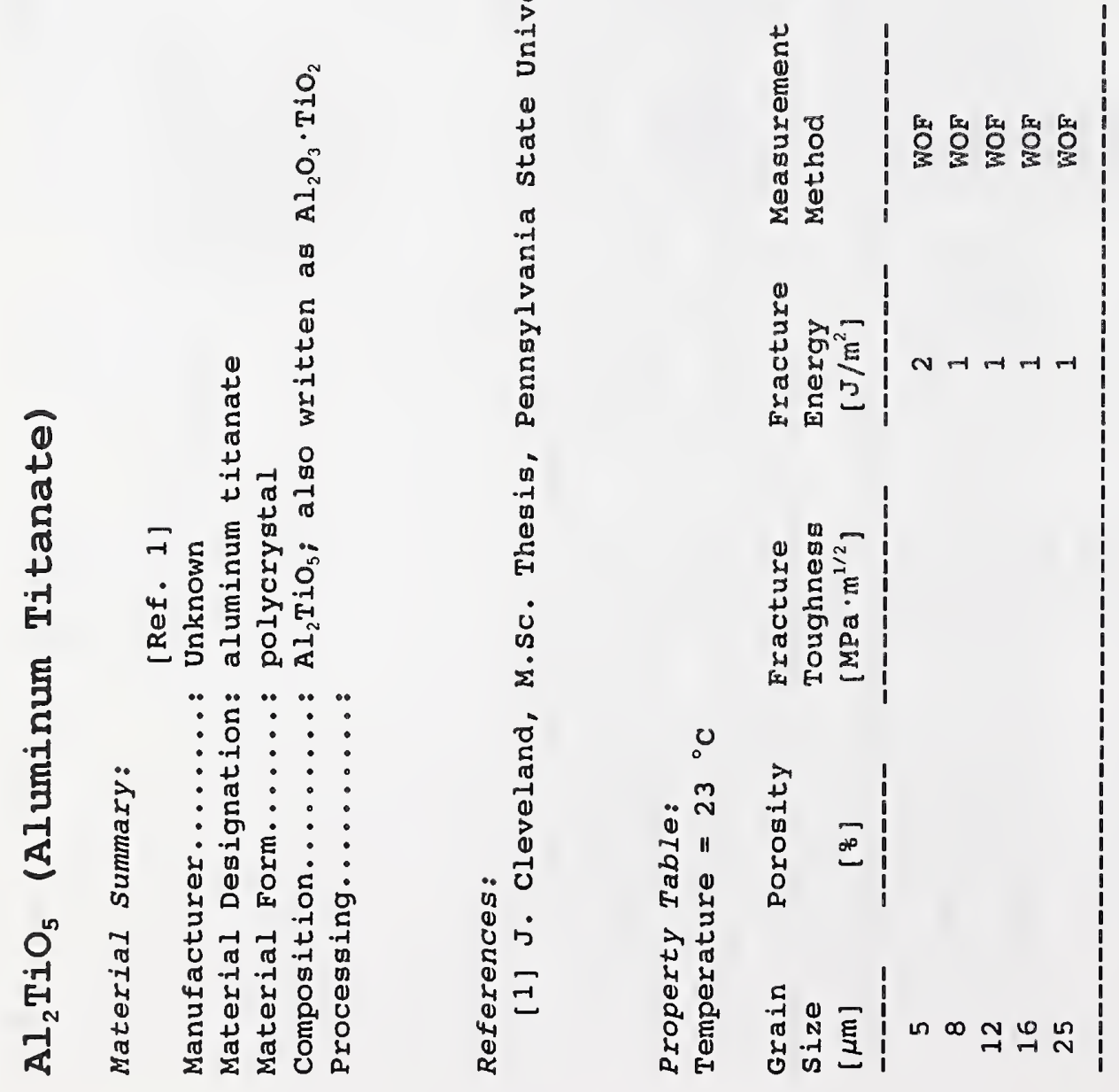




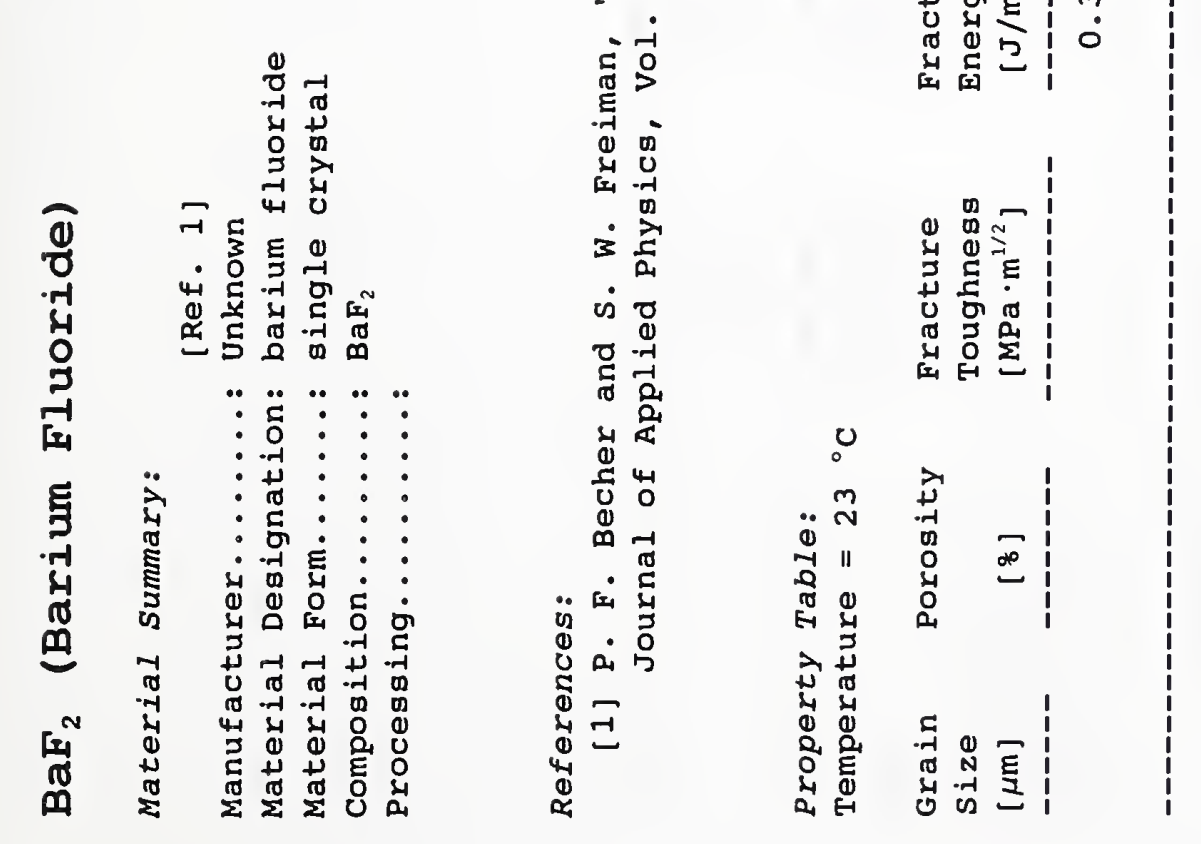




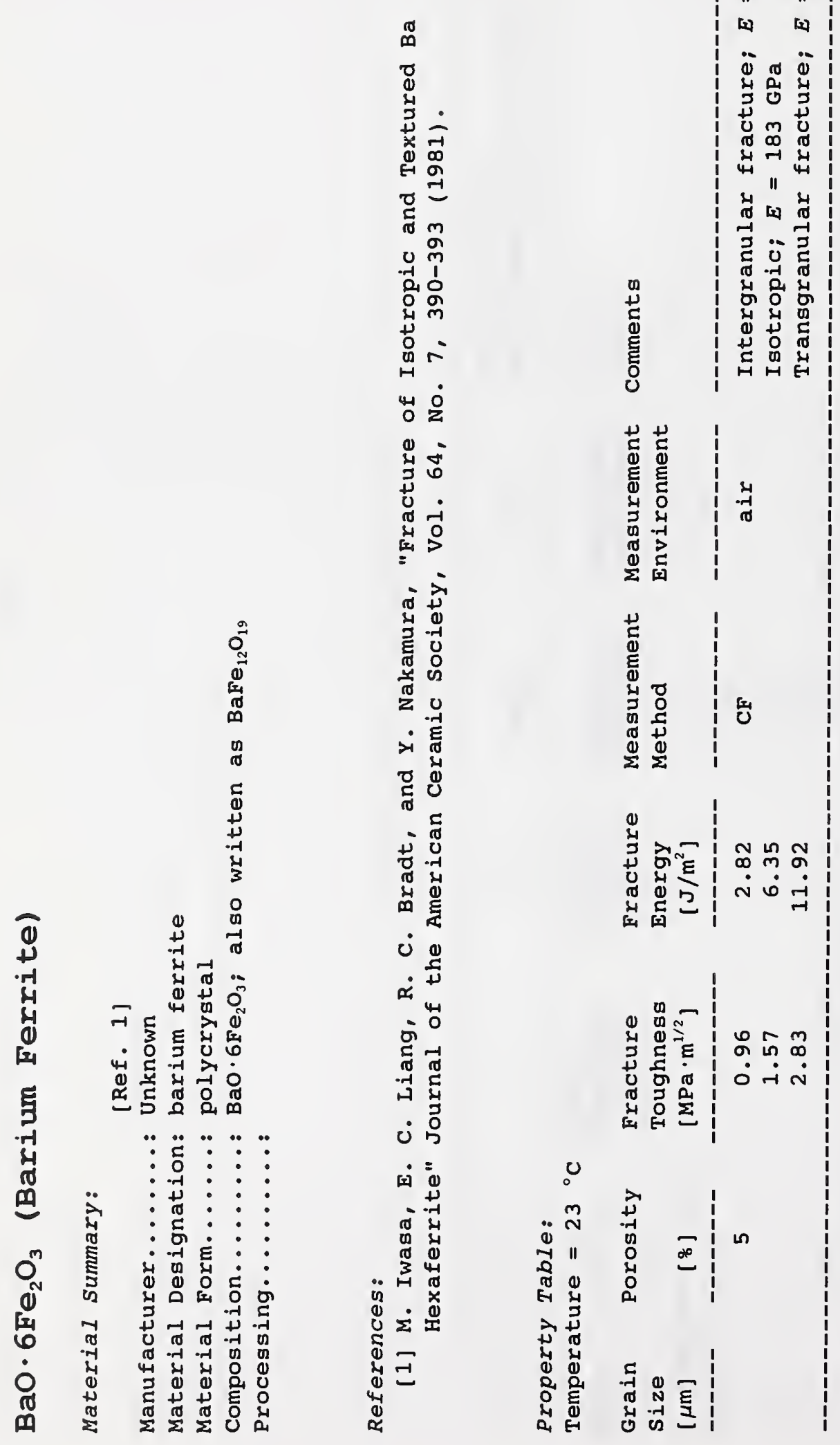




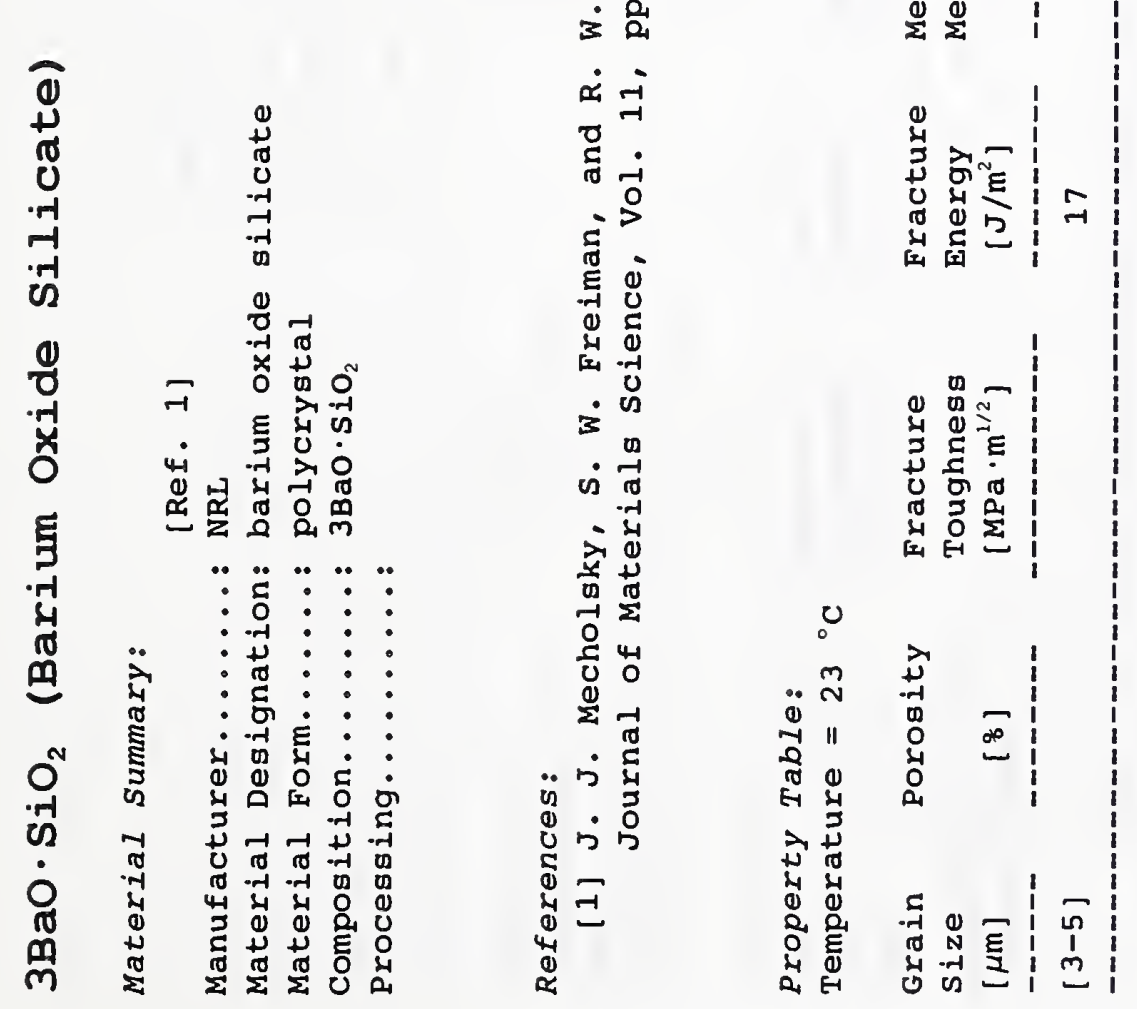




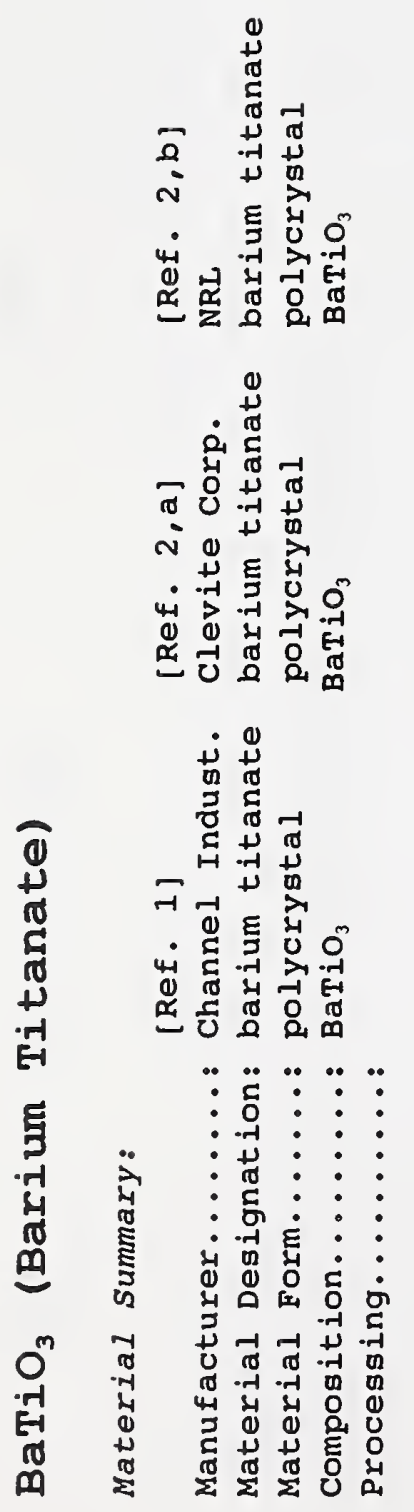

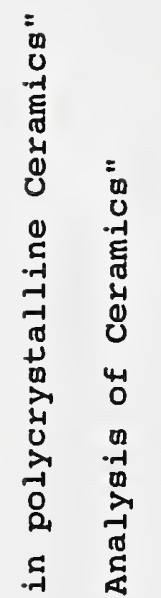

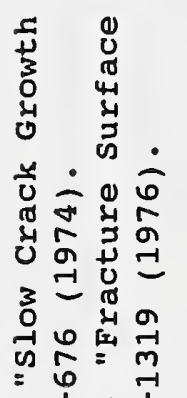

$=0$ 舀 步 0 年 岳宫家宫 نं $\dot{0}$. 的 ช 市政 入े ह है

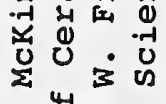
வ ${ }_{\infty}^{4}$ के

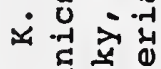

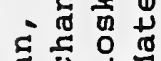
ฮี่ 寻文员㟧 出望些 $\therefore$ 常 的点的号 离

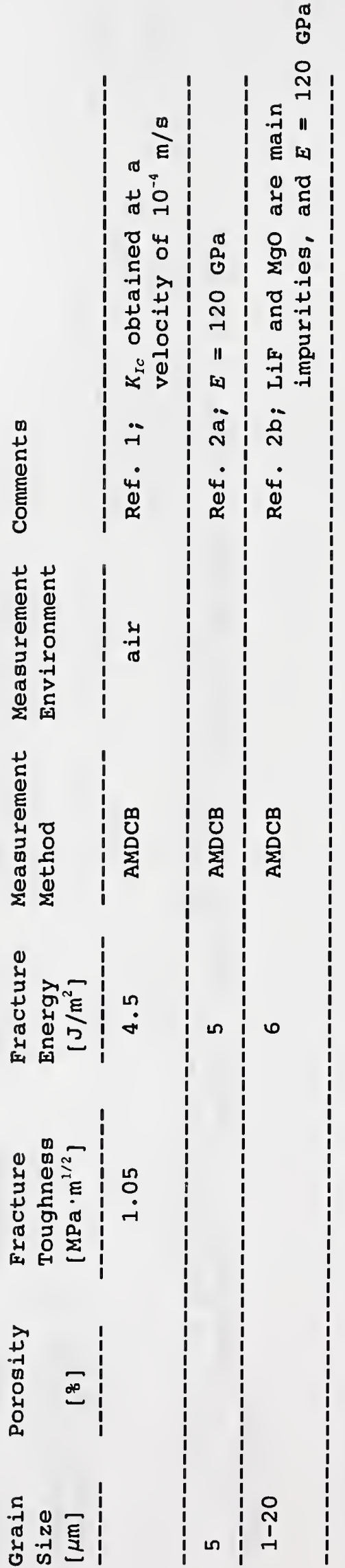



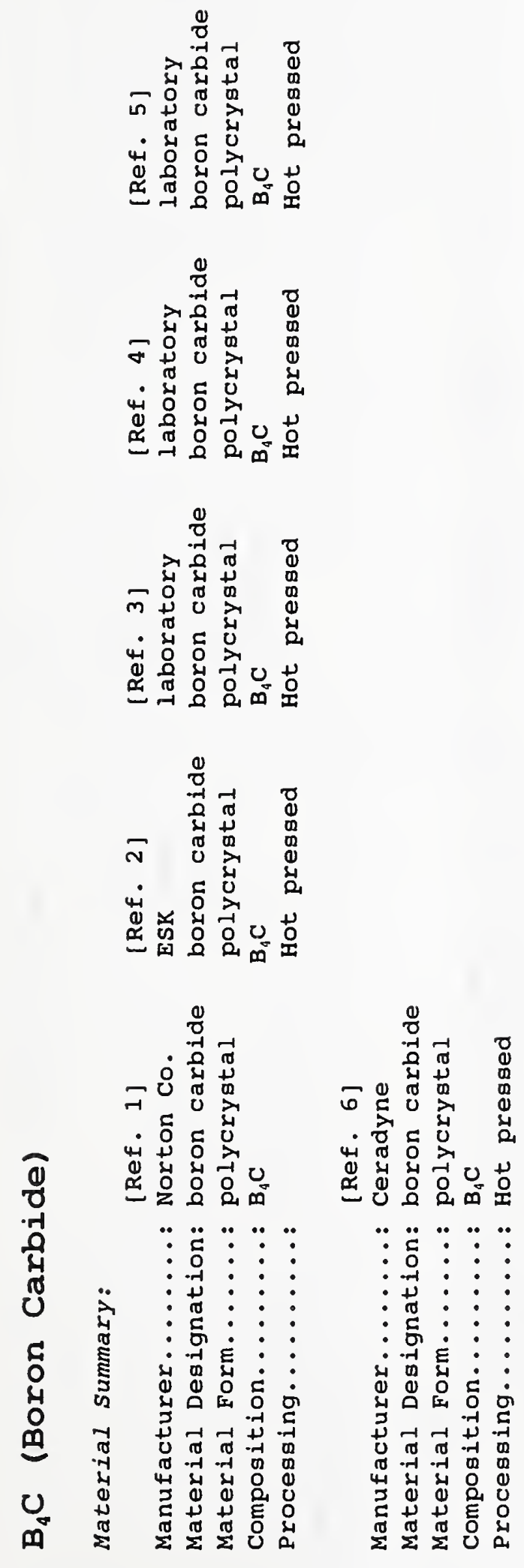

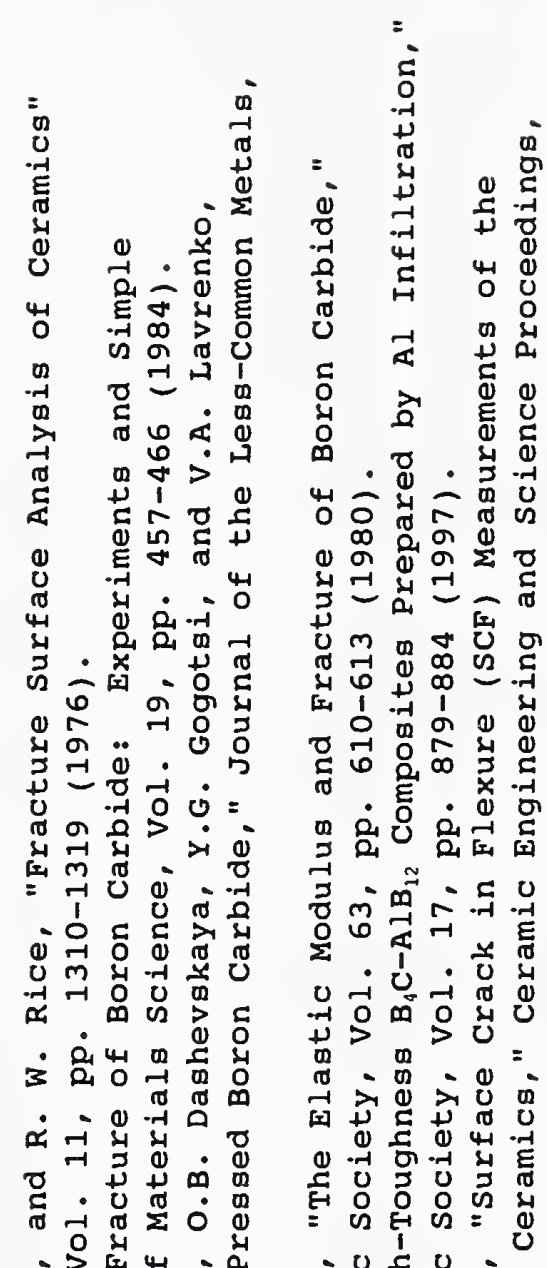

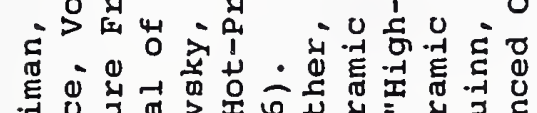

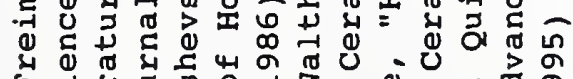

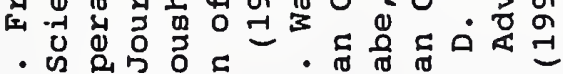

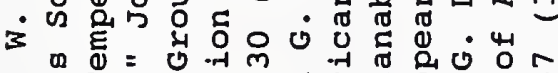
कि

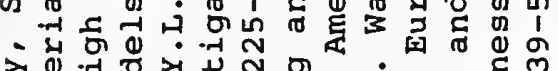

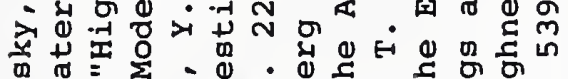

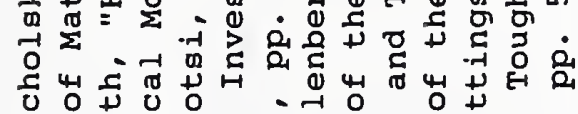

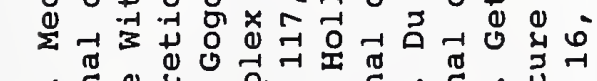

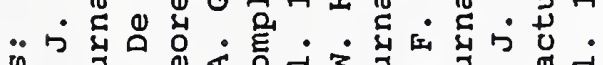

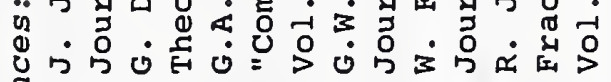

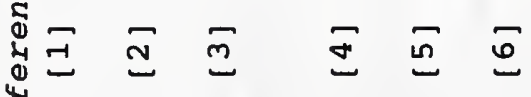




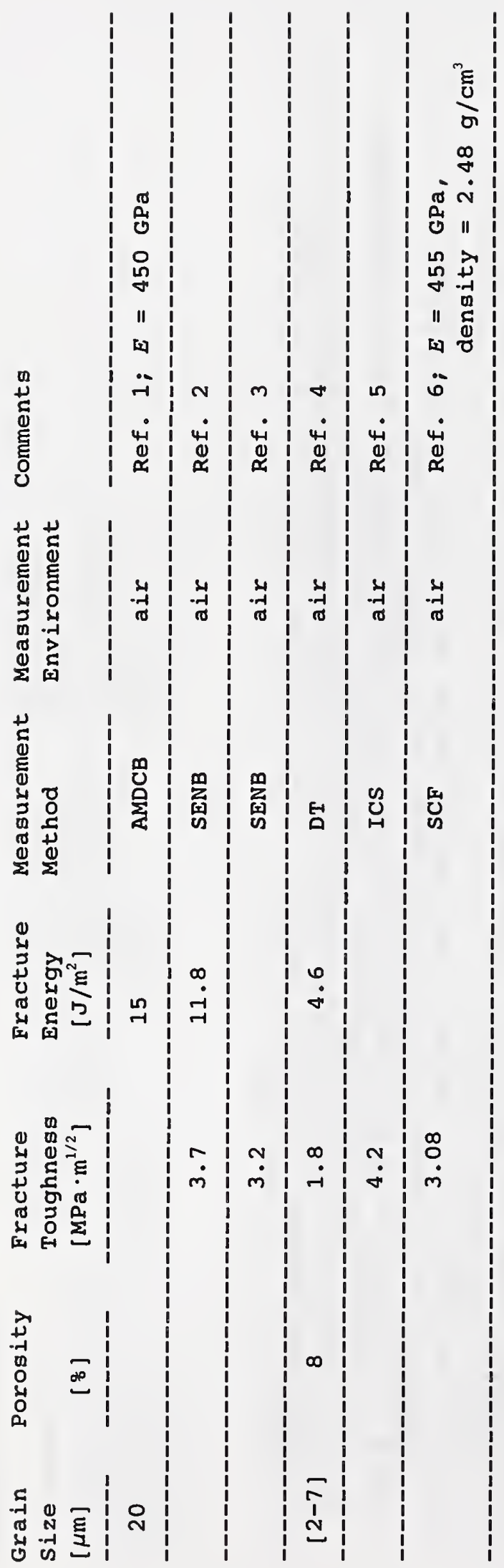



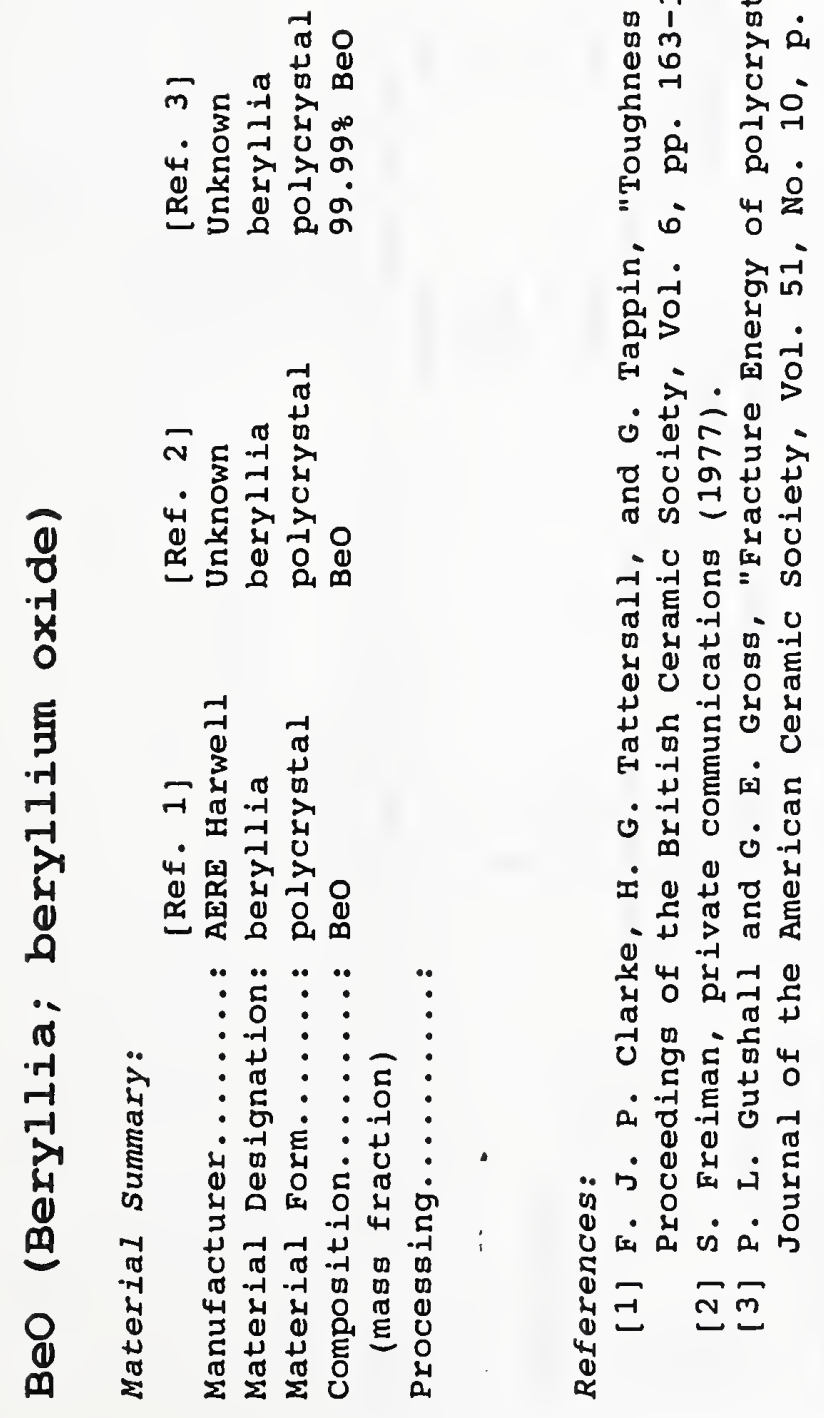

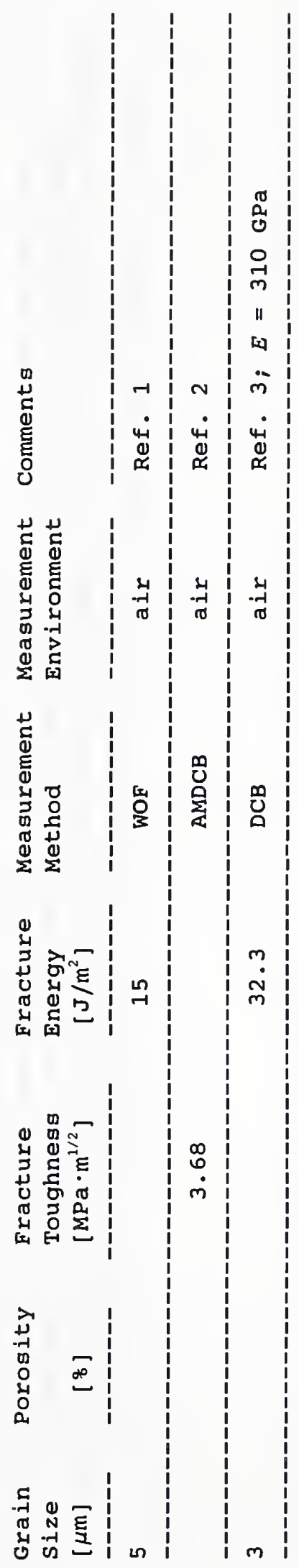

$\dot{n}$ 

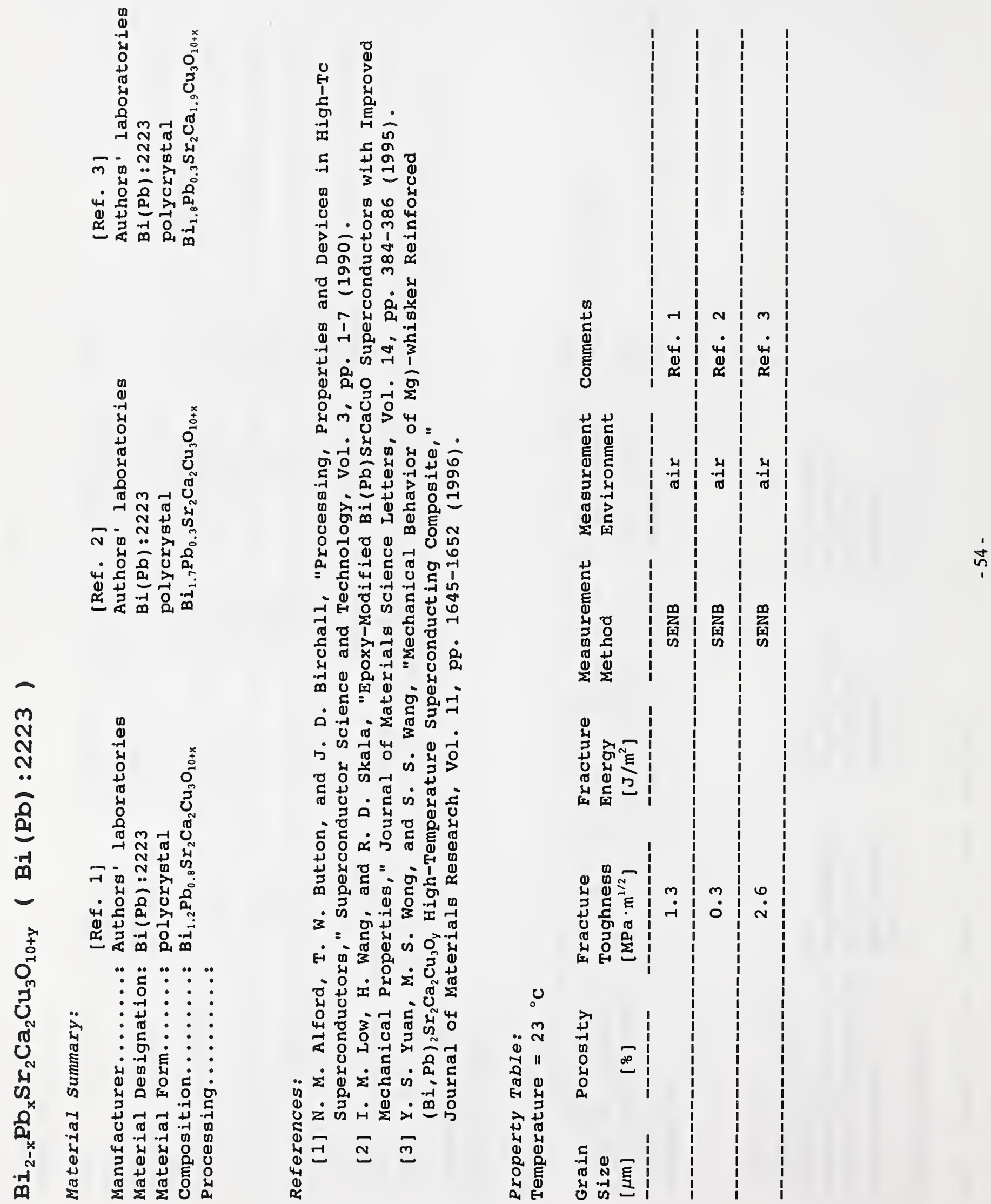

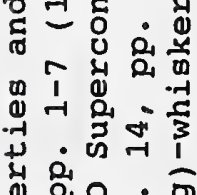

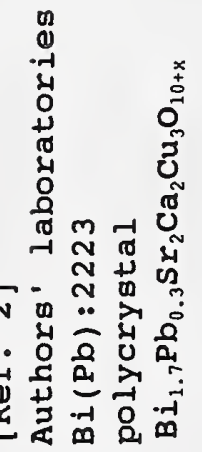

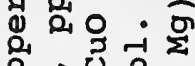

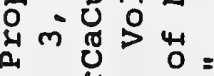

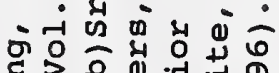

牙造势

क

0ั

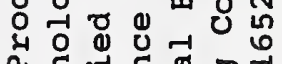

돈

证

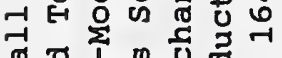

ฮี व

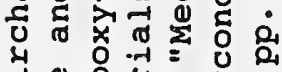

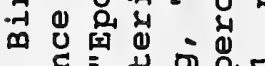

.

म

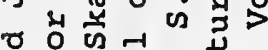

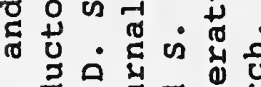

- حٓ

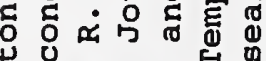

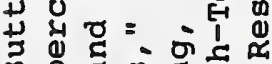

ค.

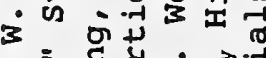

$\therefore$.

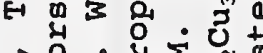

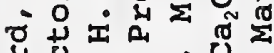

0

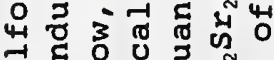

द ठु

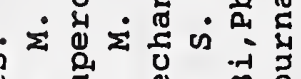

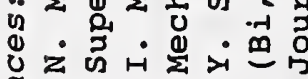

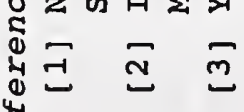
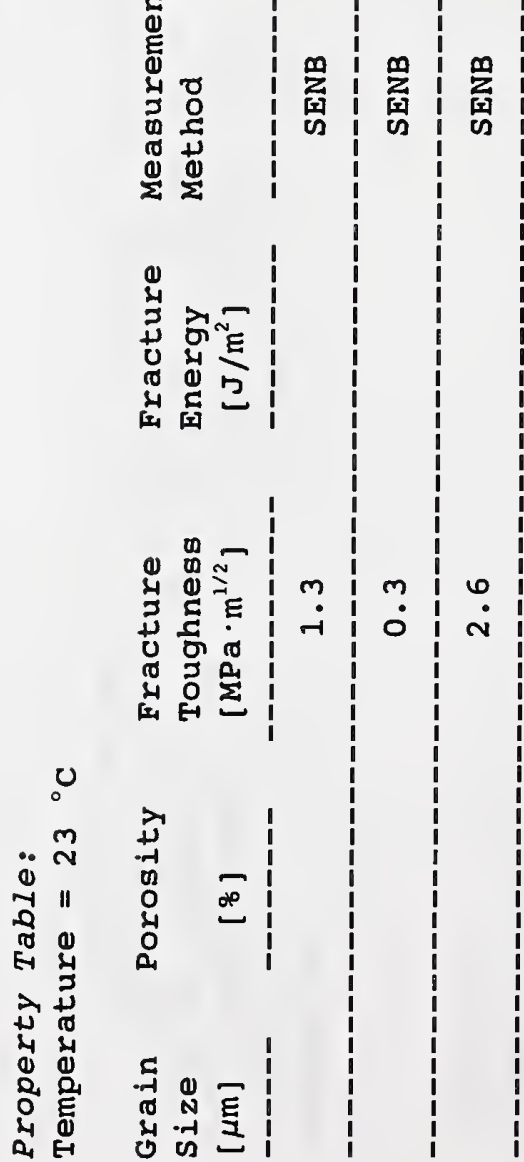

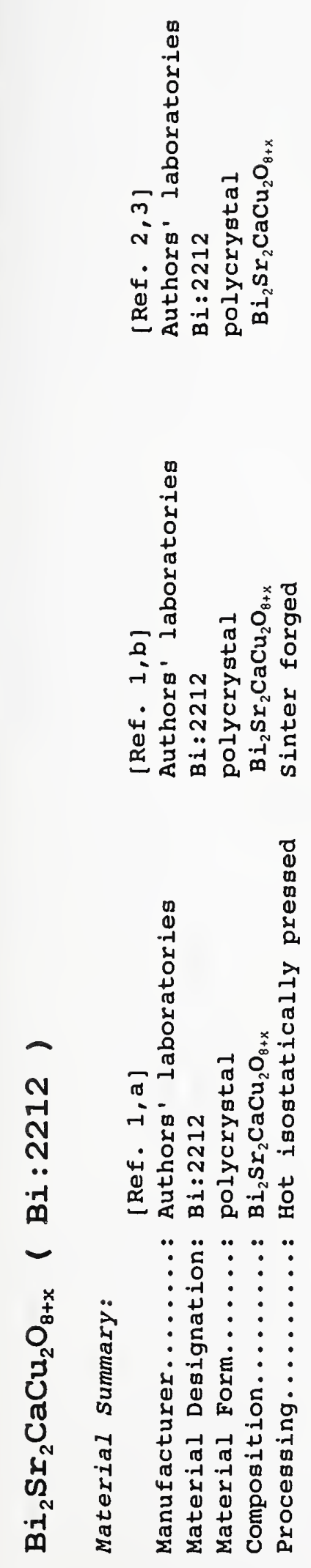

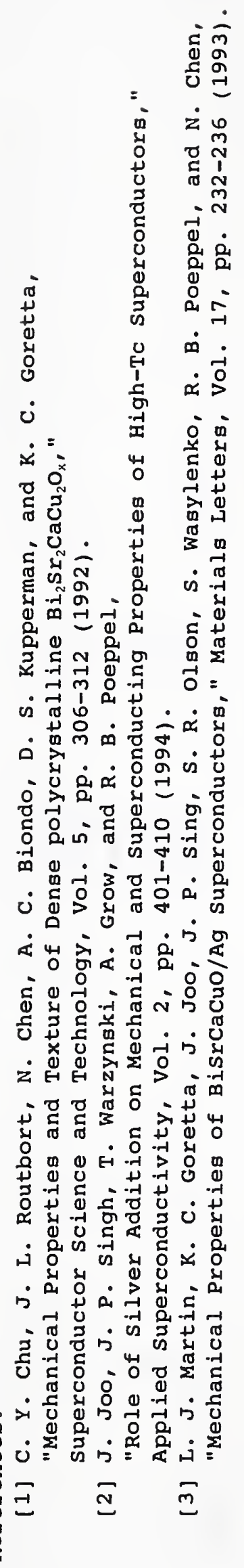




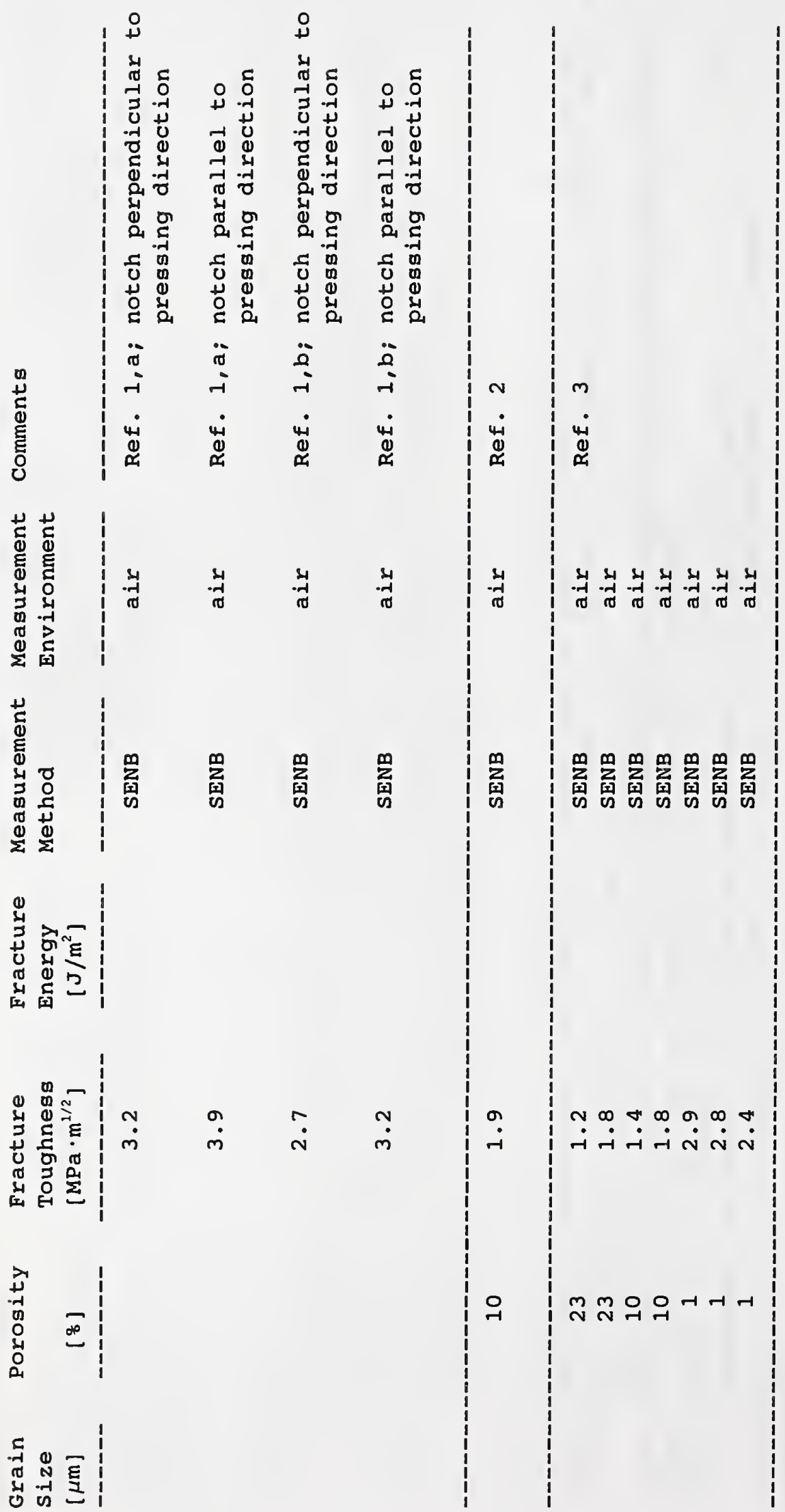




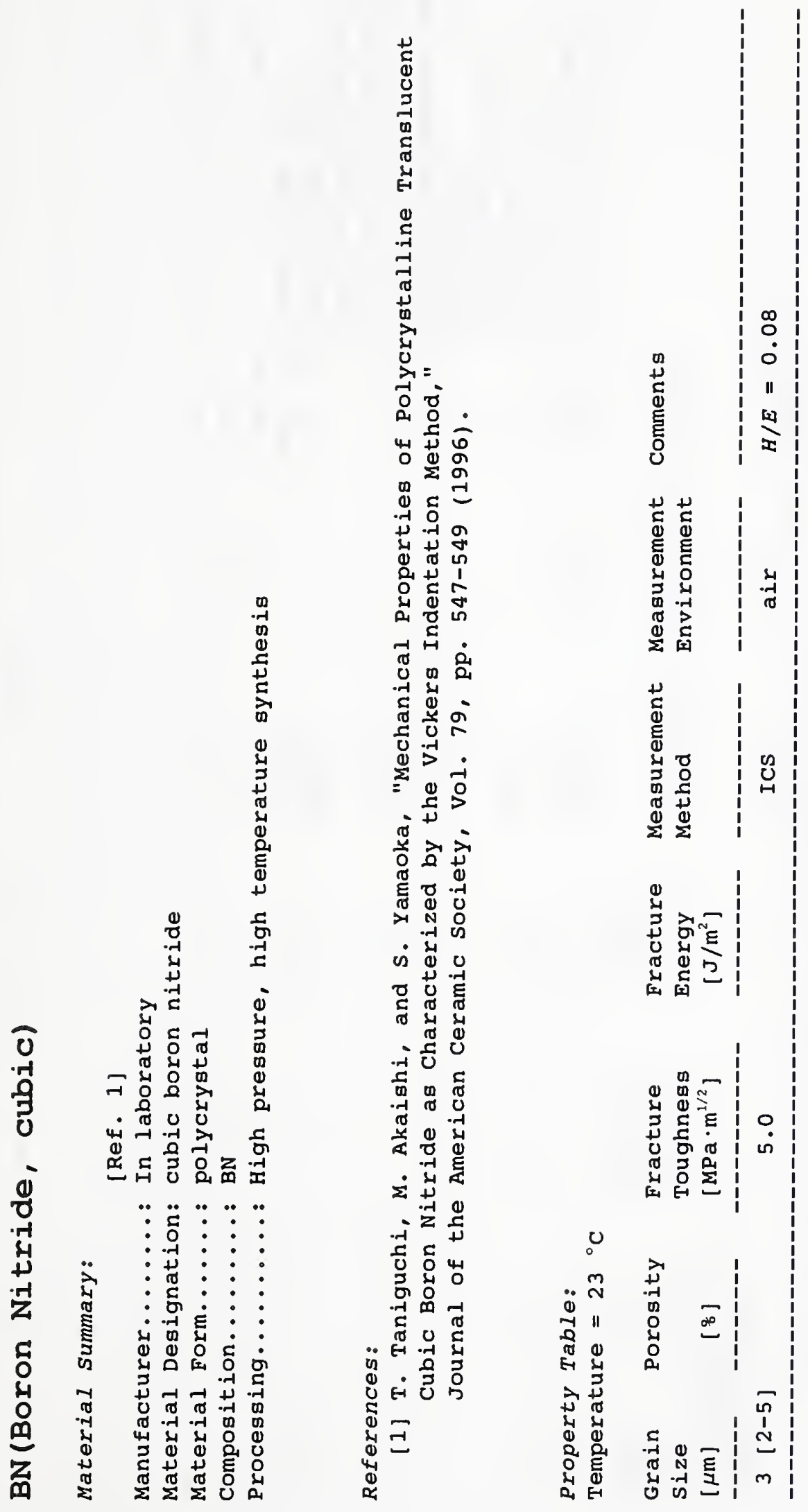




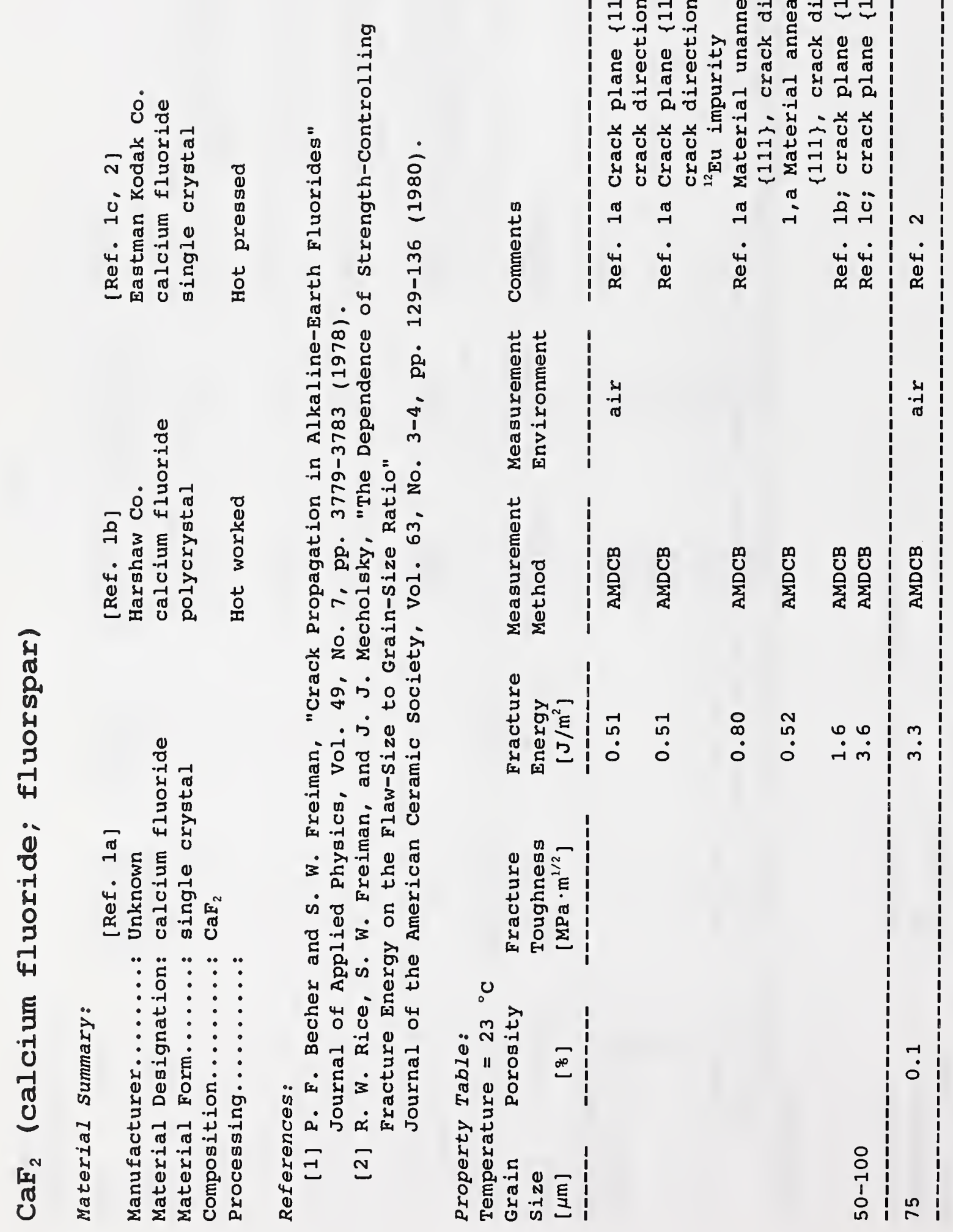




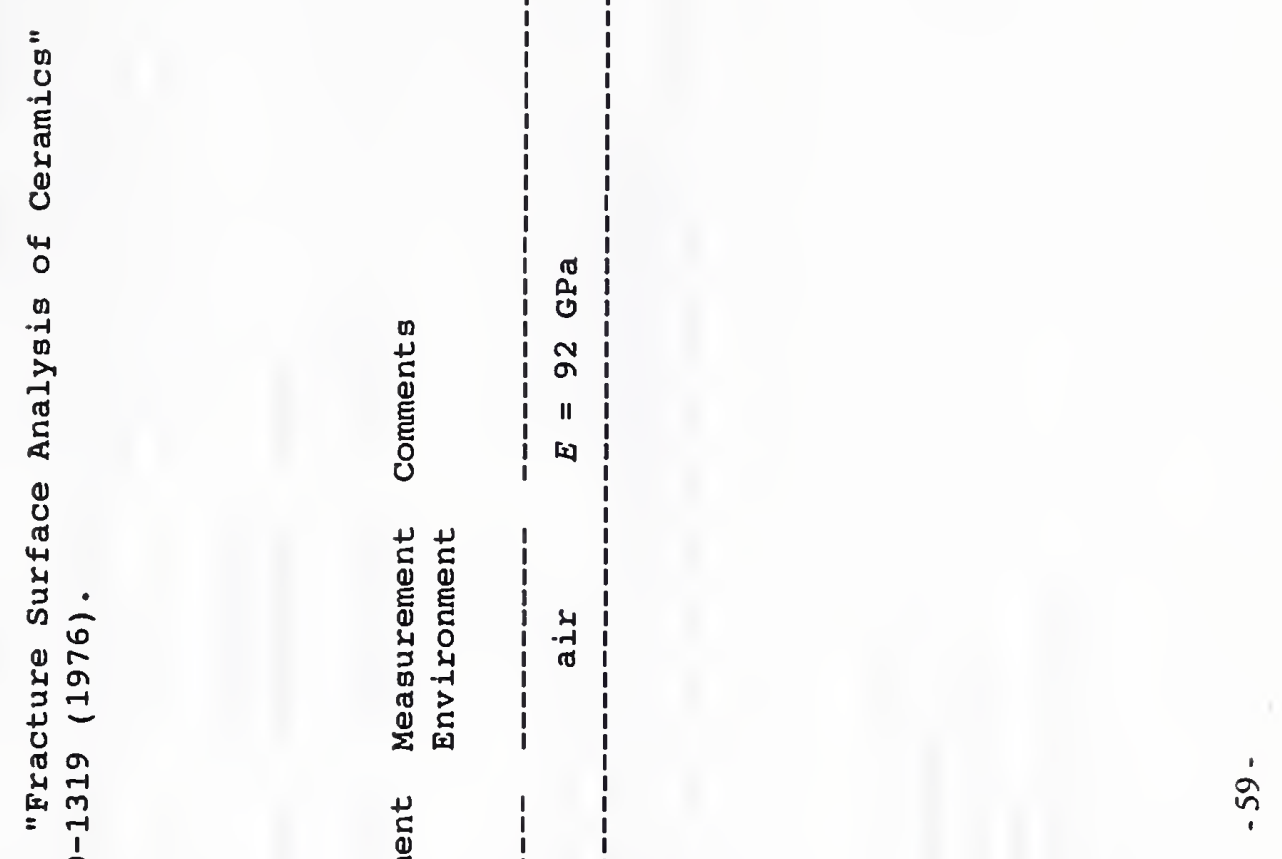

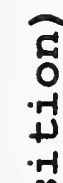

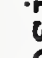

(2)

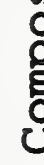

列

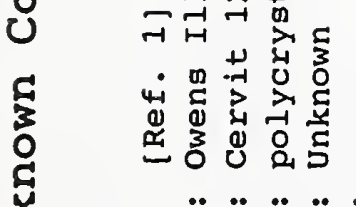

要

ह

N

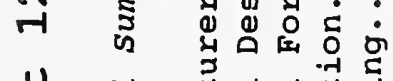

薄

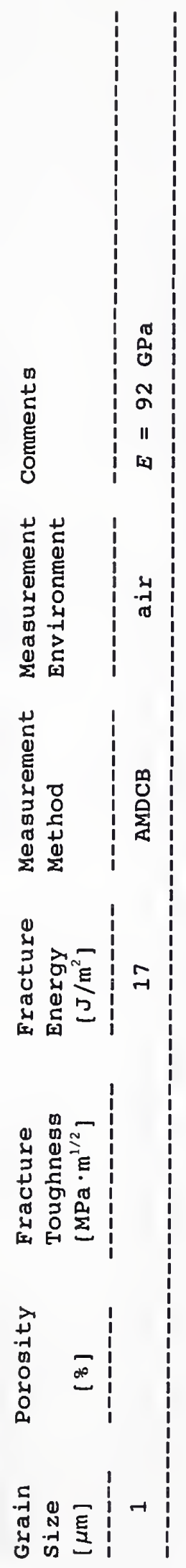




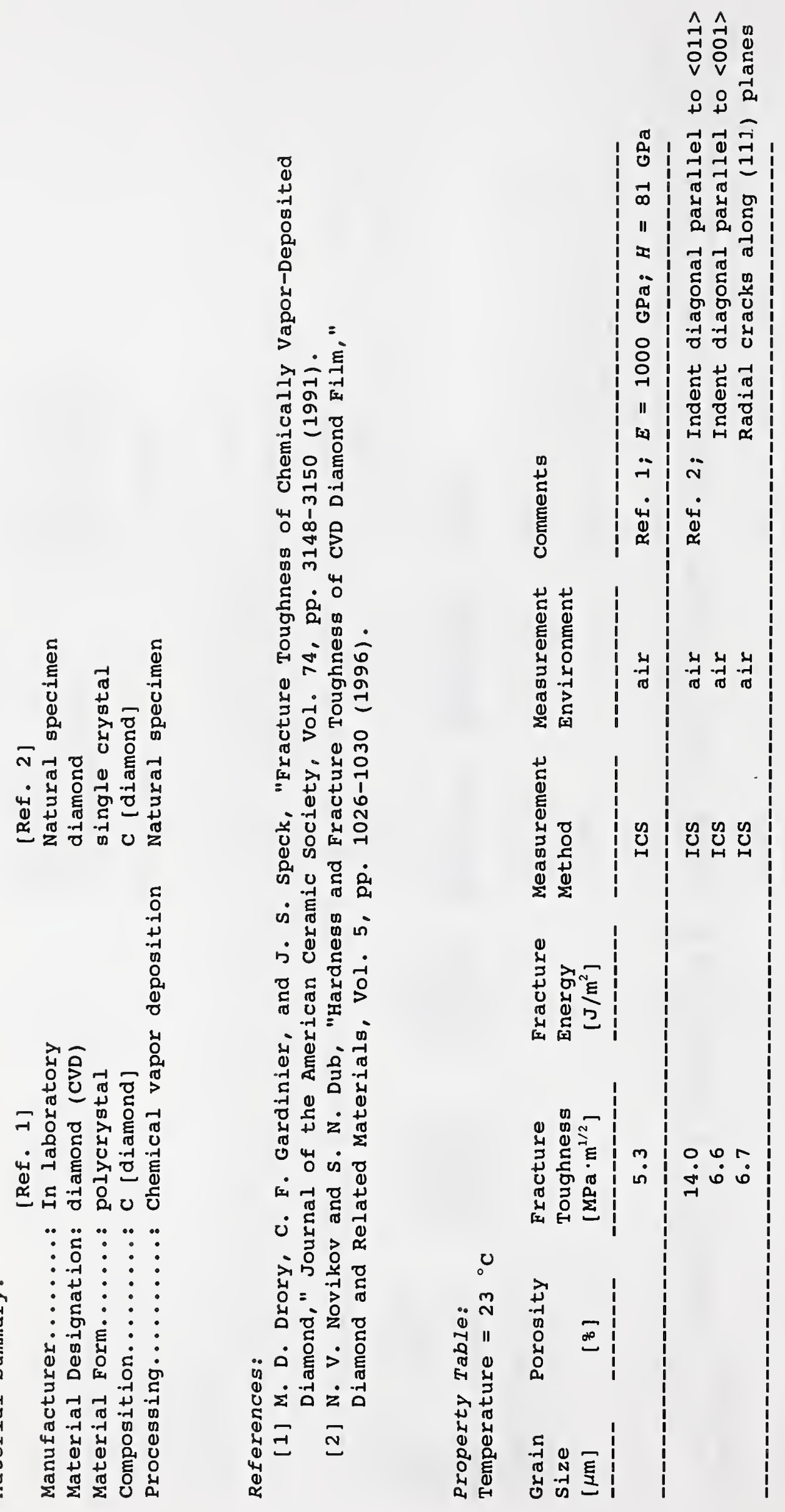




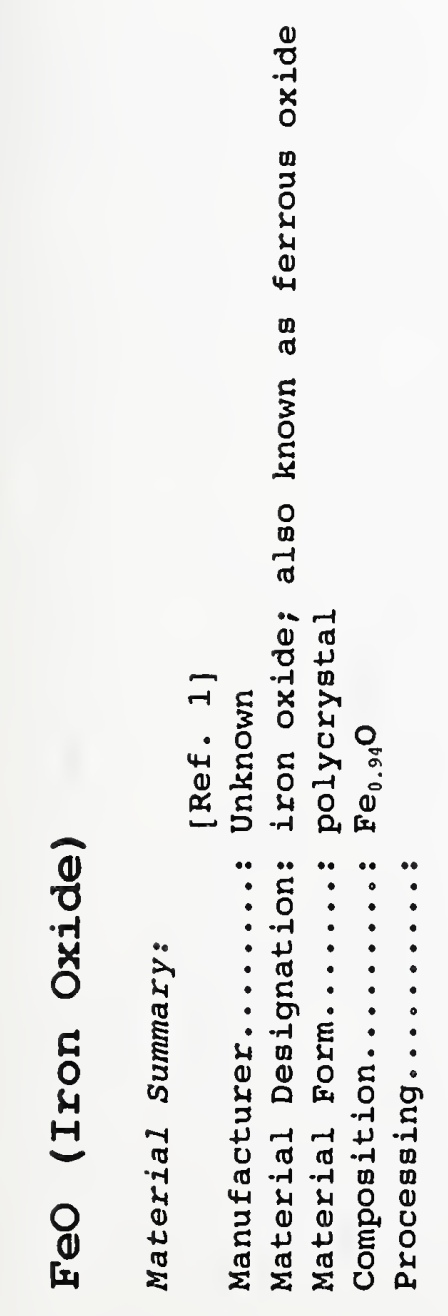

.
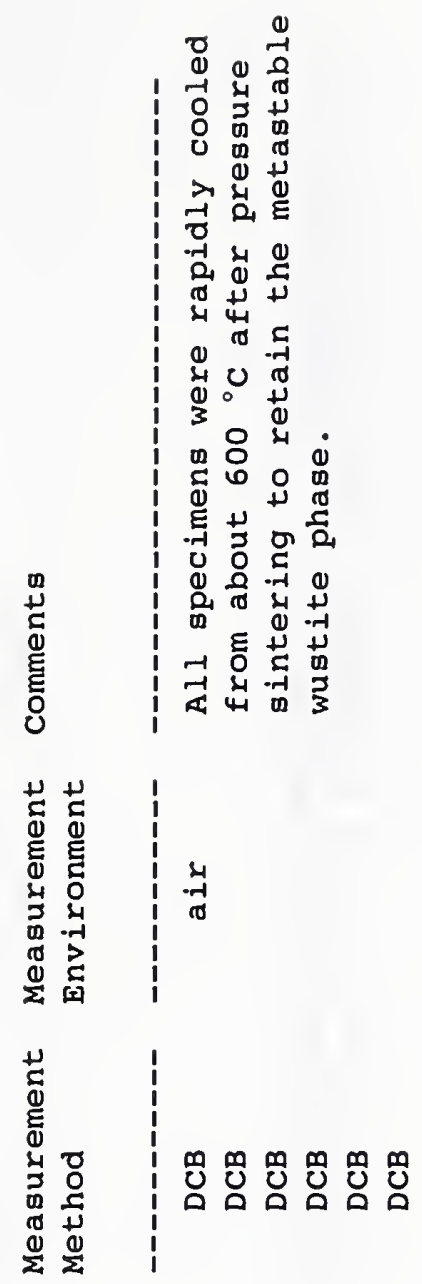

넉

สิ 1

ปั กิ

雚这

㟧

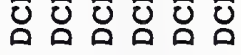

远

赑告

.

¿ ठै

质出
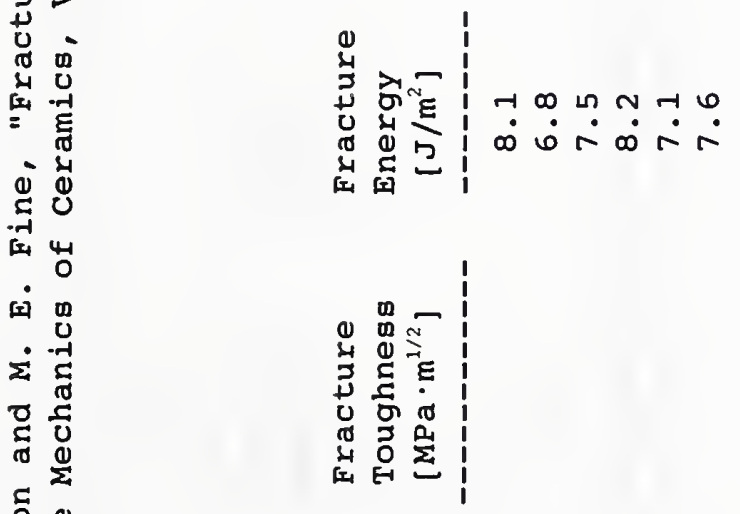

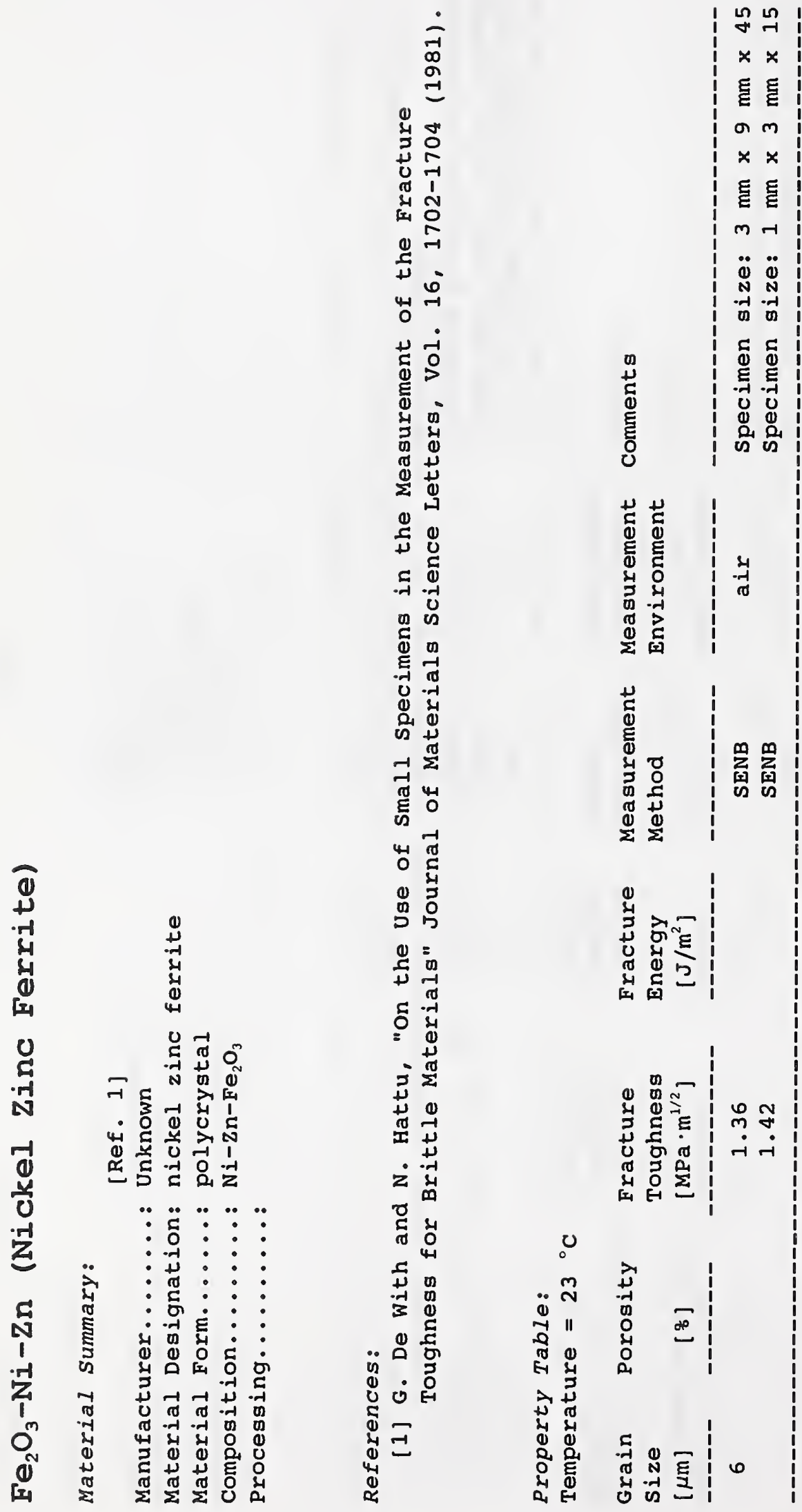

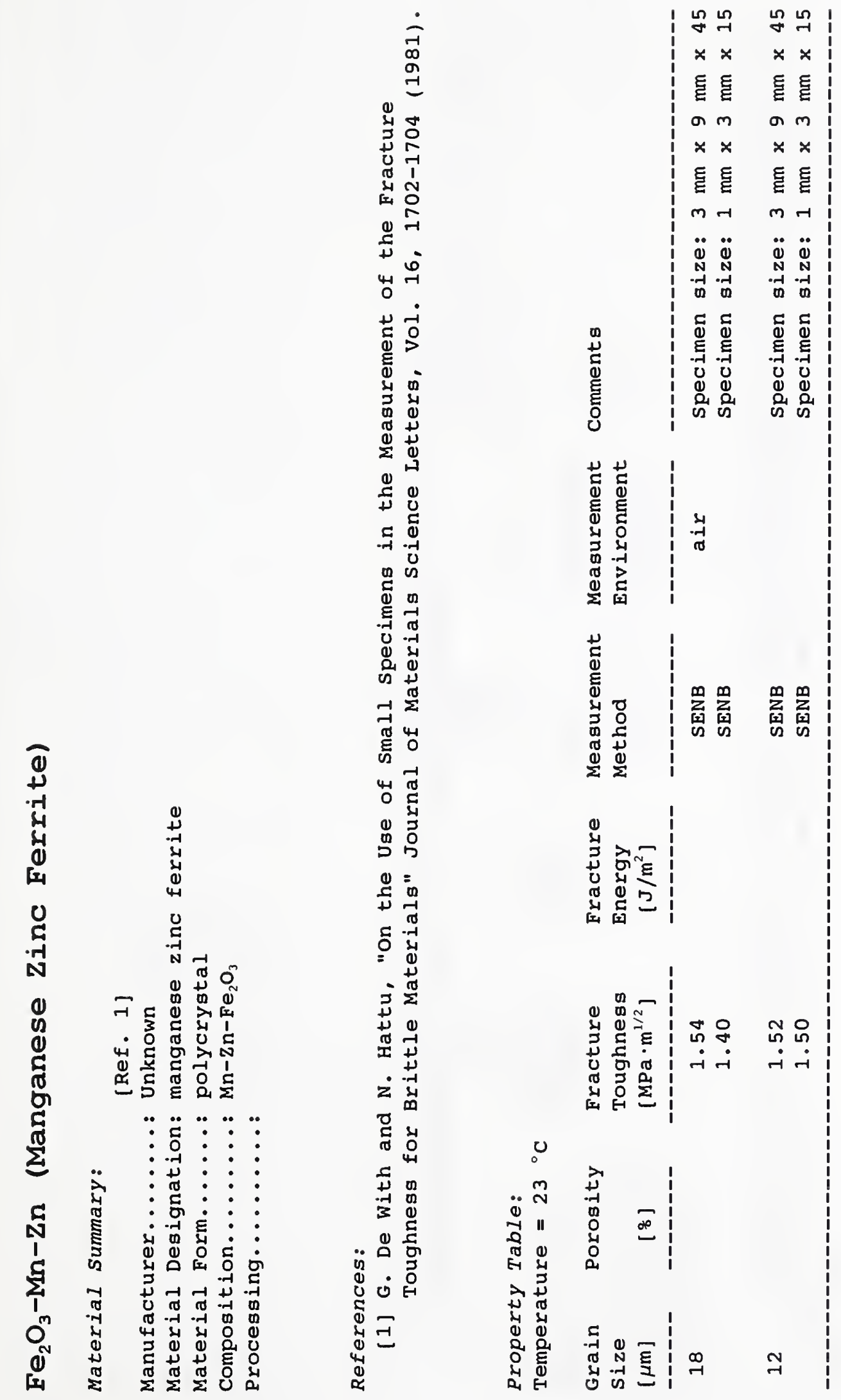


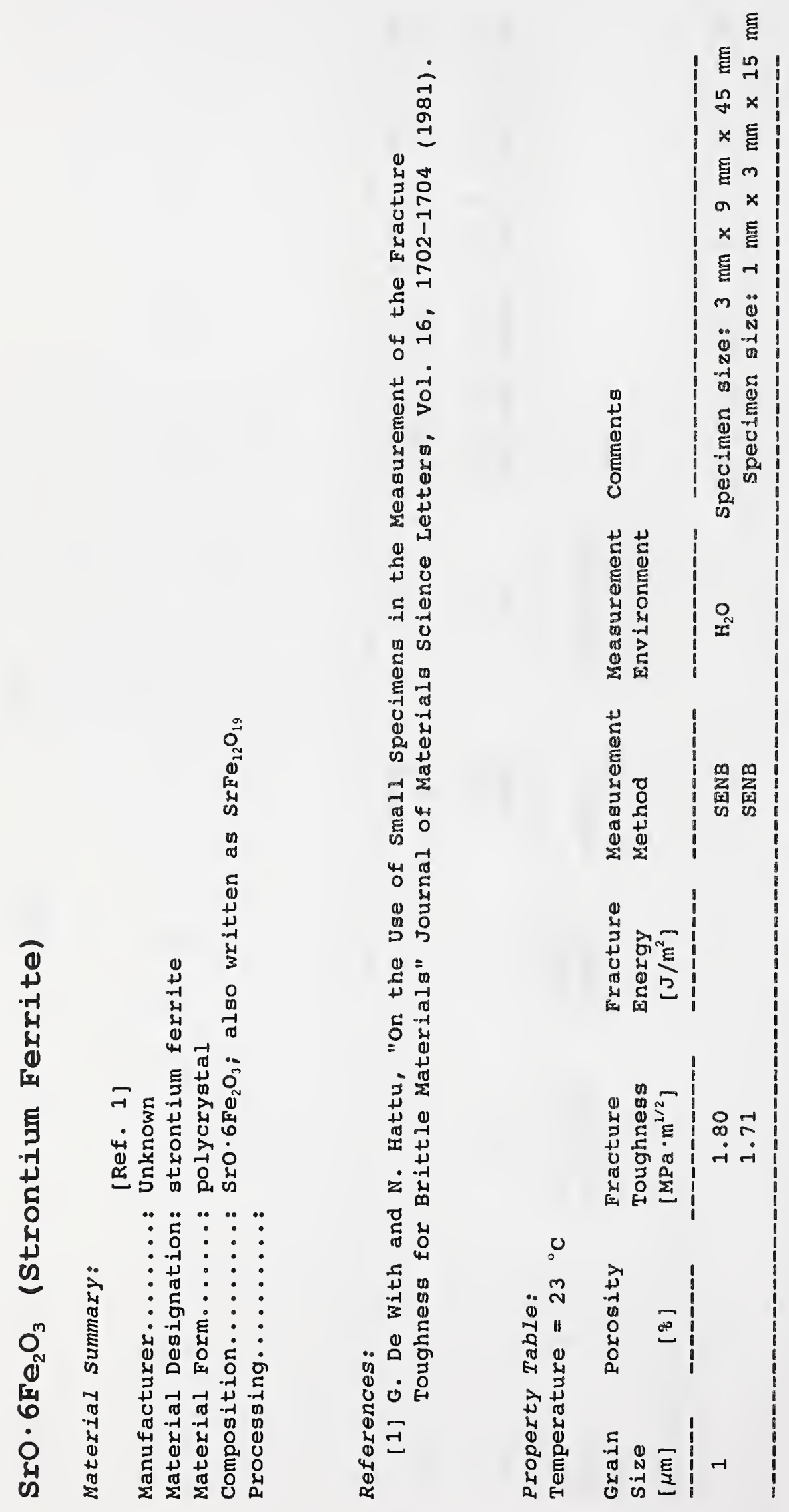




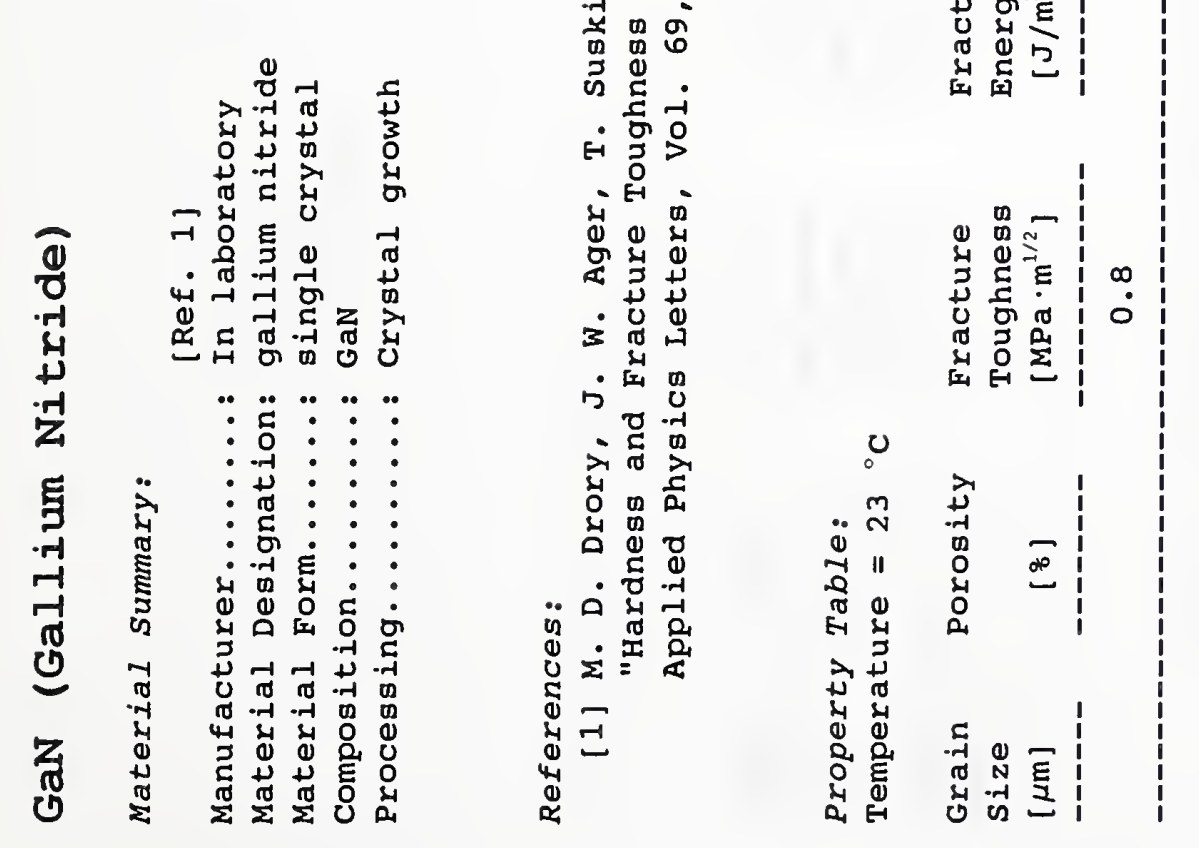




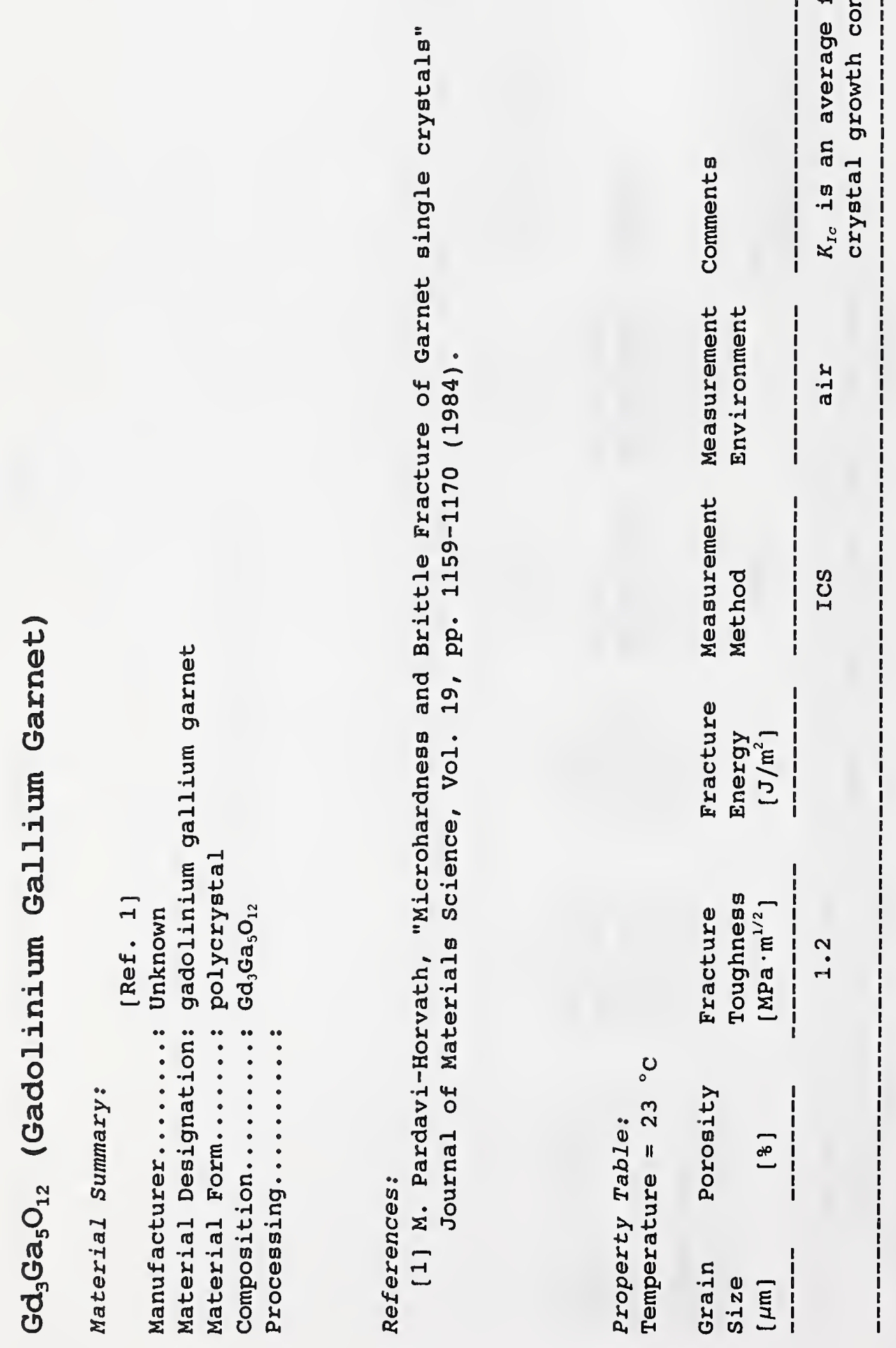




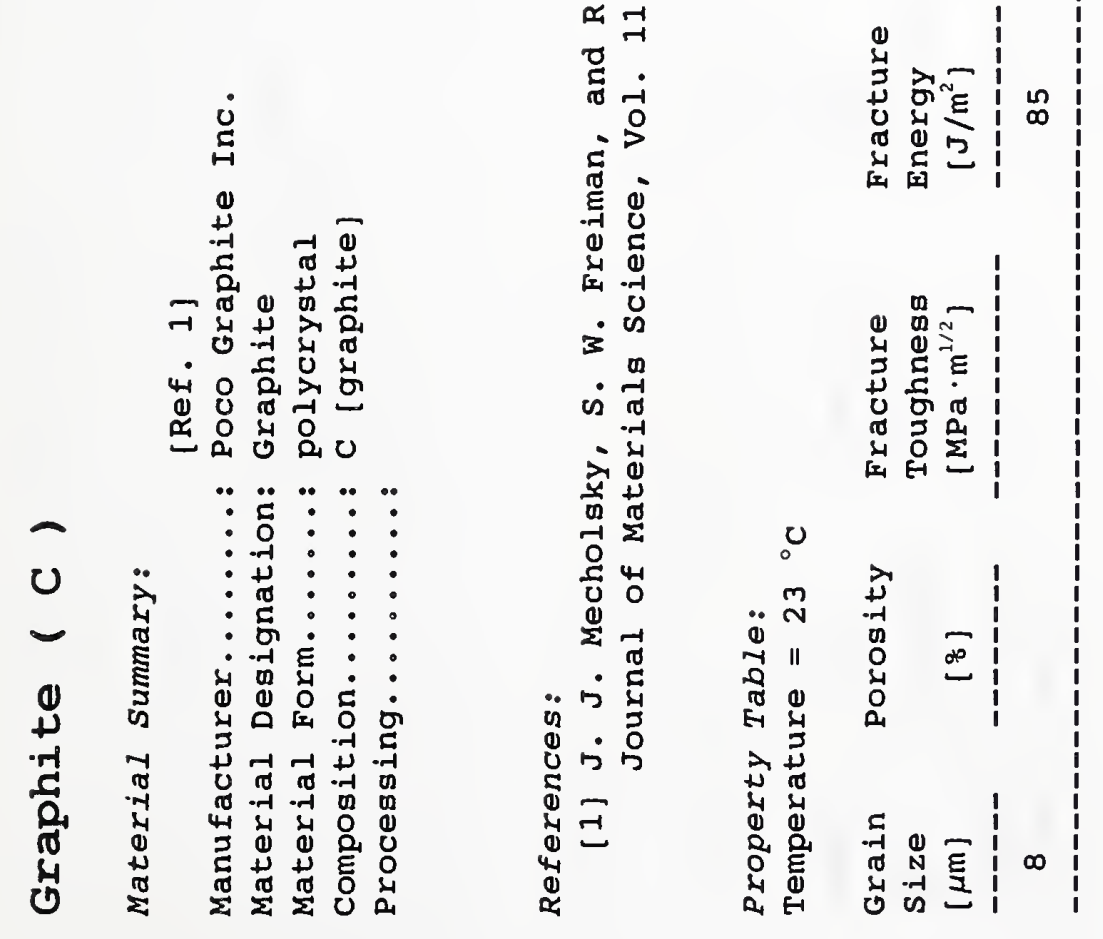




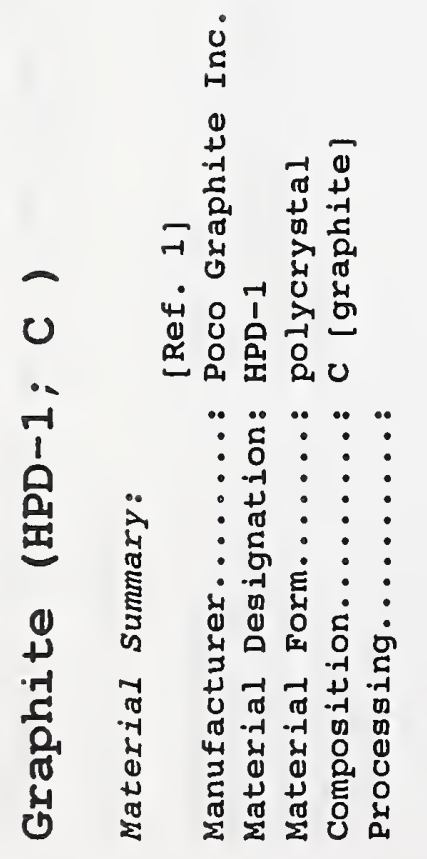

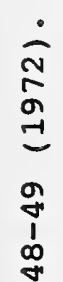
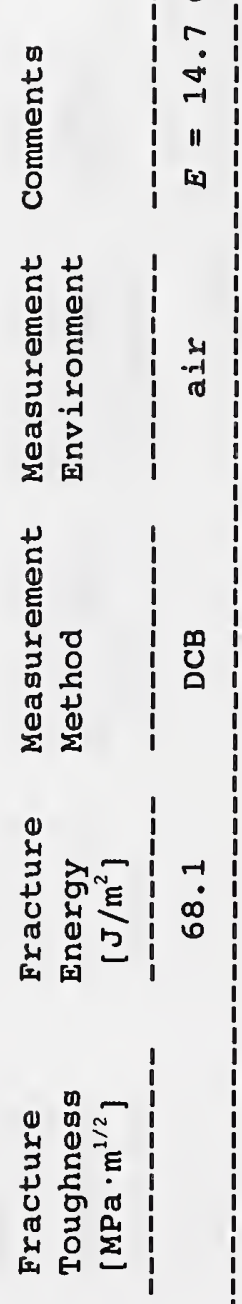

这站
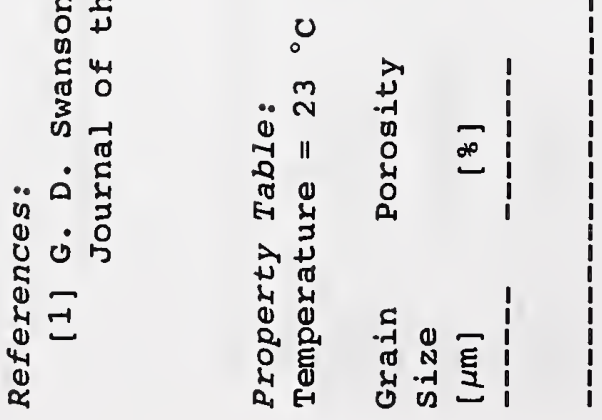


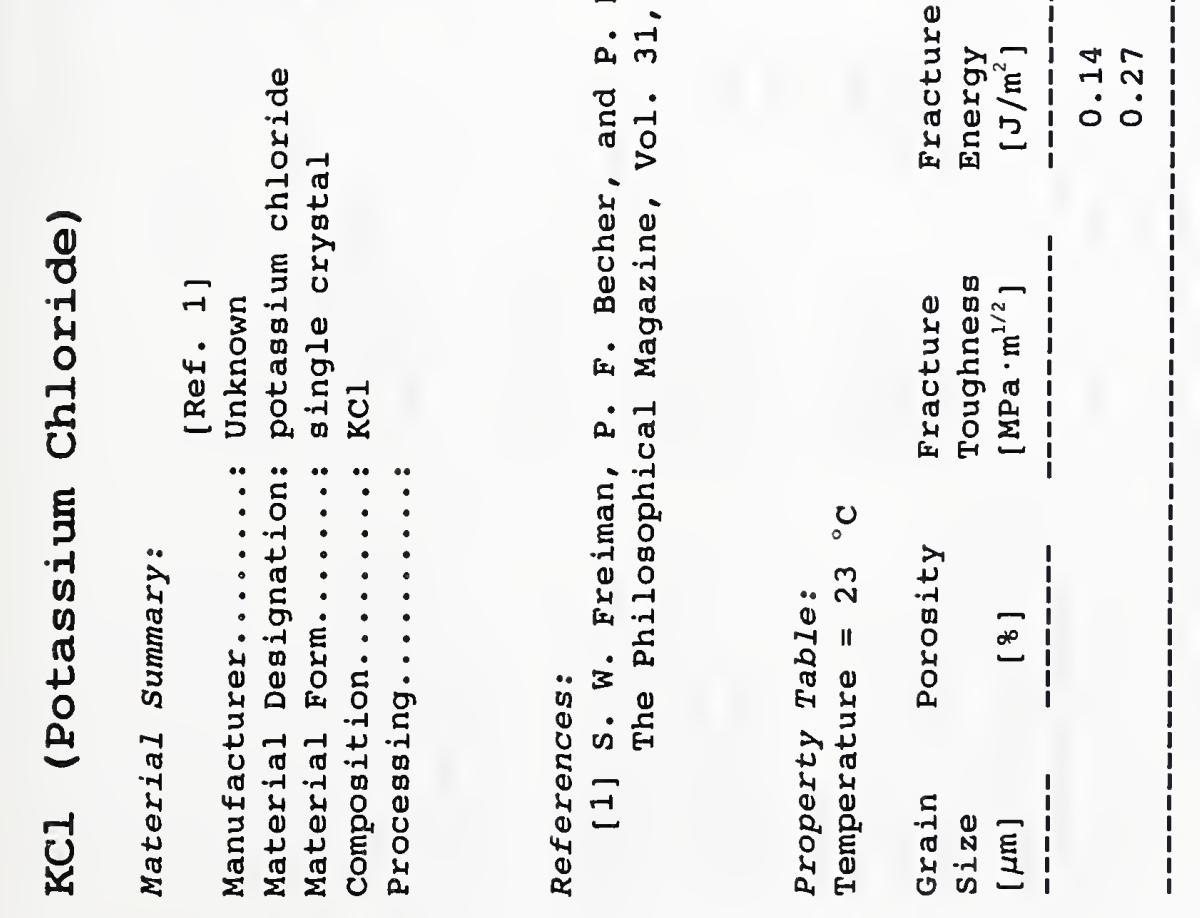



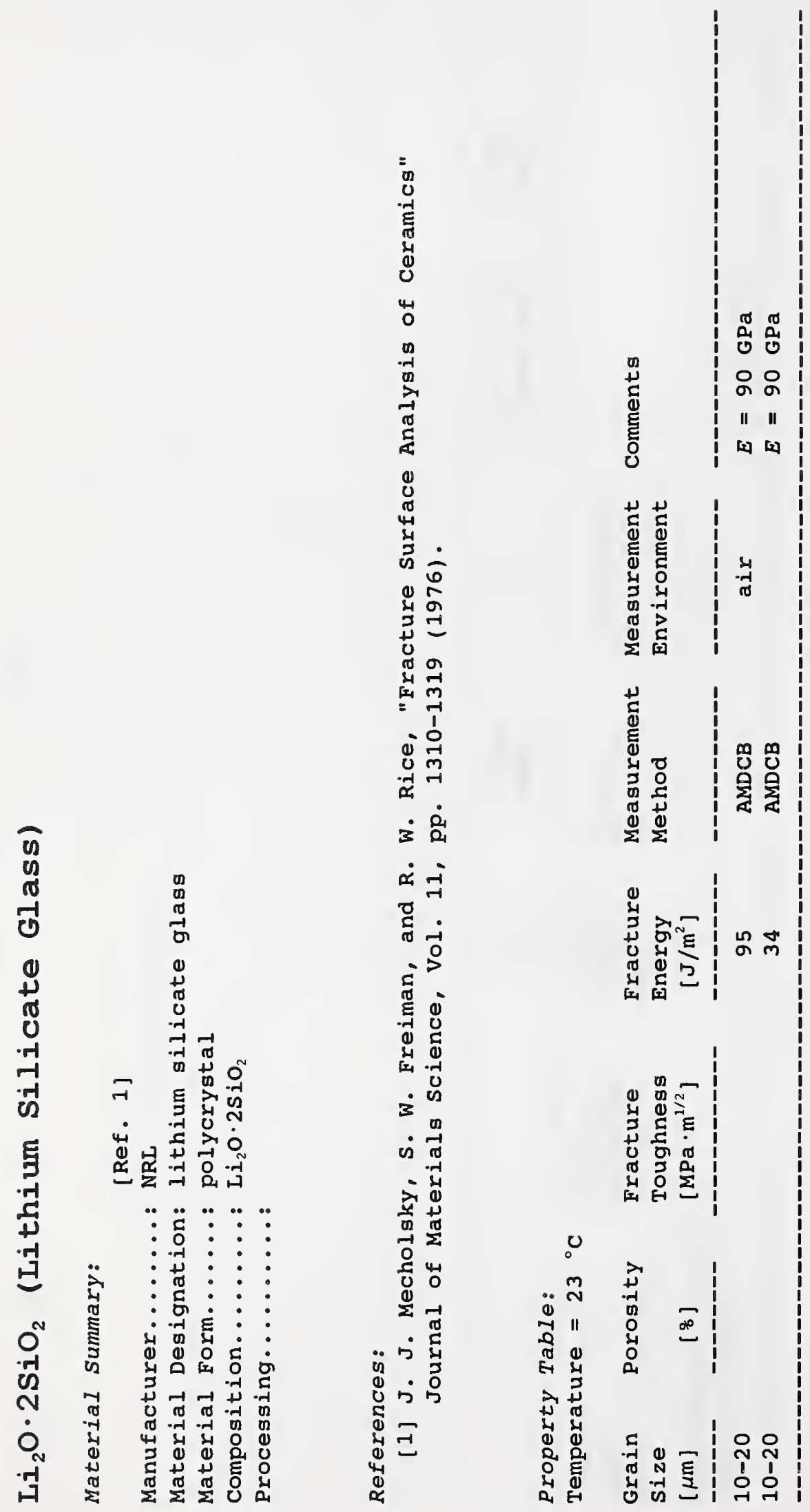


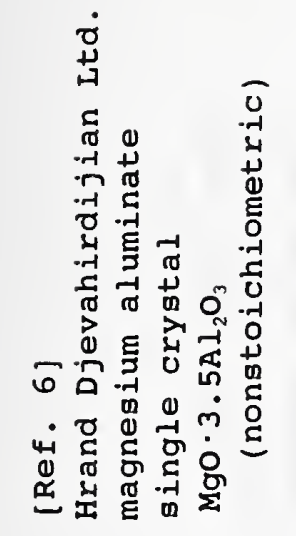

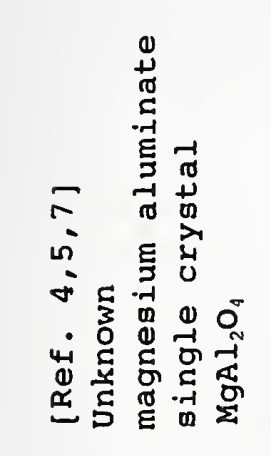

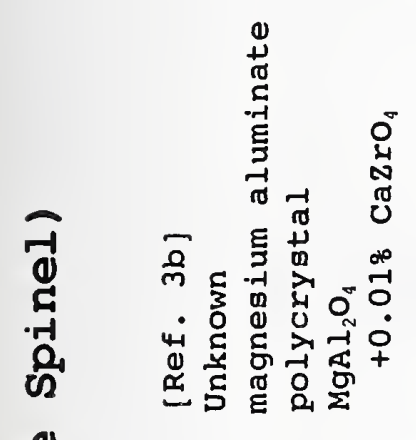

西

(1)

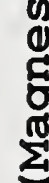
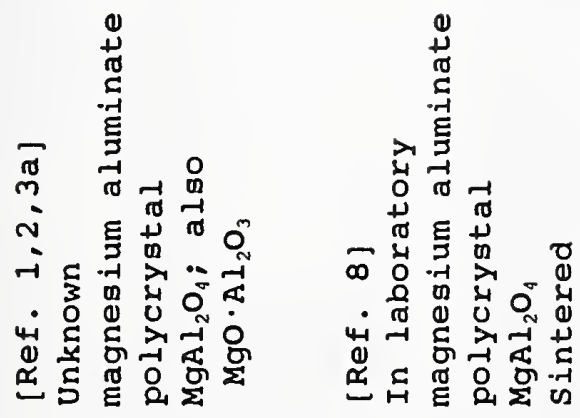

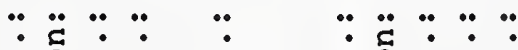

:

: 元: :

- ह: :

:न है:

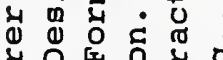

峞苟 质

प)

का न-

山

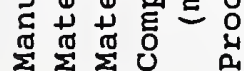

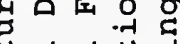
सन न न d 岁落

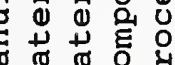

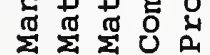

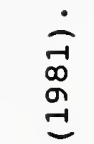

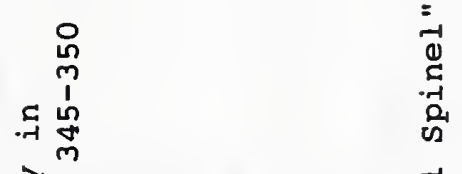

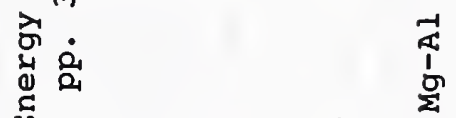

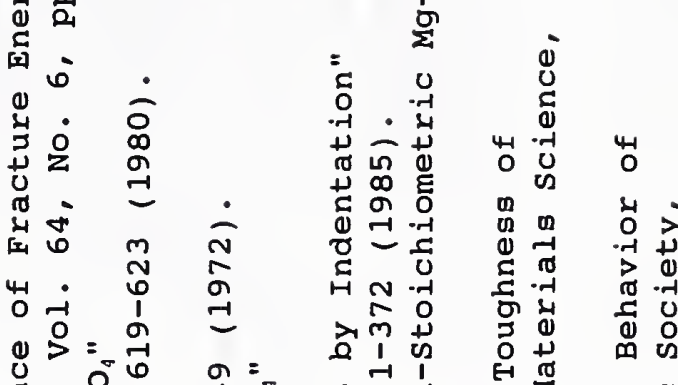

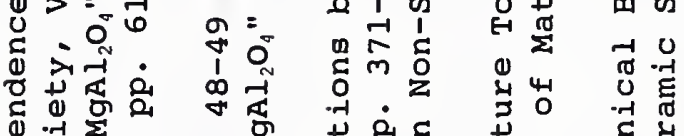

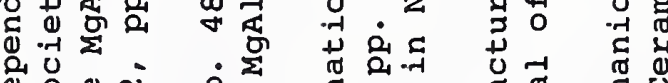

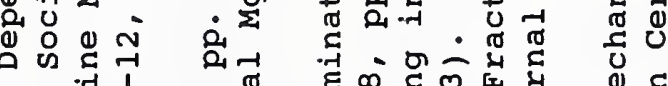
0.

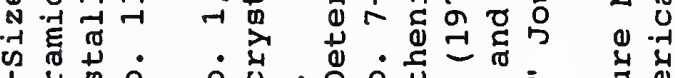

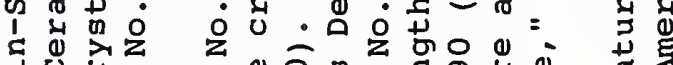

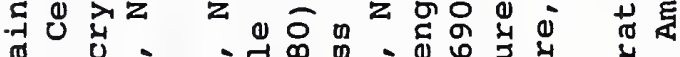

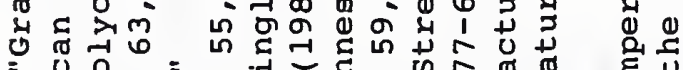

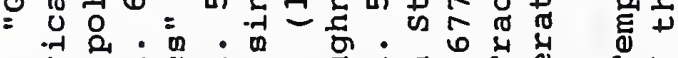

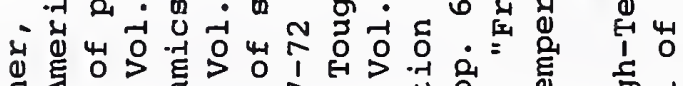

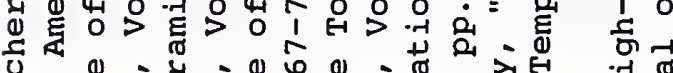

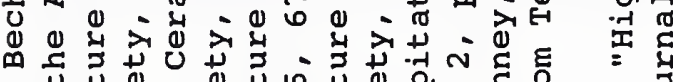

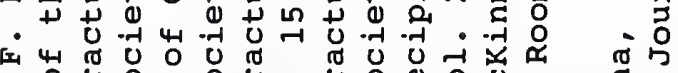

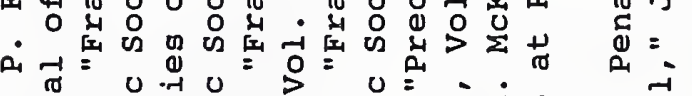
ช

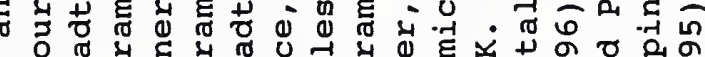

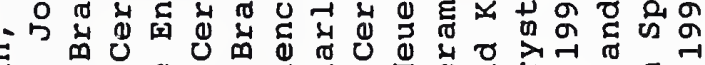
ส

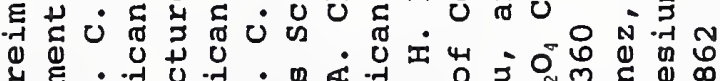

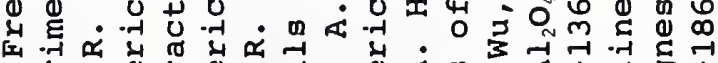
员

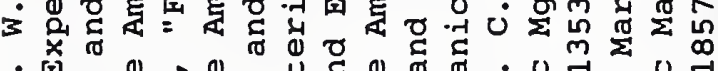

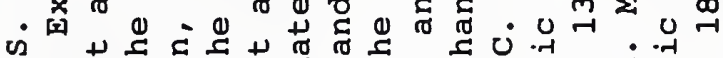

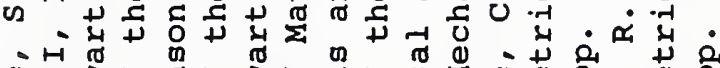

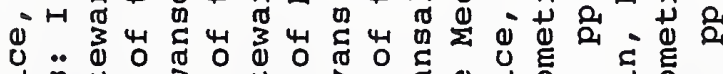

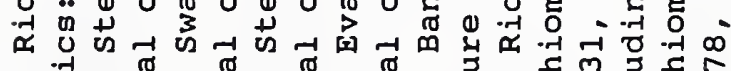

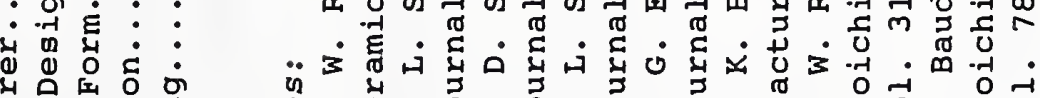

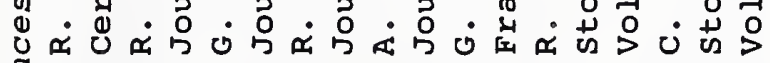

岁 


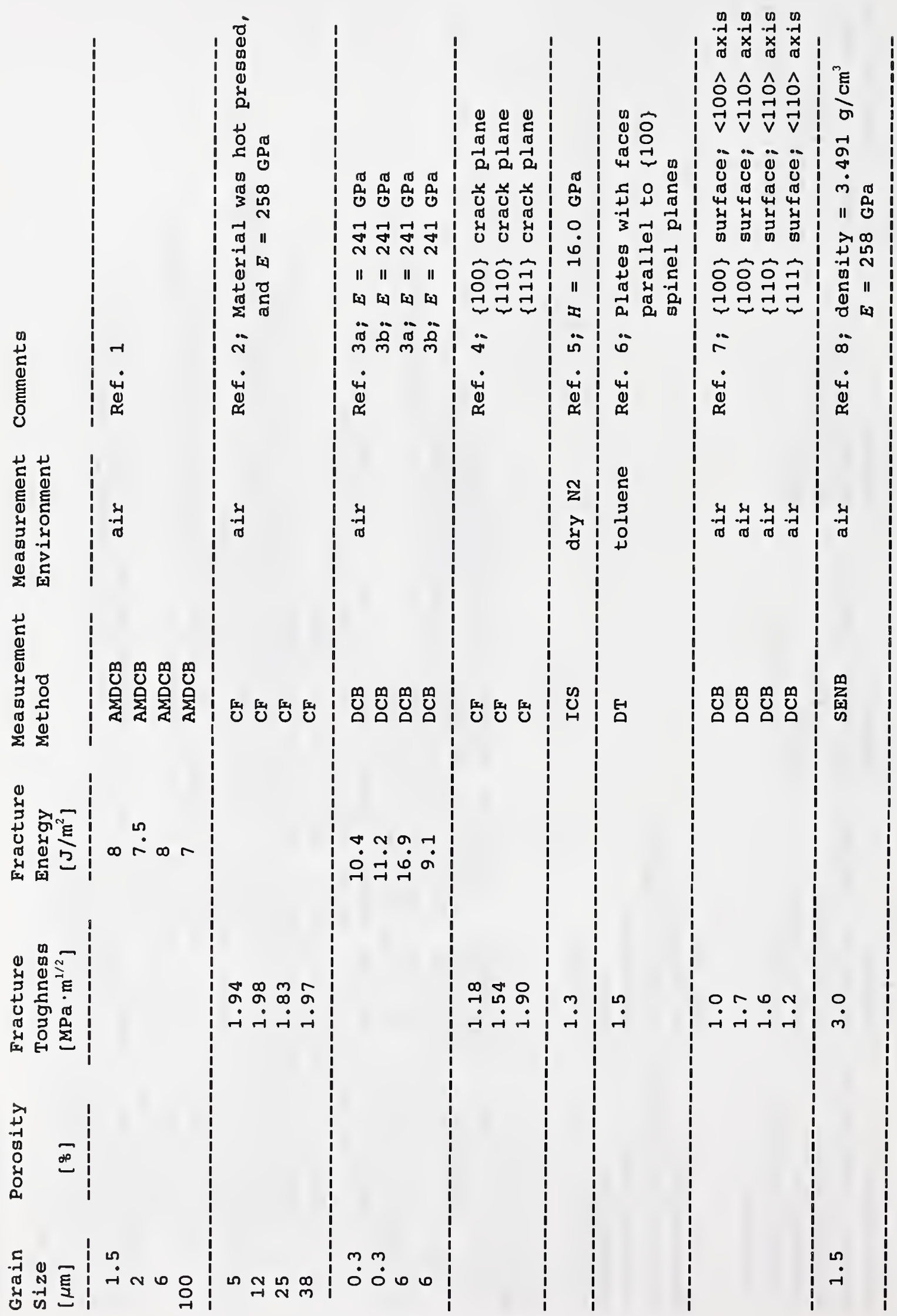



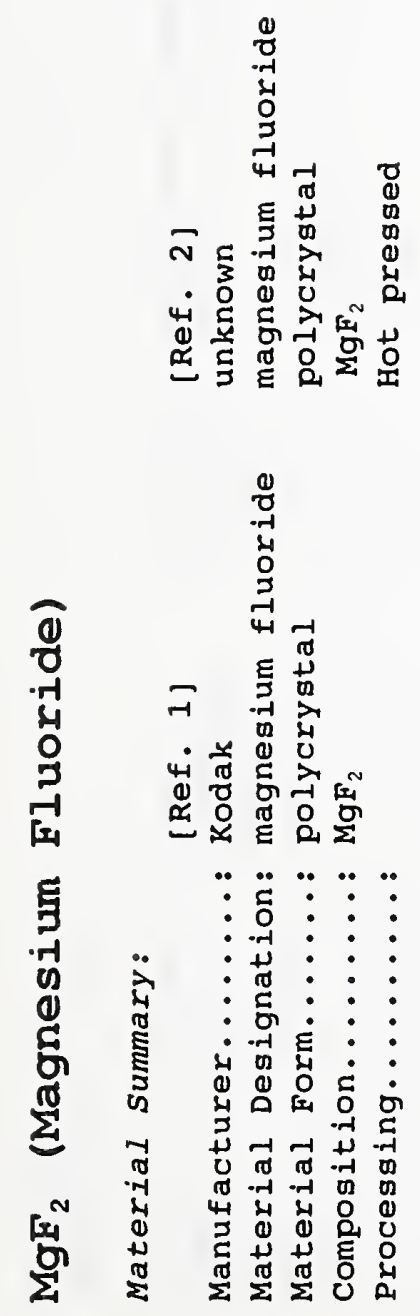

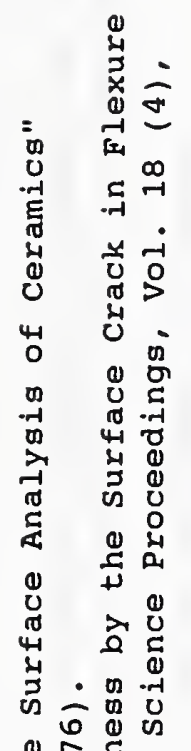

的。

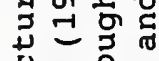

क 0

急

150

बi

車

. = 红

उ مि.

ن न न

开至

बi

它

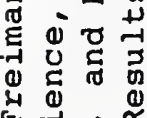

至

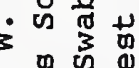

- 感出.

i $\cos _{-1}$ i

- 40. ¿

尊的柴

范范

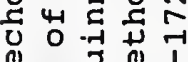

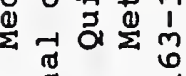

ij

艺

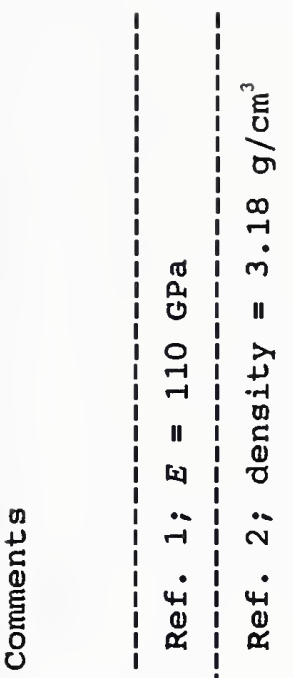

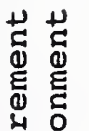

资

$\Rightarrow 4$

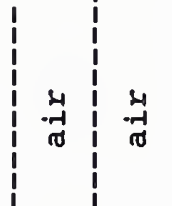

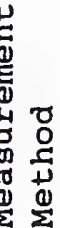

$\stackrel{2}{2}$ 

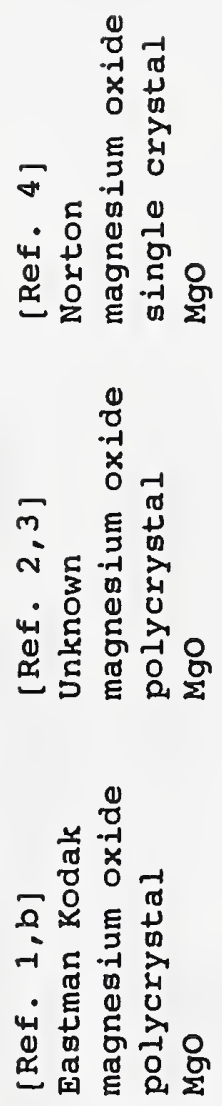

苛

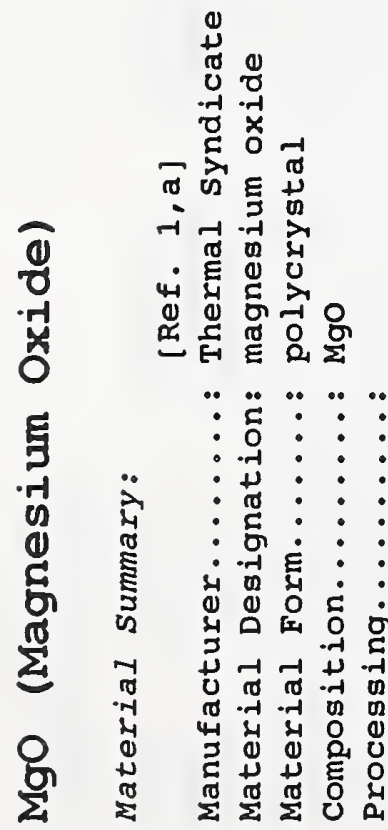

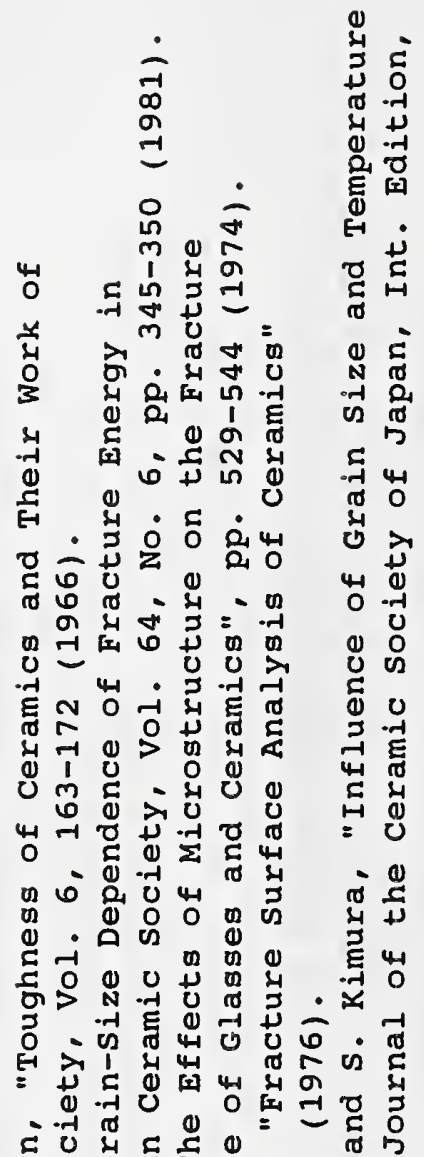

न 0 ठ त

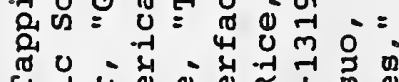
स्- हิ

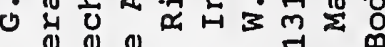
ฮ

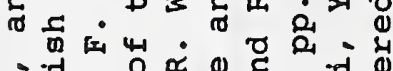

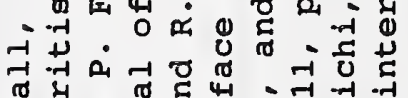
के

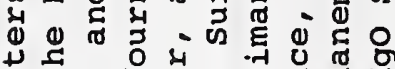
出

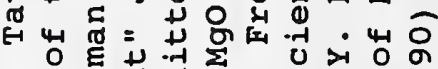
णे है व थ है क

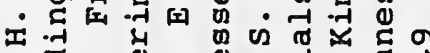

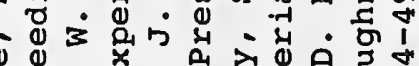
॥ ป

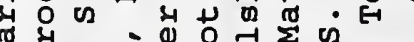

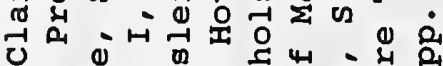

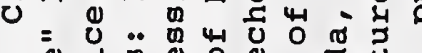

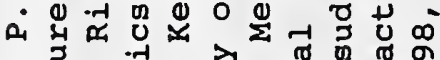

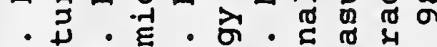

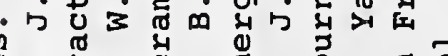

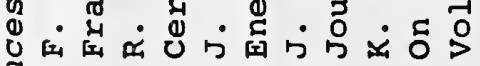

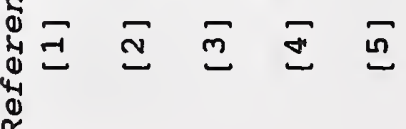




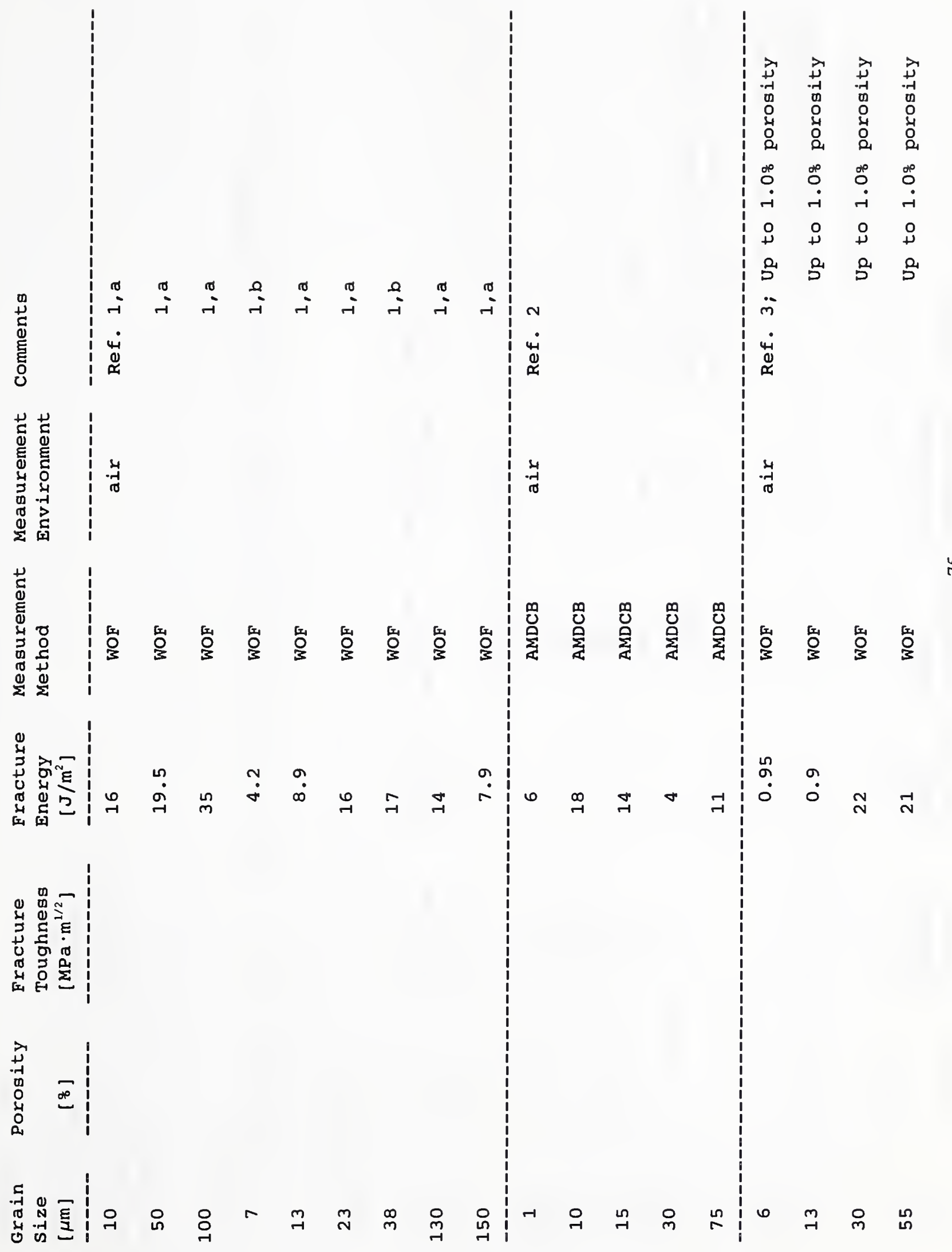




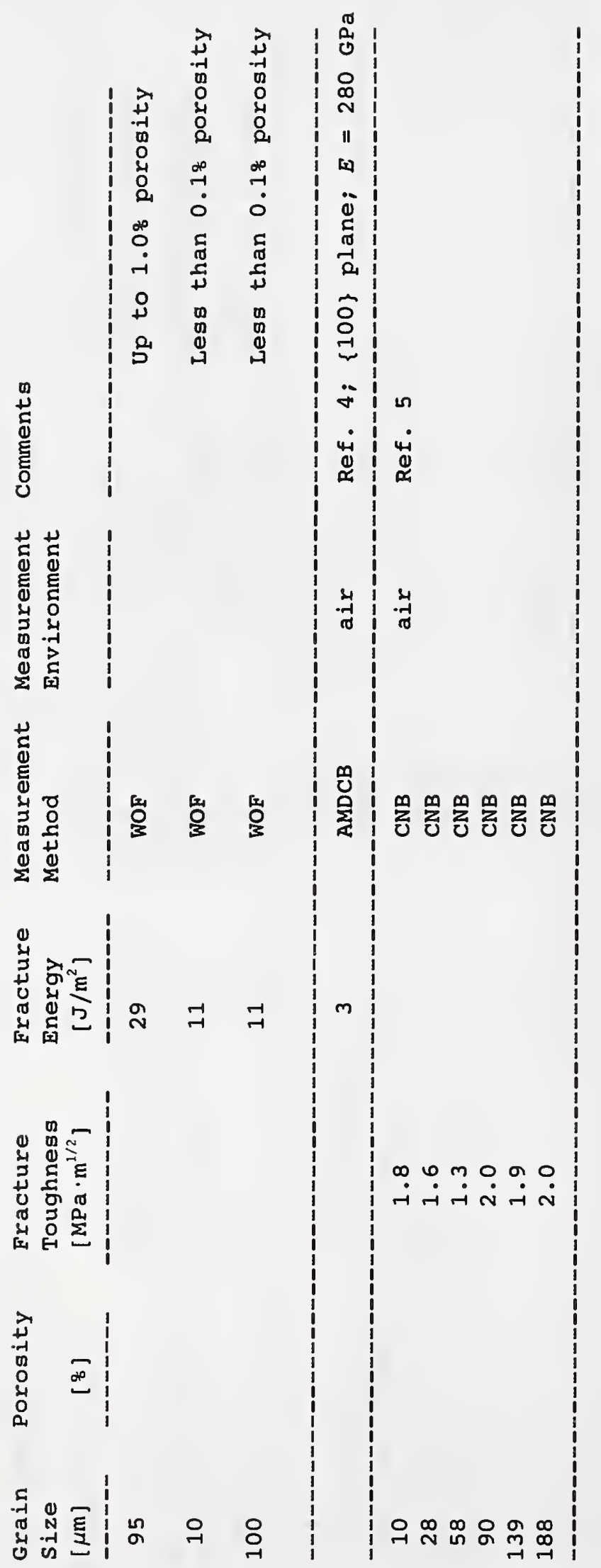

$\frac{1}{1}$ 


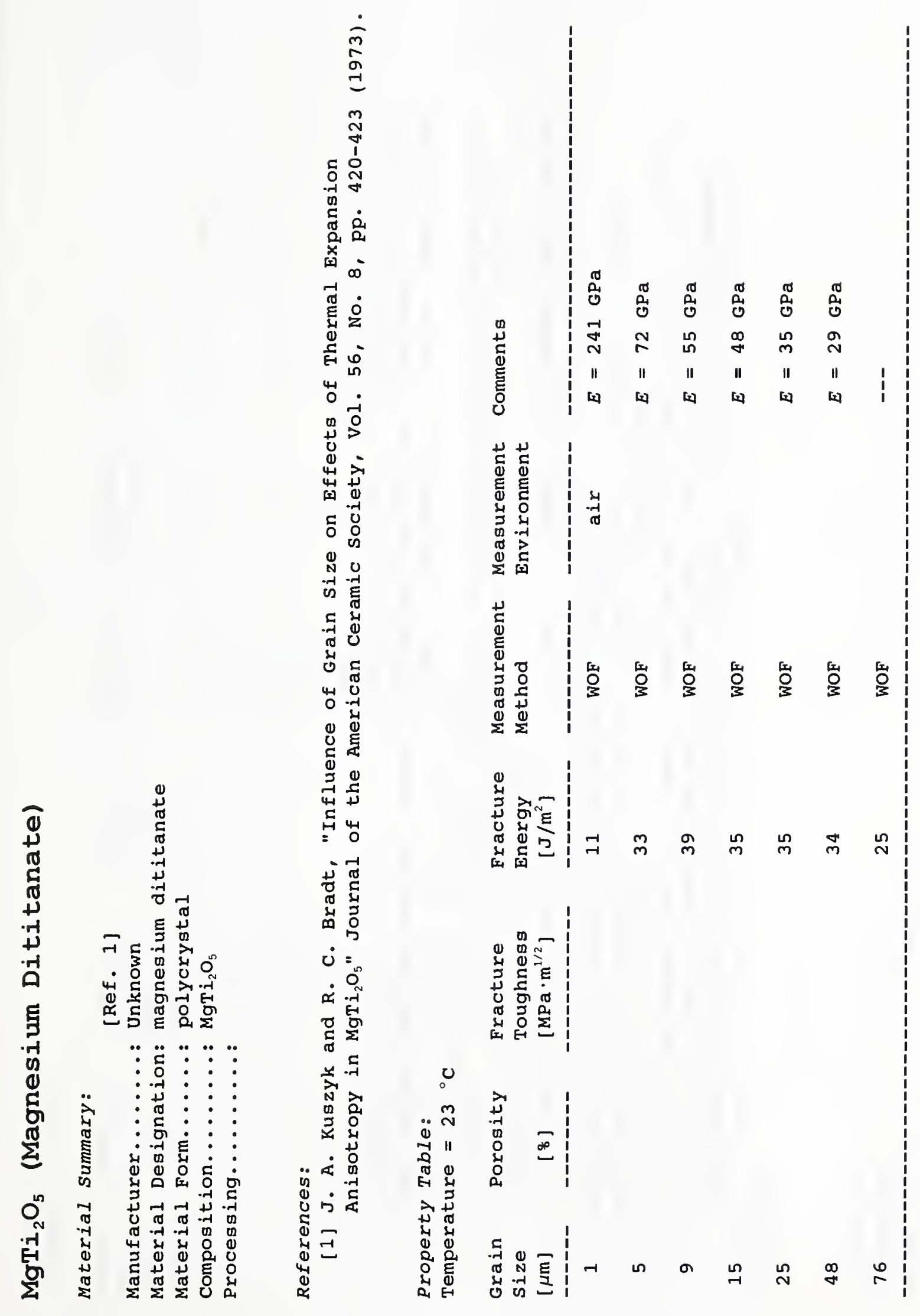




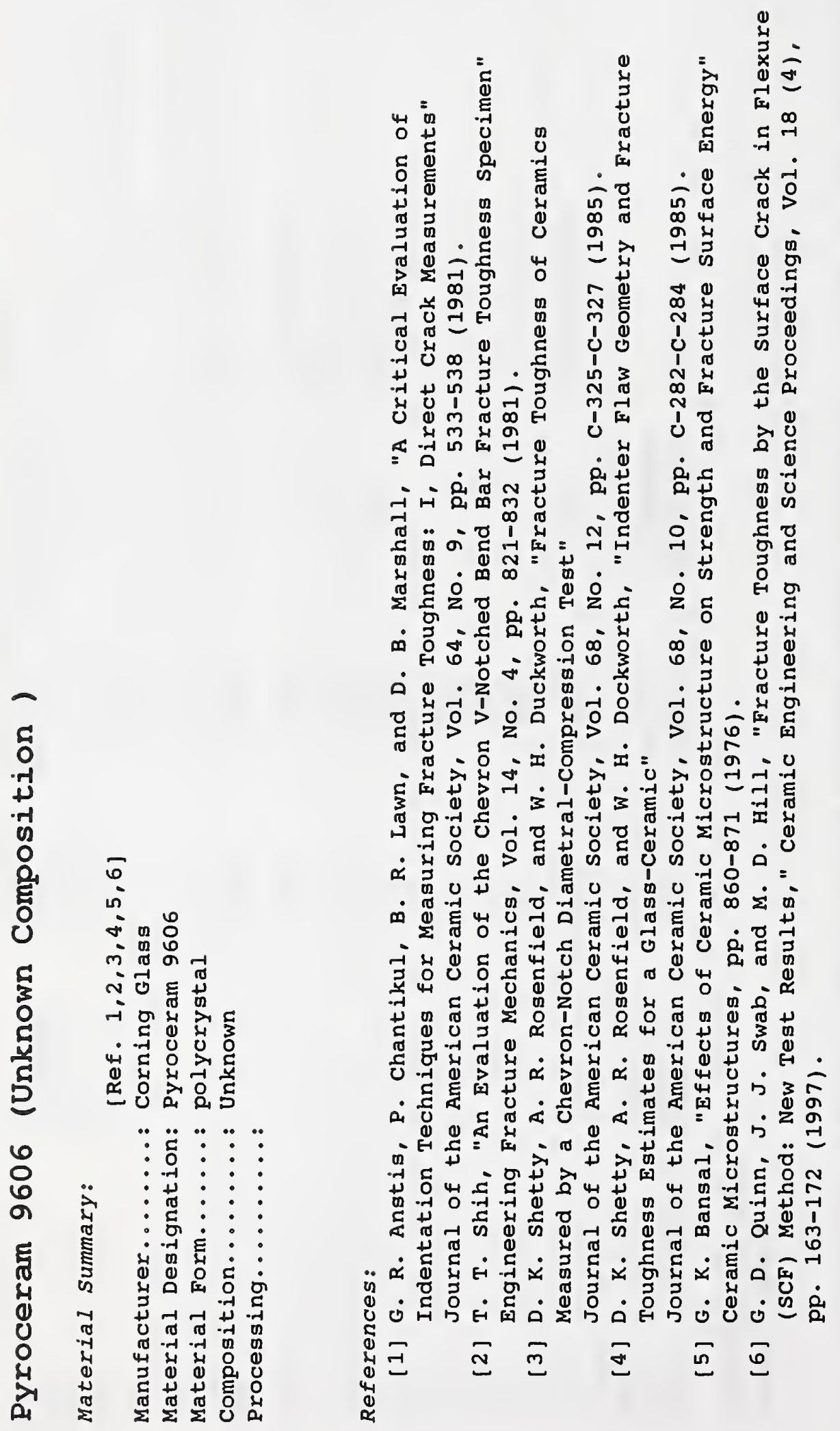




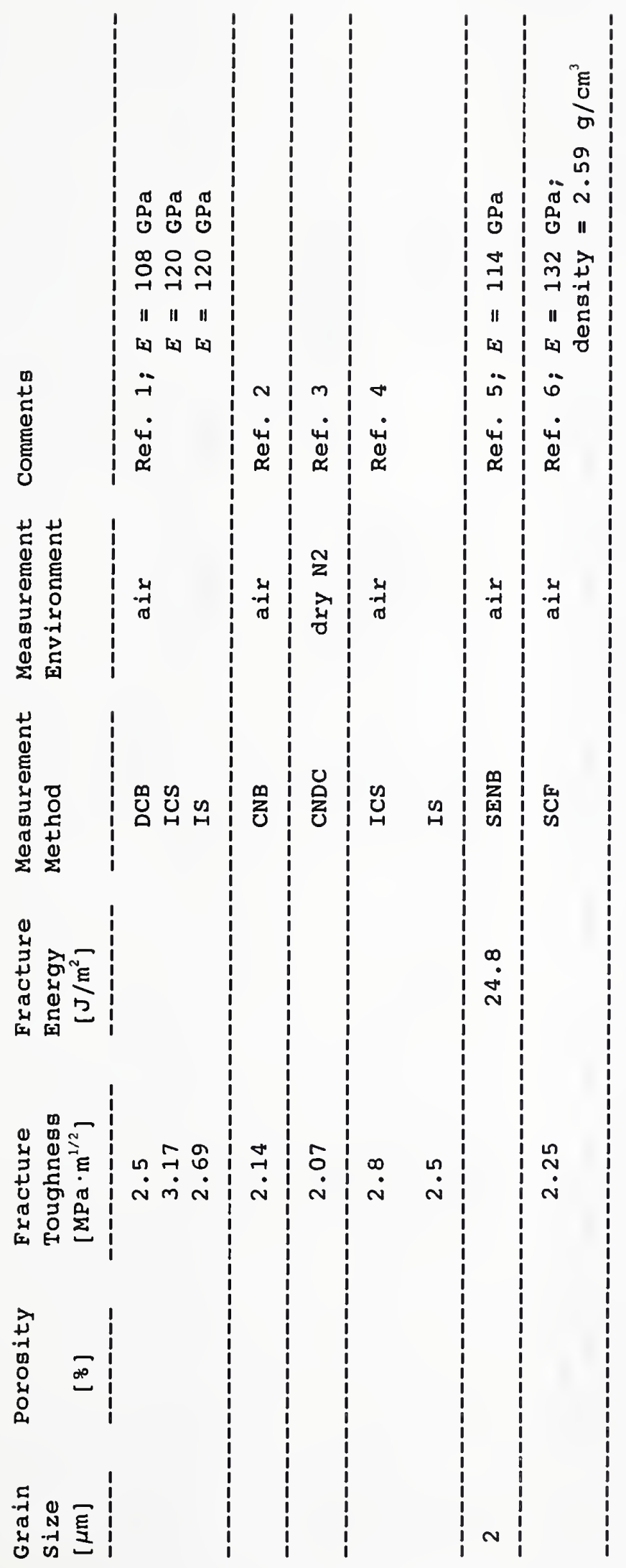




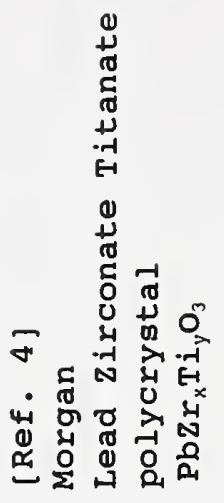

E
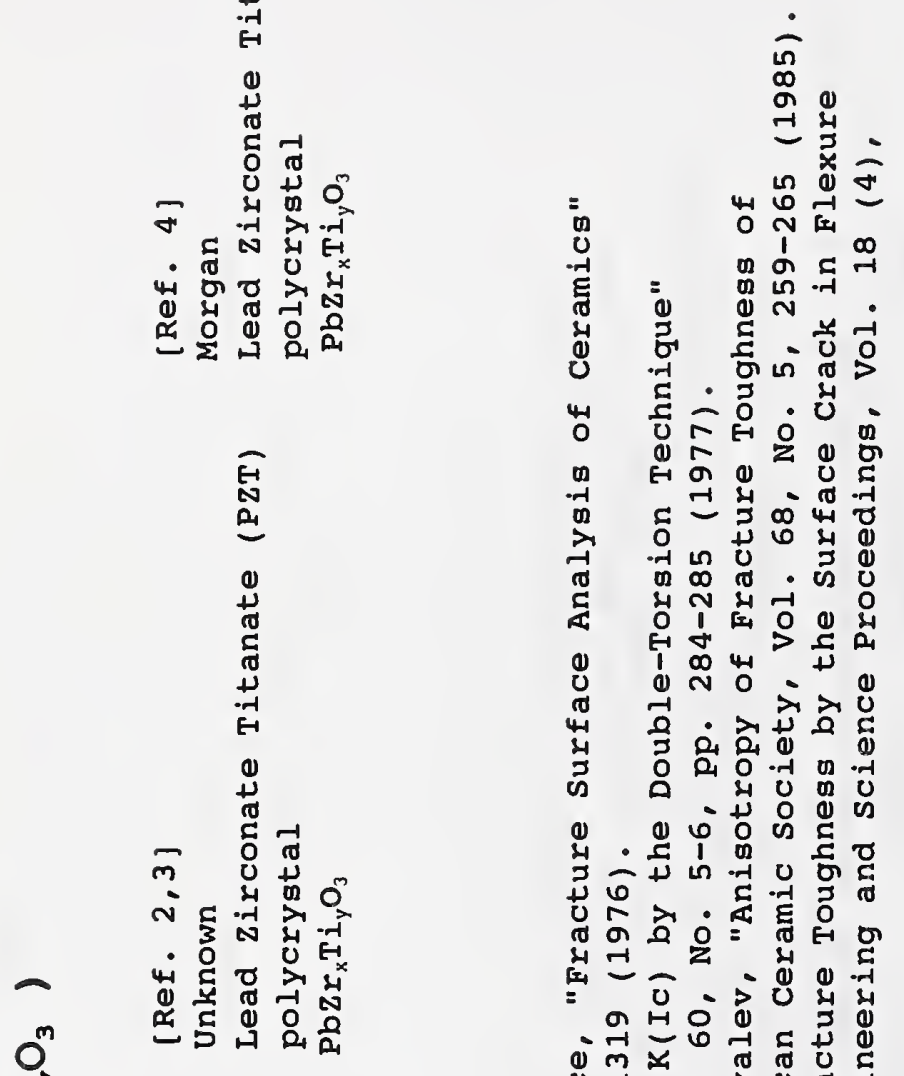

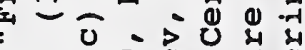
음 表 ! उ年

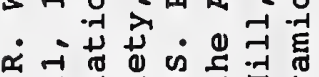

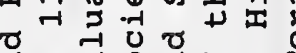
¿ 分备心 $\varepsilon^{>}=0$ o

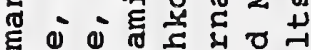
- 0 造 幽

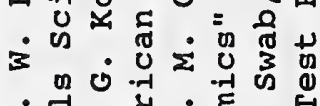

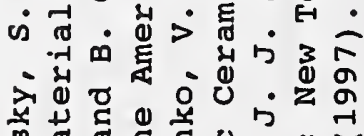

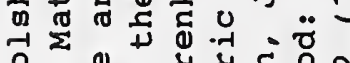
ㄴ

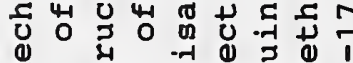
है तु

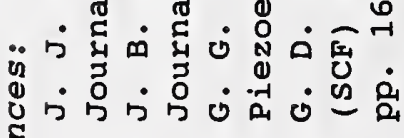

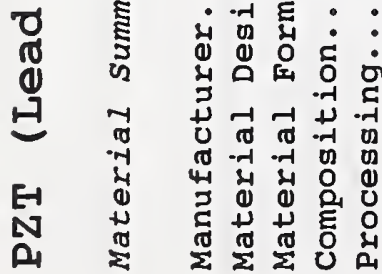
离 


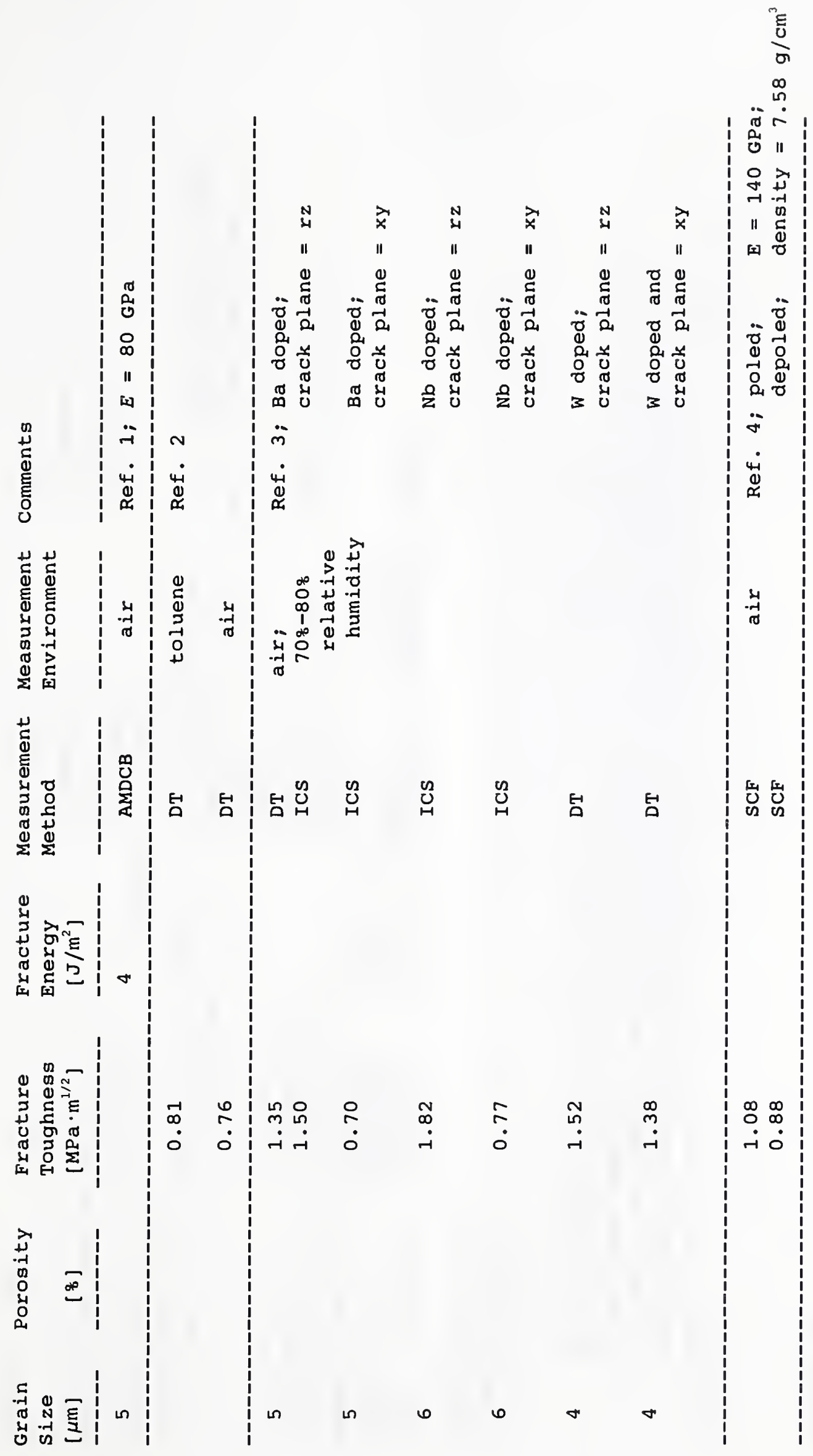



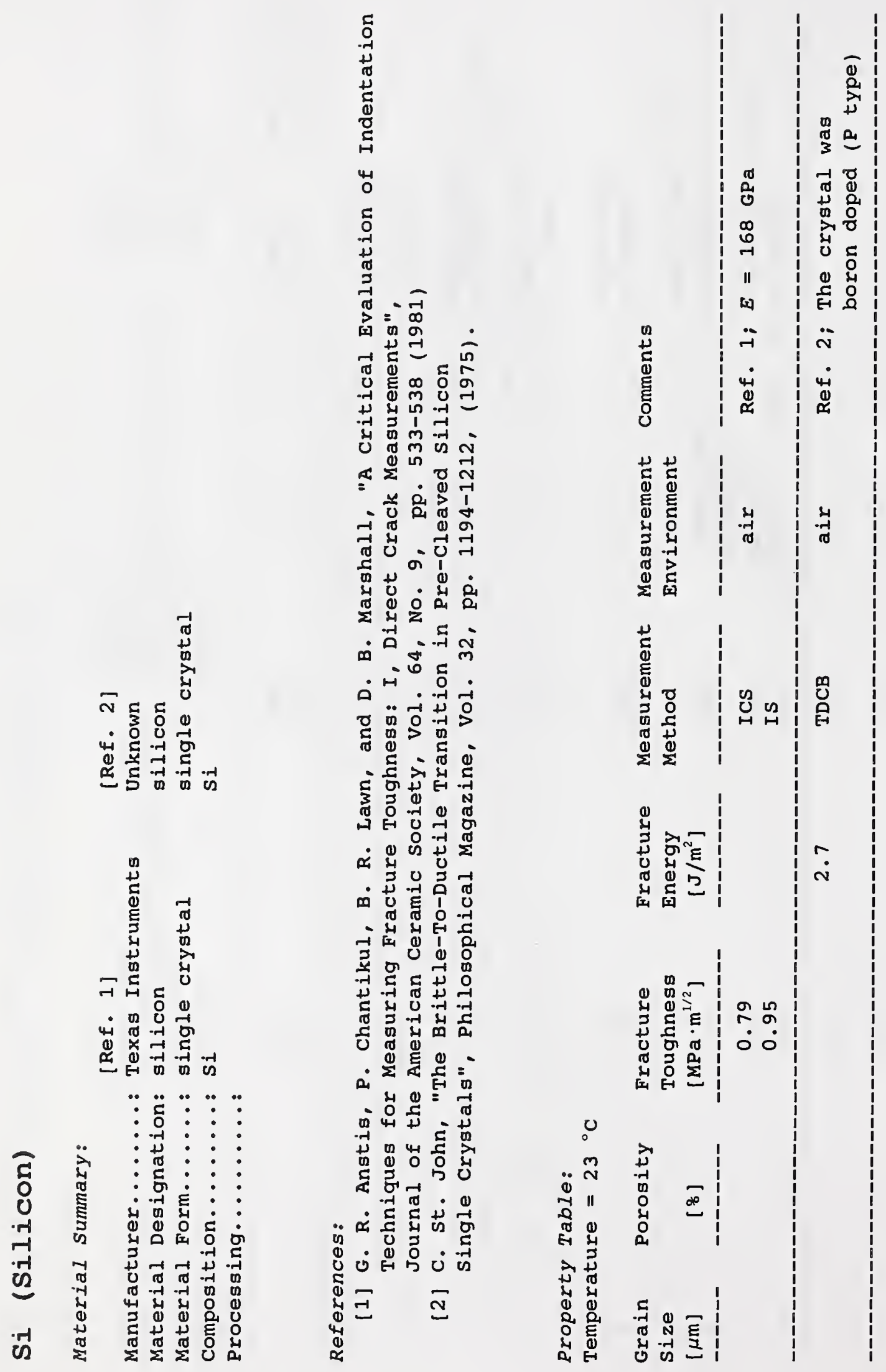


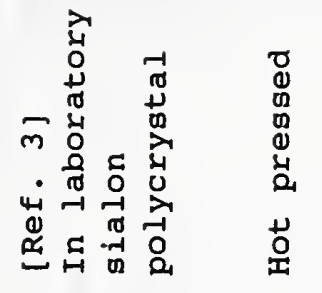

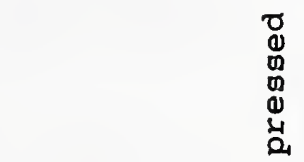
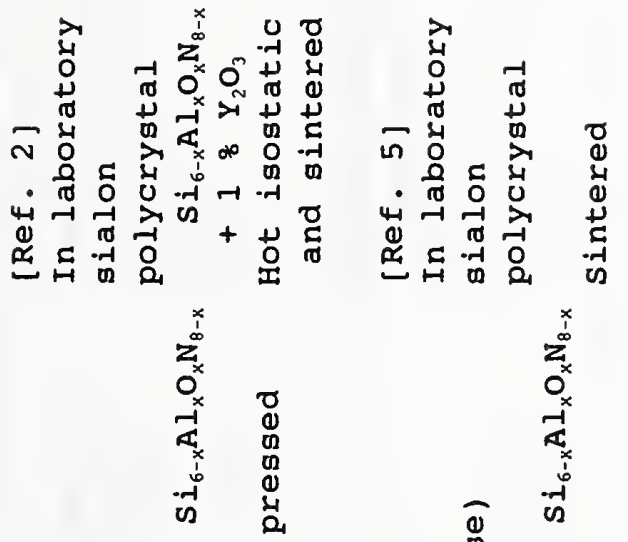

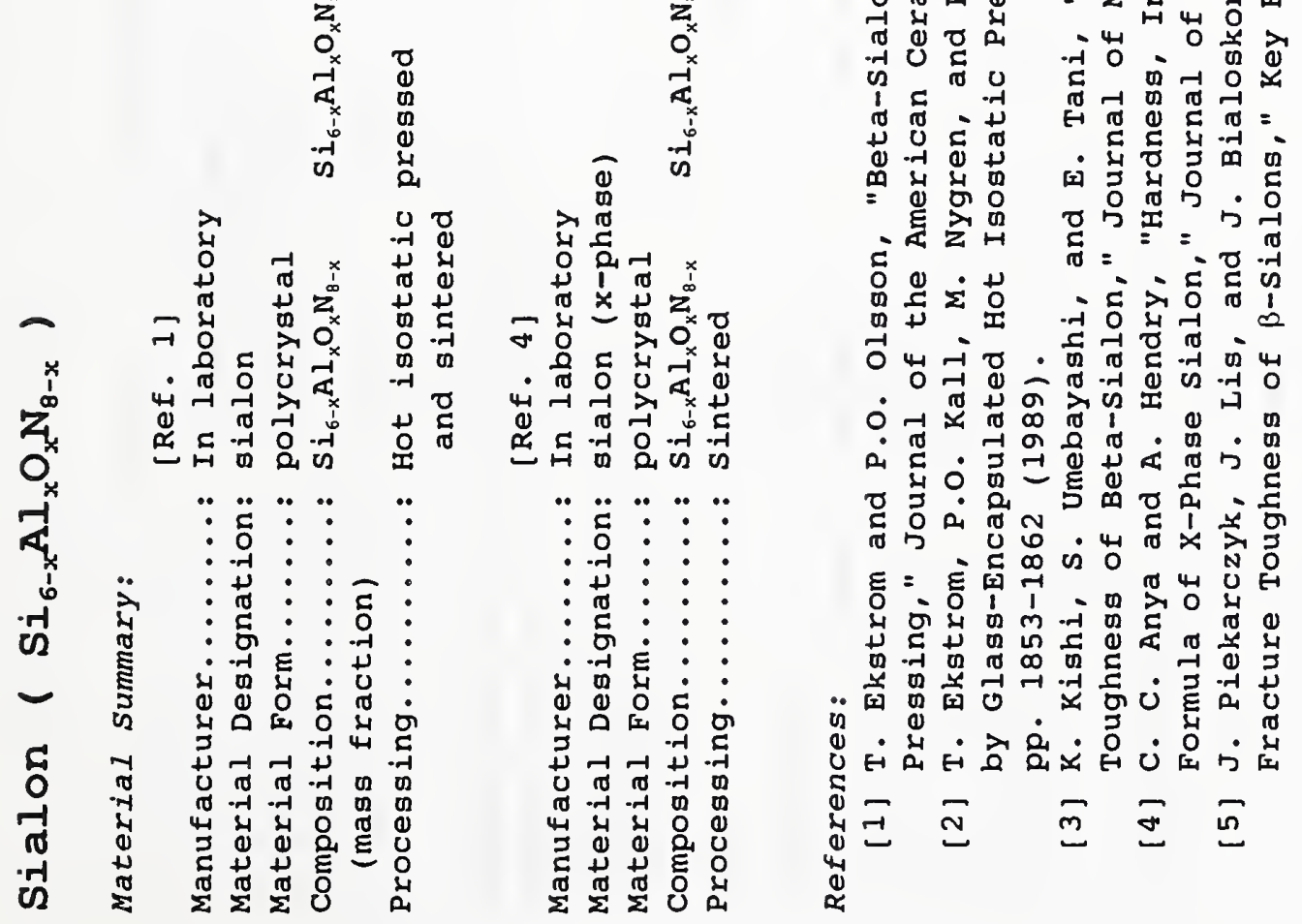

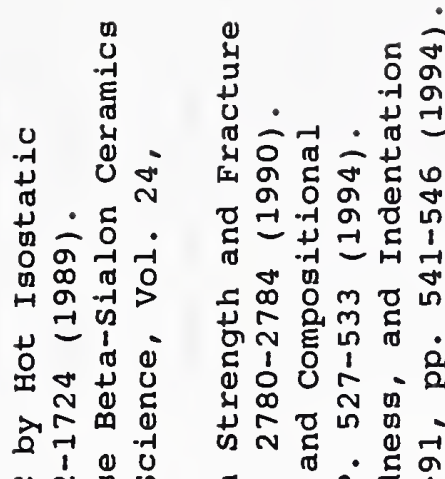

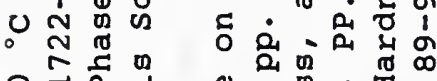

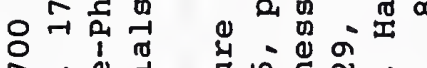

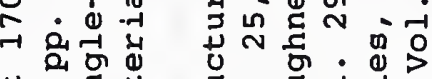
4 击

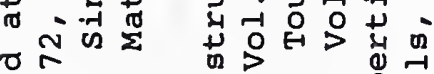

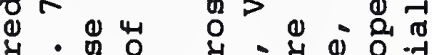
बत्व = $=5$ 出

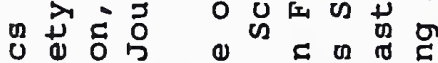

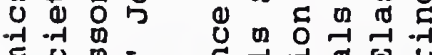
है

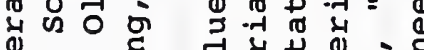

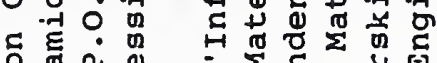

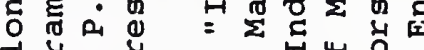

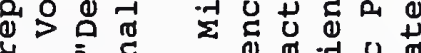
Uे 0 0. . 


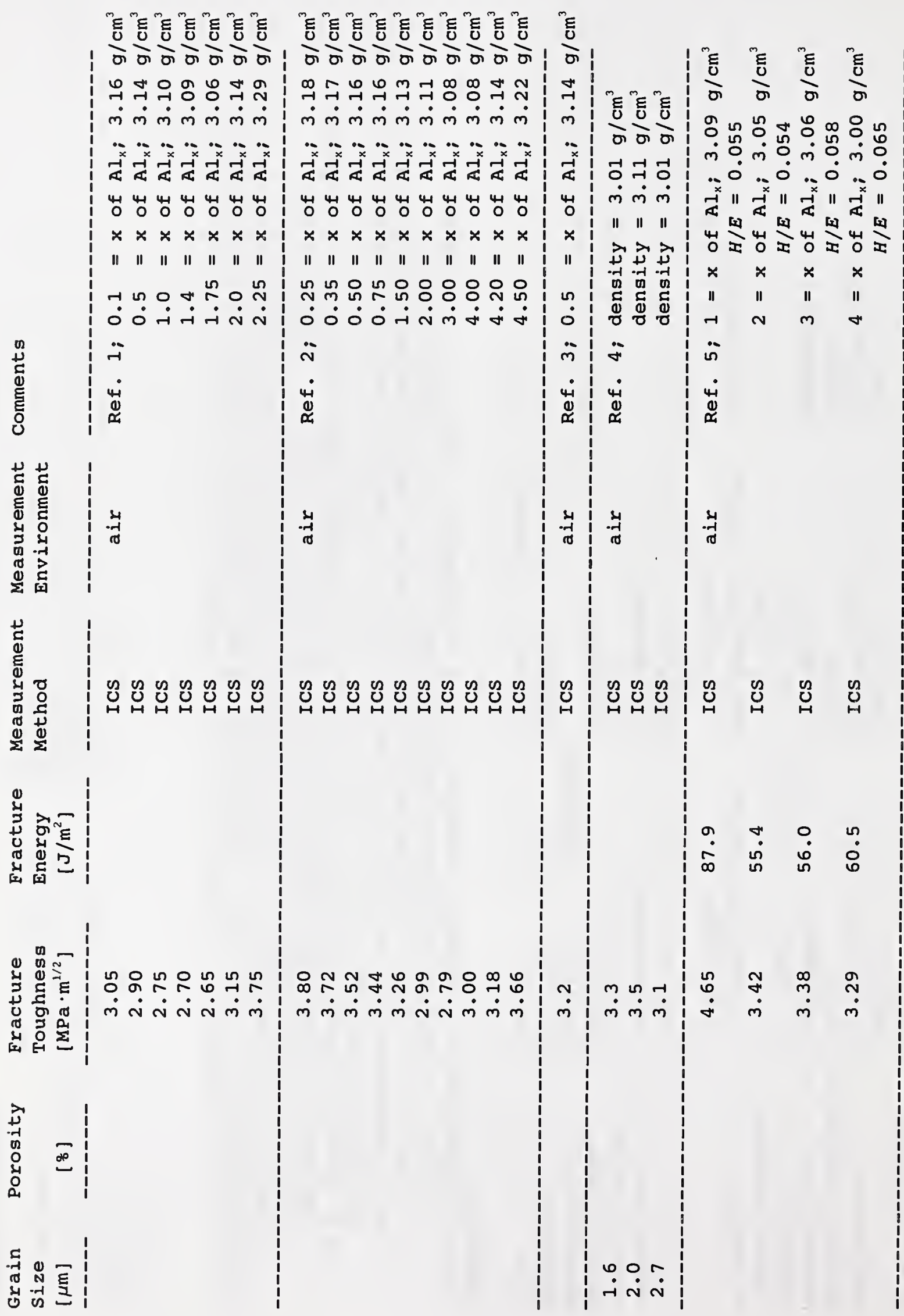



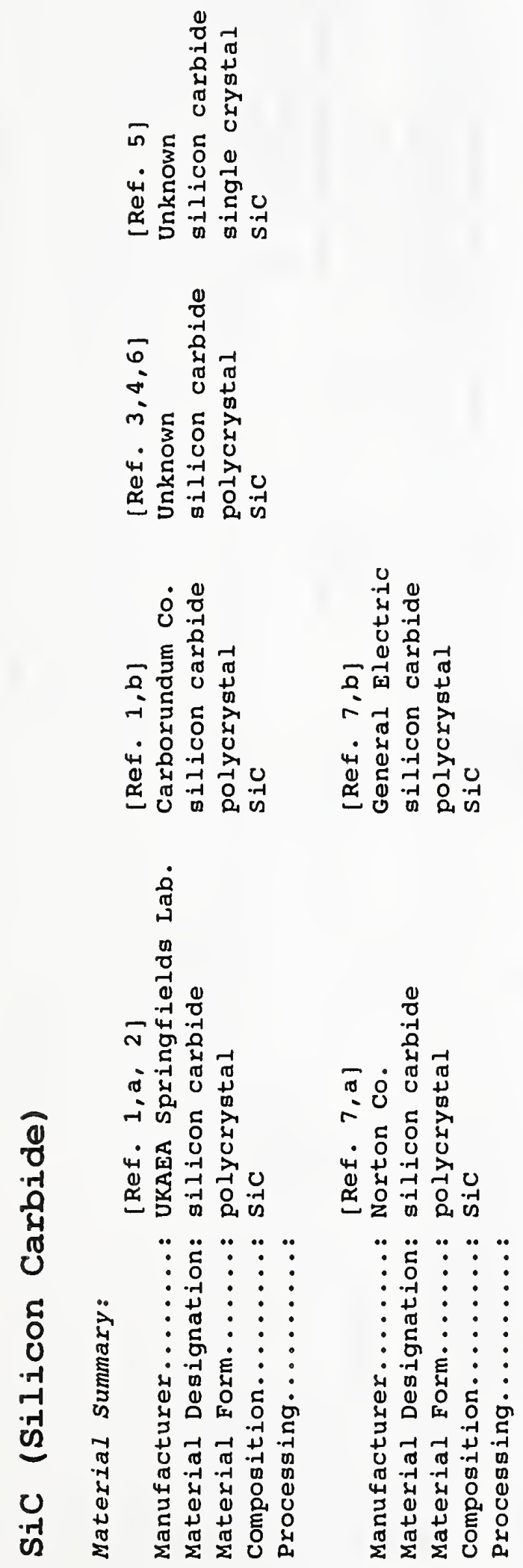

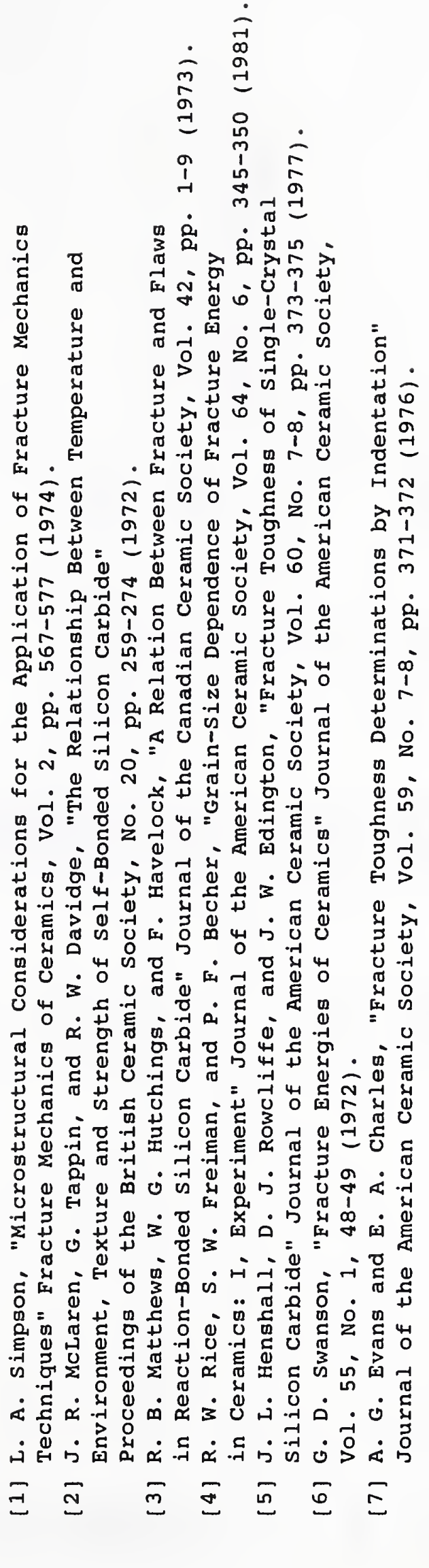




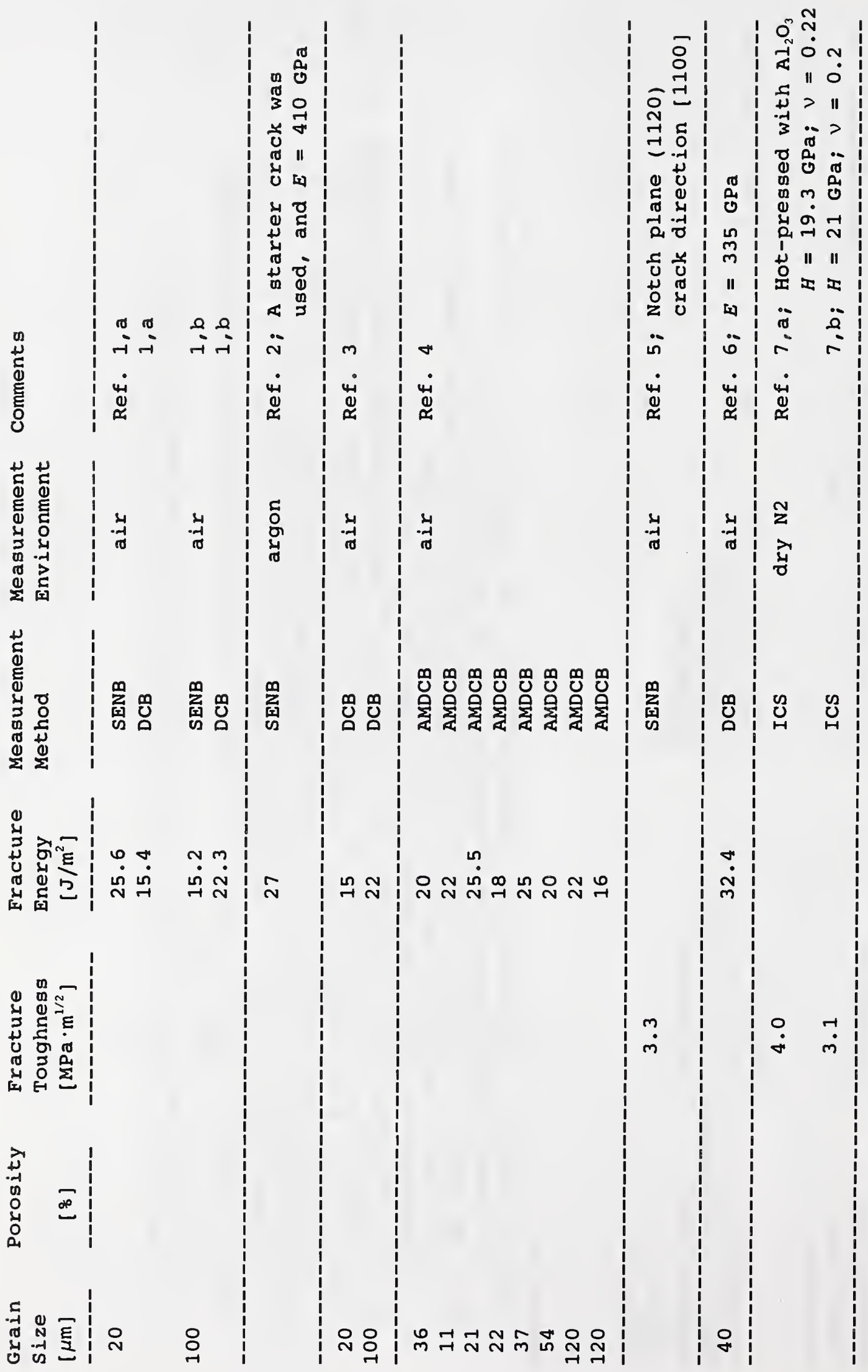



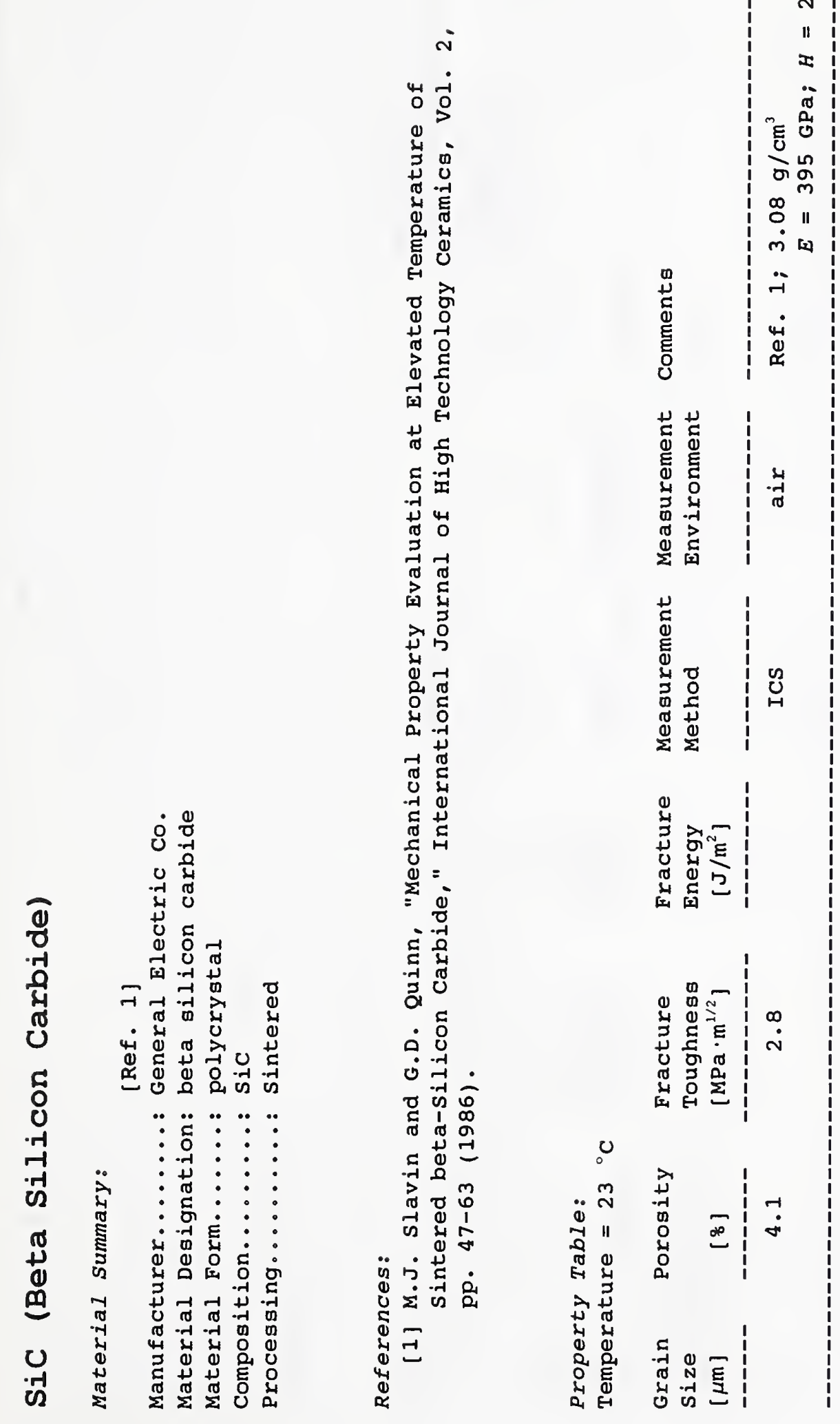


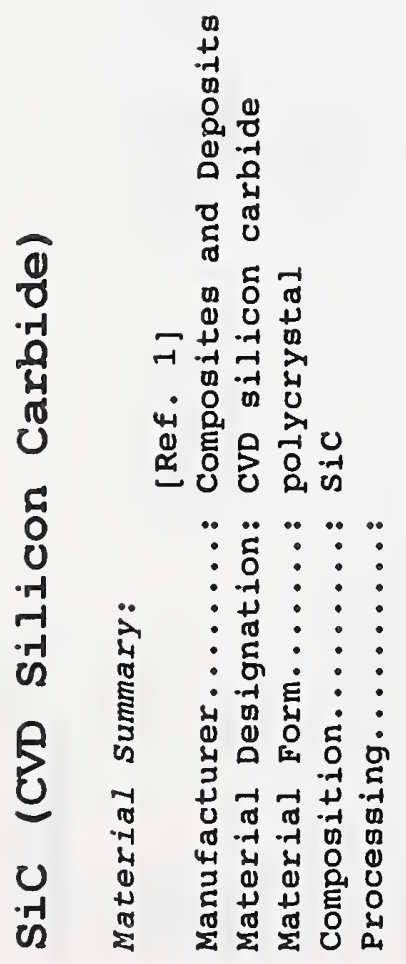

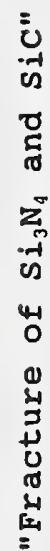

ปj

3

$\dot{\alpha}$

ซ్

客官

워

पु

这

i

$i^{\infty}$

这

㲾

-

-

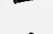

运雚

这

焉哭

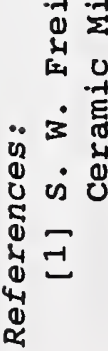
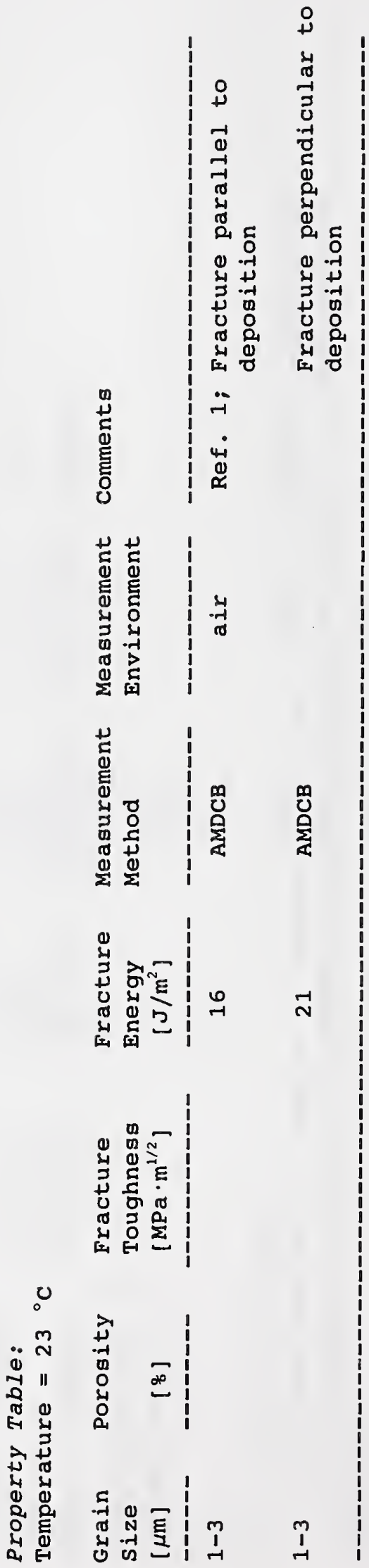


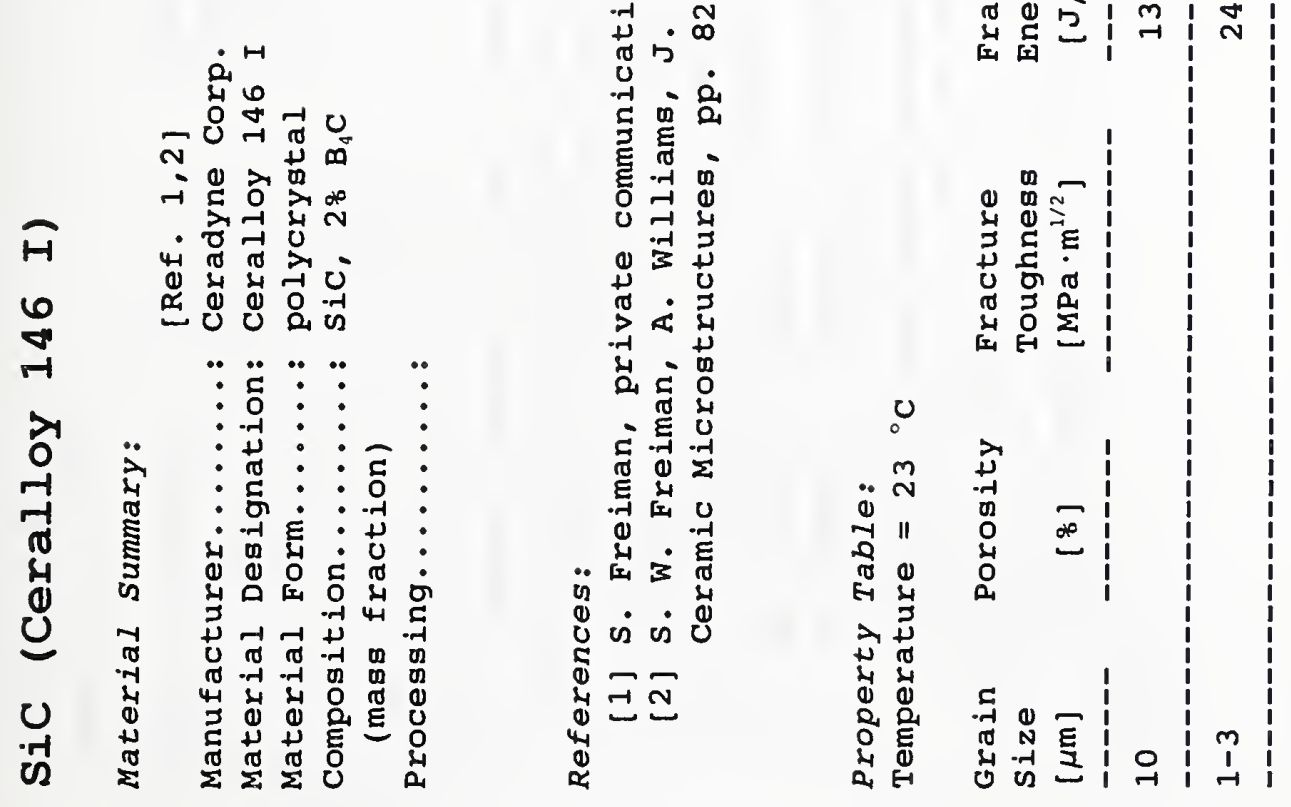


6

in

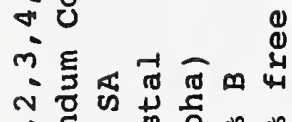

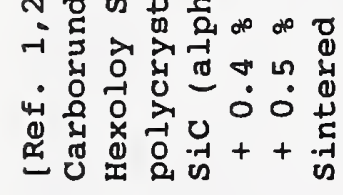

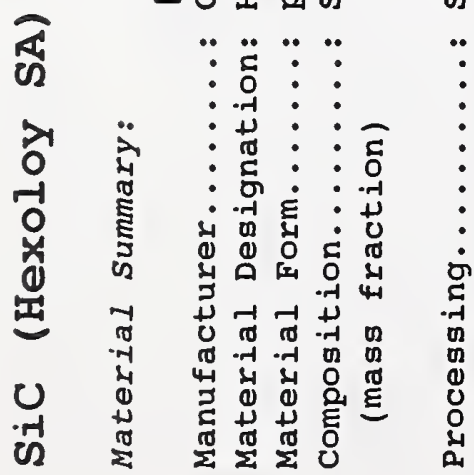

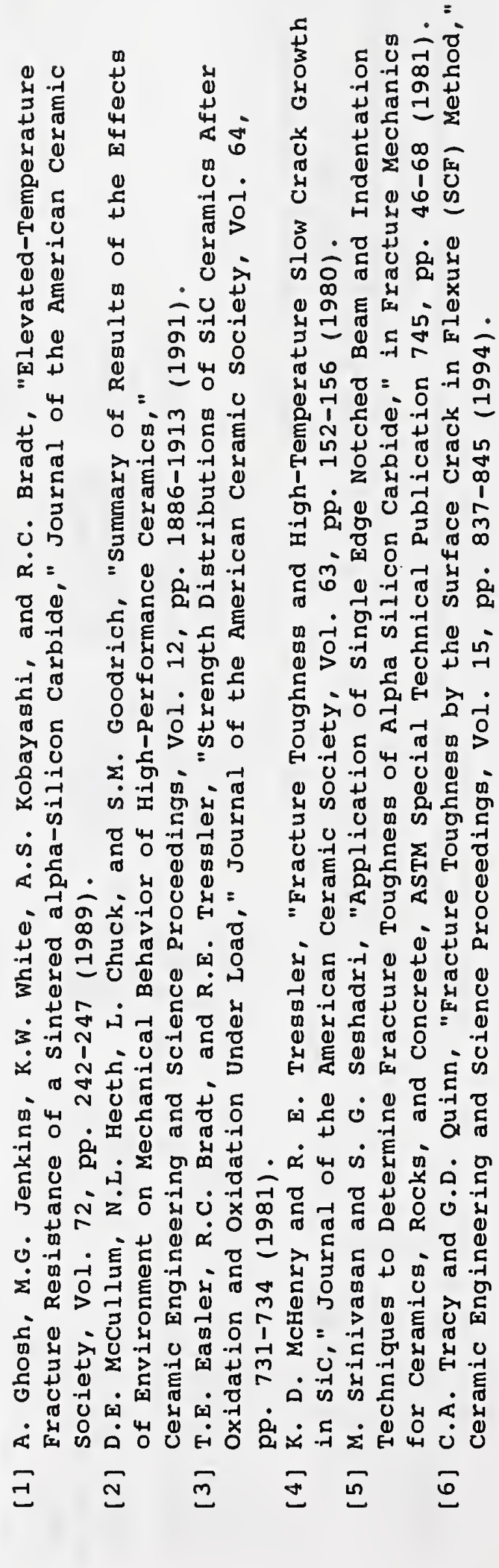




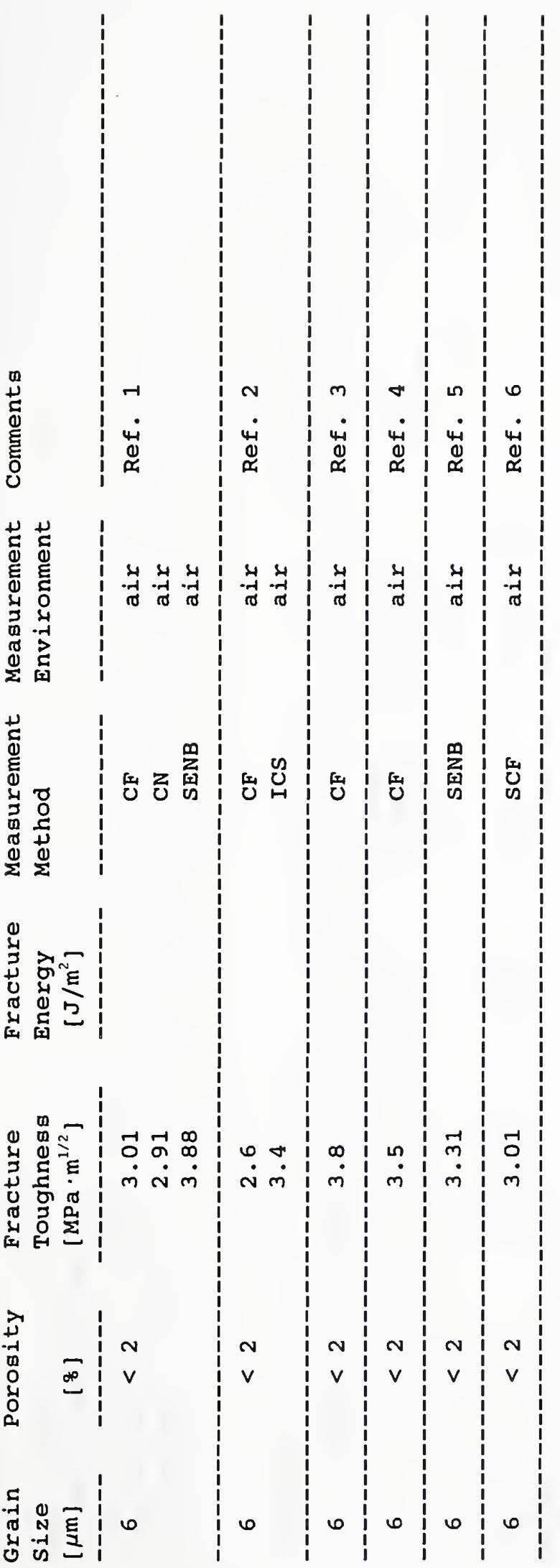



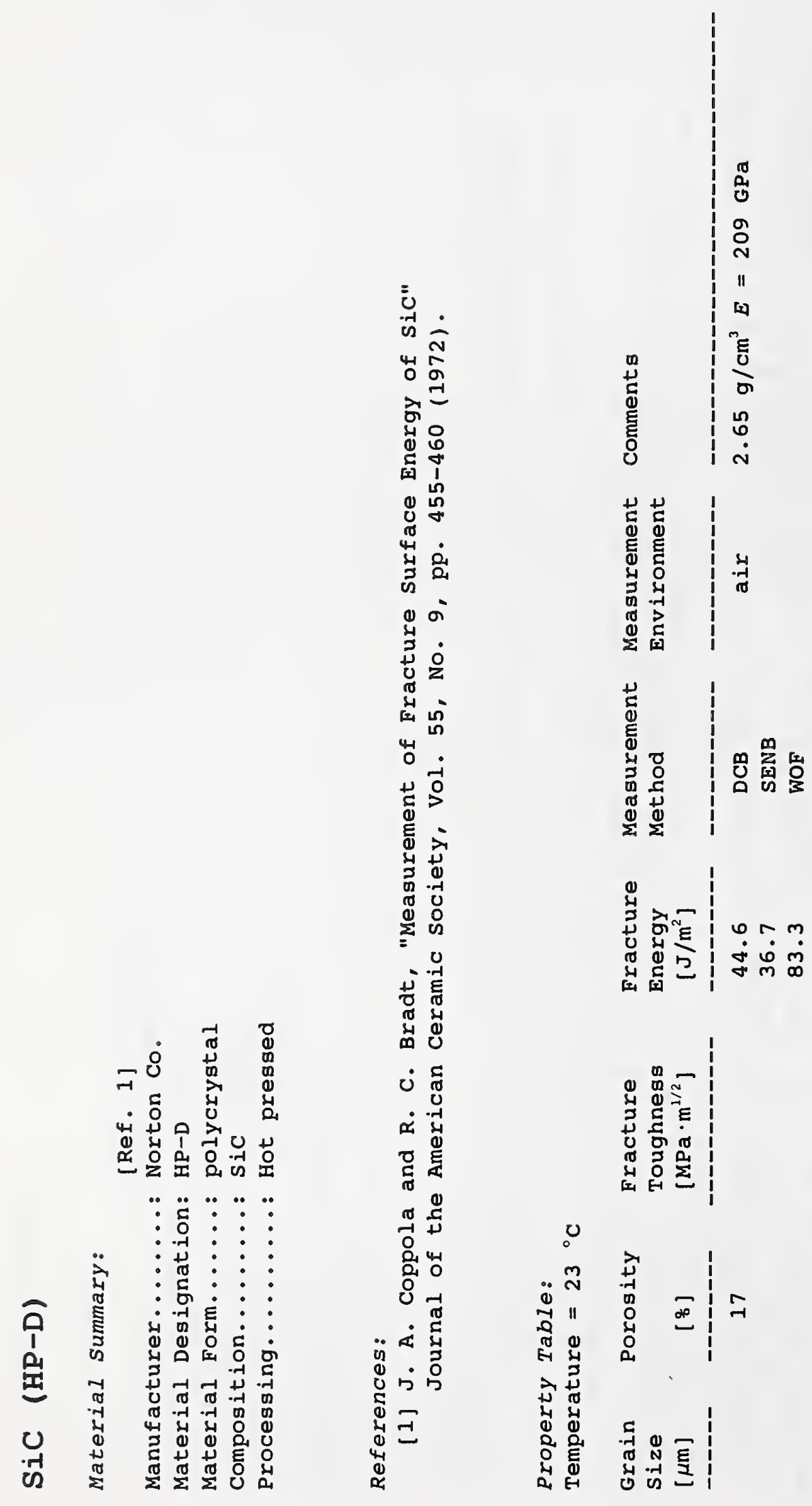


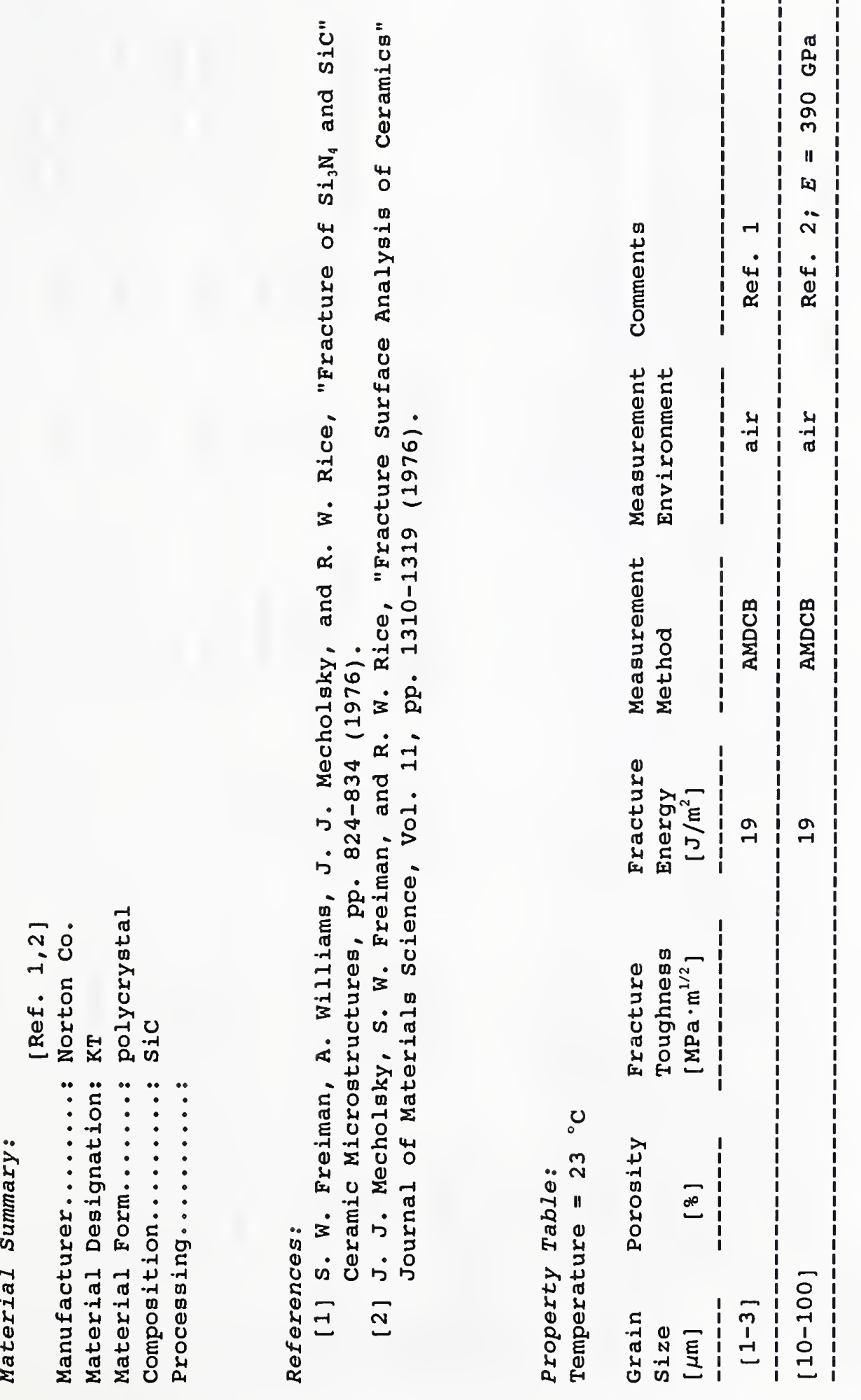



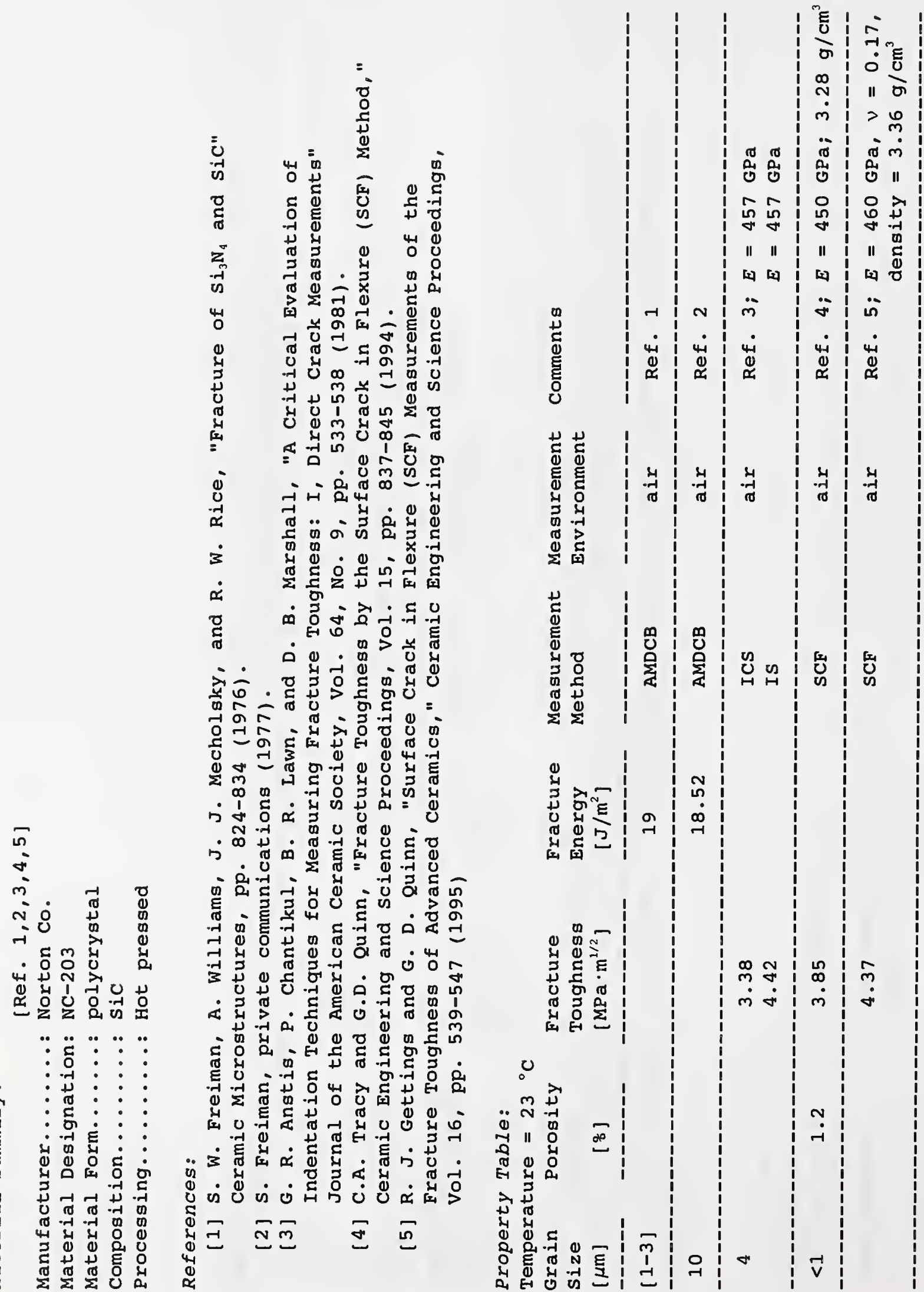

ई̇ 

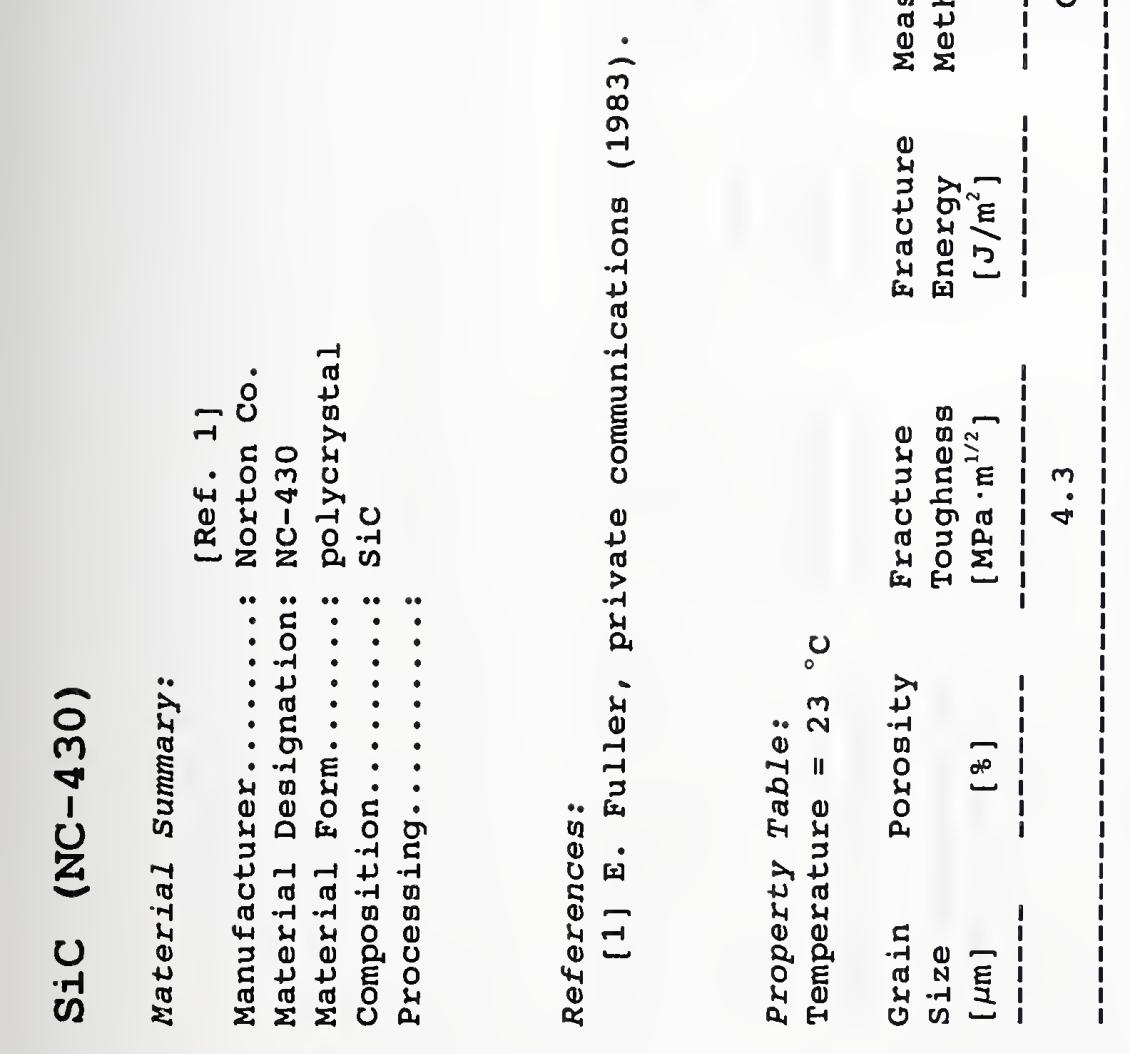


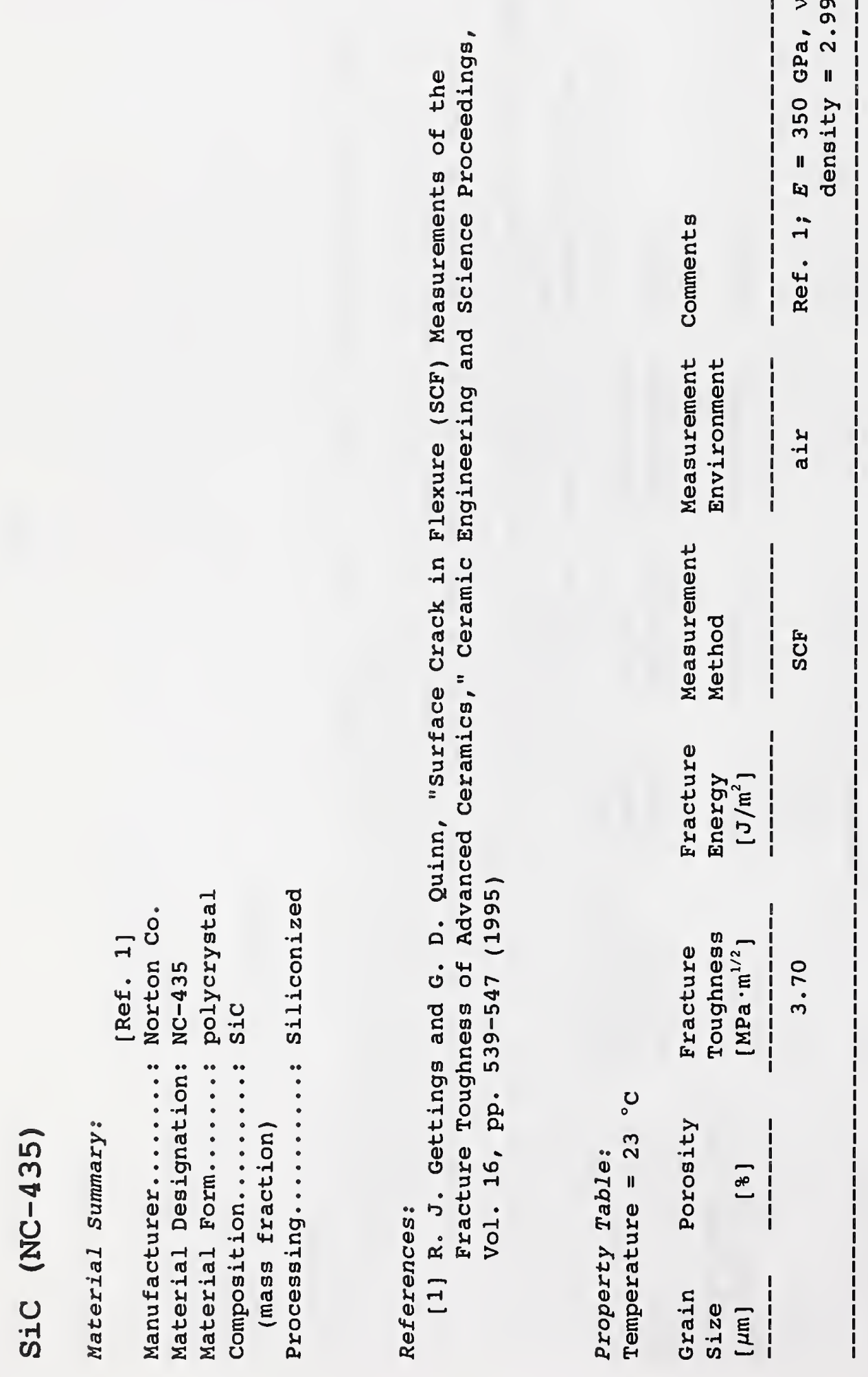



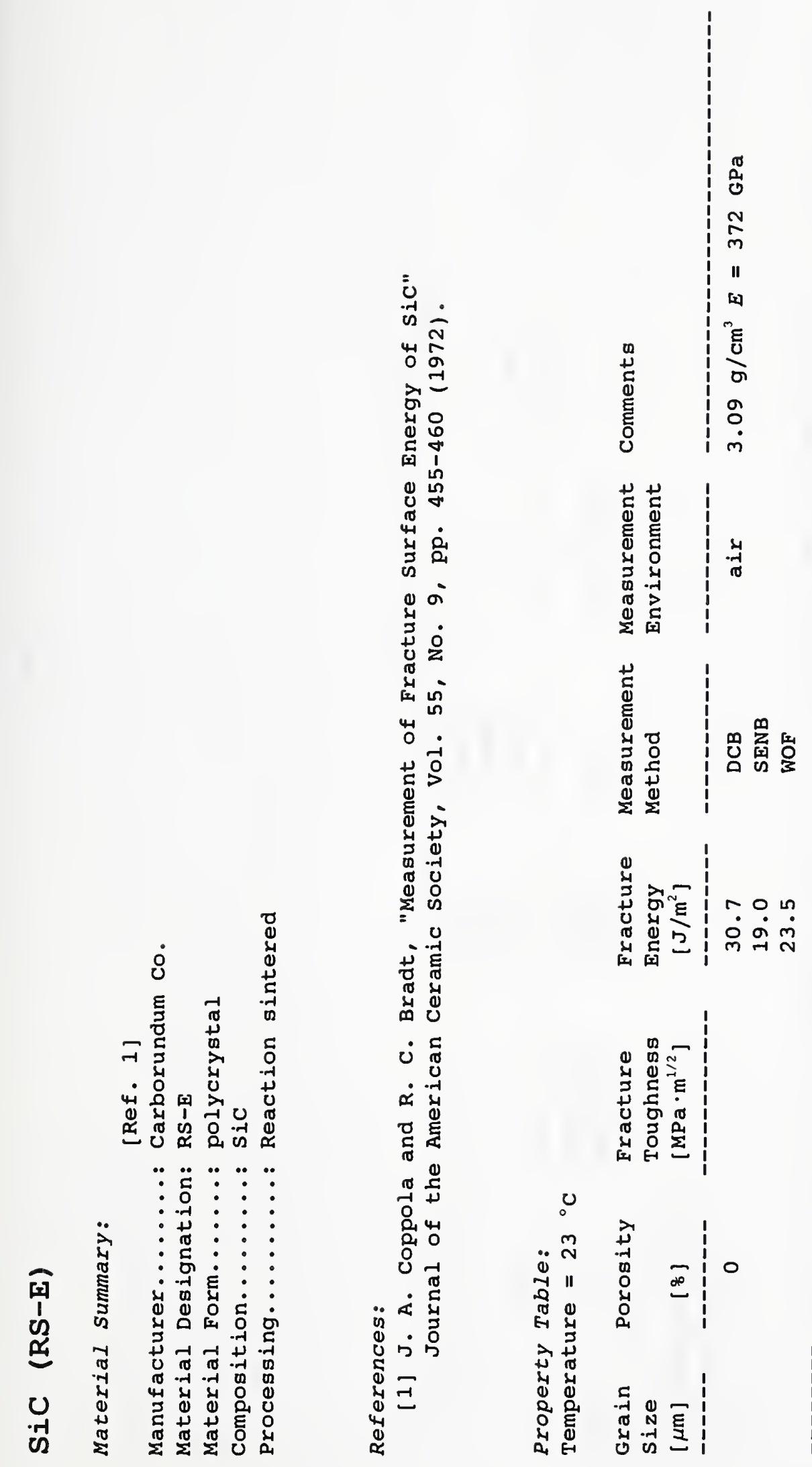


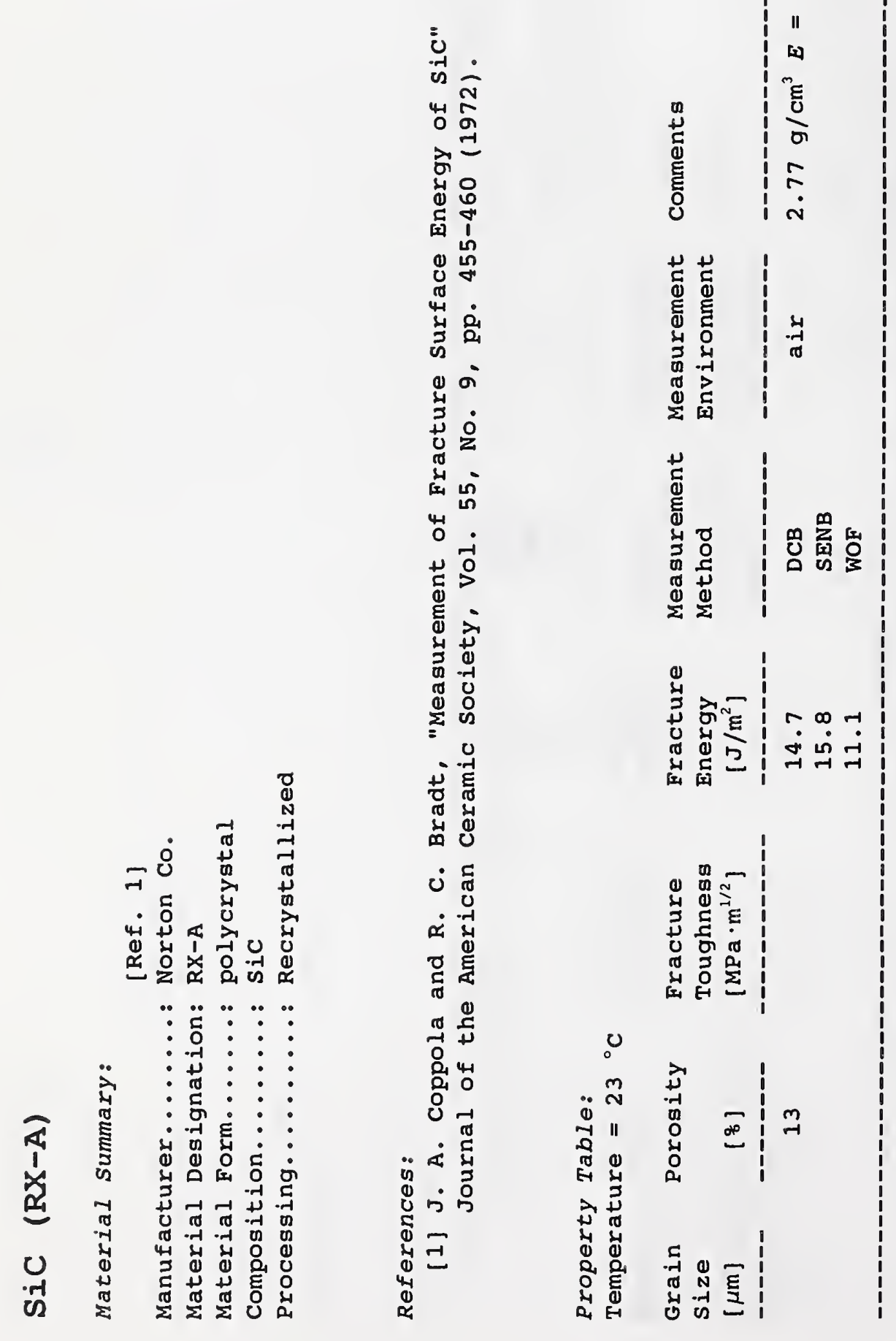

$\stackrel{1}{1}$ 


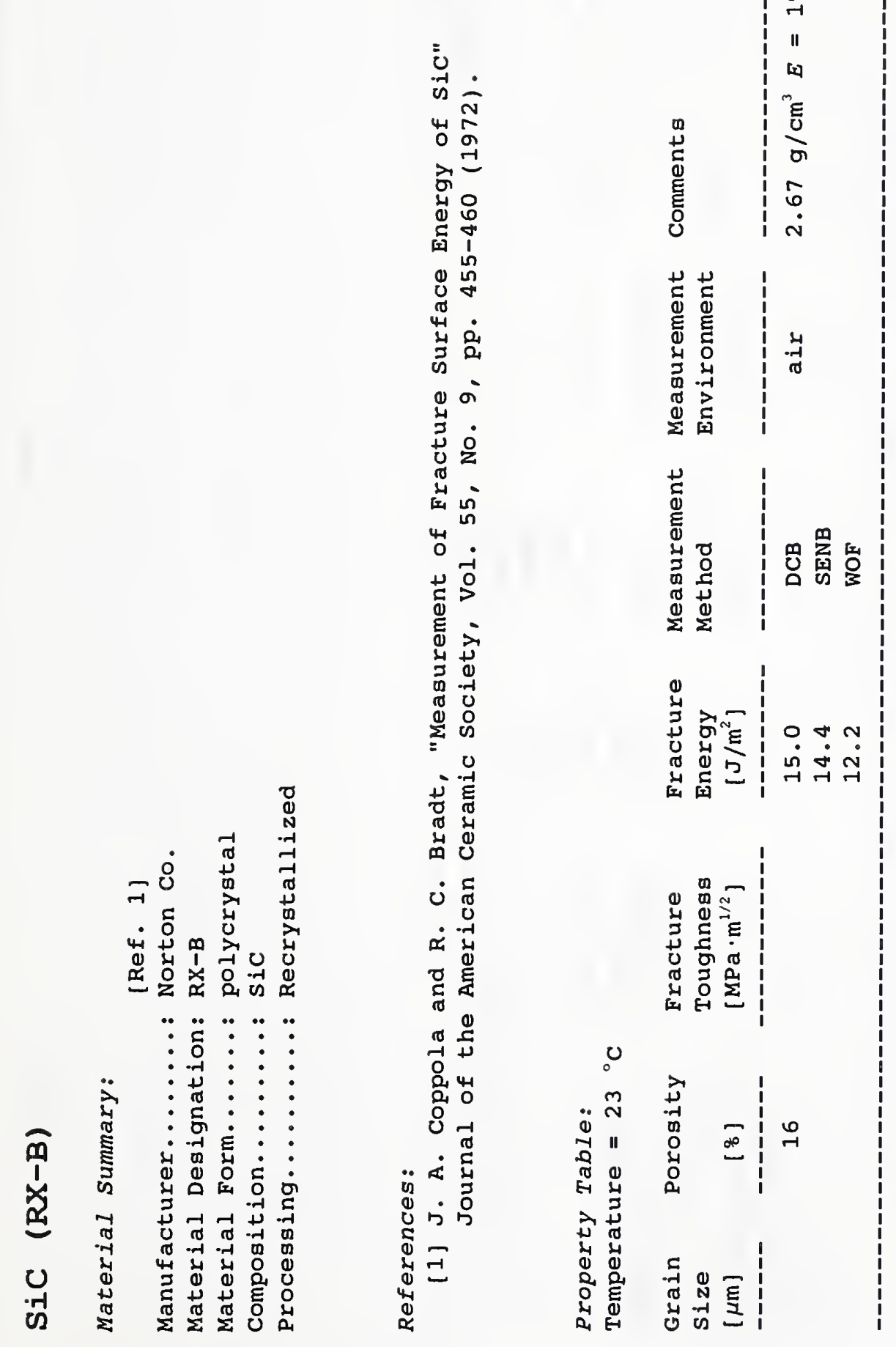




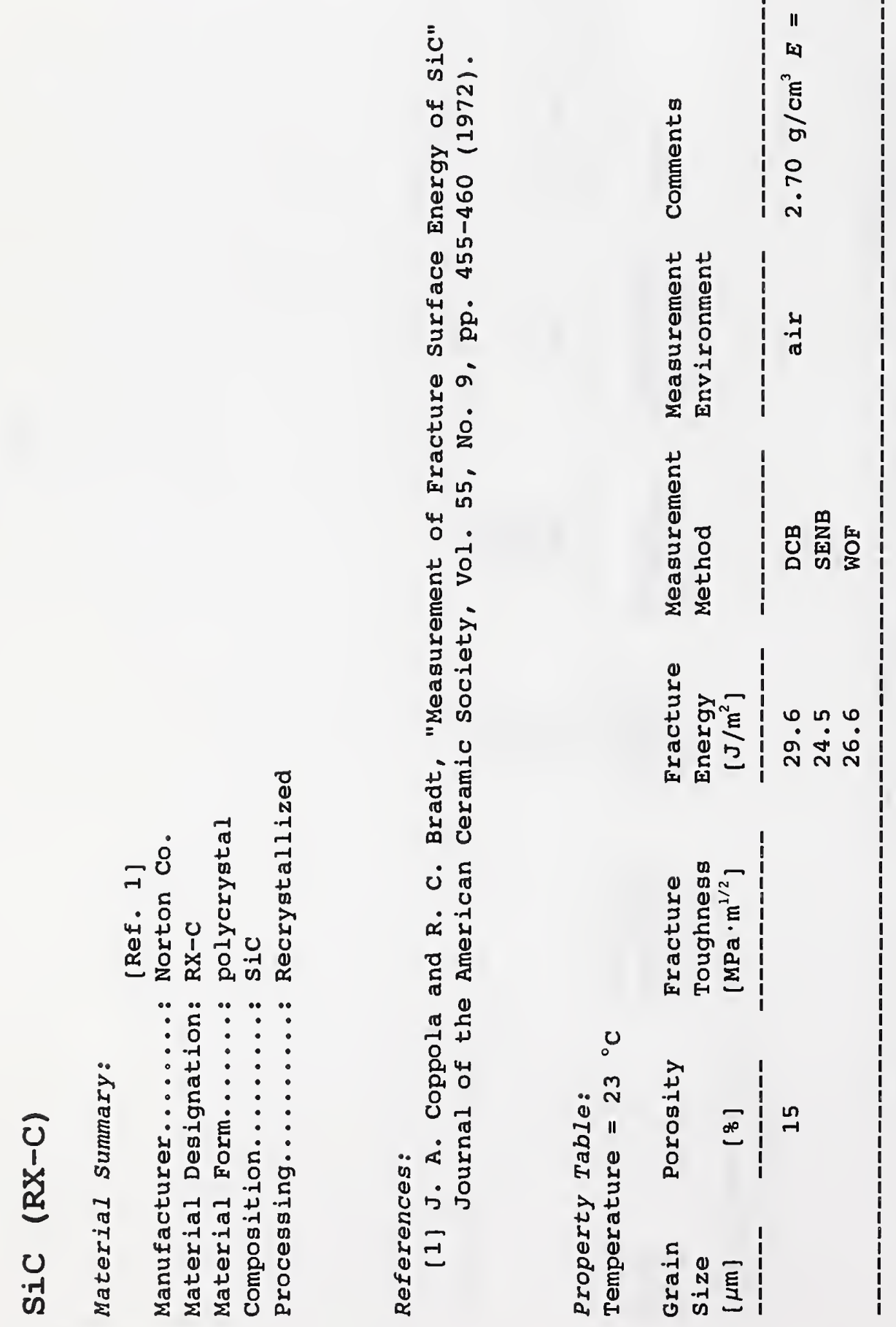




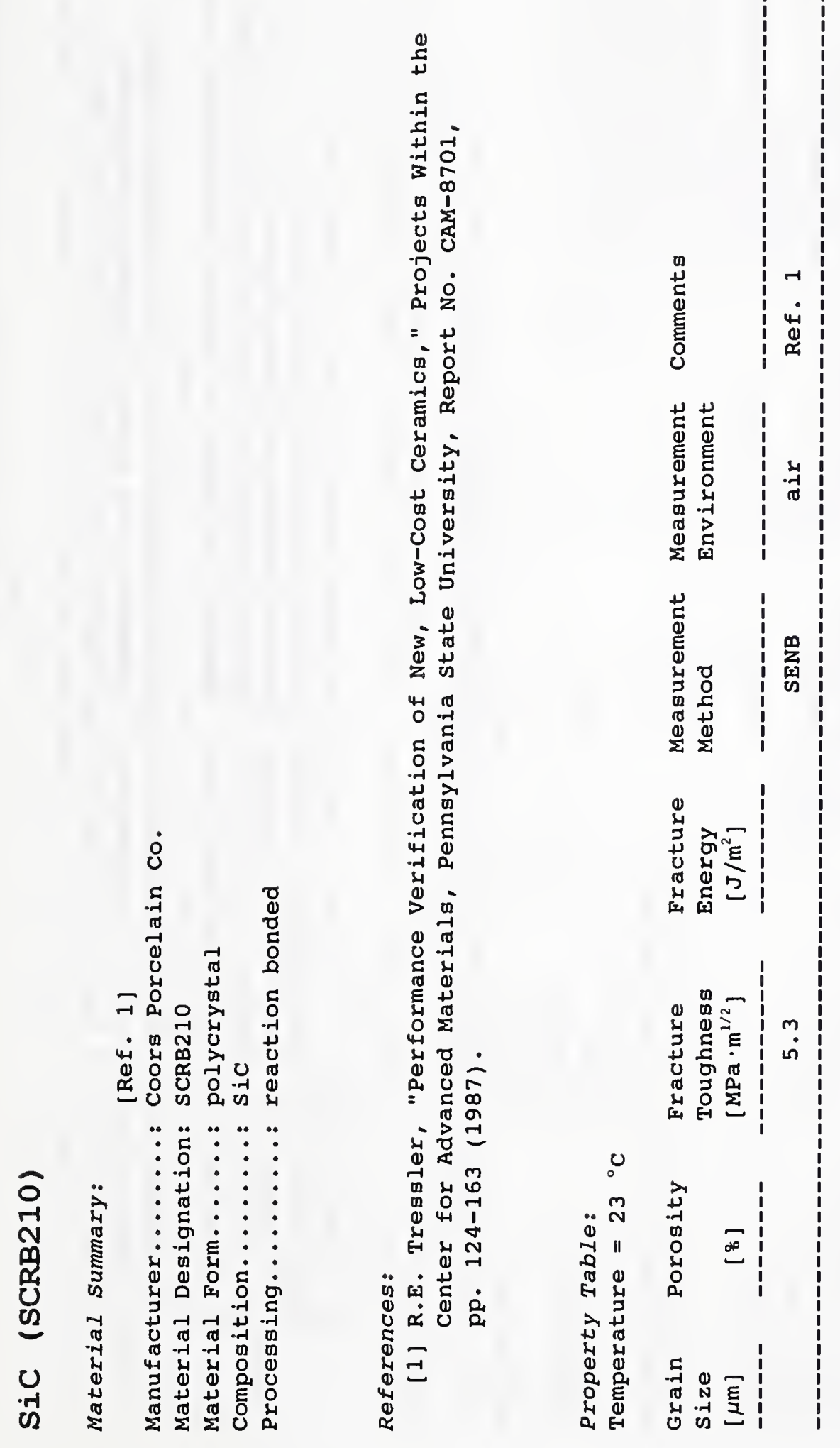

$\frac{1}{1}$ 


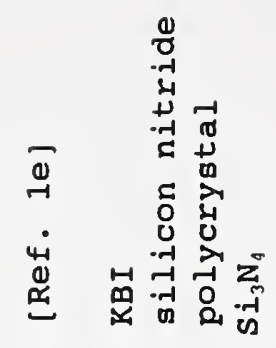

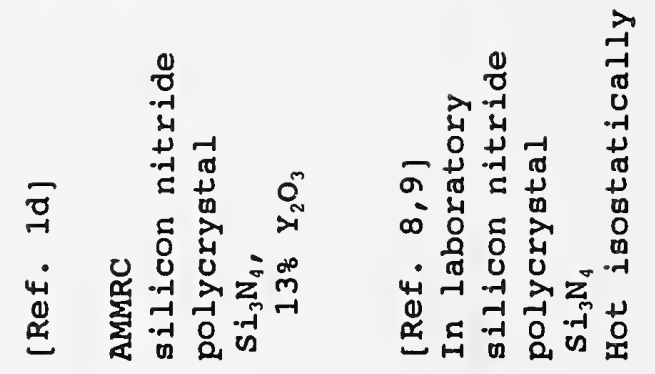

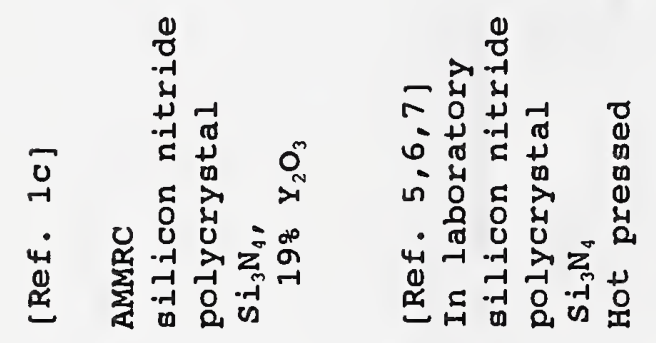

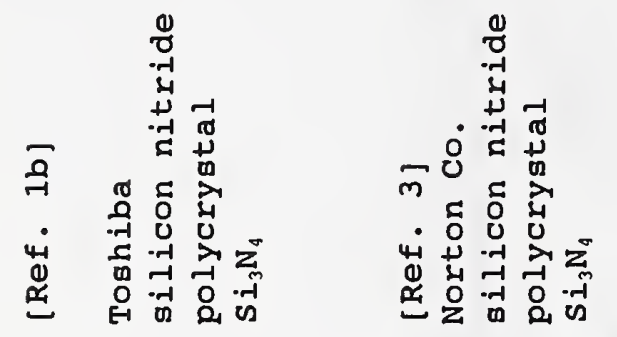

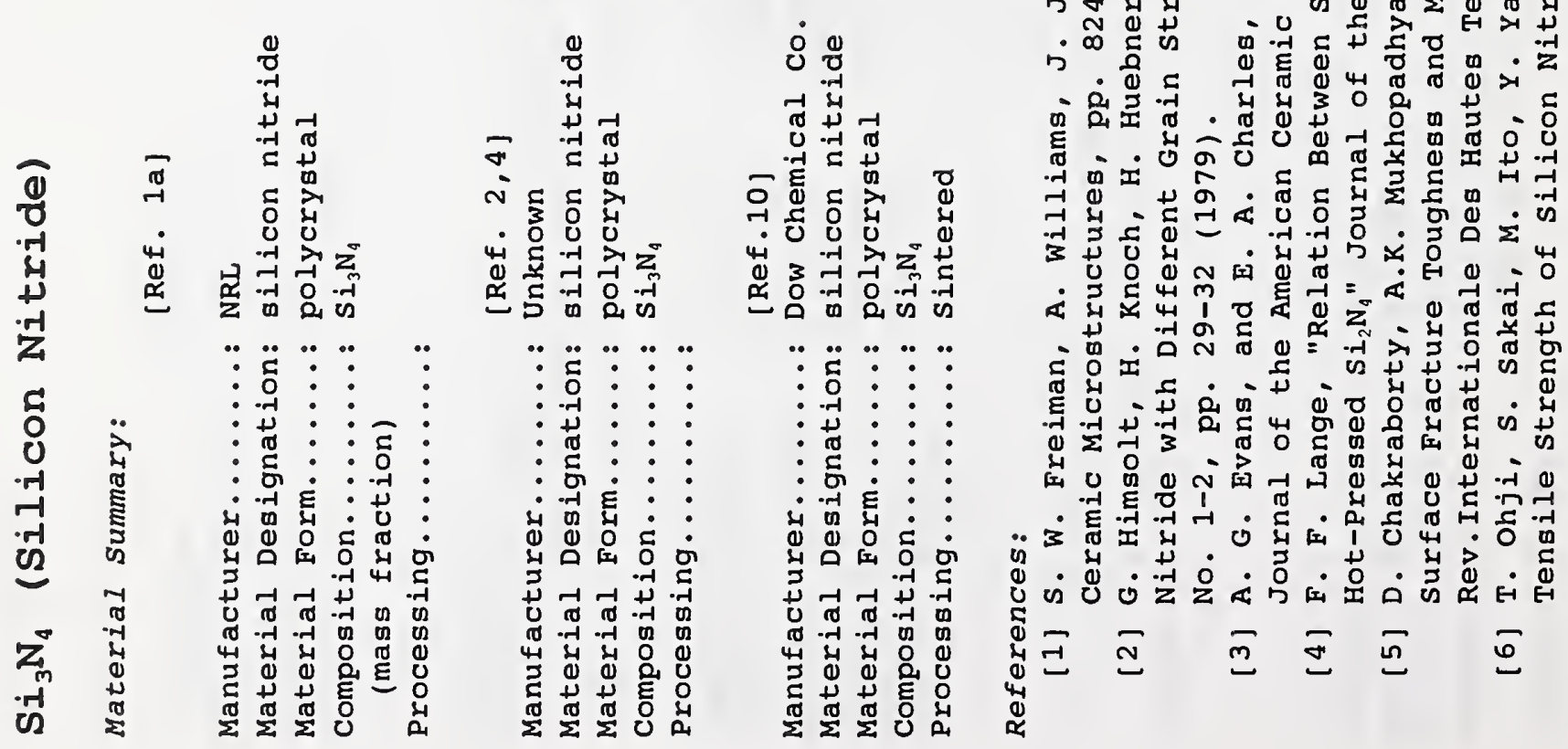

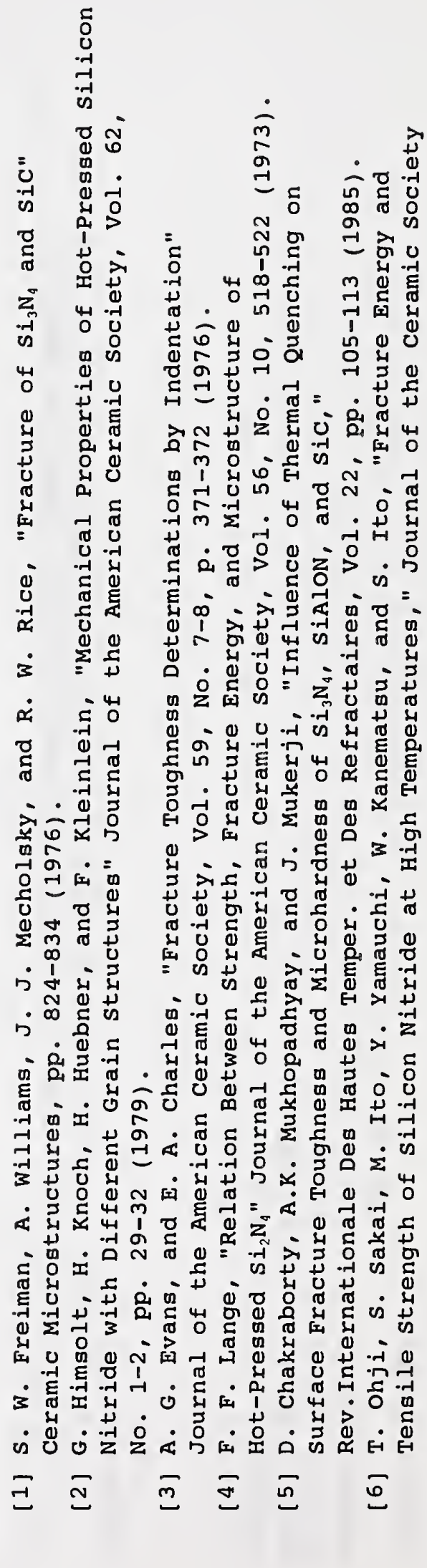




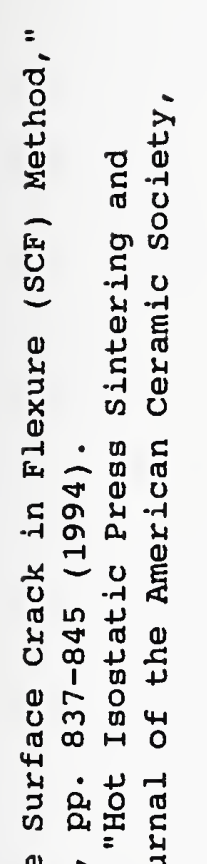

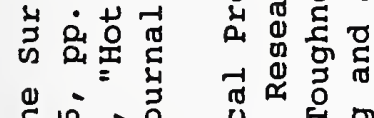
ॠ

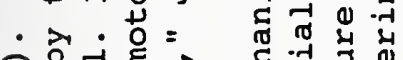
के

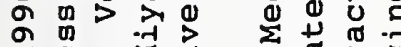

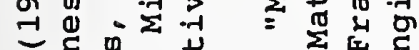

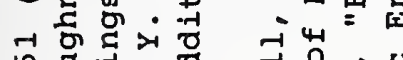
ஸै 小ू N

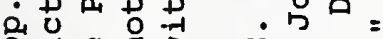

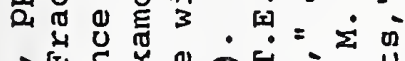

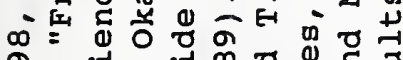
ब. 0 . 로 $>$ 苛究 :

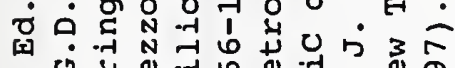

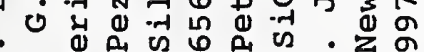
मे

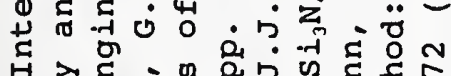

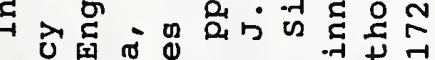

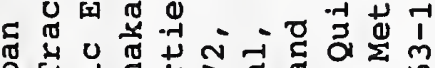
贯

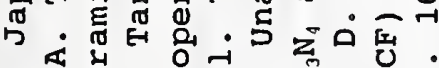
山

E $\bar{\Xi}$ 三

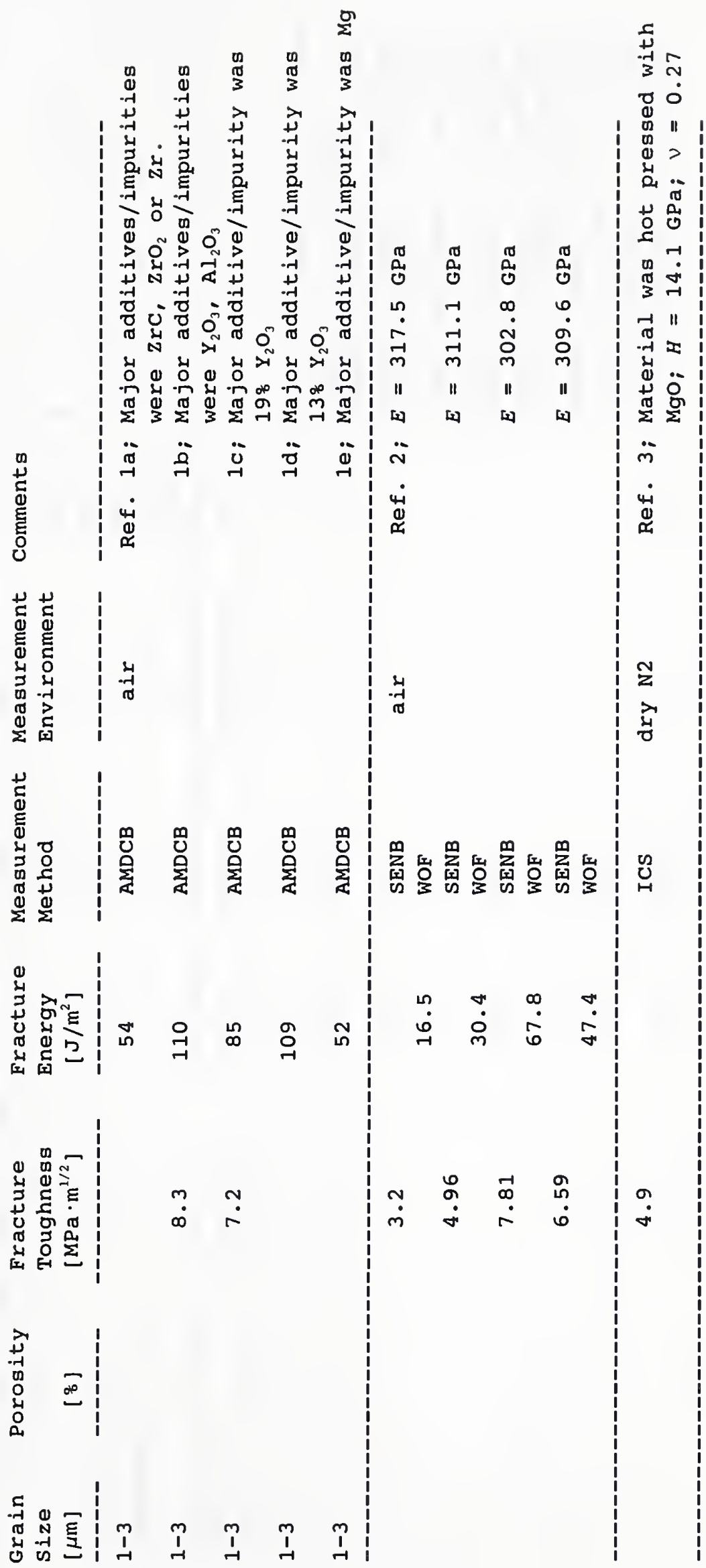




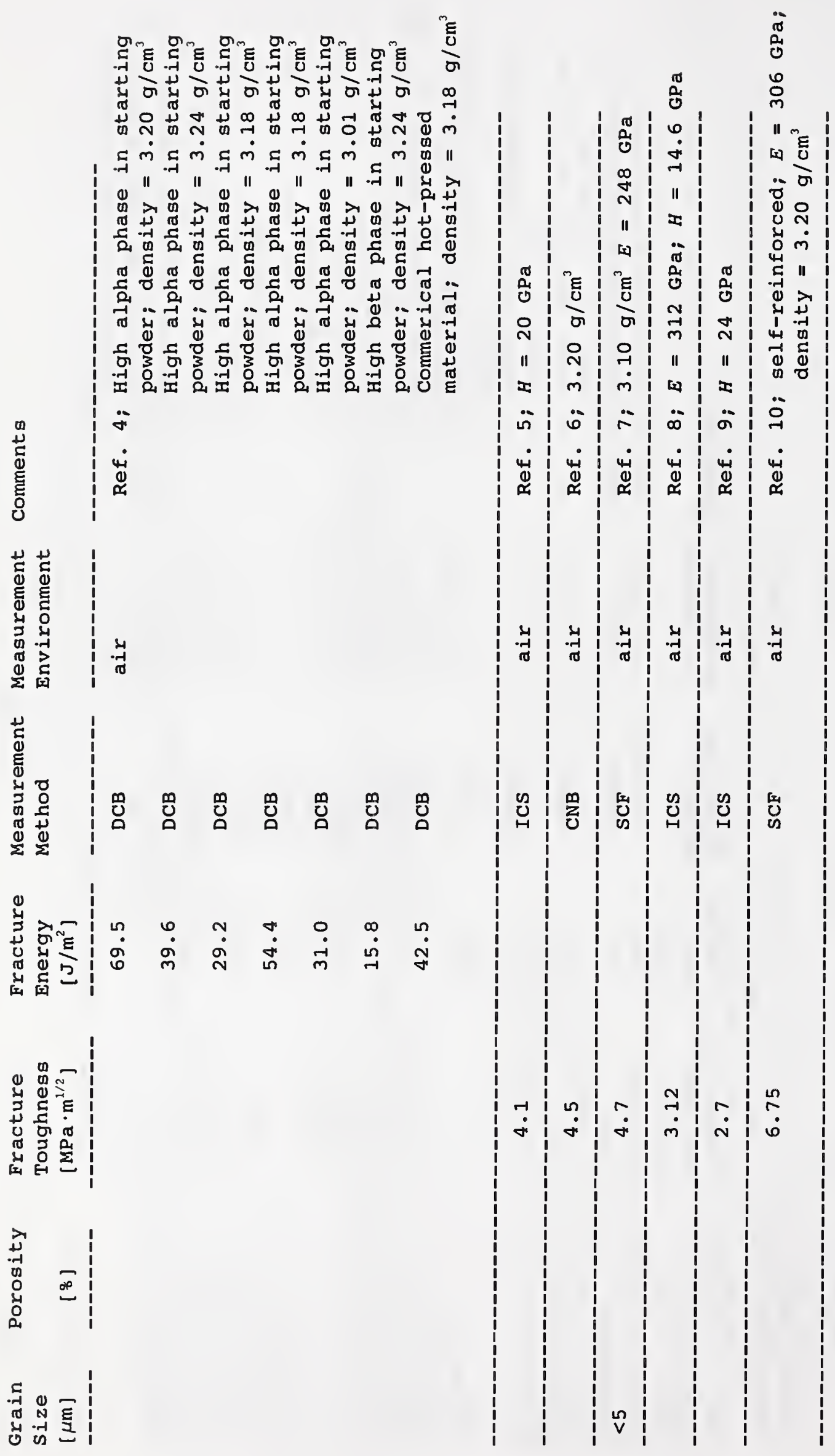



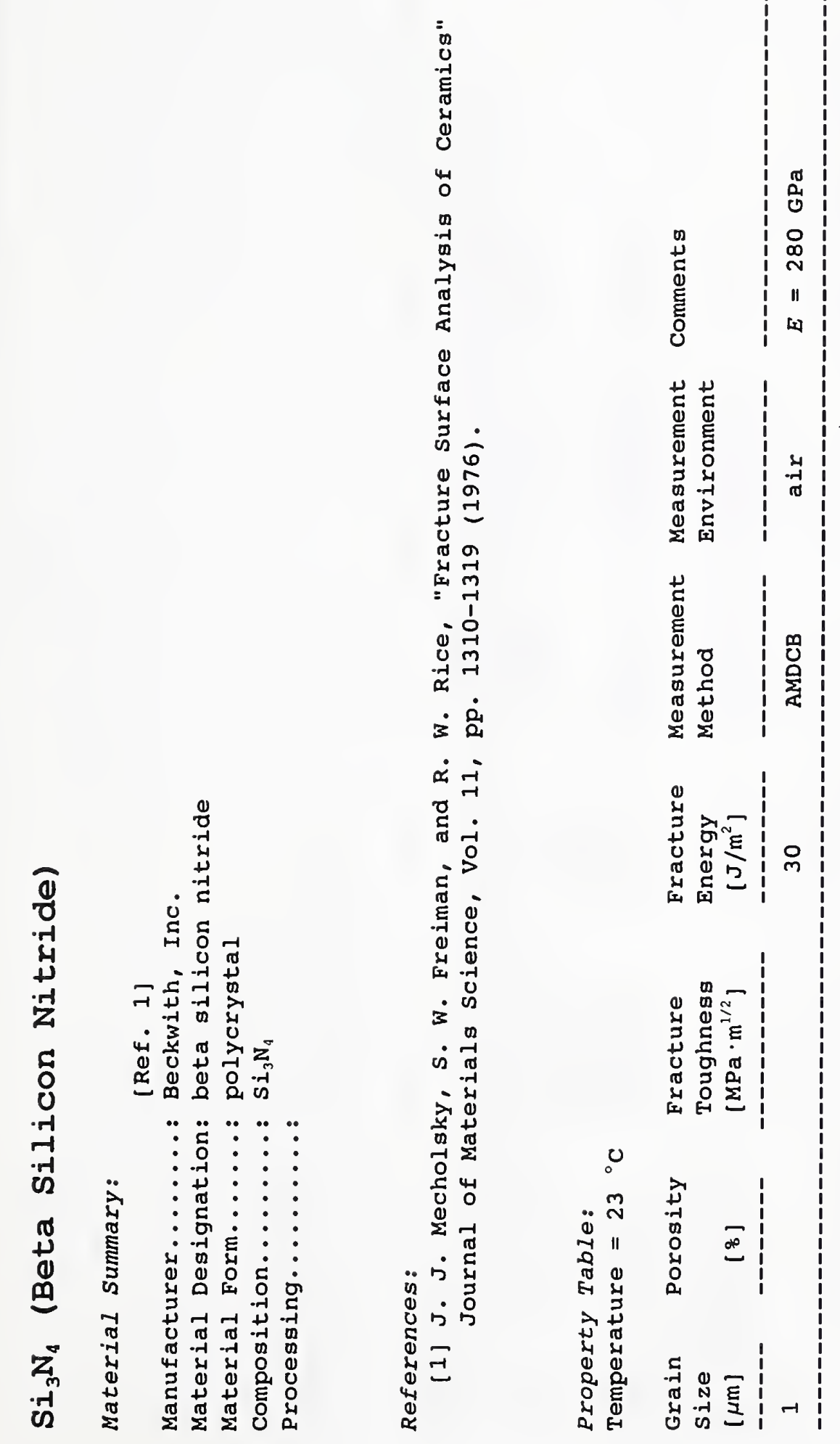


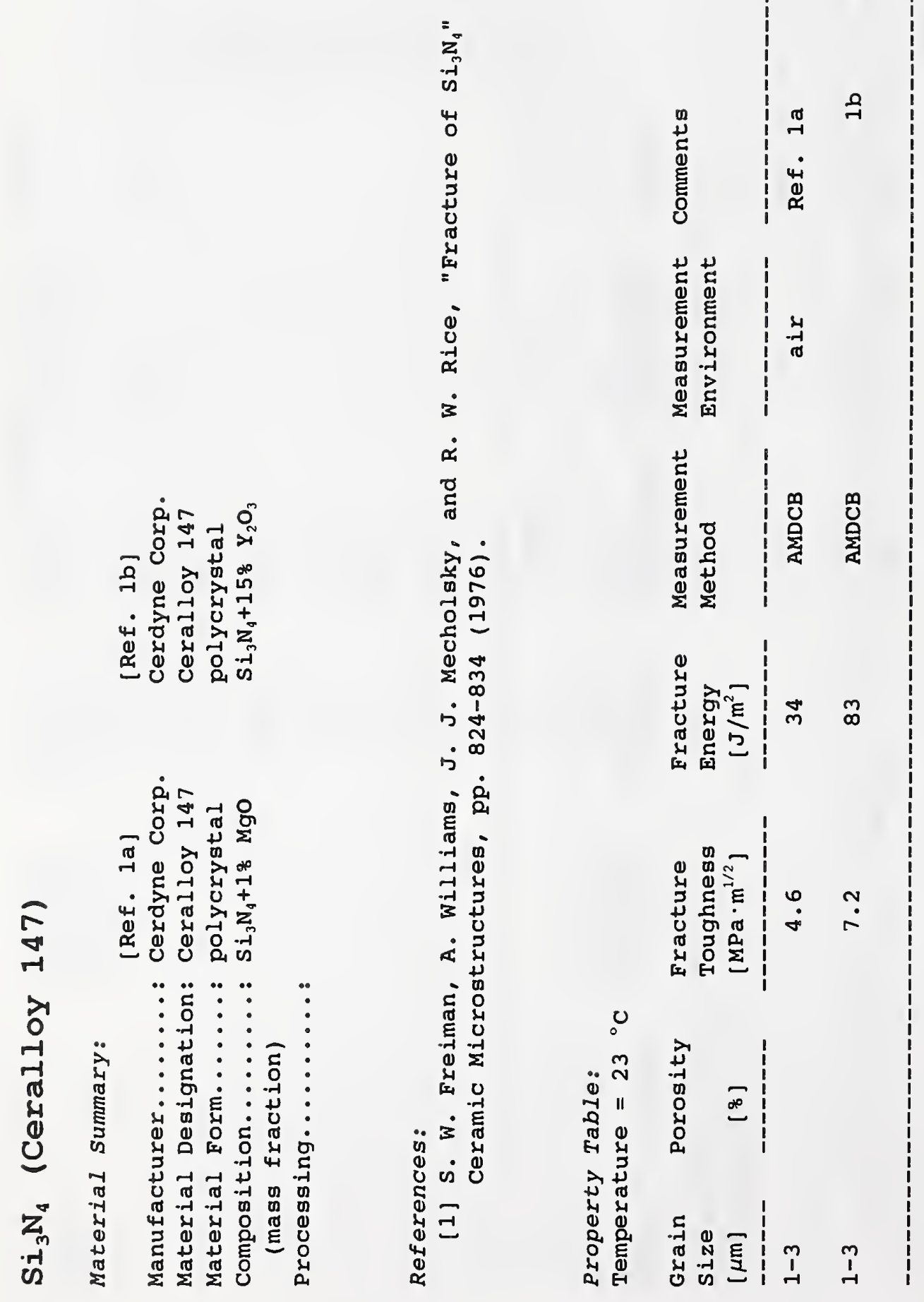

$\stackrel{1}{1}$ 


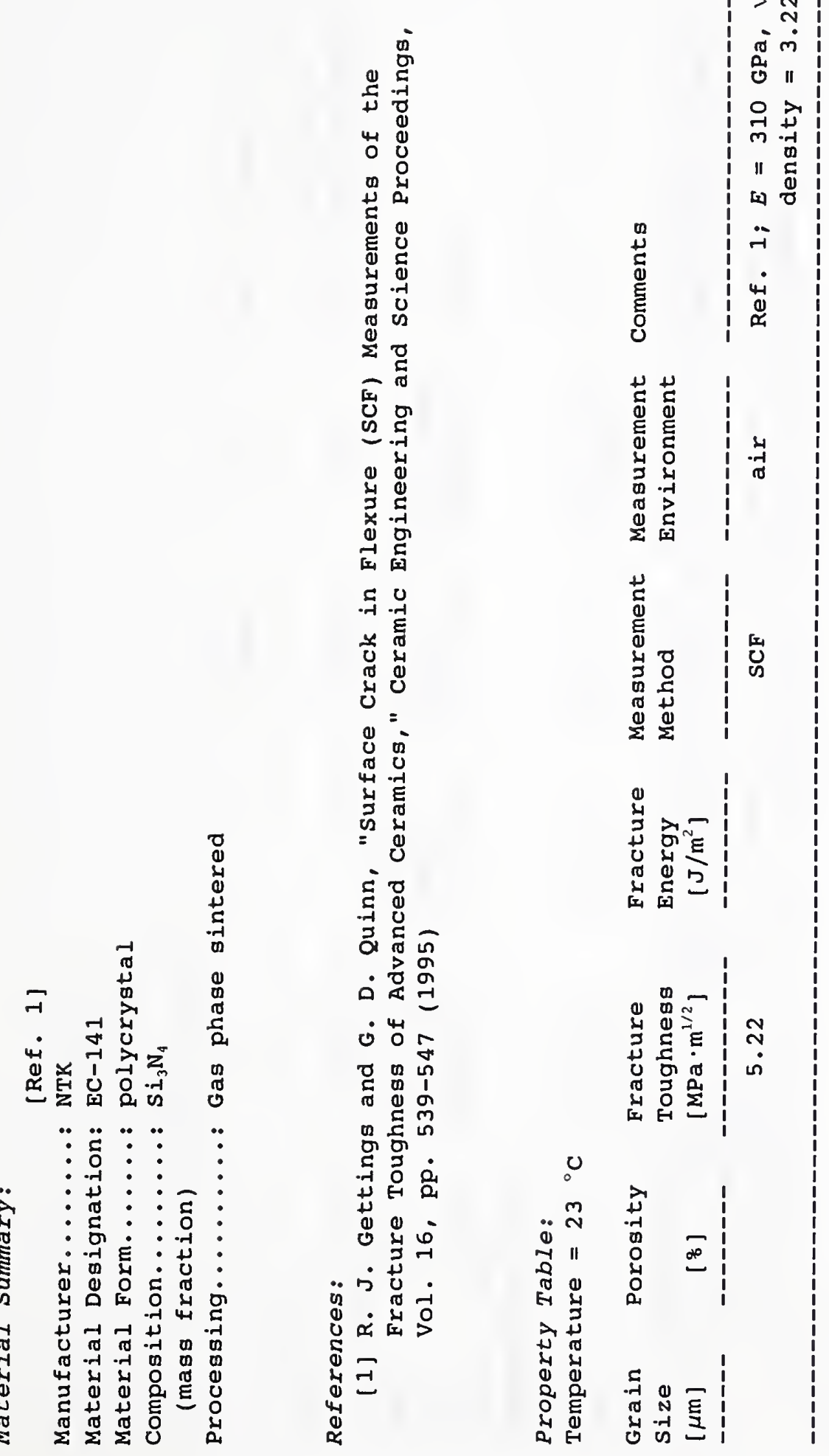




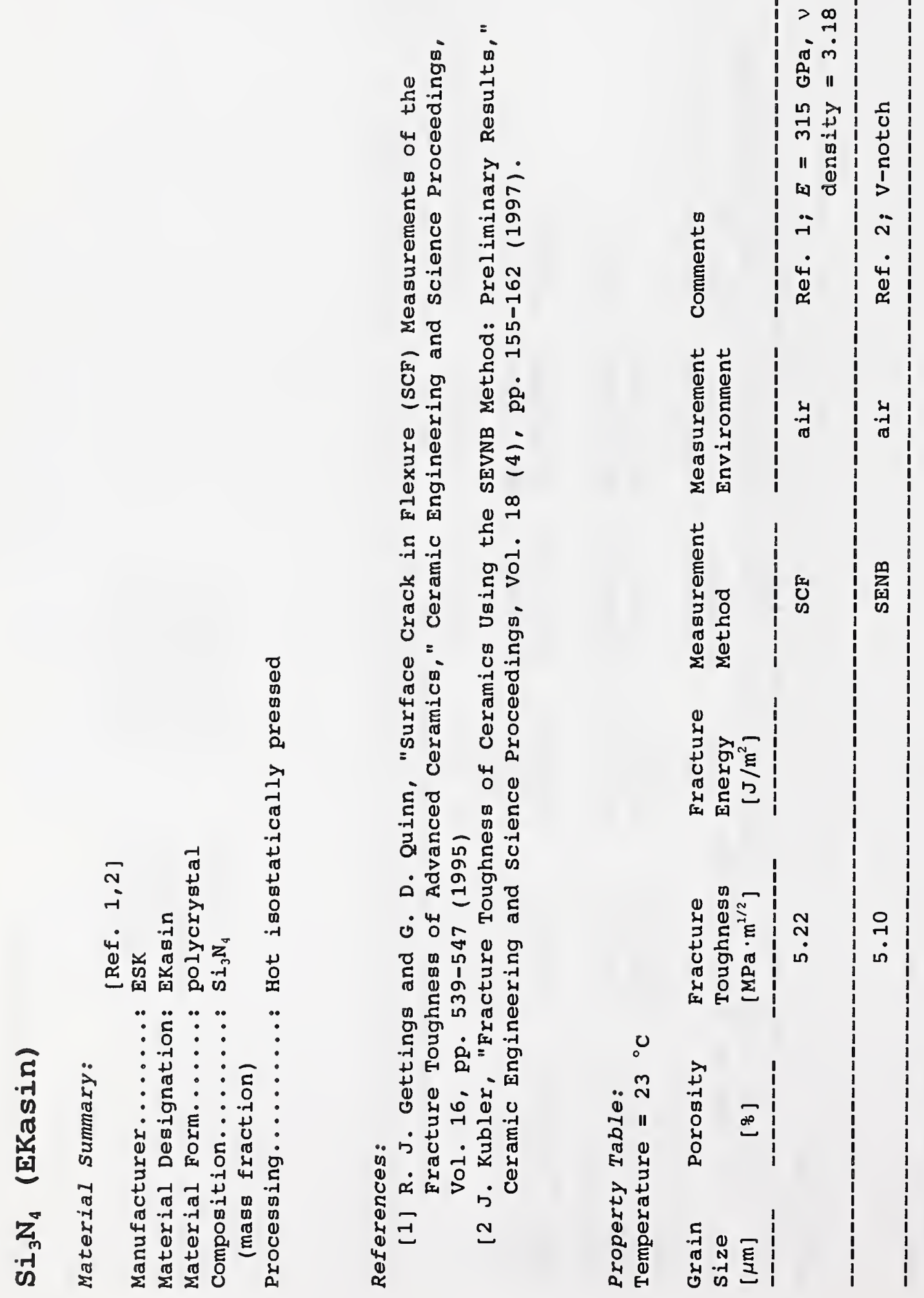



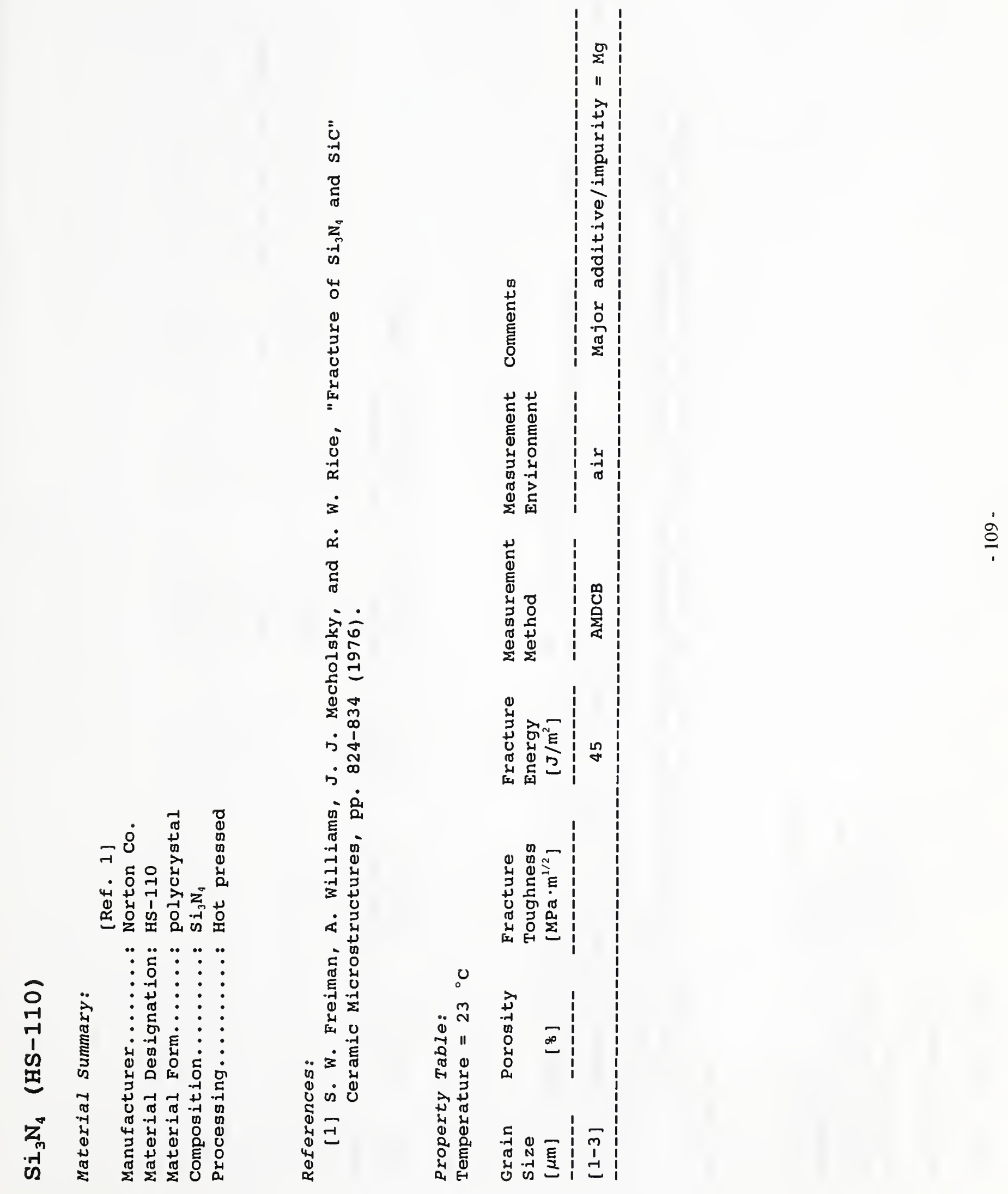

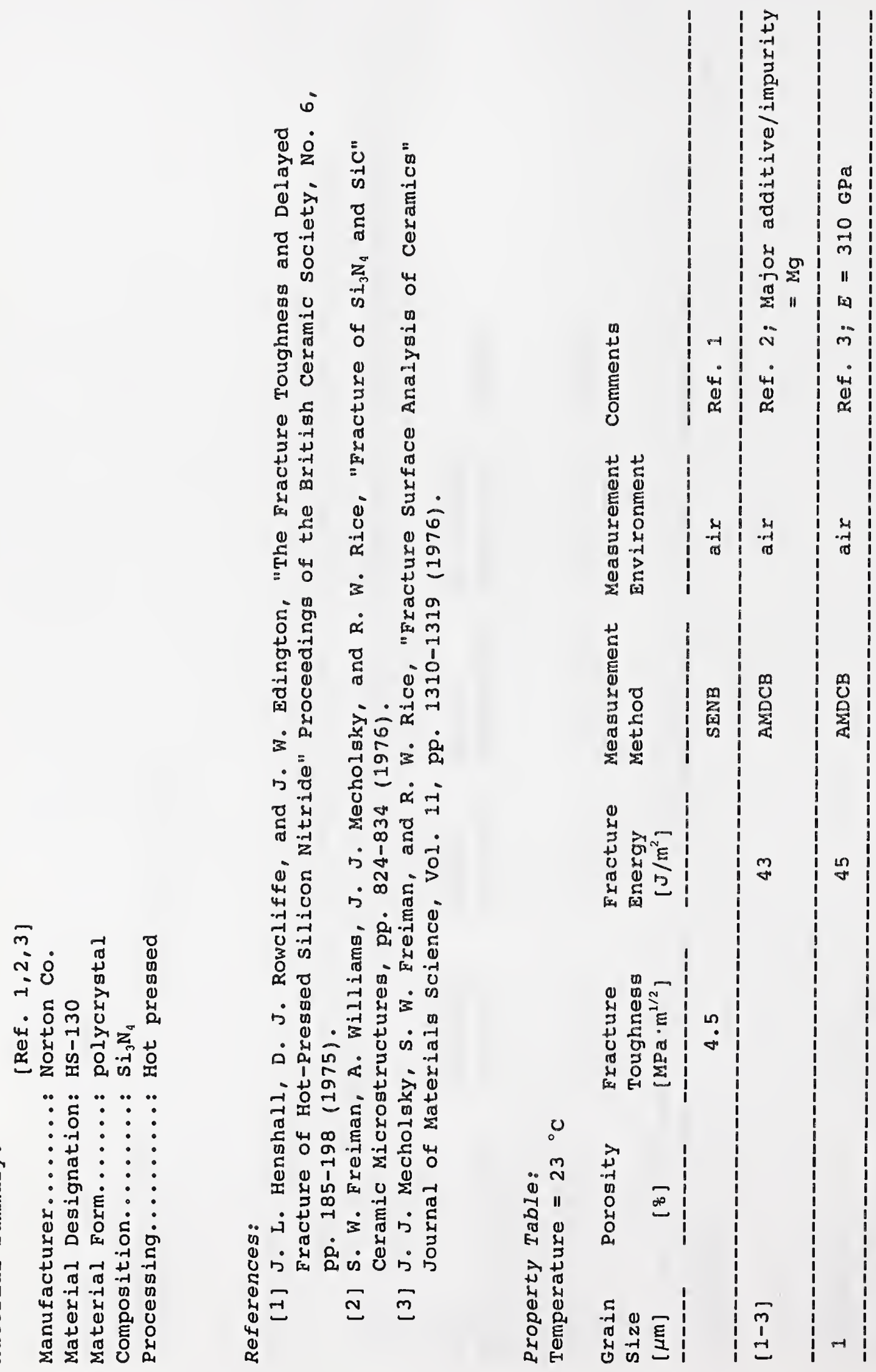


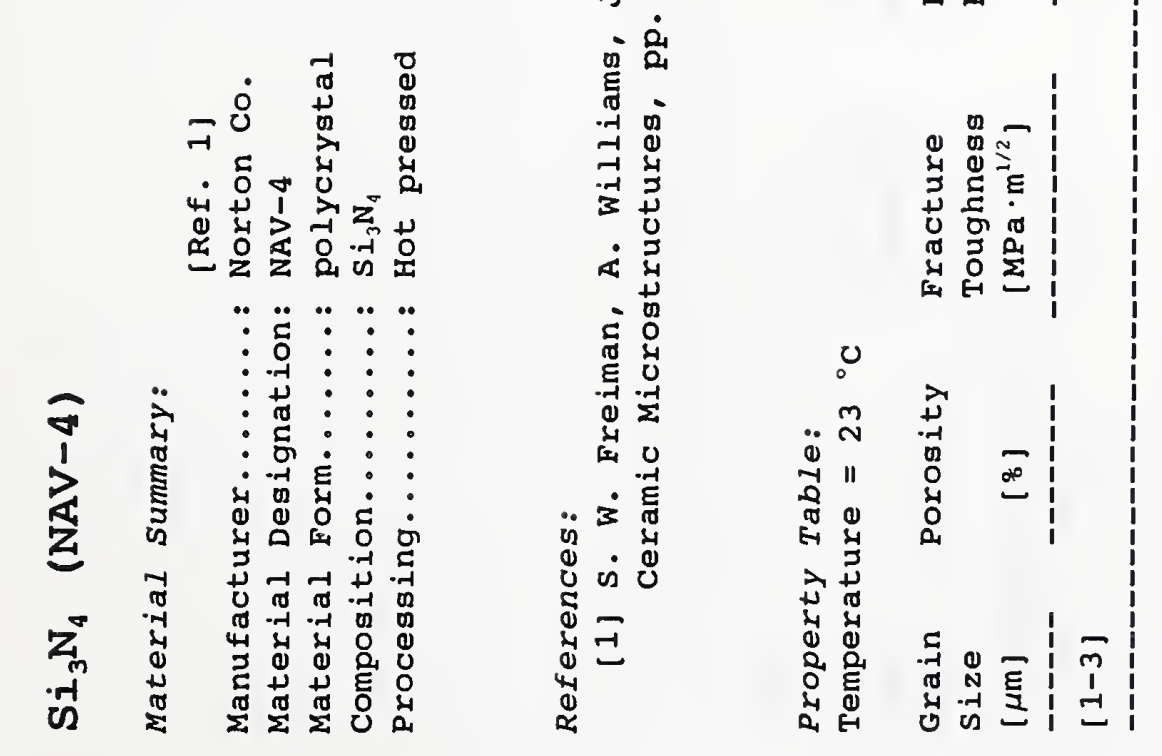




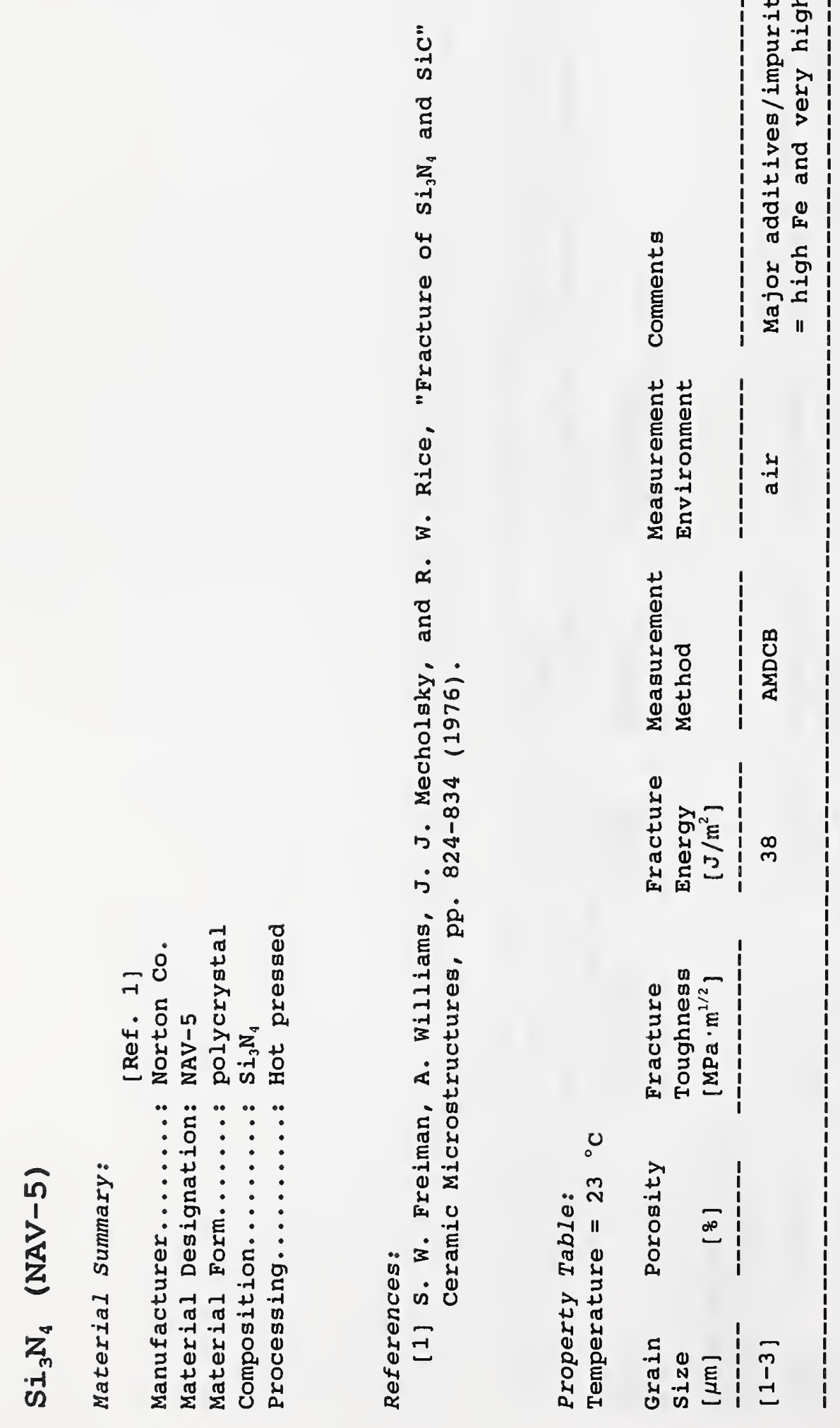



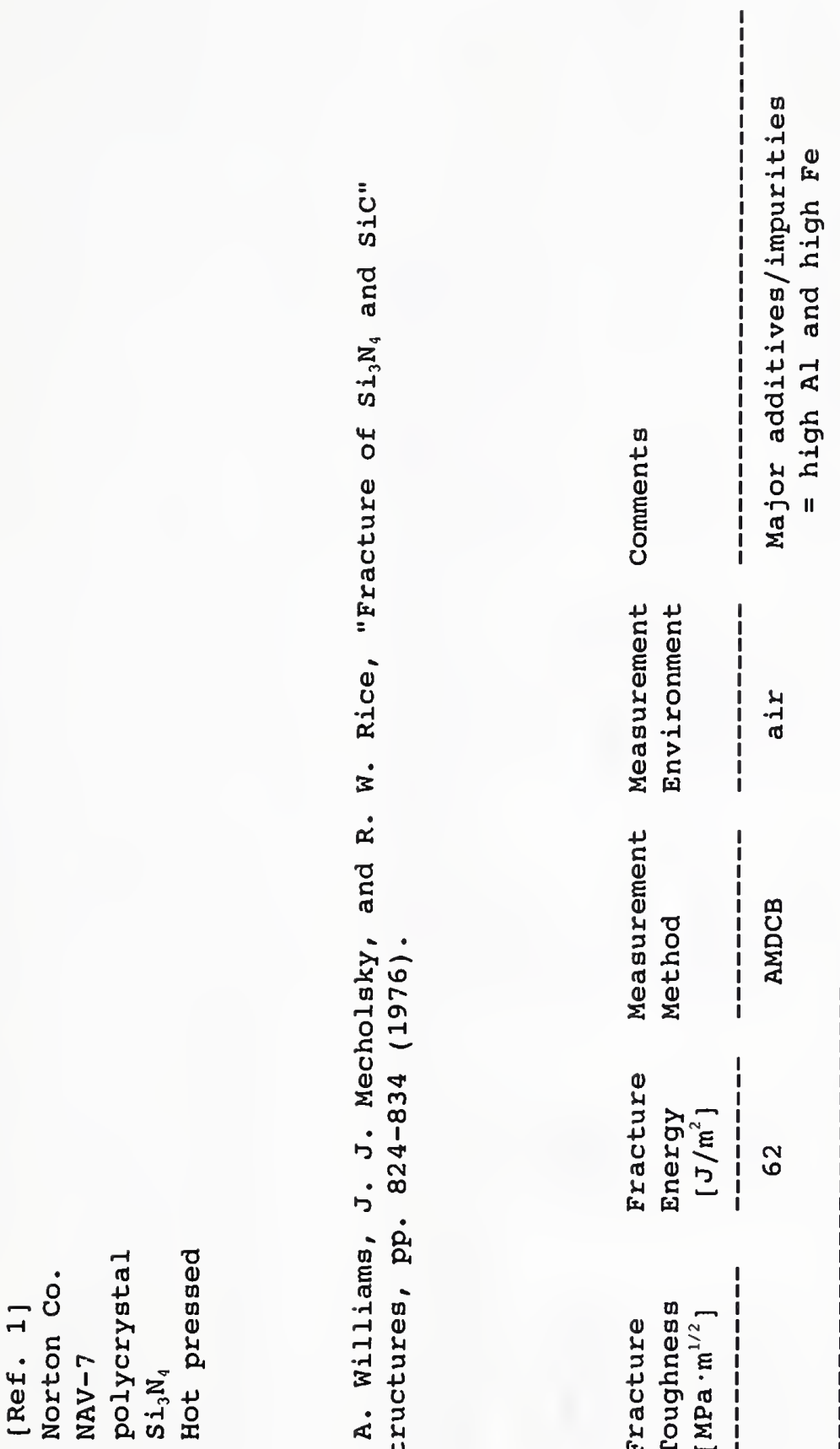

$\because \ddot{*} \cdot \ddot{*} \cdot \bullet$
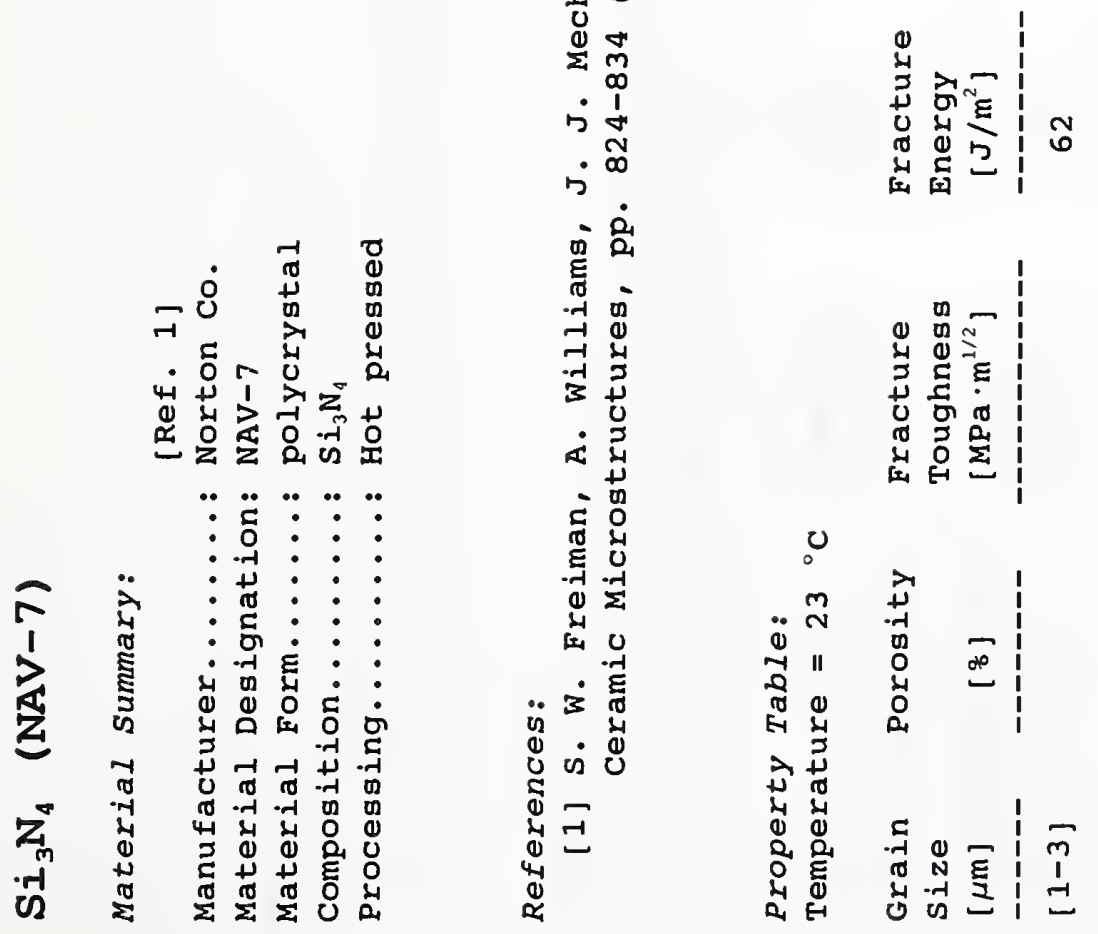


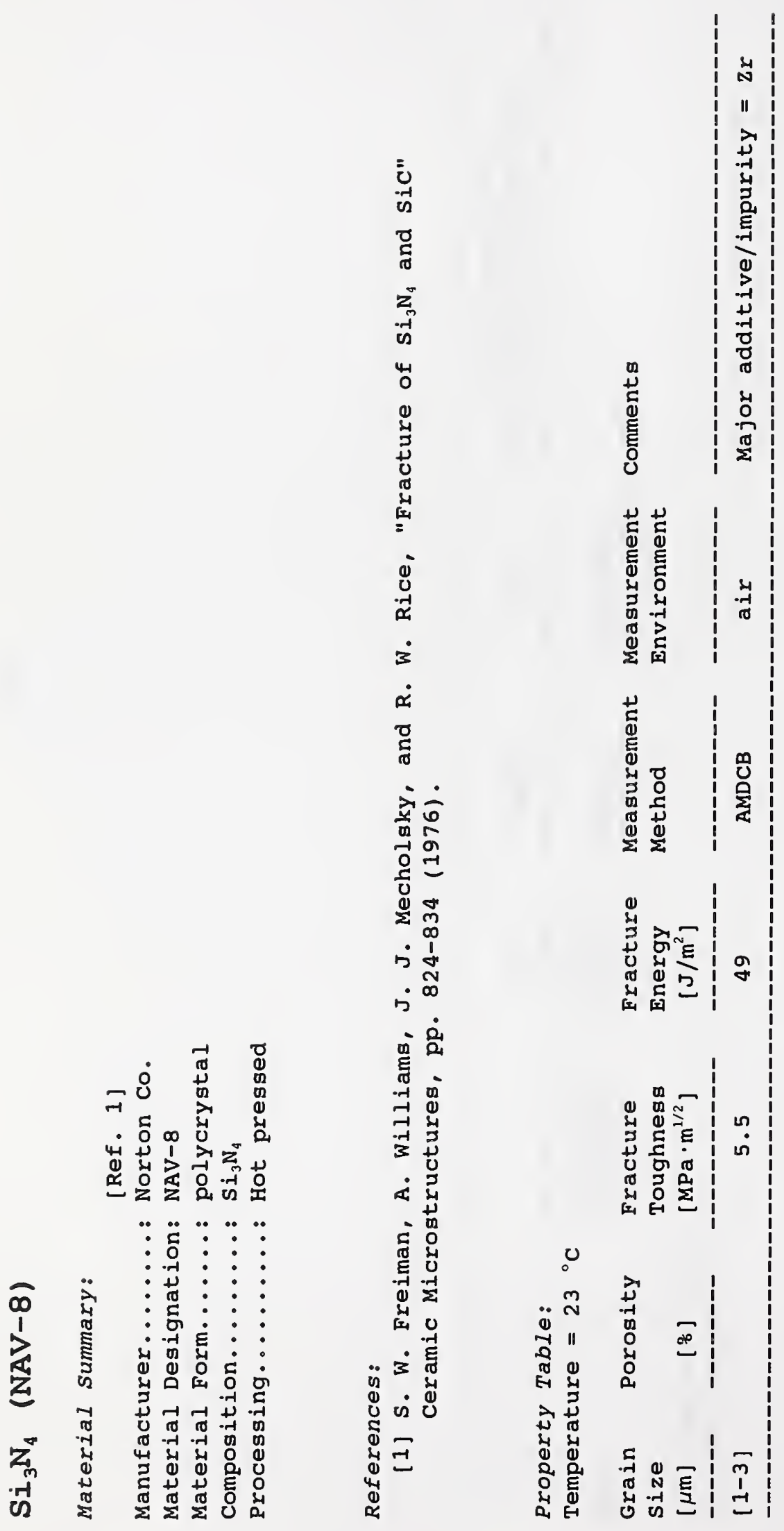




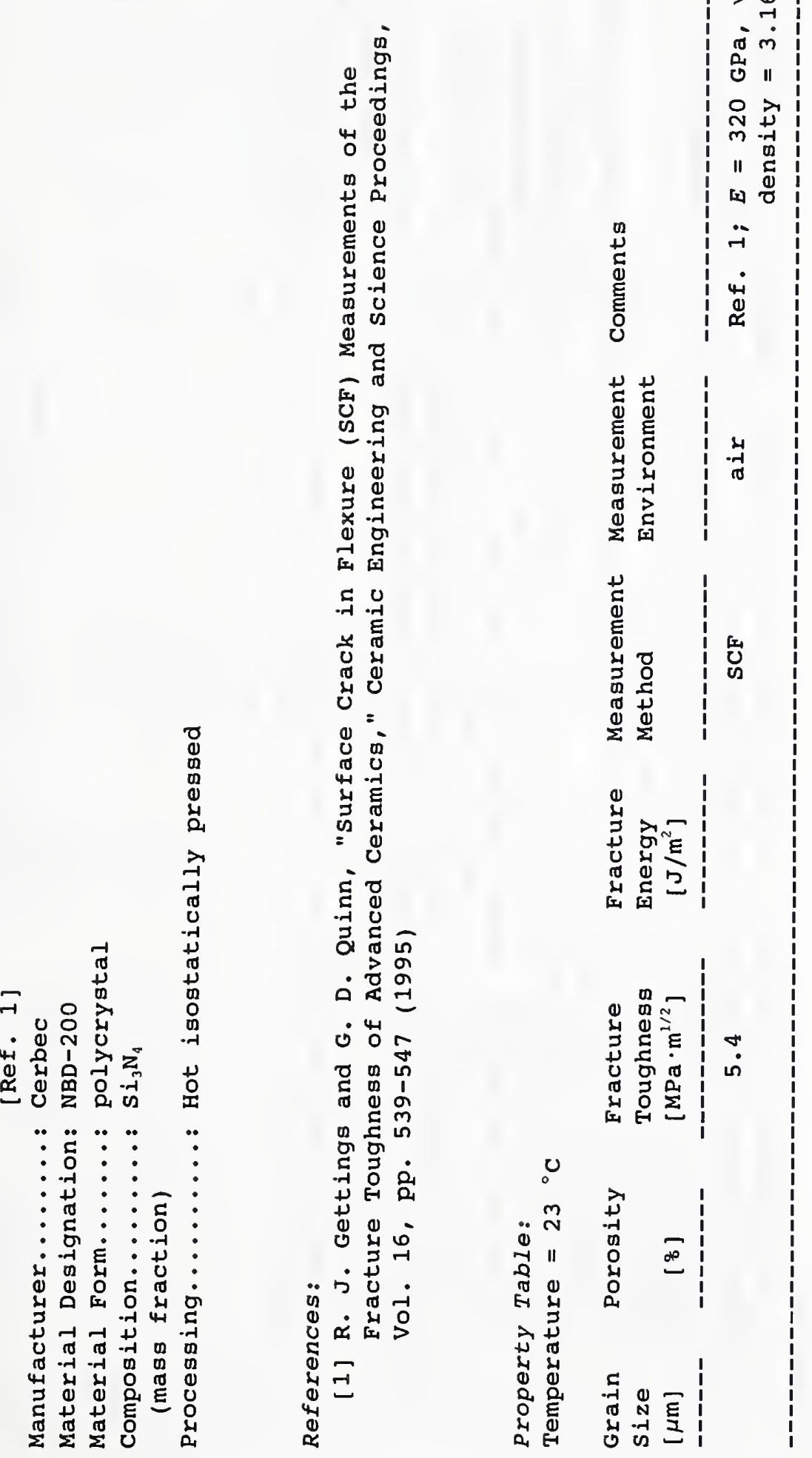



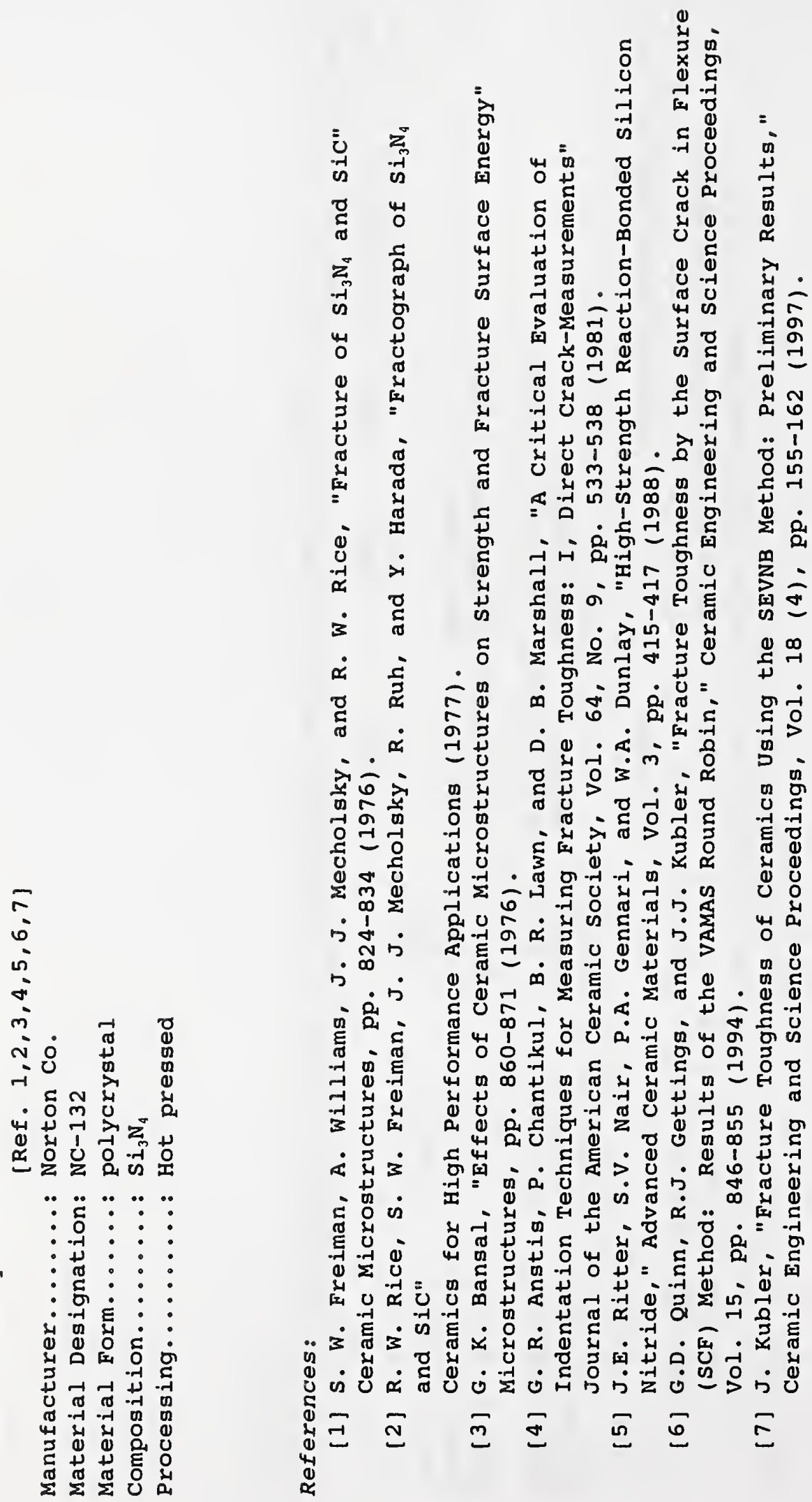


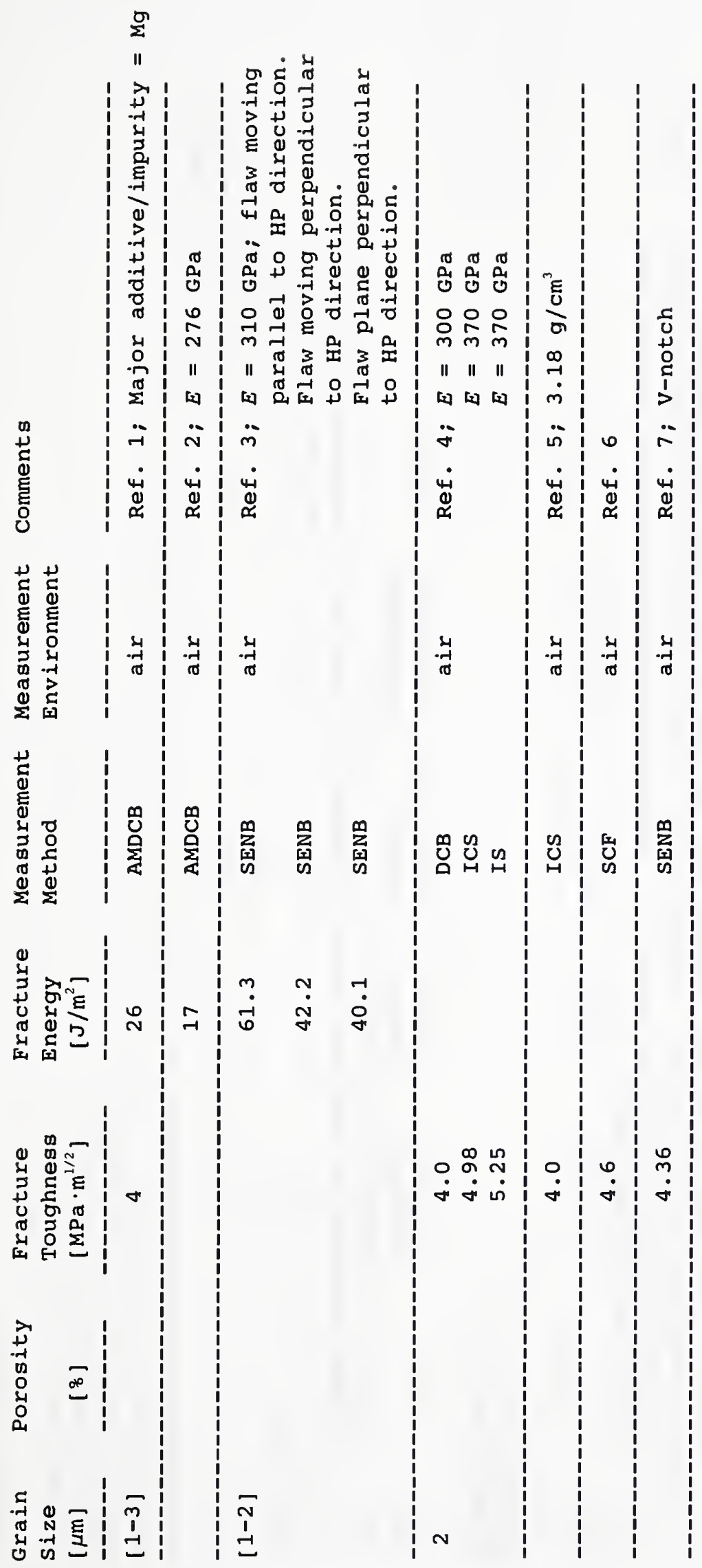



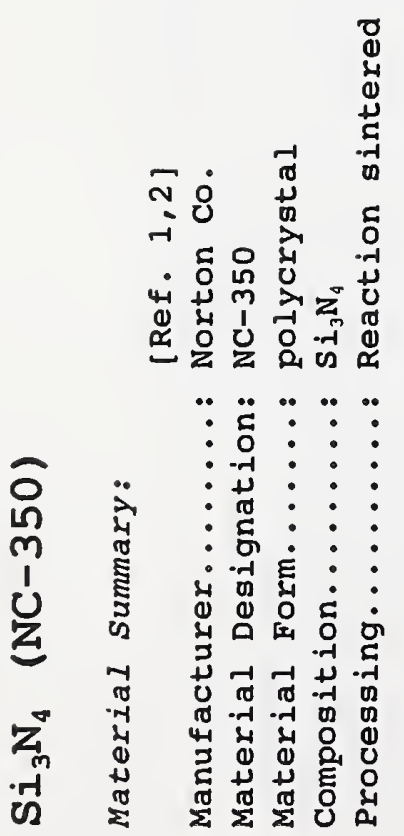

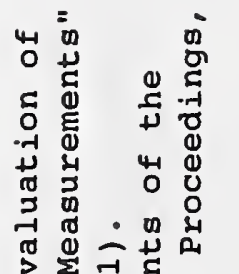

기 $\infty$ \&

$\rightarrow \frac{\pi}{U}$ ब

రુ

廿U

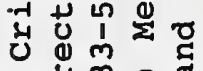

๔

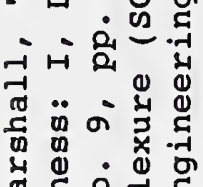

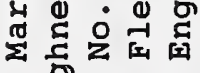

бо म

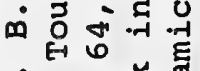

口 0.04

ช

त 0

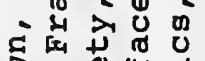

o o.

号宅讨

.न्म

$\therefore$ 岁

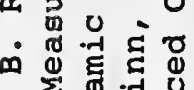

¡ म

节然 ठ व

ป

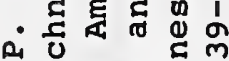

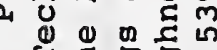

- E 完

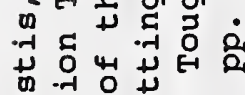

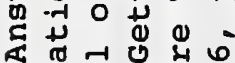

选元

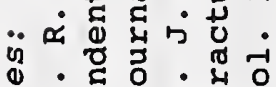

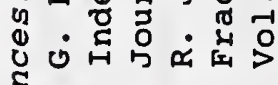

离

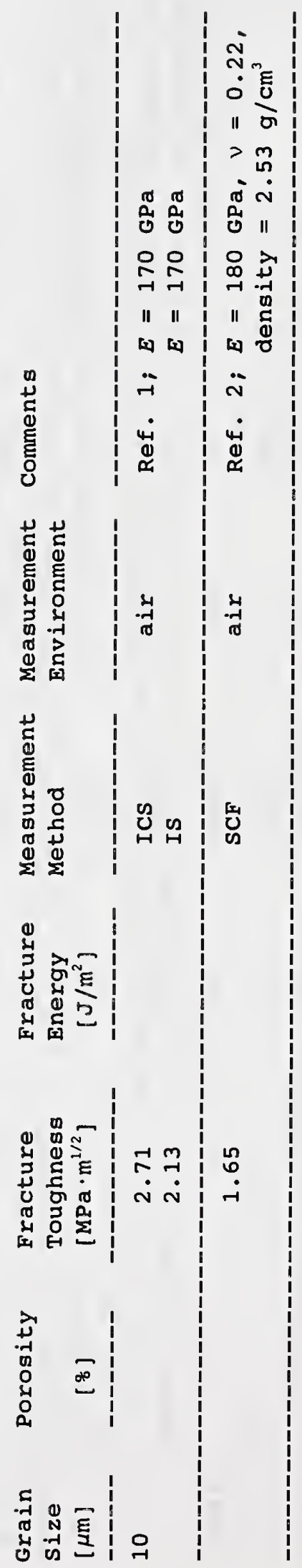



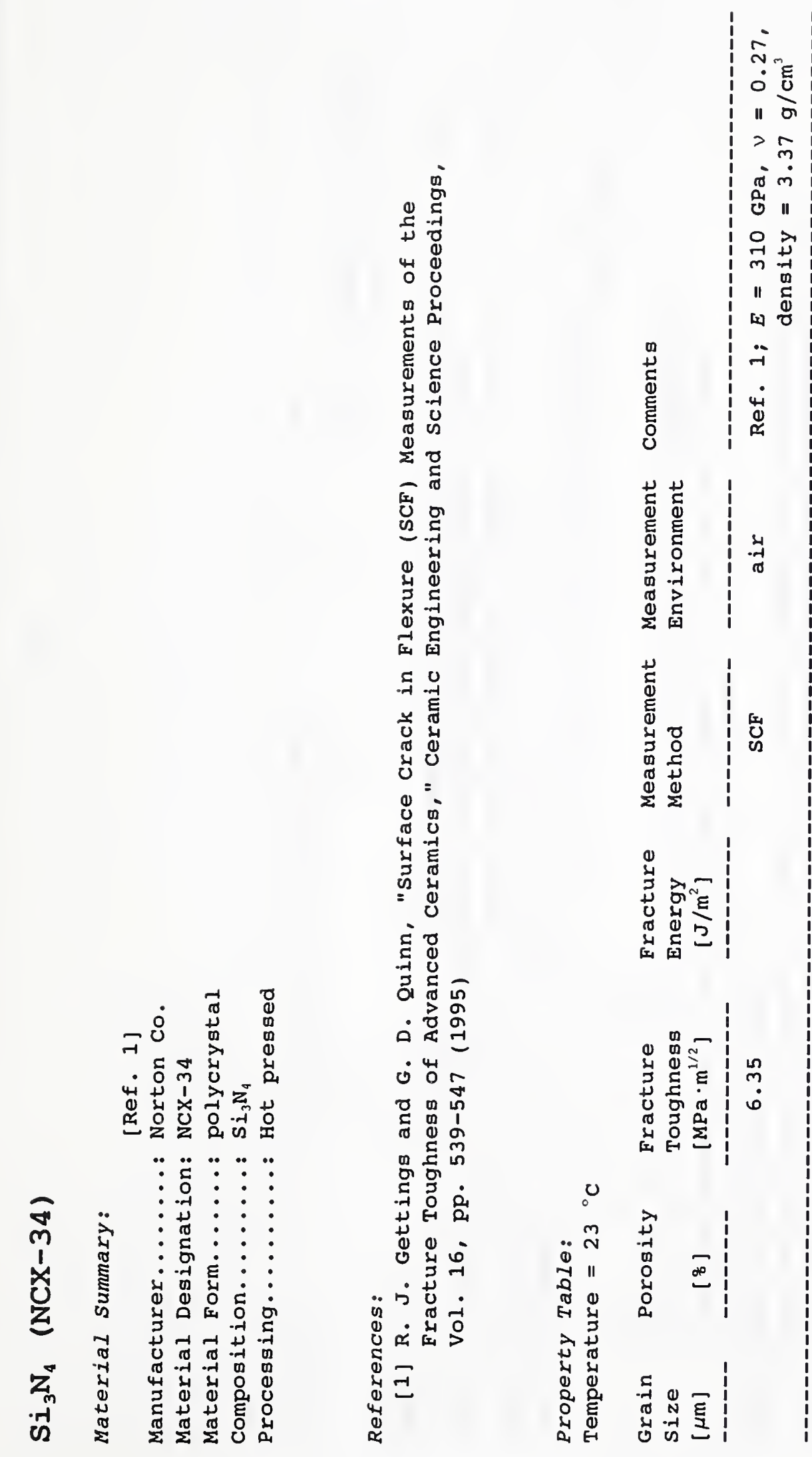

.

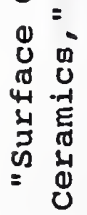

हृ

ว
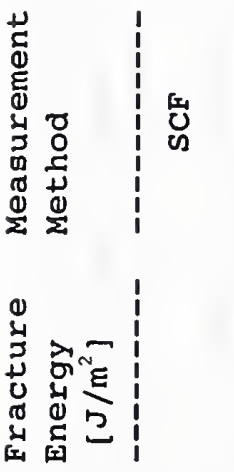


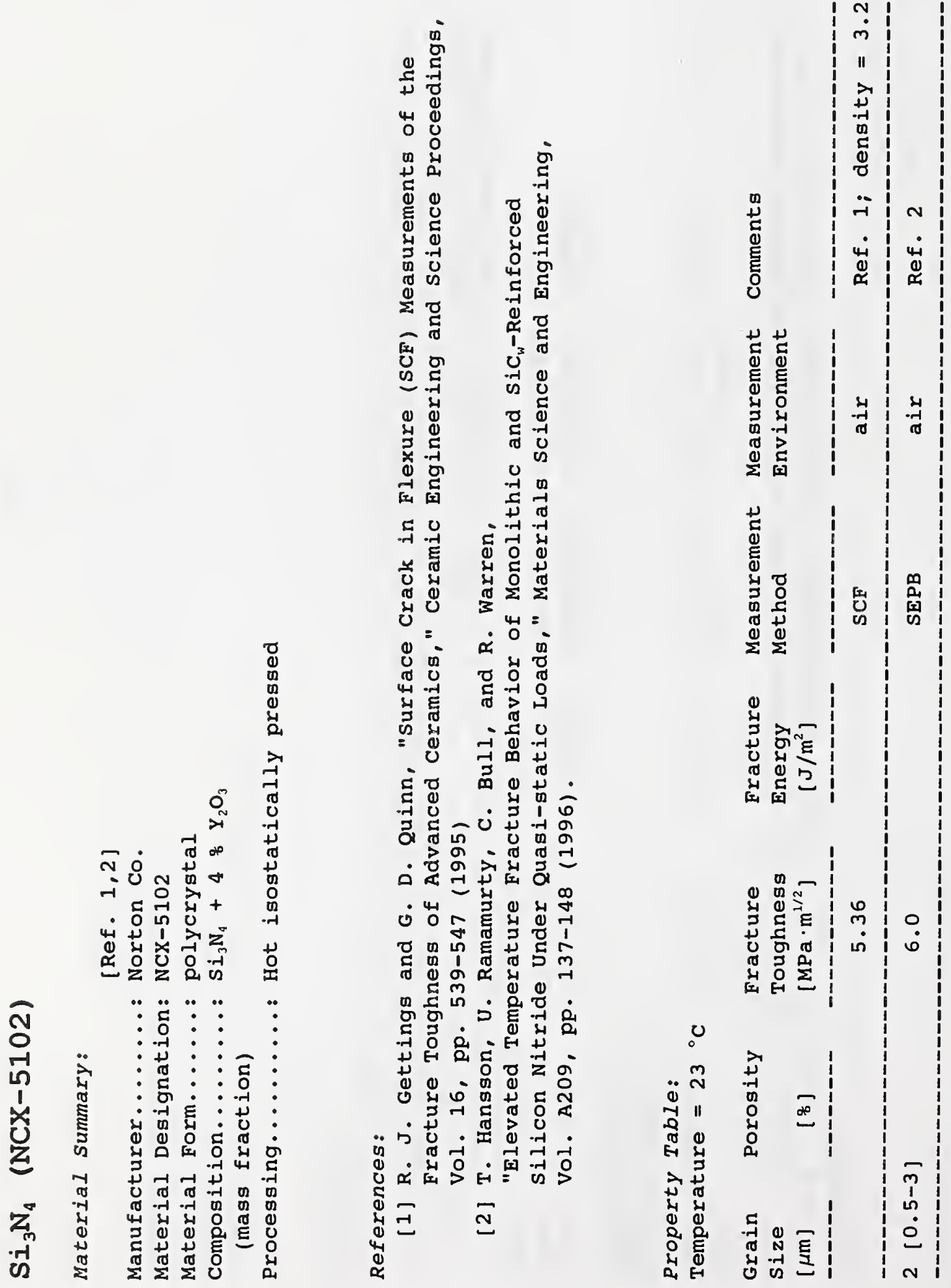




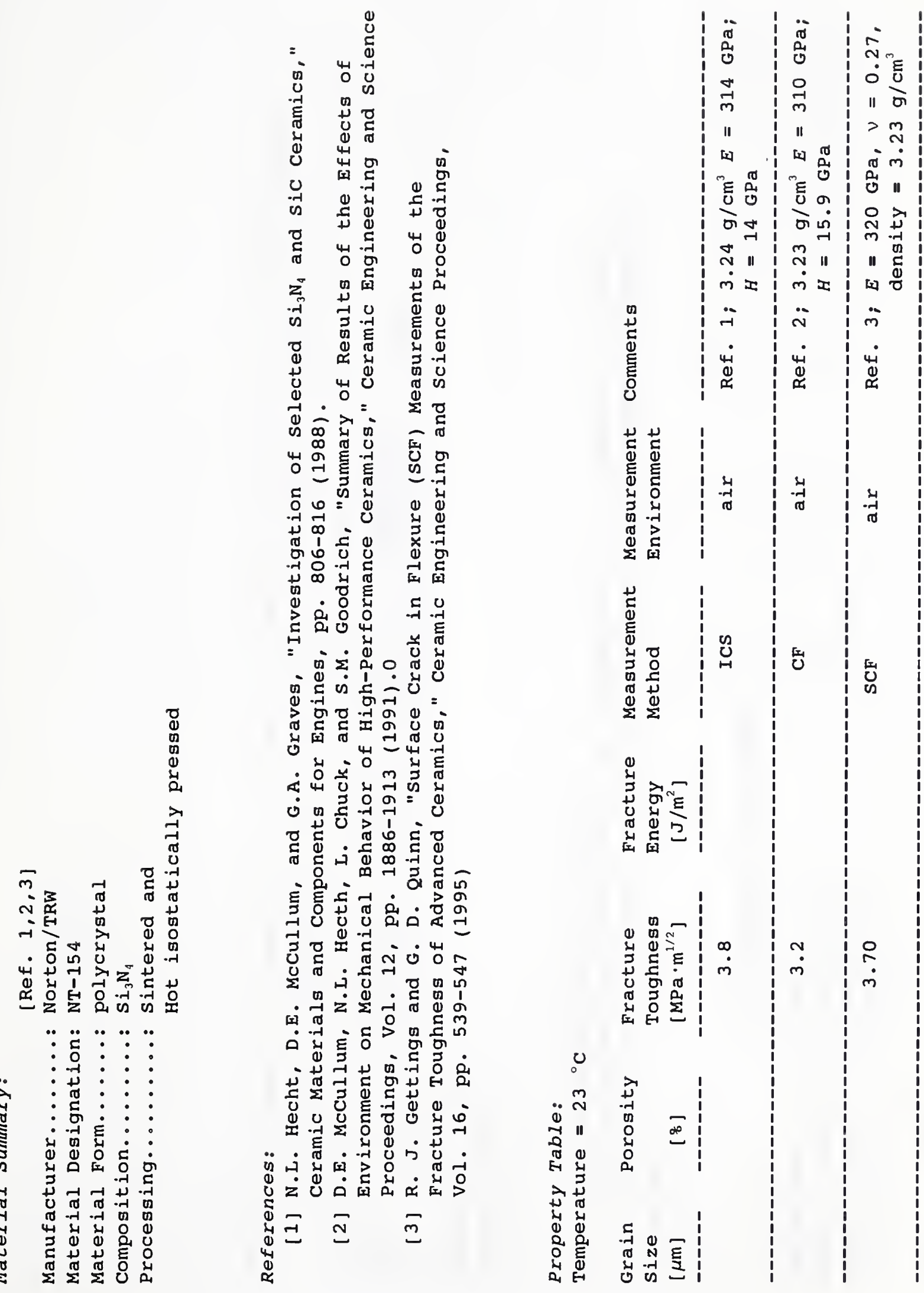



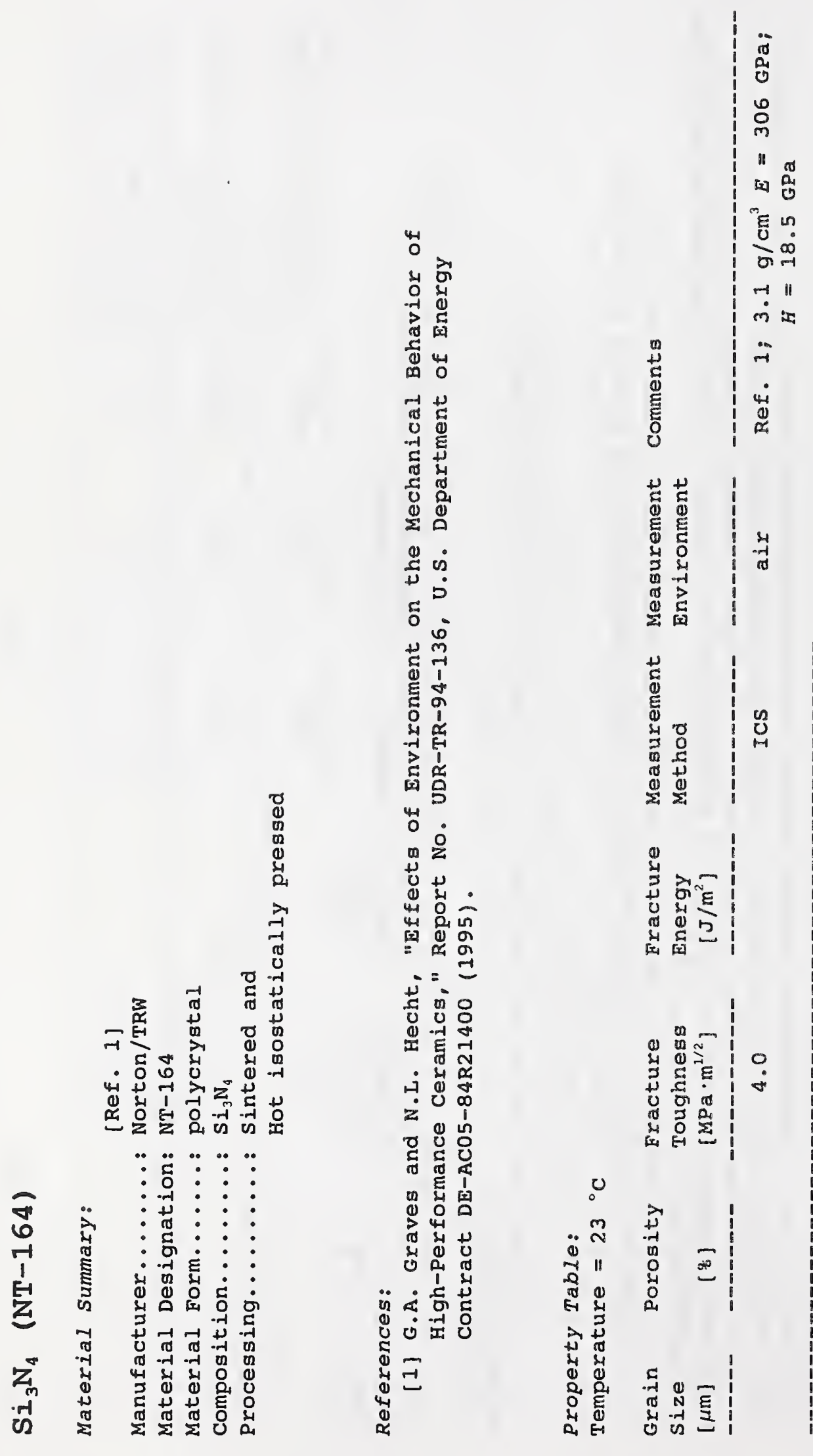

$\frac{1}{1}$ 


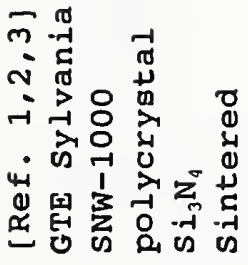

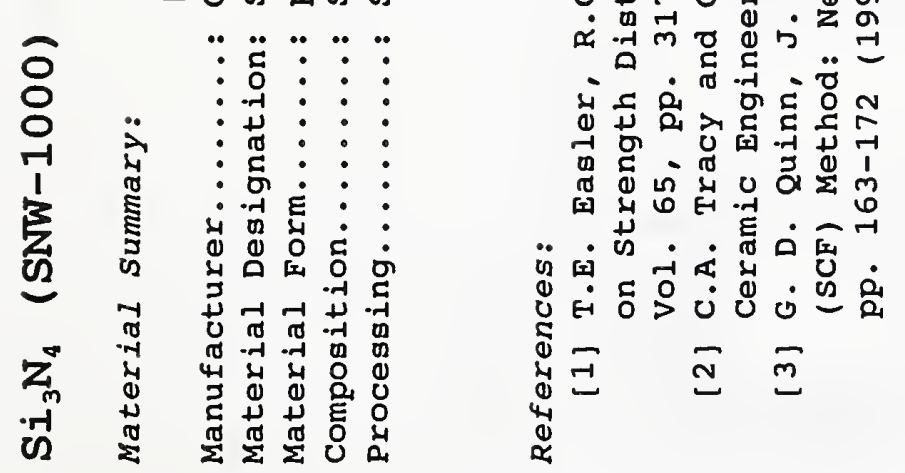

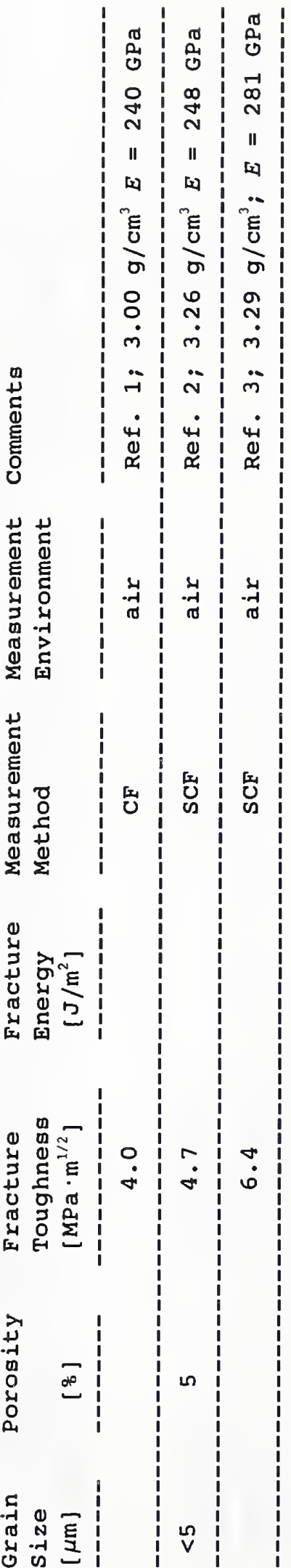




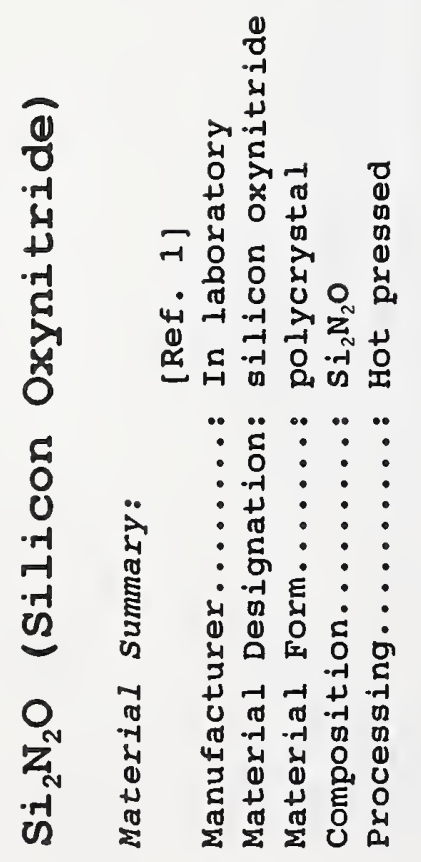

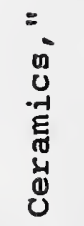

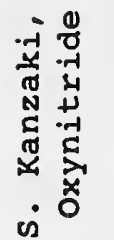

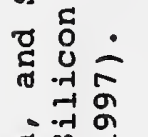

कै के

跑

겅

员思京

ty

之这宫

- O

क्ष

击

势

$x$ 류

is $\frac{\pi}{0}$

选造

है

z

- 00

ن

- 出

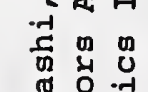

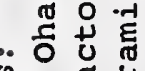

iे 0 盛

造焉

पै
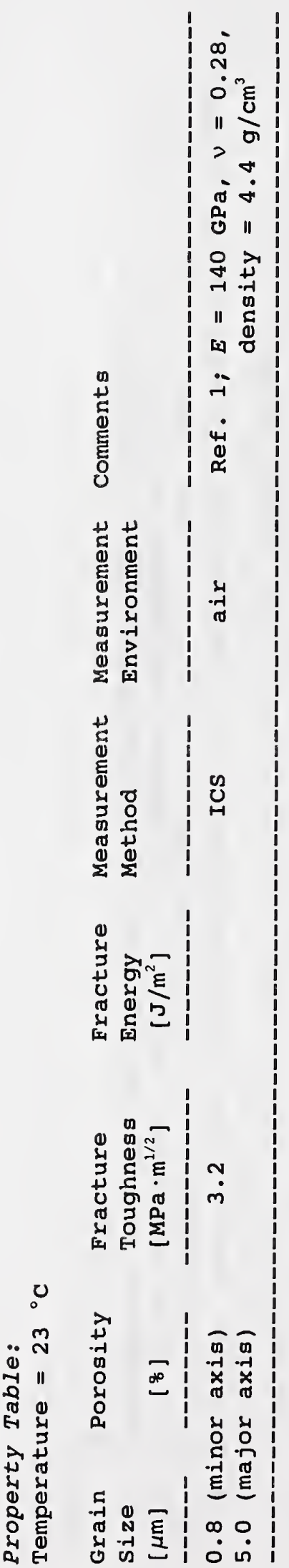


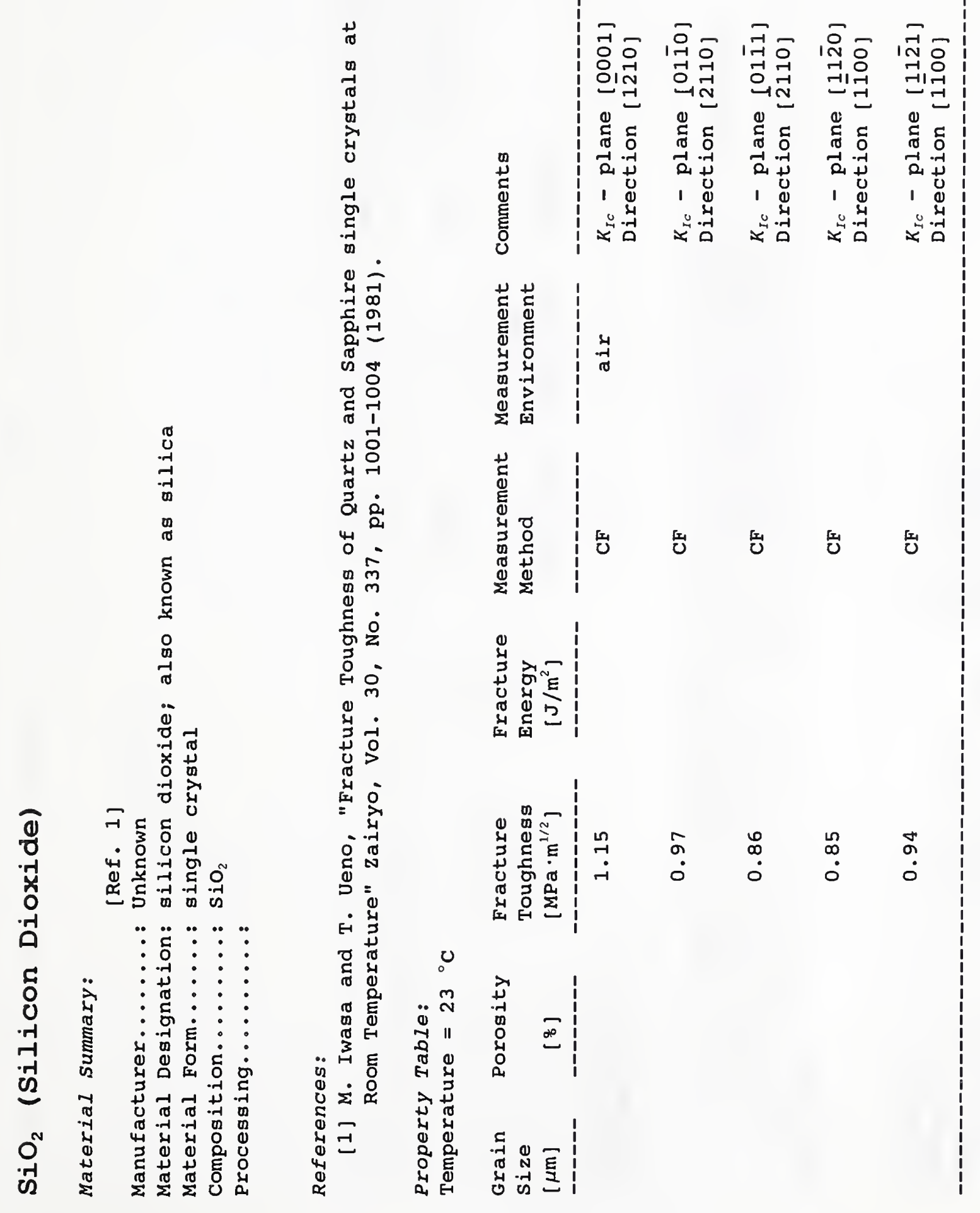

$\stackrel{9}{9}$ 

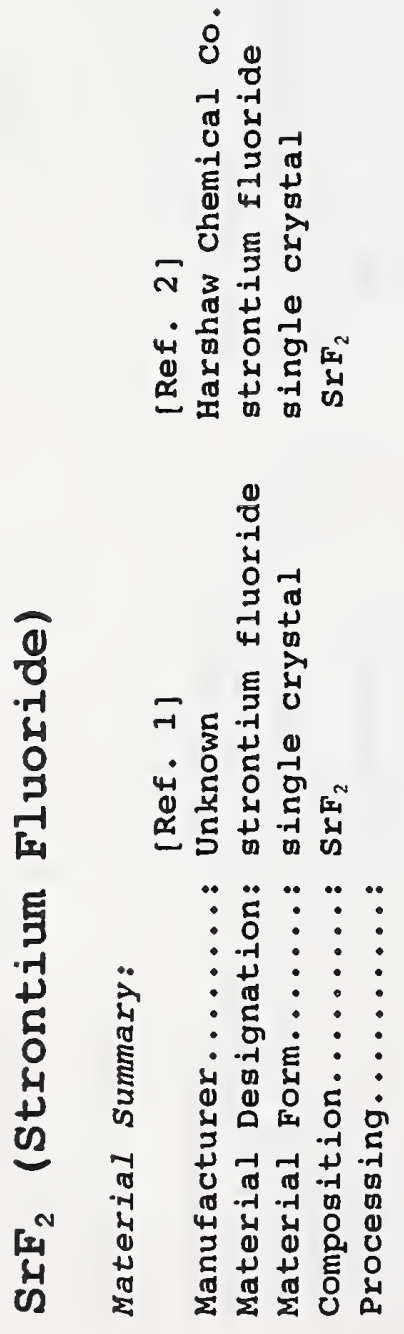

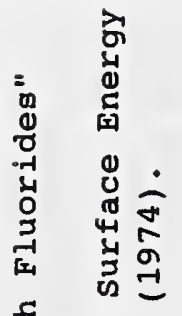

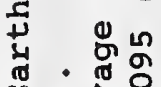

$\rightarrow$

1.

द्न

要

素尔这

$m$ o

न बून

중

ธำ

\%

\%

的的枈

Q

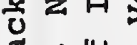

近合㟧

类

वृ

年。

o

o- $\begin{gathered}-1 \\ \text { o- }\end{gathered}$

- خ 㫕

\%

व

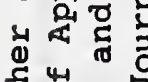

可岁 $\mathrm{N}$ 号

兽苟=

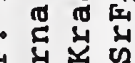

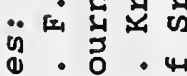

过号告㟧

峁

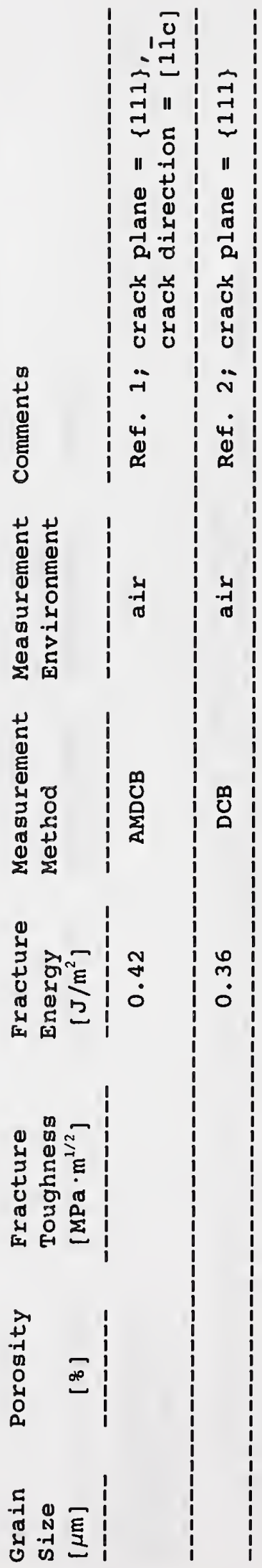

守 


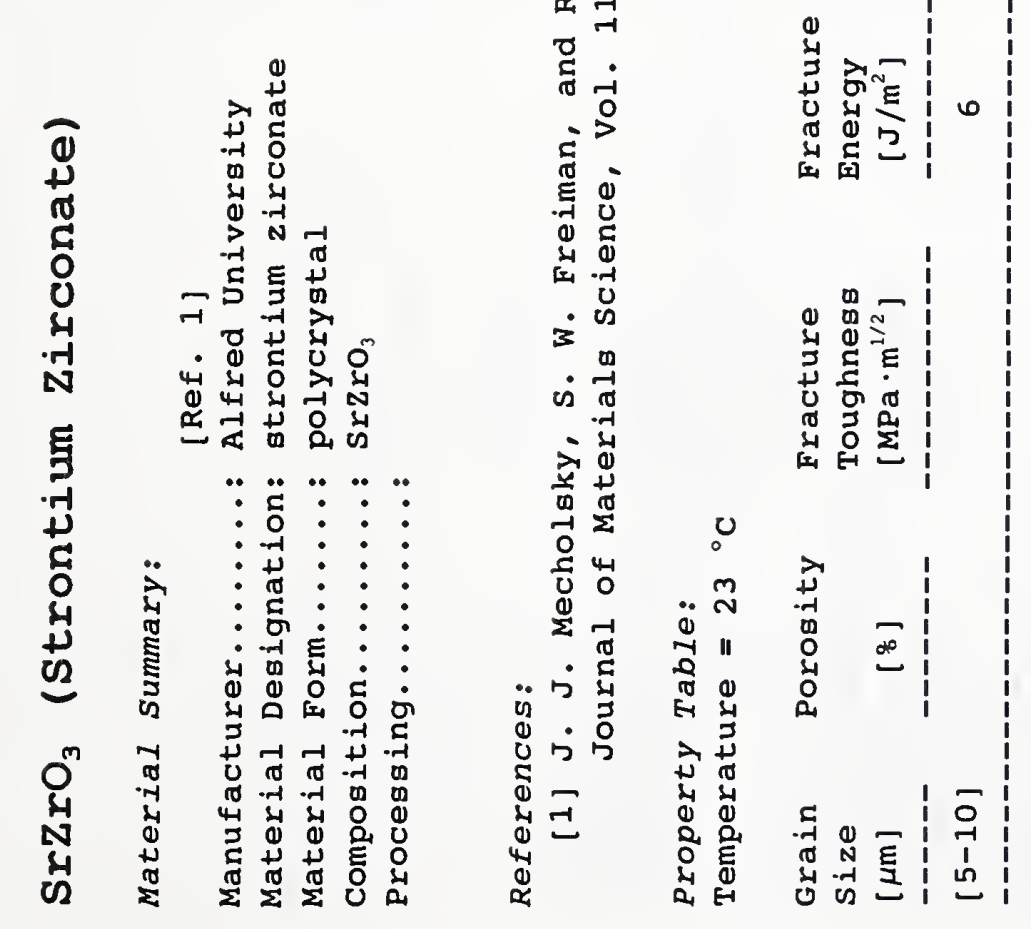




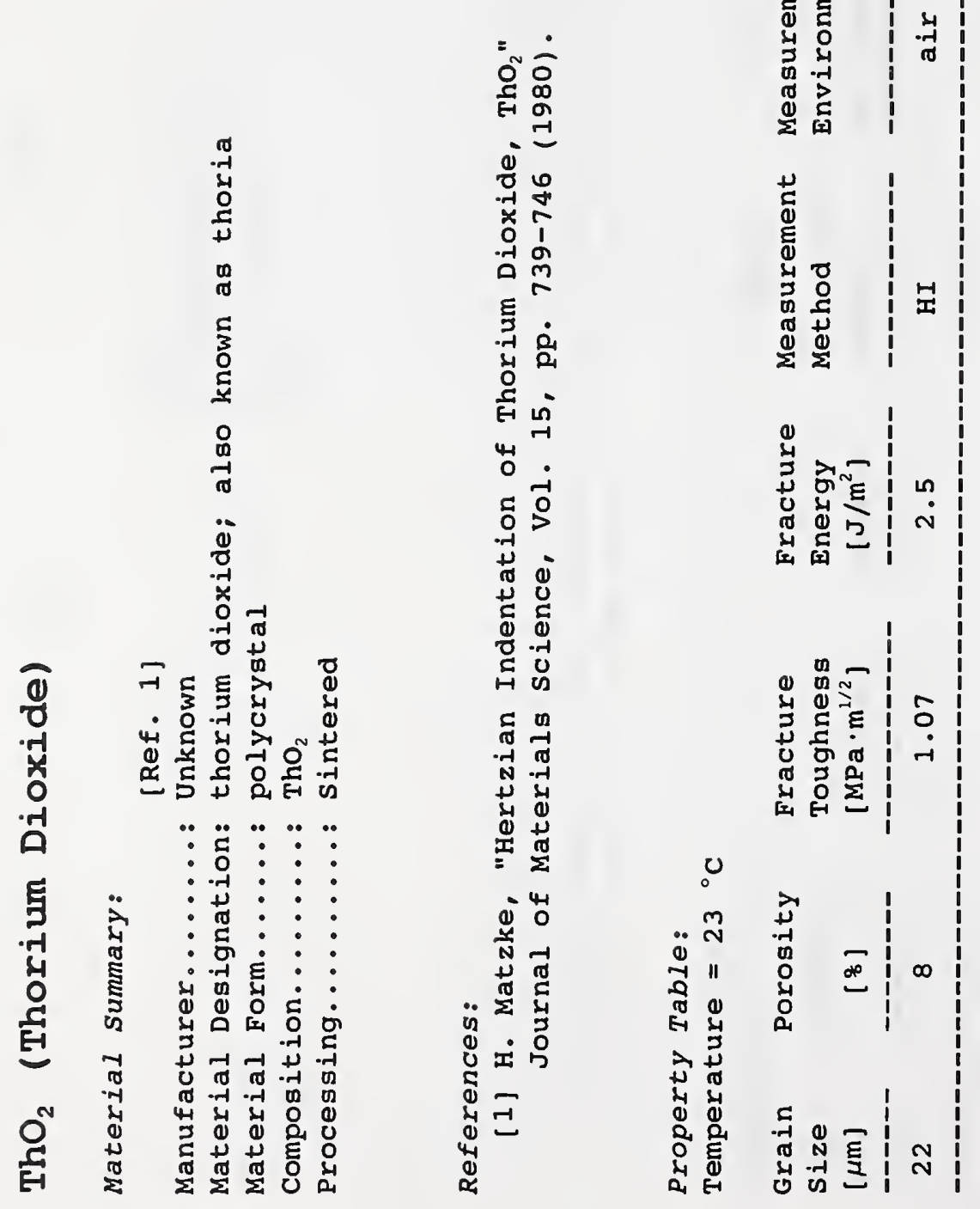

$\stackrel{\infty}{\stackrel{\infty}{u}}$ 


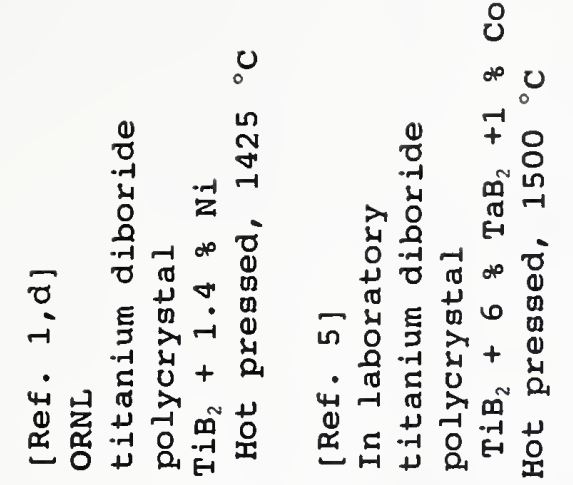
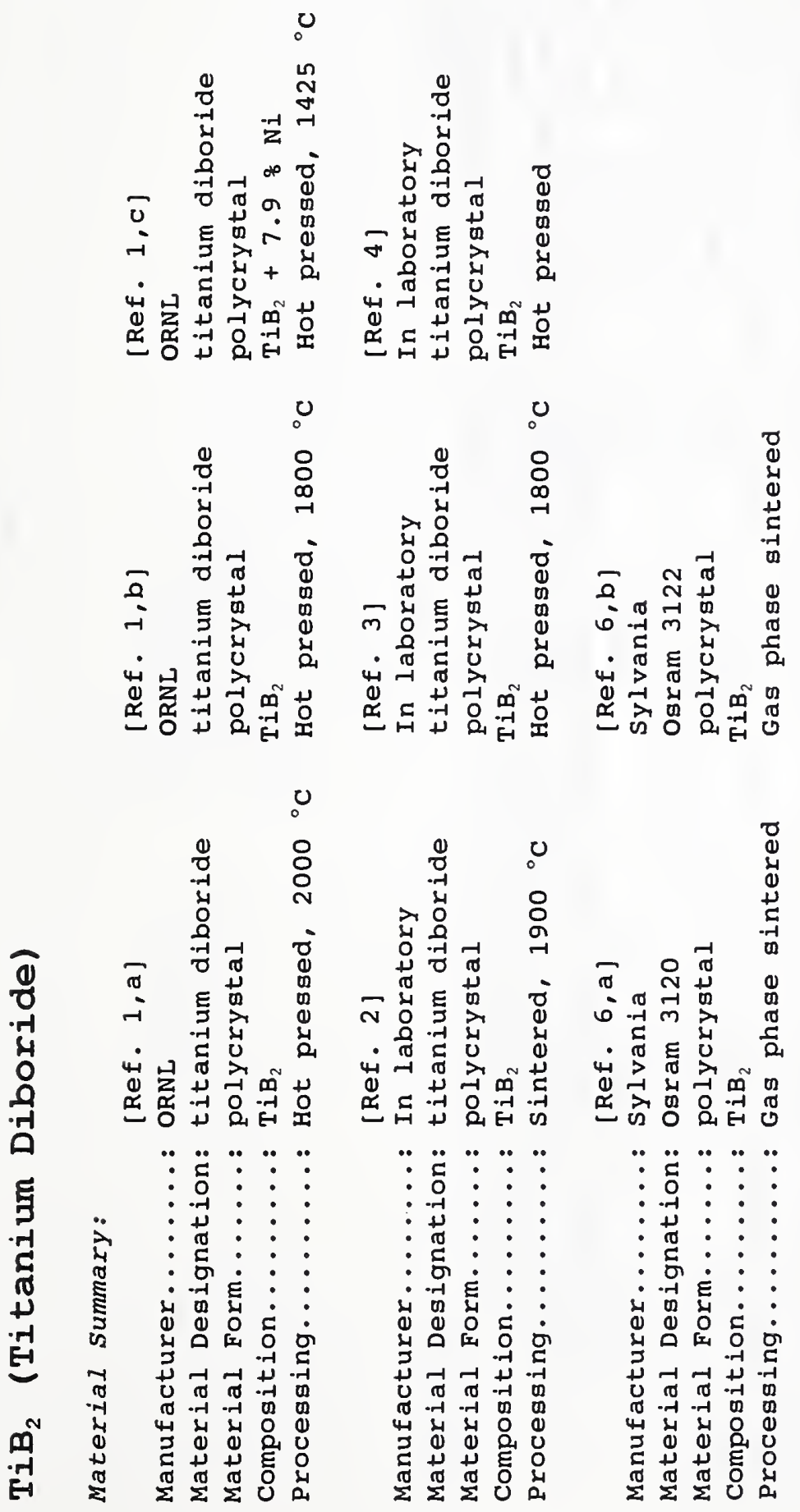

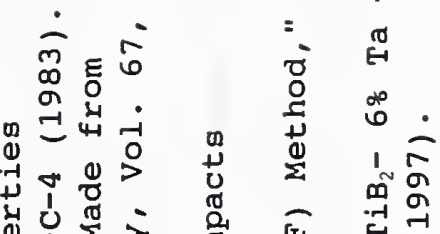

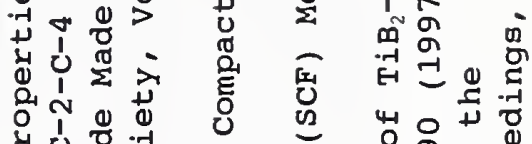
क्ष.

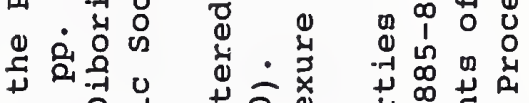

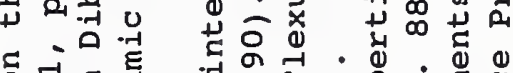
ठี ये उ

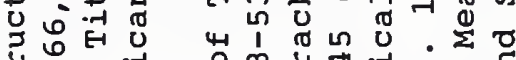

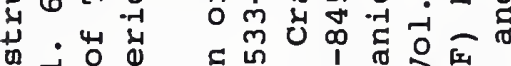

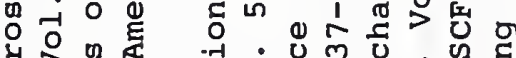

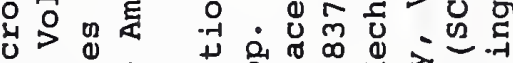

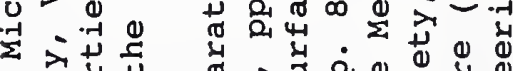

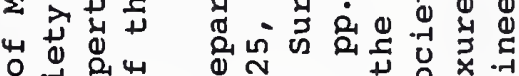

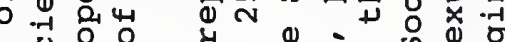
艺苍员 出 0 己 出者

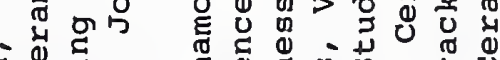
造芯 =

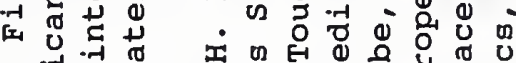

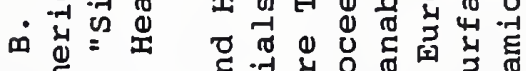
政0 ब

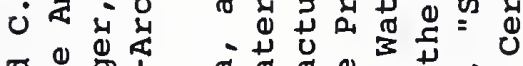

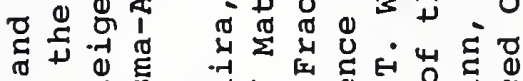

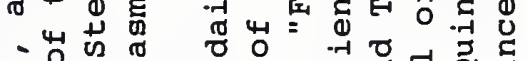

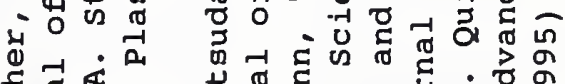
ป

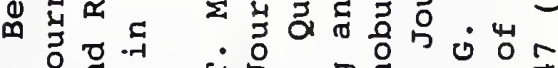

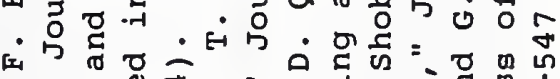

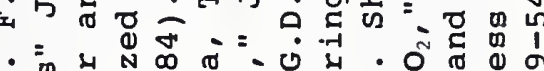

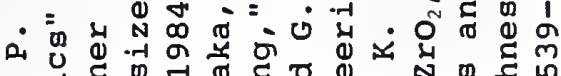

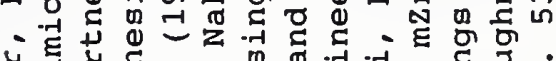

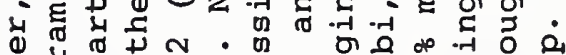

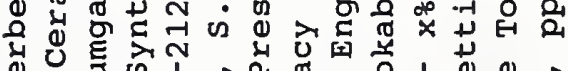

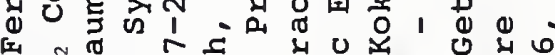
๓

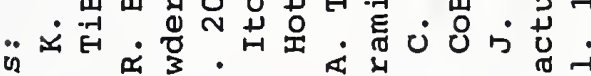

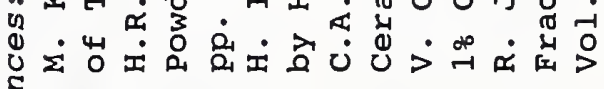
离 


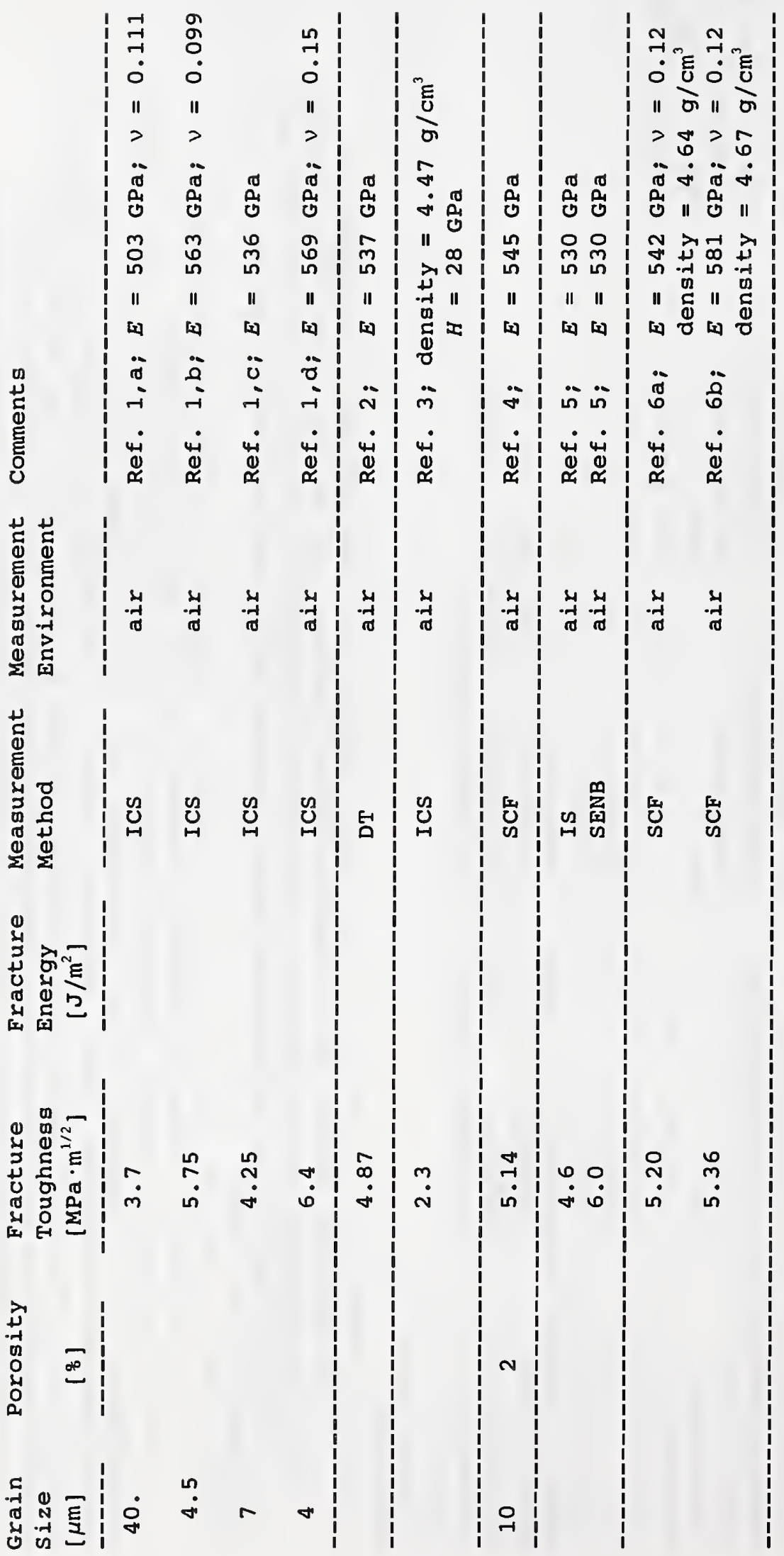




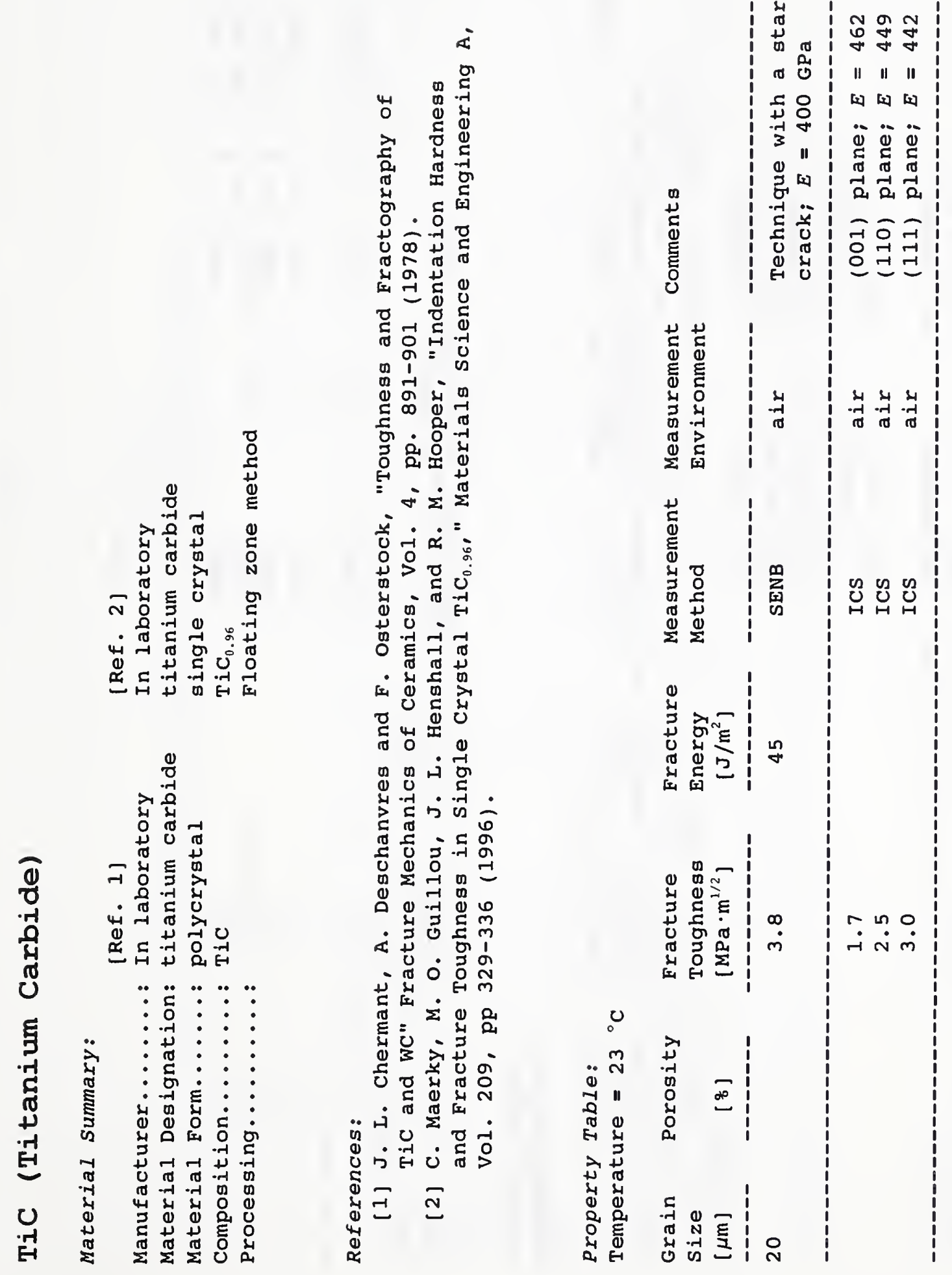




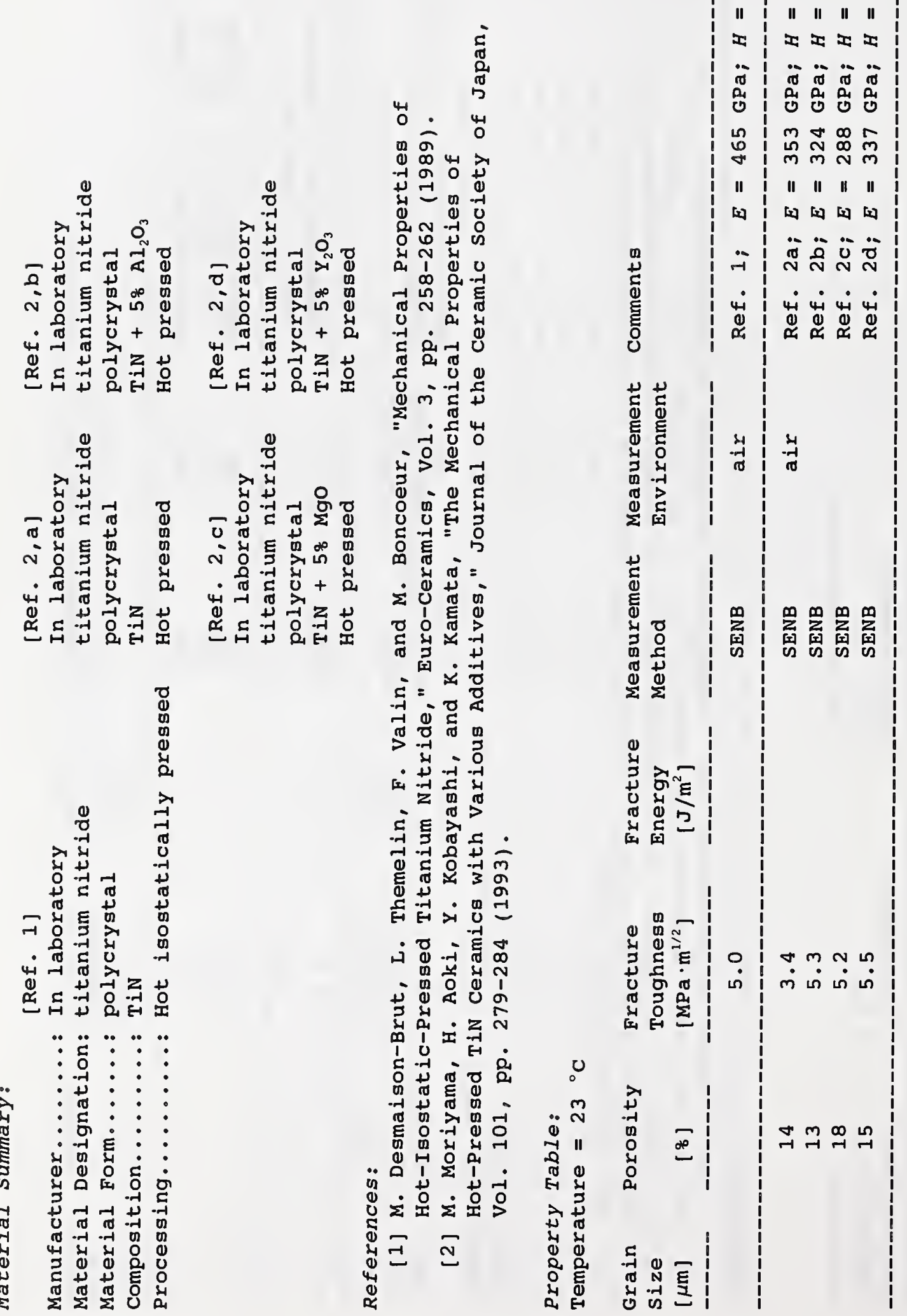

$\stackrel{1}{2}$ 


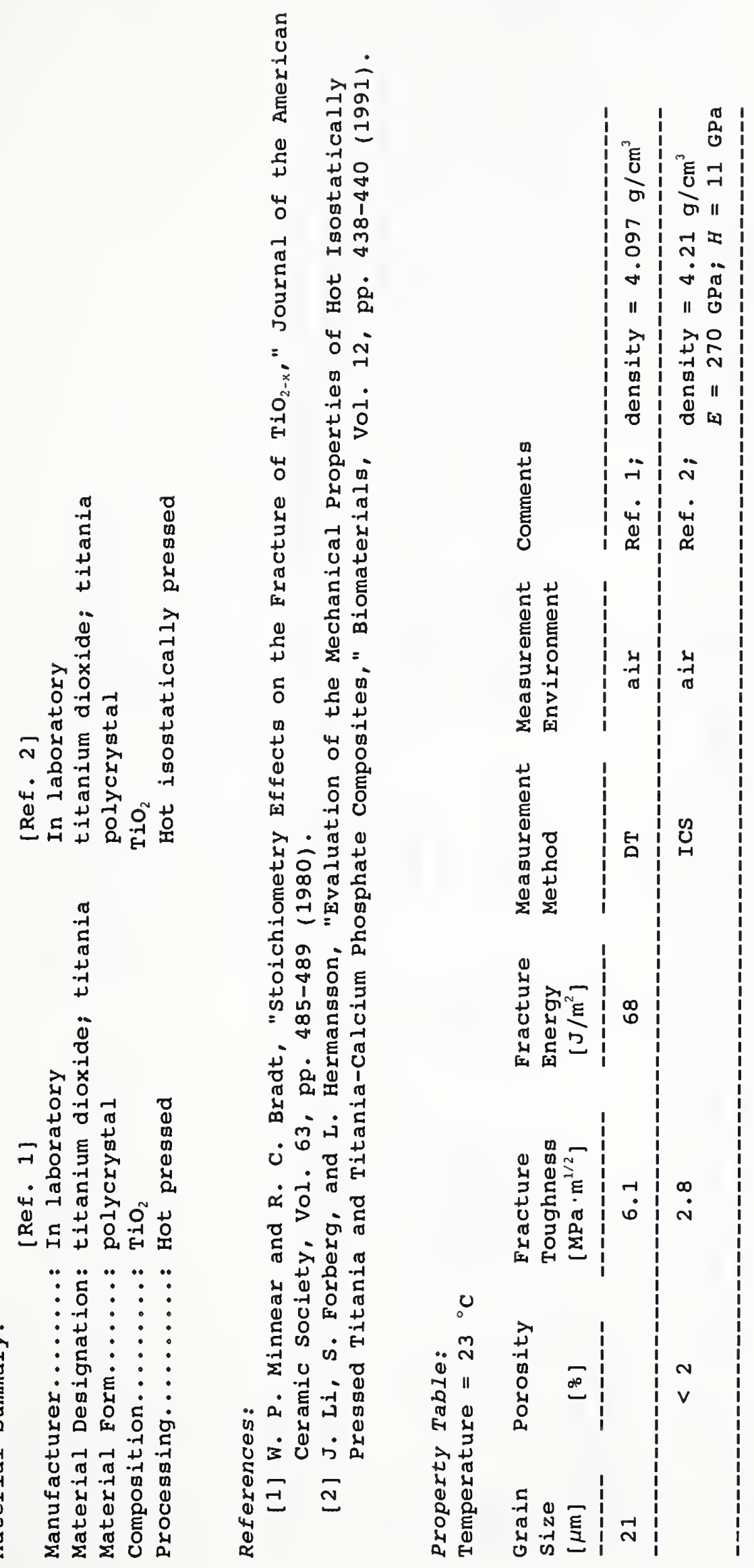




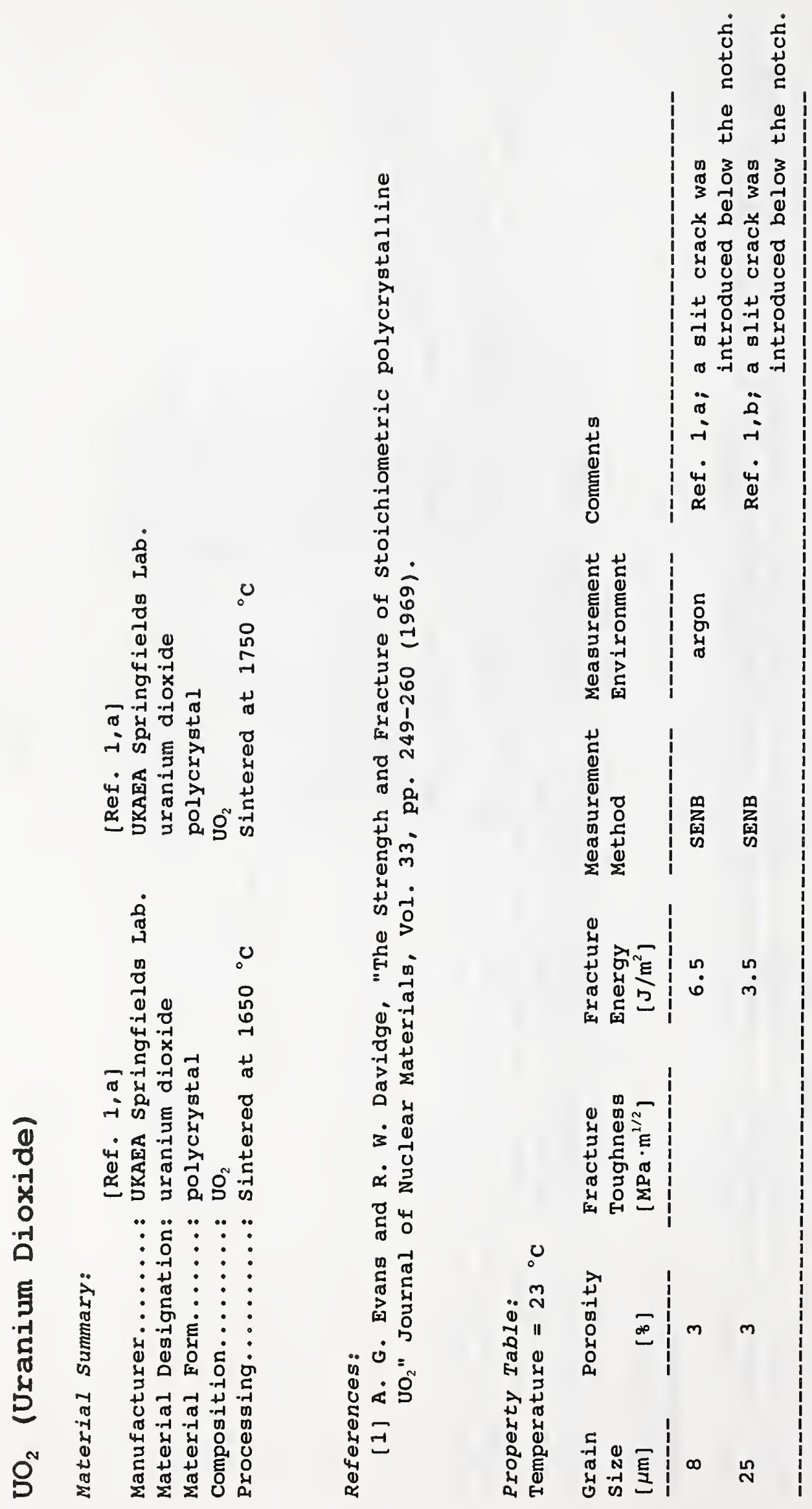



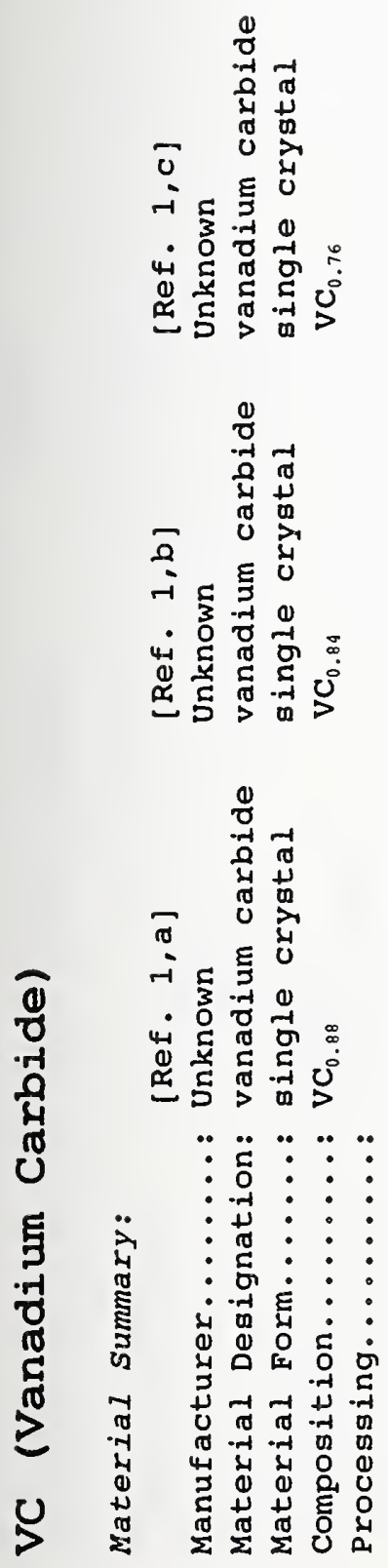

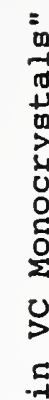

.

品交

ป

亩 㶽

๗.

证

舤

ธ。

in

:

宾

०

造

ㅇํㅇ

苟

की

농

7

${ }_{-1}^{-1}$

o

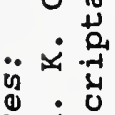

ठैं

ฮี

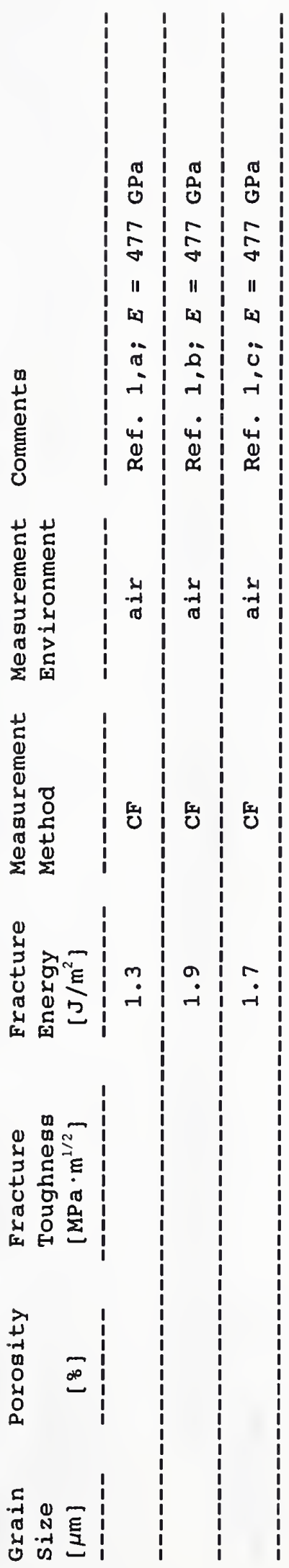

$\frac{3}{1}$ 

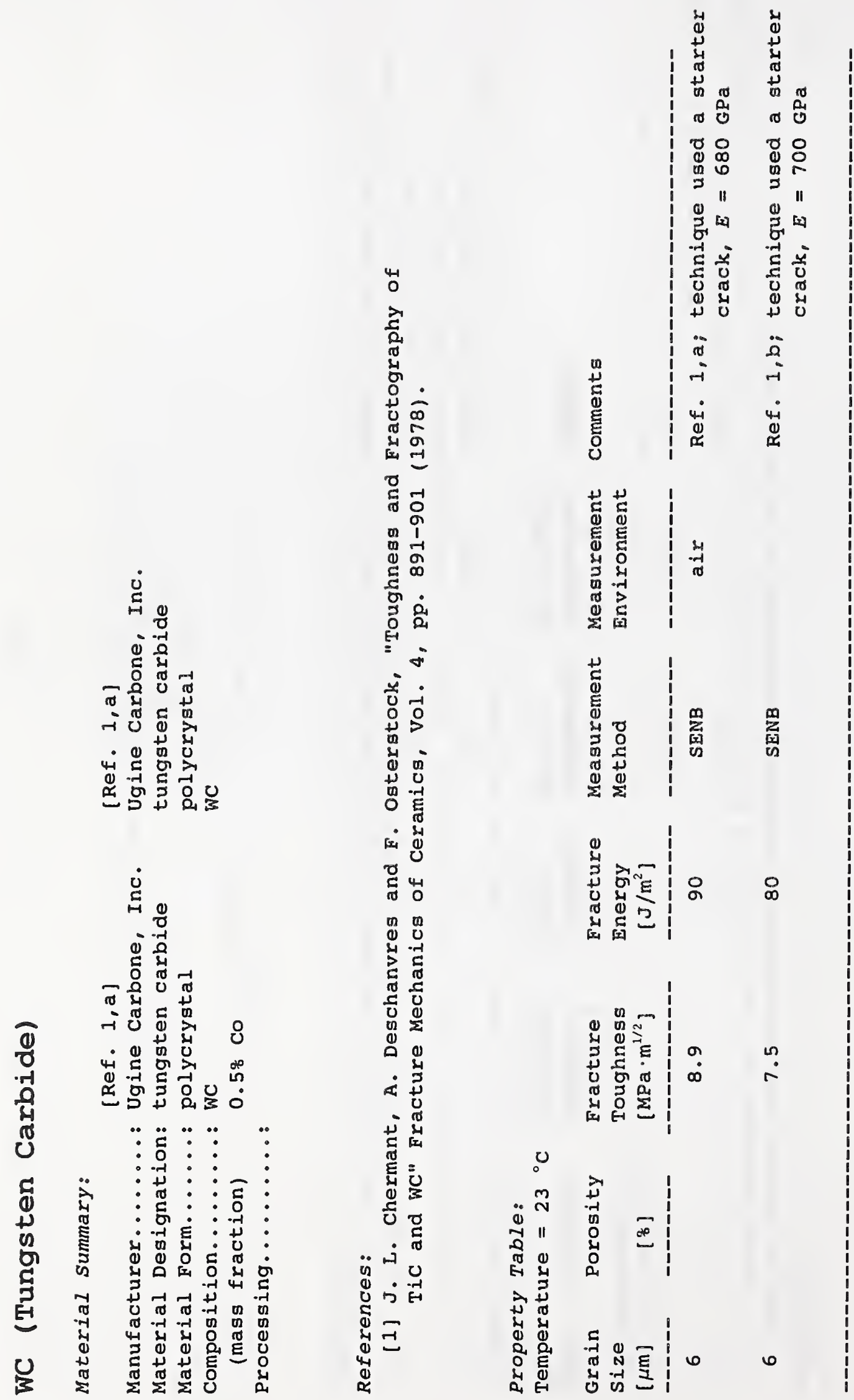


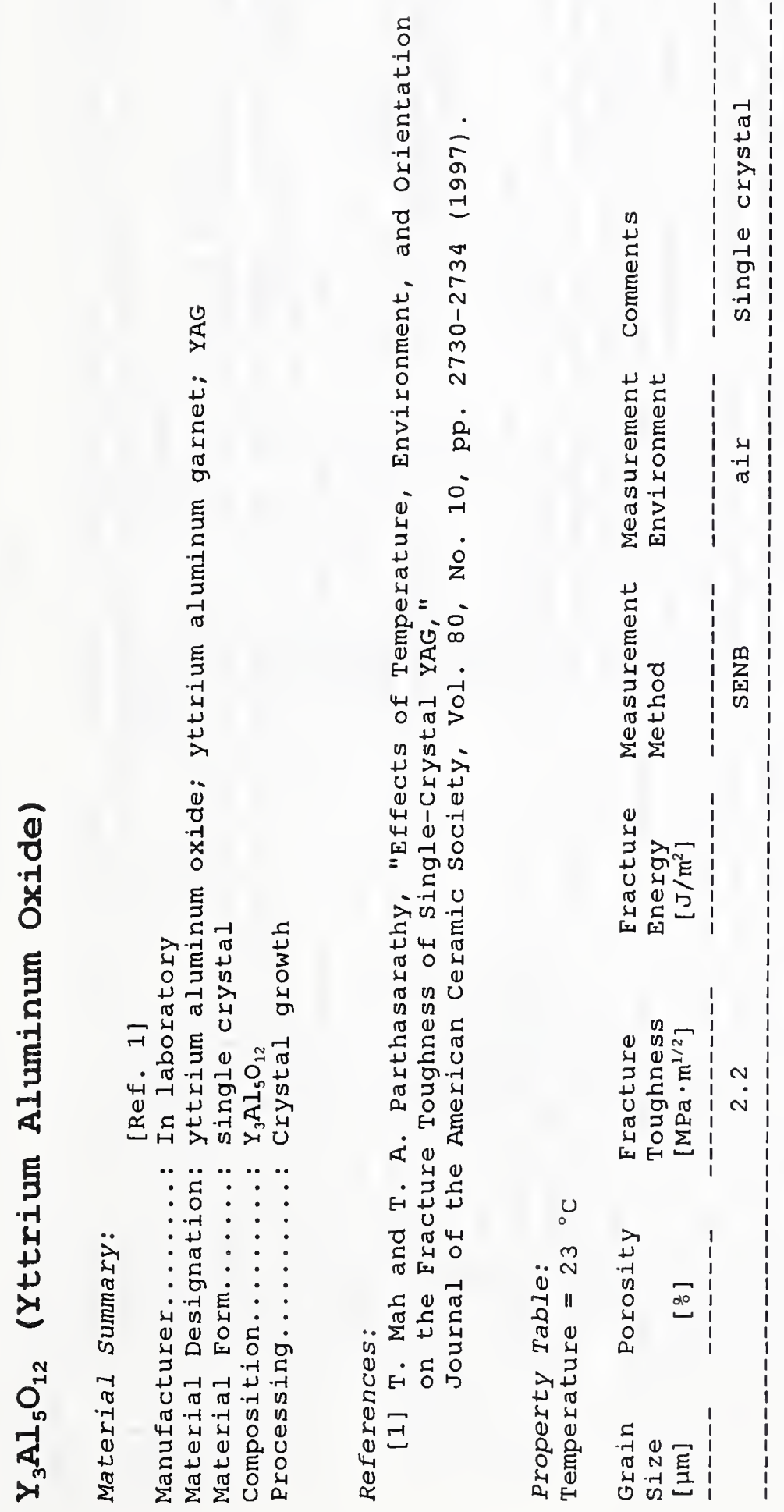




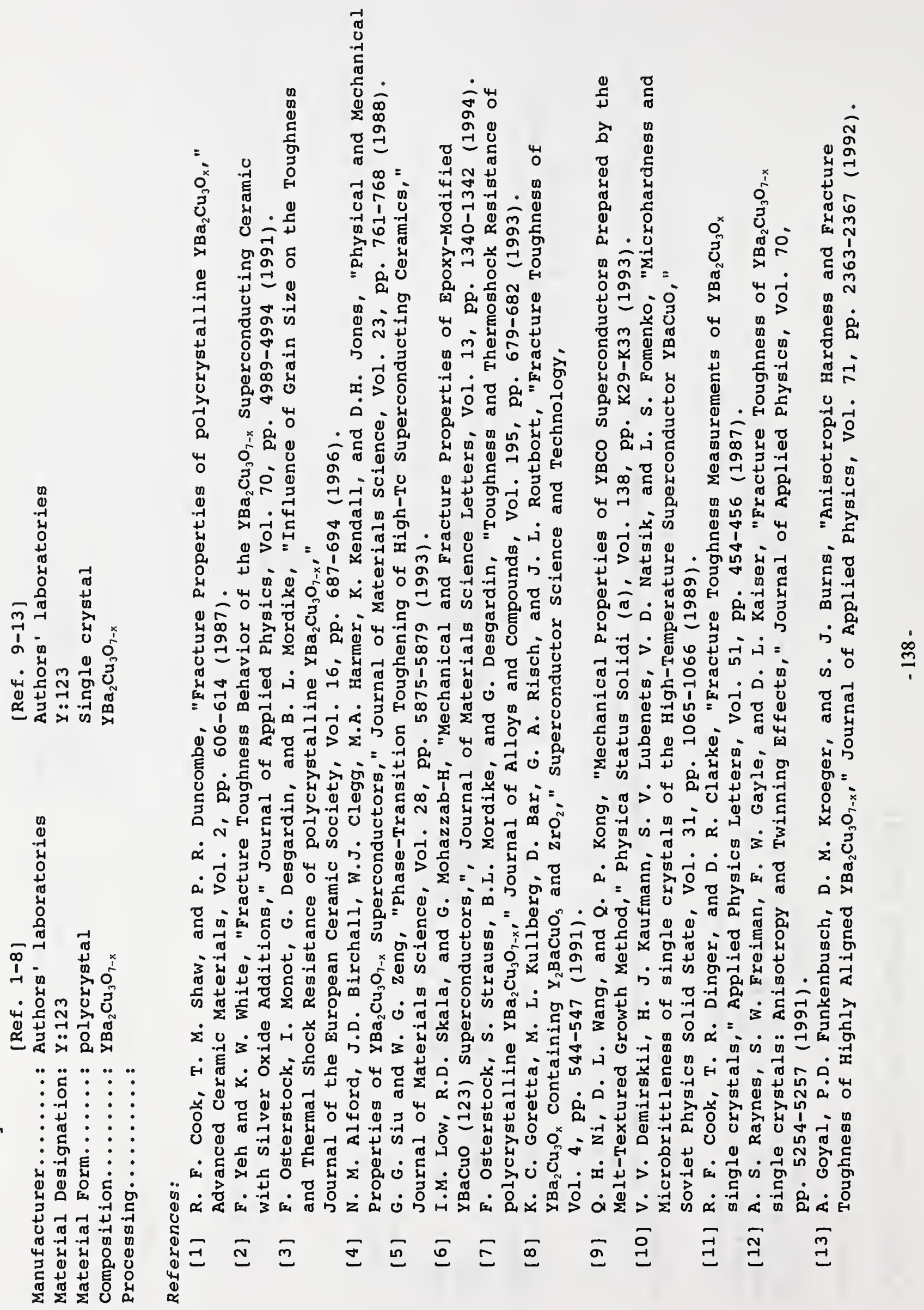




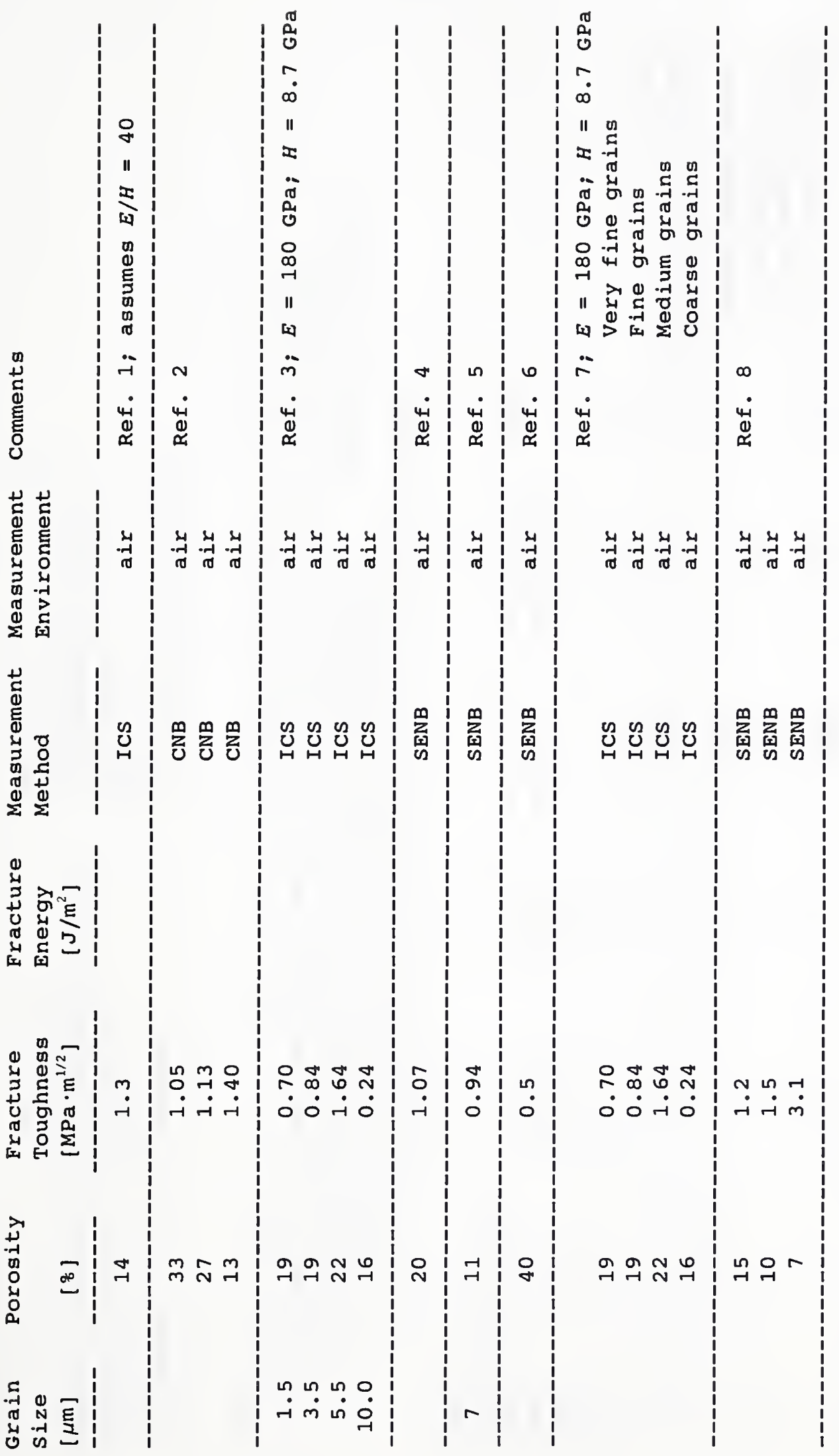




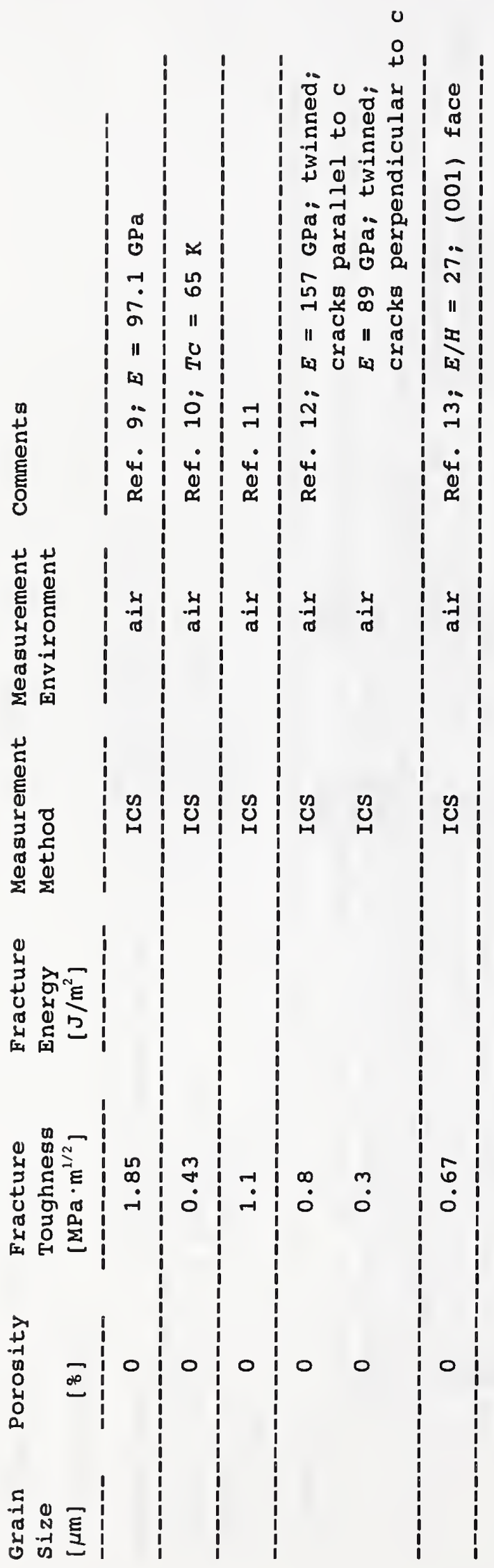

亨 


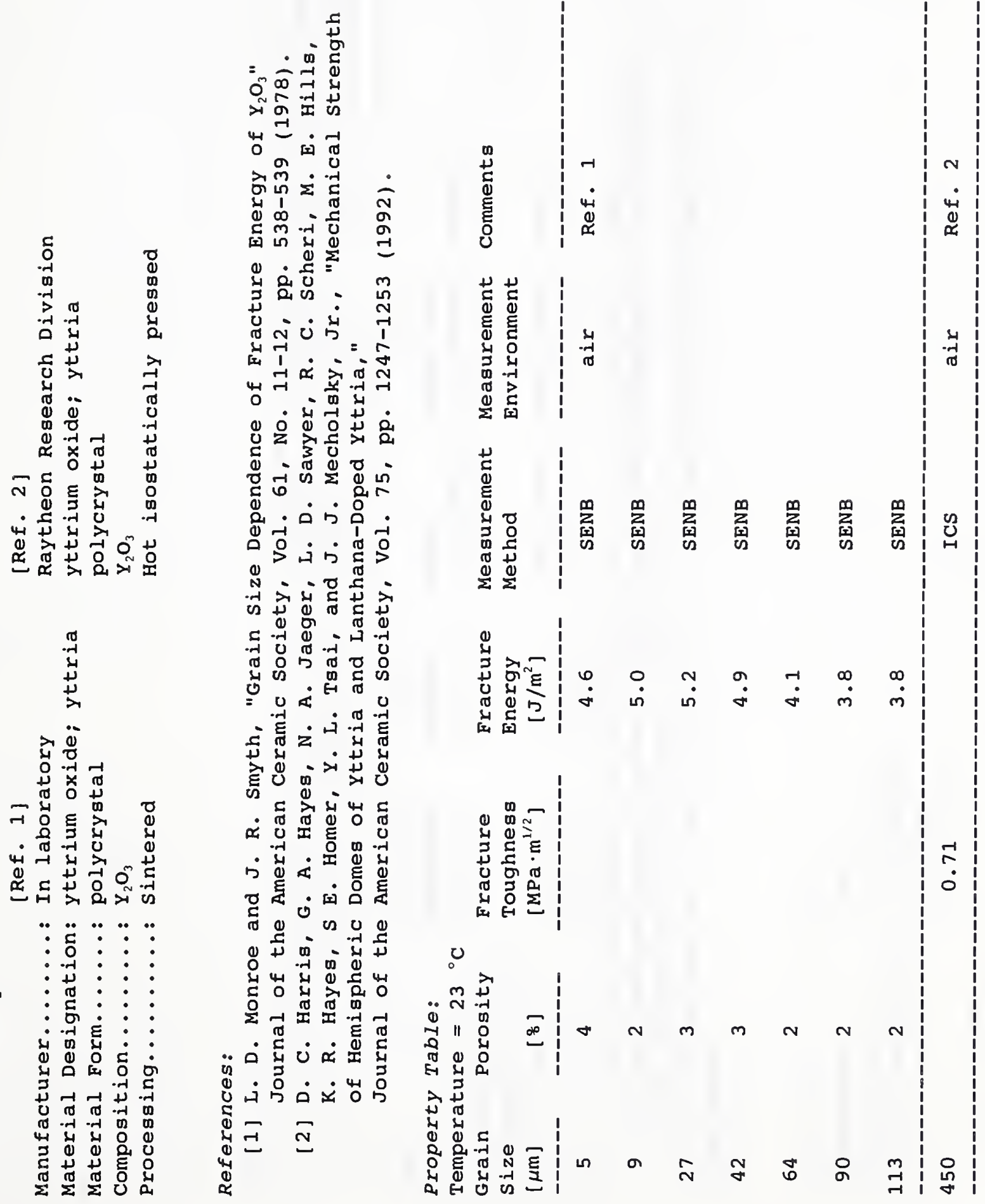



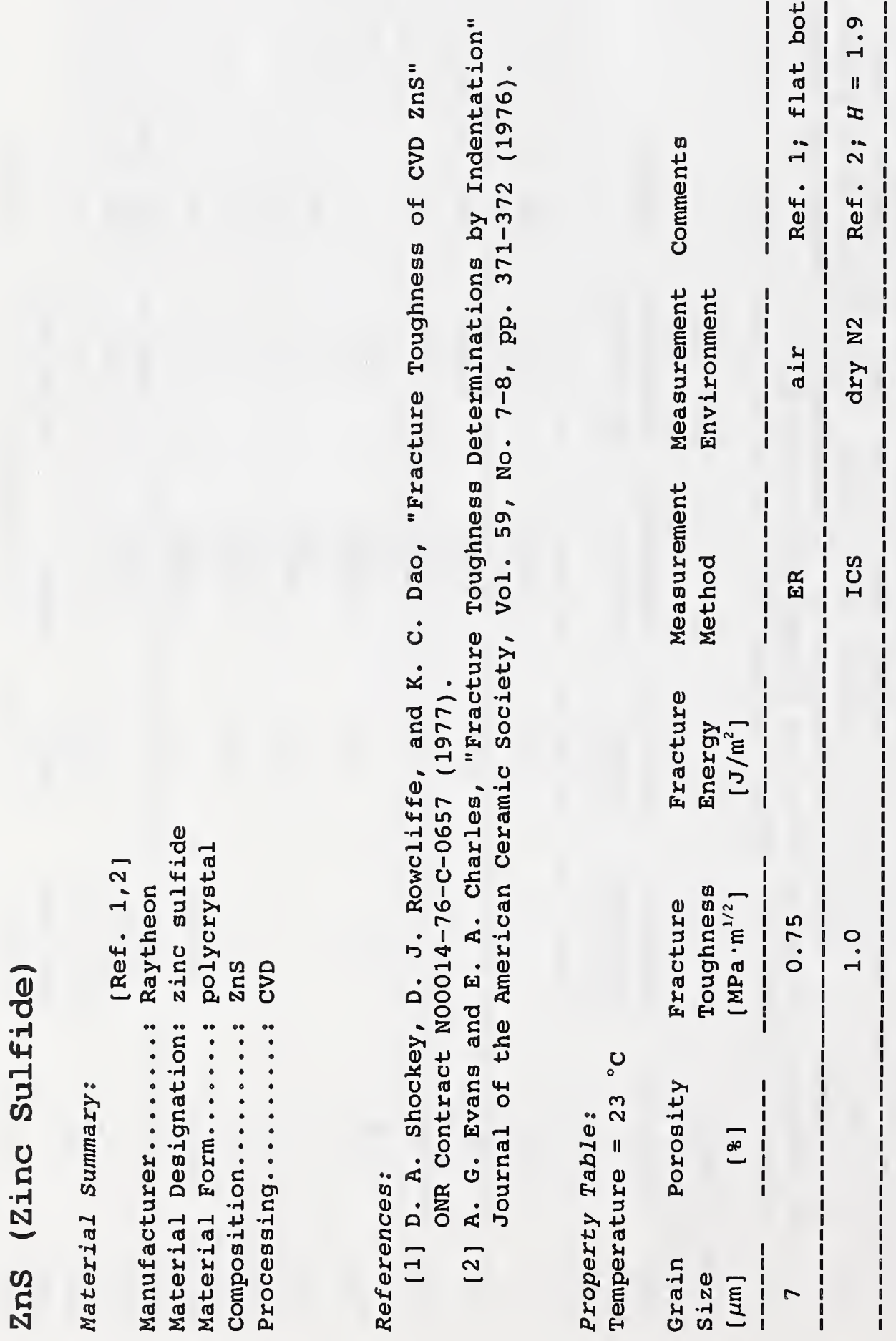


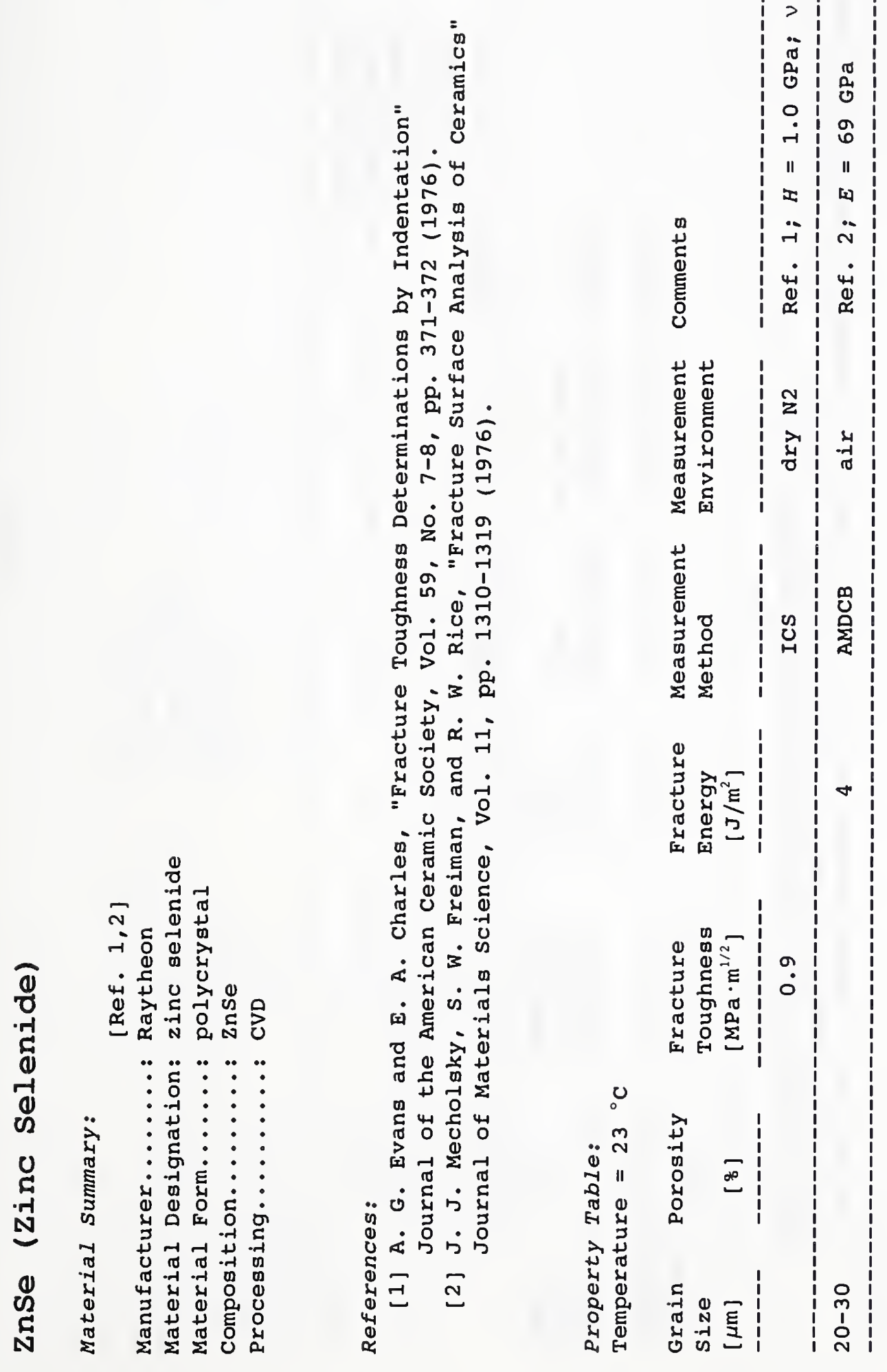




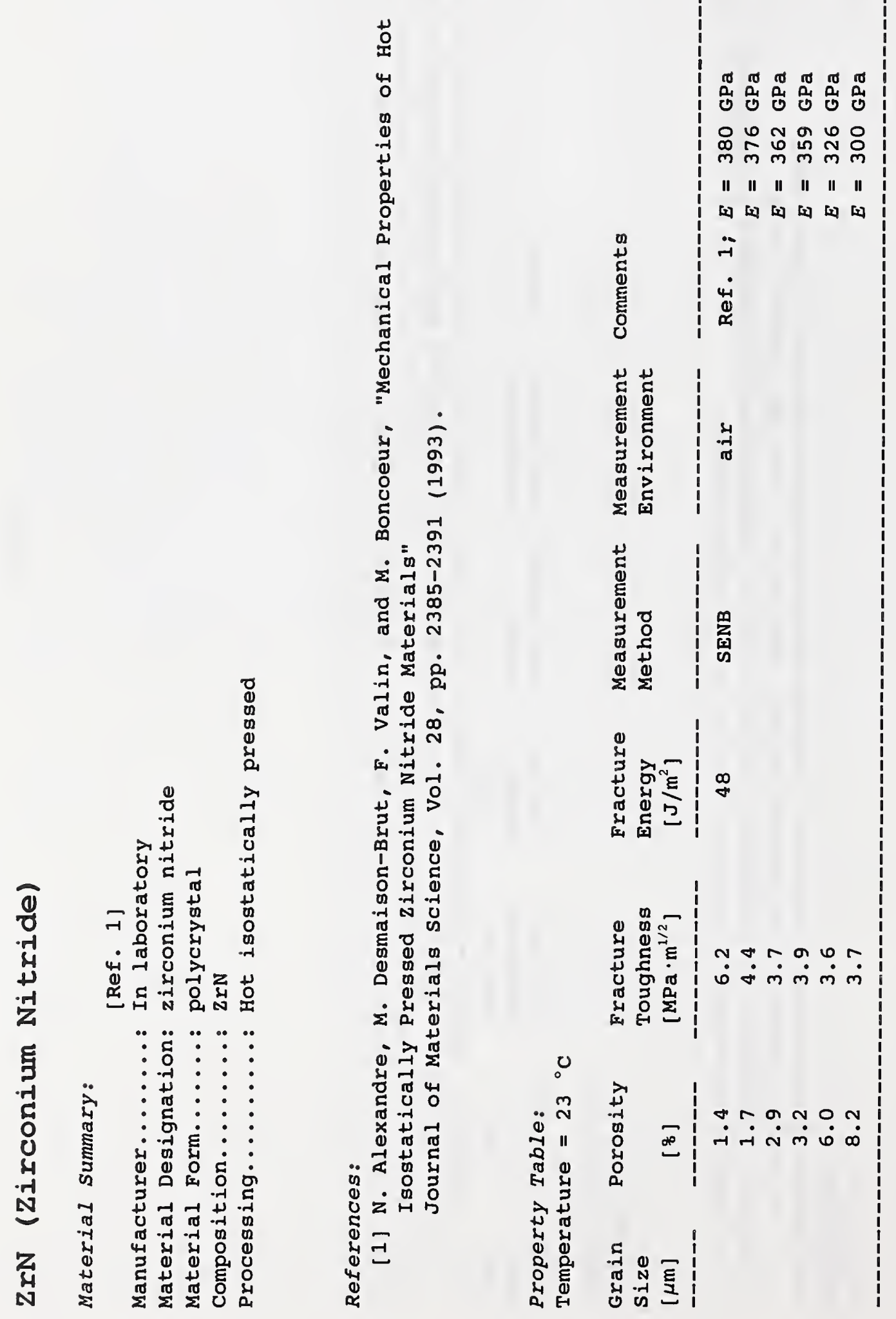




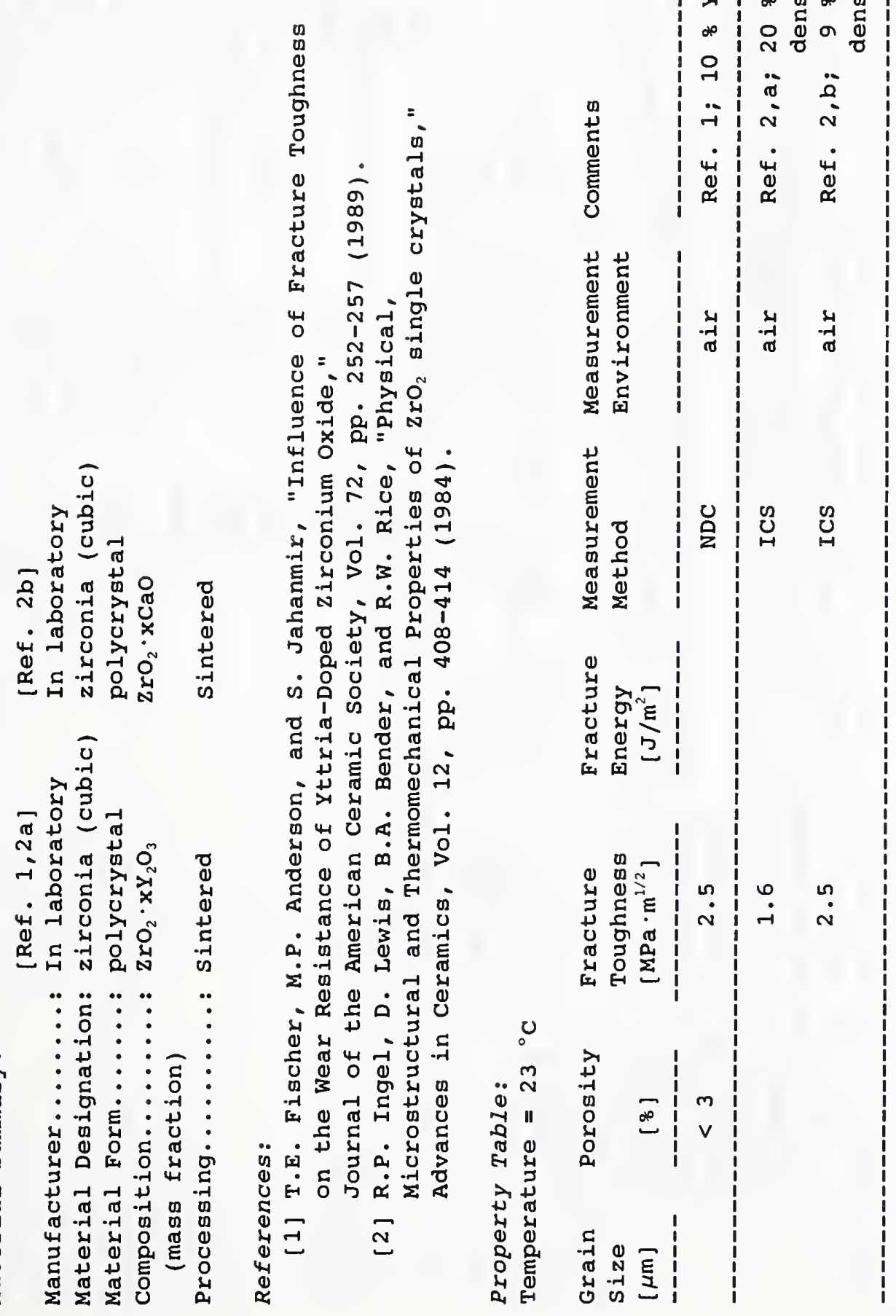




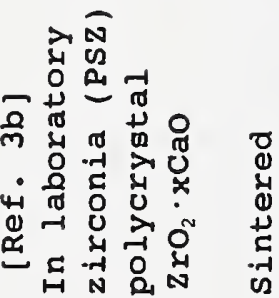

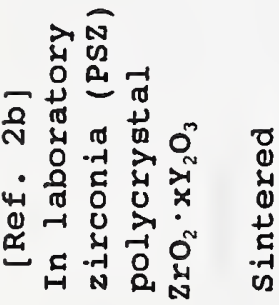

त)

व. क्ष

亦蒈

N

ณ

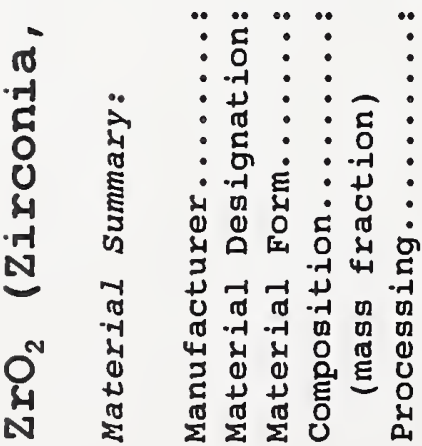

- $\begin{array}{r}4 \\ 0\end{array}$

๙ิ

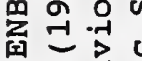

đี

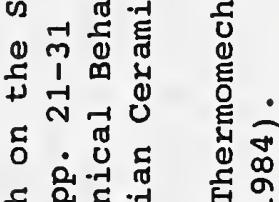

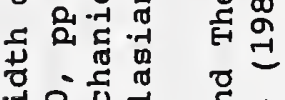

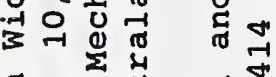

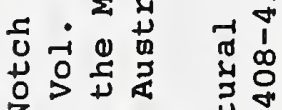

㟧

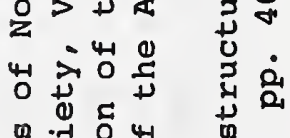

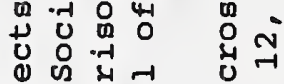

出 0 要

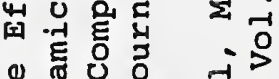

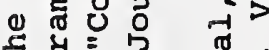

至造=

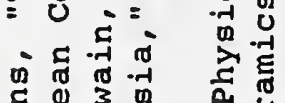

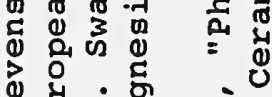

过

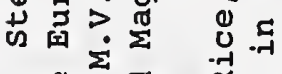

㝳

荧㟧

至望势

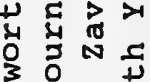

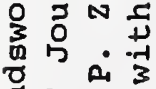

$\pi=\Delta \frac{1}{3}$

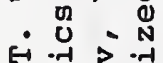

垔

है

द颔

से

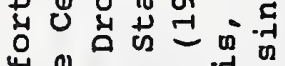

岂。 0

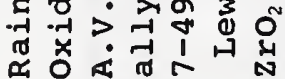

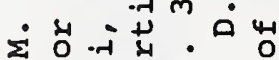

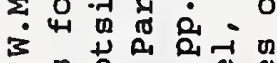

-

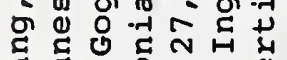

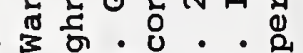

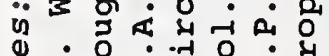

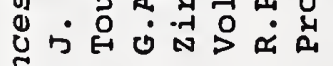

离

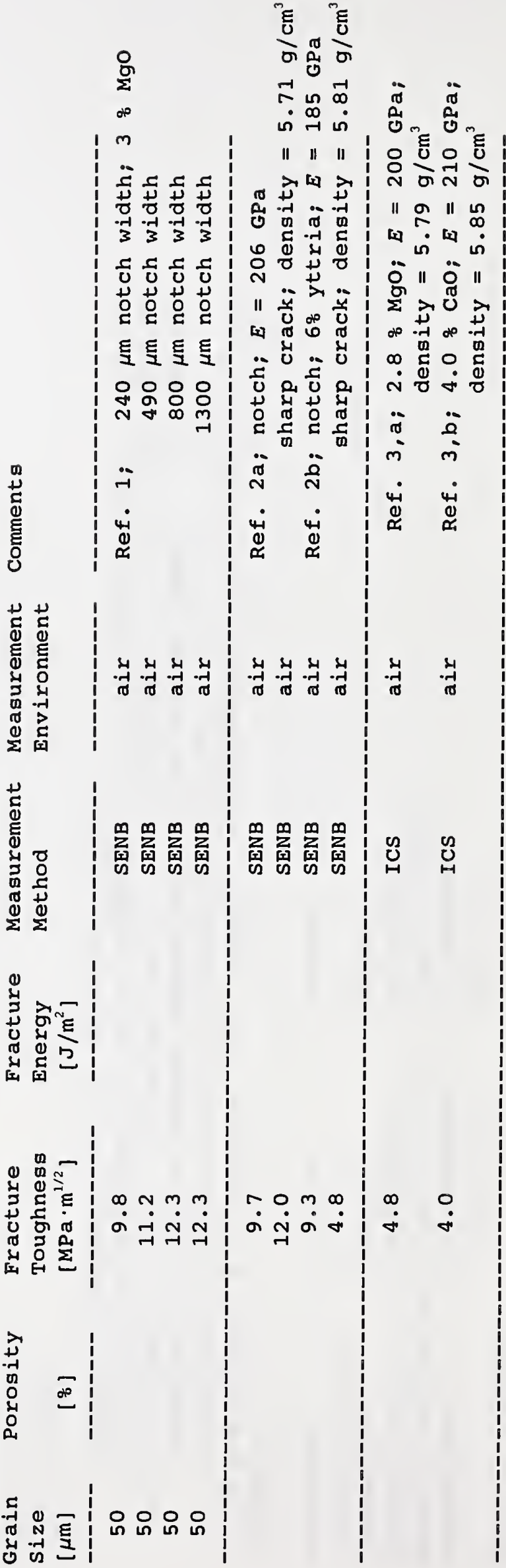

to 

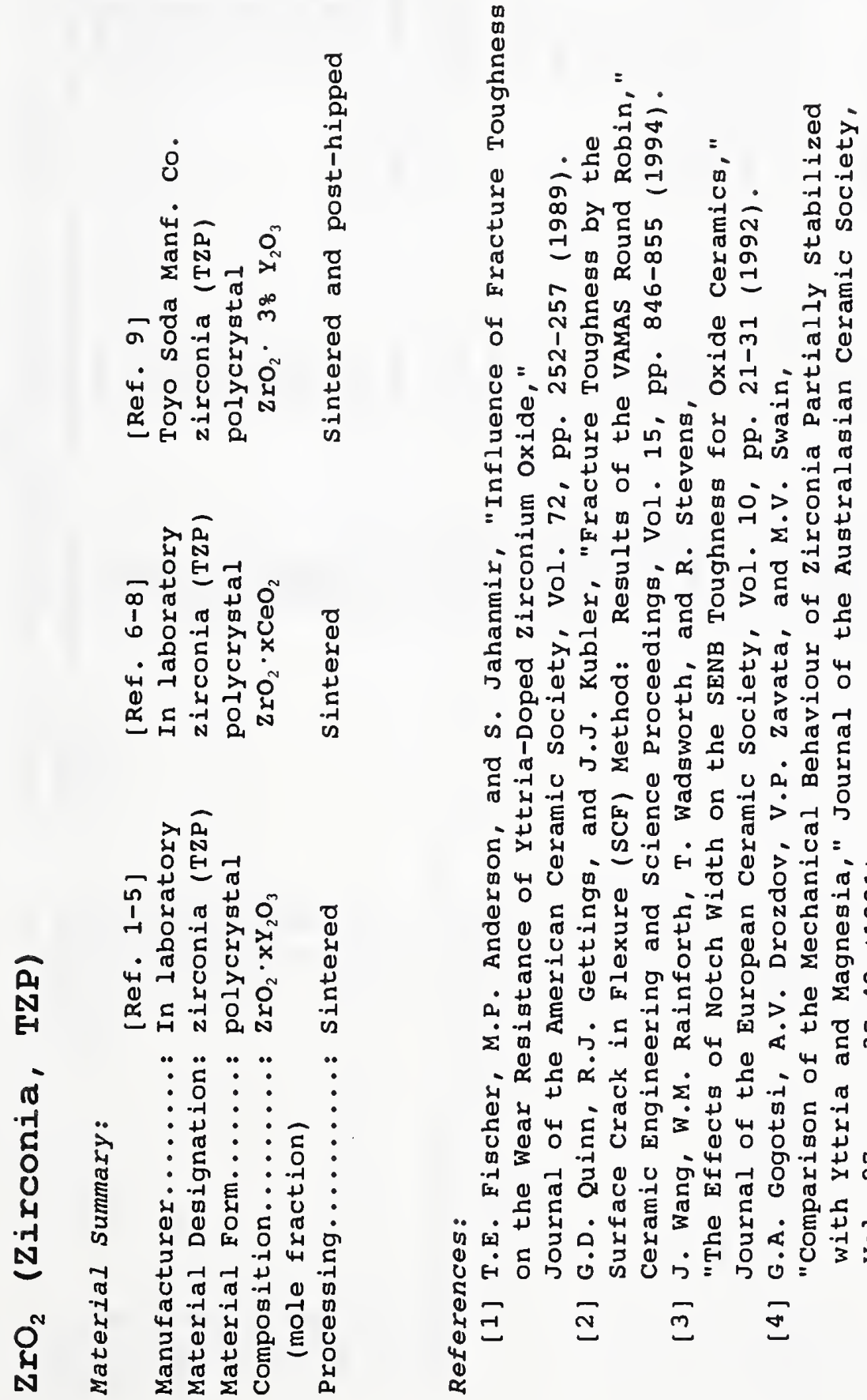

=

خ्रे.

要完

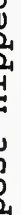

ซ్ర

ס

㟧

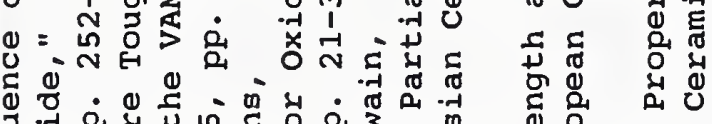

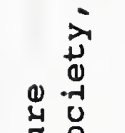

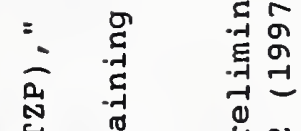

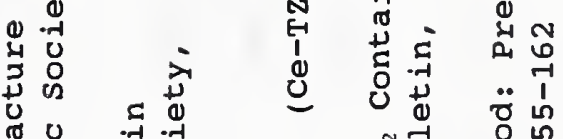

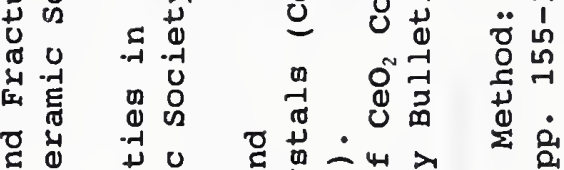

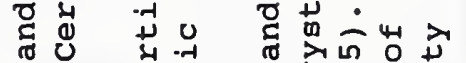

岂

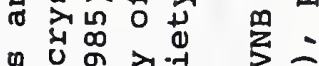

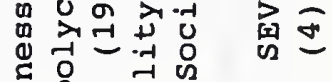

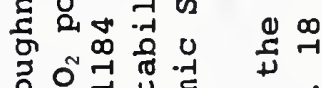

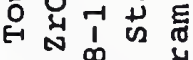

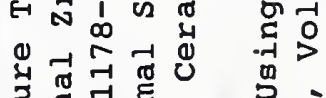

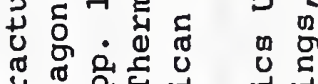
出

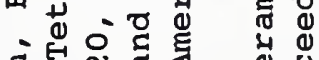

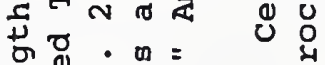
कृ

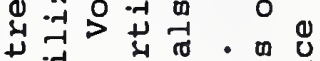

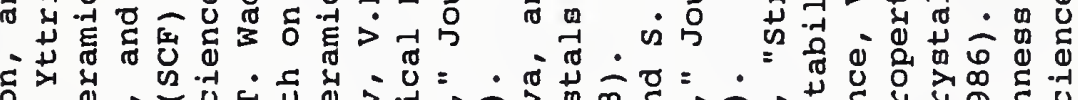

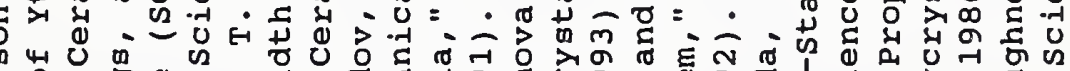

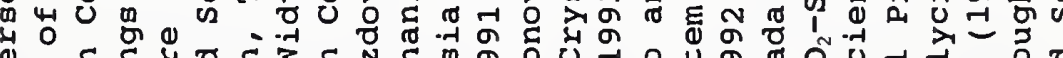
0

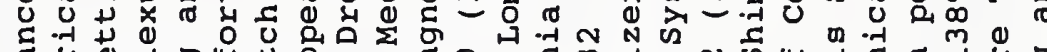
. की

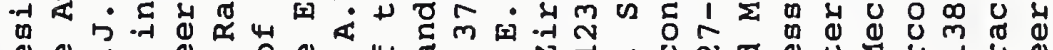

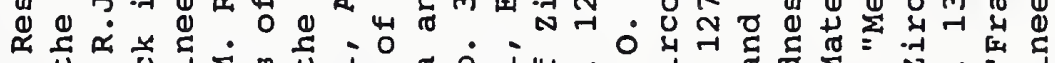

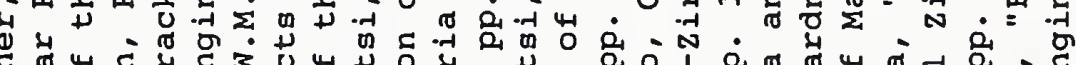

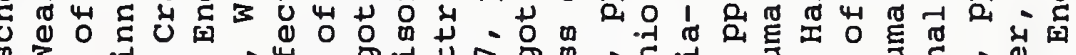
-

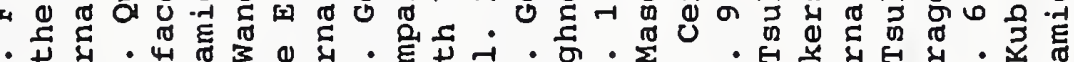

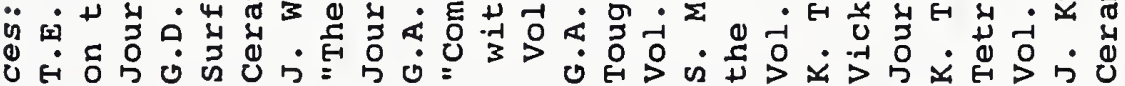

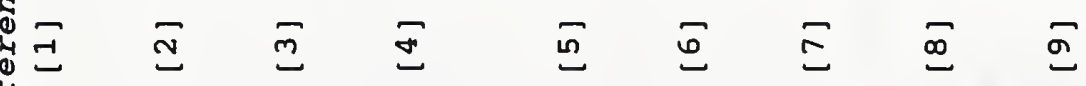




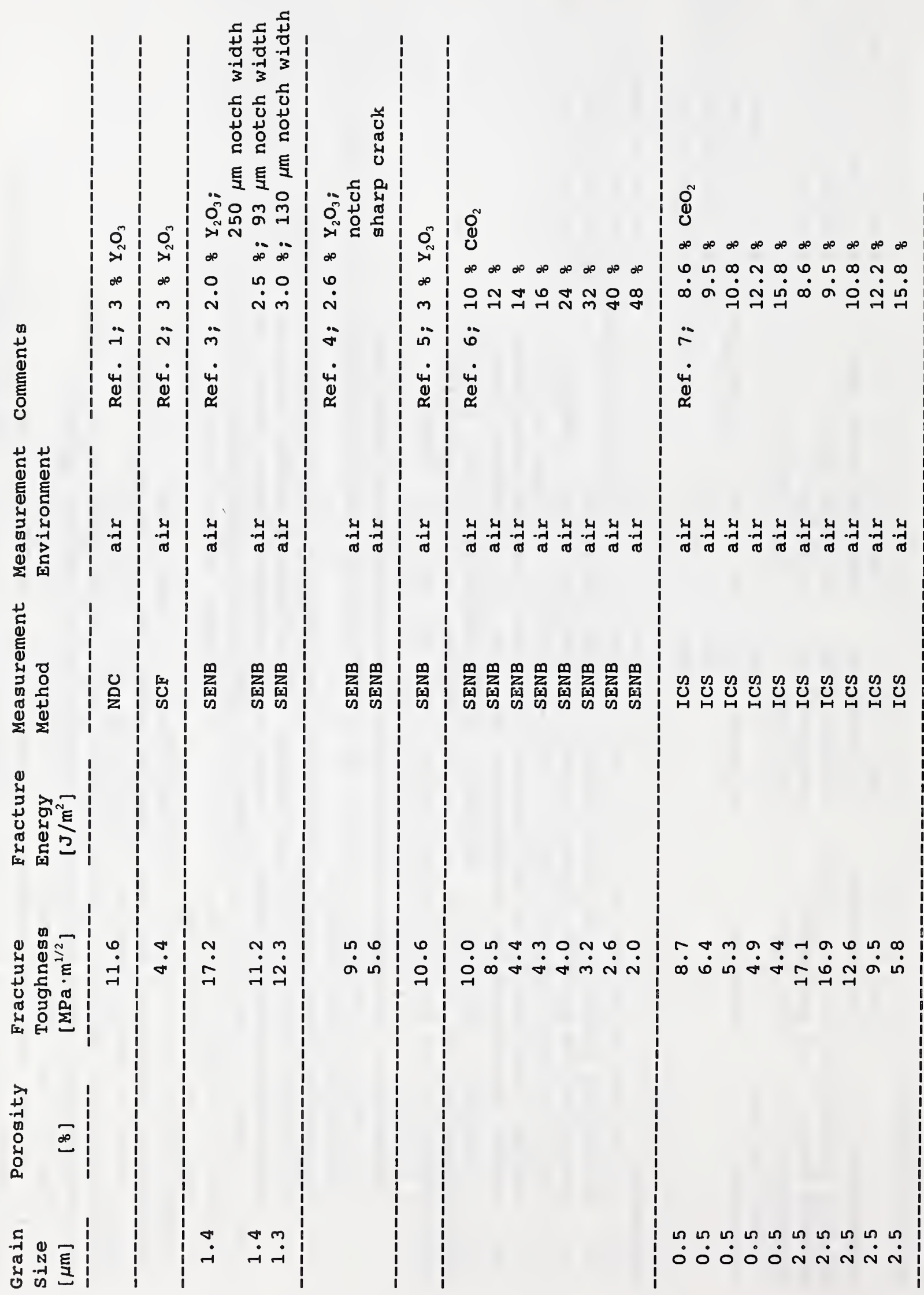




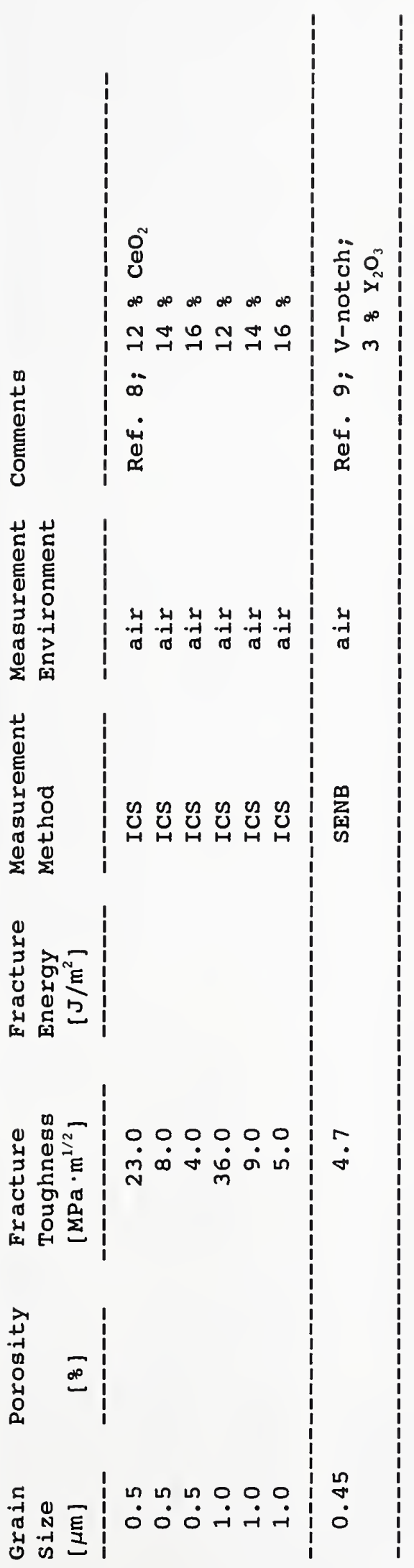




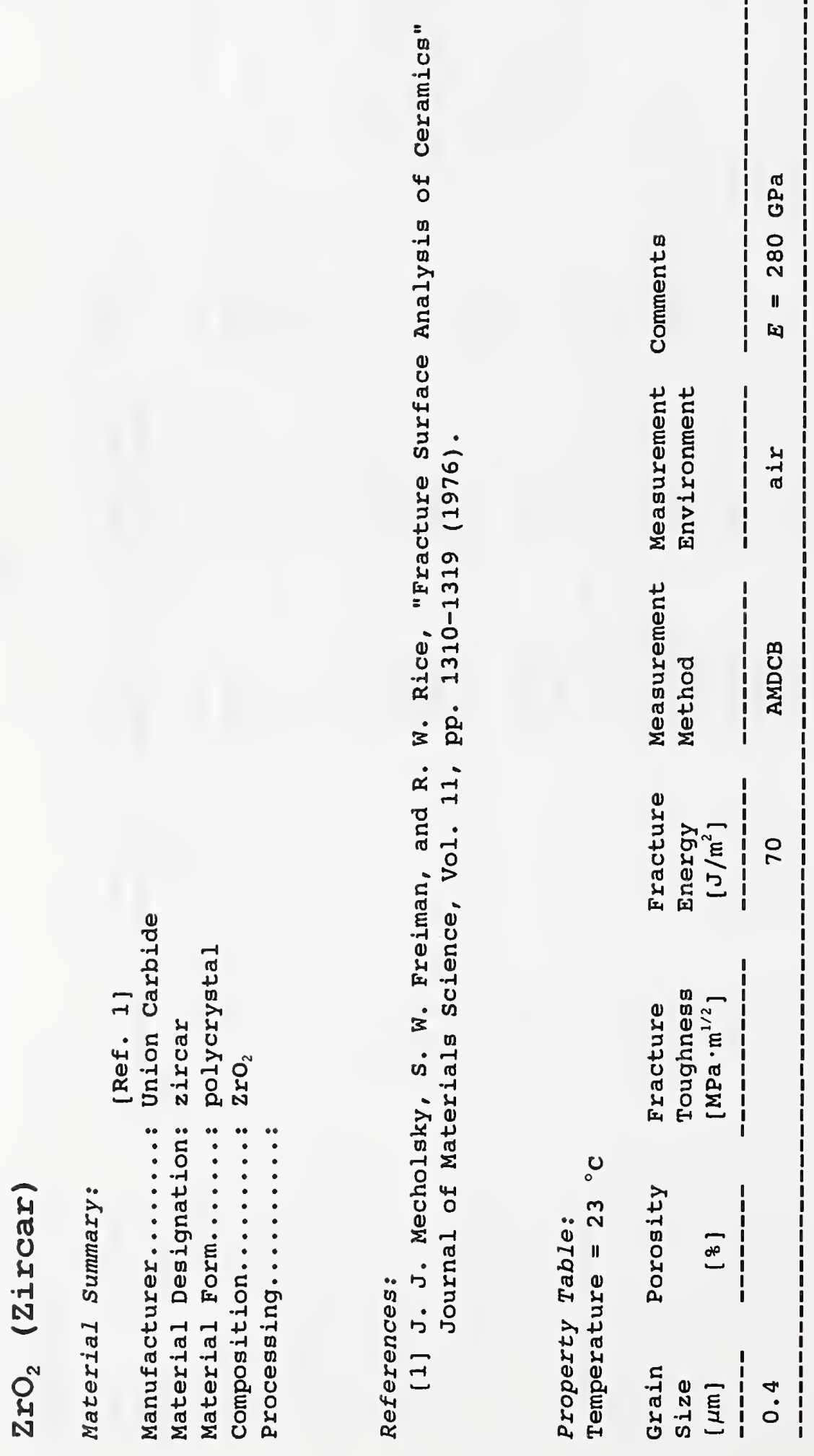




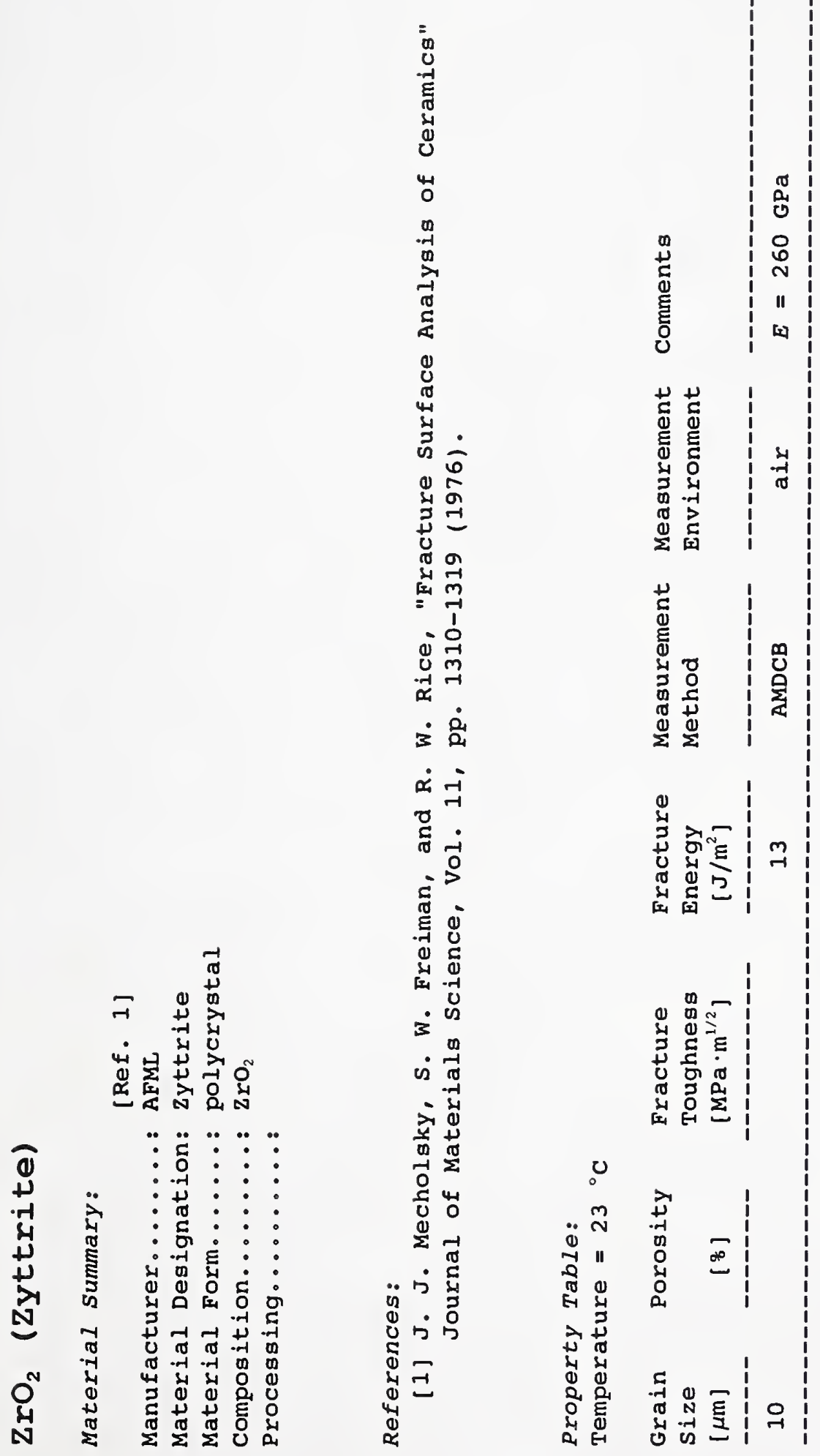



